T. Kimura

Nagoya Math. J.

Vol. 85 (1982), 1-80

\title{
THE $b$-FUNCTIONS AND HOLONOMY DIAGRAMS OF IRREDUCIBLE REGULAR PREHOMOGENEOUS VECTOR SPACES
}

\author{
TATSUO KIMURA
}

\section{Introduction}

The purpose of this paper is to investigate the micro-local structure and to calculate, by constructing the holonomy diagrams, the $b$-functions (See [2]) of irreducible regular prehomogeneous vector spaces (See [1]).

Since we know the relation of $b$-functions with respect to castling transformations (See $\S 12$ ), it is enough to calculate them only when they are reduced. In this paper, we shall deal with twenty of all twenty nine reduced regular P.V.'s in the Table in [1]. Together with other articles, this completes the list of $b$-functions of irreducible reduced regular prehomogeneous vector spaces (See $\S 12)$ except $\left(S L(5) \times G L(4), \Lambda_{2} \otimes \Lambda_{1}, V(10)\right.$ $\otimes V(4)$ ) which is the most complicated case (See I. Ozeki [11]). This paper consists of the following twelve sections and one Appendix with I. Ozeki.

$\S$ 1. Preliminaries

$\S$ 2. Regular P.V.'s related with $G L(n)$

$\S$ 3. $\left(S p(n) \times G L(2 m), \Lambda_{1} \otimes \Lambda_{1}, V(2 n) \otimes V(2 m)\right)$

$\S$ 4. (Spin $(10) \times G L(2)$, half-spin rep. $\left.\otimes \Lambda_{1}, V(16) \otimes V(2)\right)$

$\S$ 5. $(G L(1) \times \operatorname{Spin}(12), \square \otimes$ half-spin rep., $\quad V(1) \otimes V(32))$

$\S$ 6. $\left(G L(1) \times E_{6}, \square \otimes \Lambda_{1}, V(1) \otimes V(27)\right)$

$\S$ 7. $\left(G L(1) \times E_{7}, \square \otimes \Lambda_{6}, V(1) \otimes V(56)\right)$

$\S$ 8. $\left(G L(6), \Lambda_{3}, V(20)\right)$

$\S$ 9. $\left(G L(1) \times S p(3), \square \otimes \Lambda_{1}, V(1) \otimes V(14)\right)$

$\S 10 . \quad\left(G L(7), \Lambda_{3}, V(35)\right)$

$\S 11 . \quad\left(S L(5) \times G L(3), \Lambda_{2} \otimes \Lambda_{1}, V(10) \otimes V(3)\right)$

$\S 12$. Table of the $b$-bunctions of irreducible reduced regular P.V.'s

Received January 11, 1979

Revised September 7, 1979 
Appendix with I. Ozeki. $(G L(1) \times \operatorname{Spin}(14), \square \otimes$ half-spin rep., $V(1)$ $\otimes V(64))$

In $\S 1$, we shall review the main results of [2] which will be used later. From $\S 2$ to $\S 11$, we do the classification of the orbits, construction of the holonomy diagrams and calculation of the $b$-functions. In $\S 12$, we shall give the list of $b$-functions for irreducible reduced regular P.V.'s. Some of them have been already calculated by M. Sato and the author using the different method (See [7]). The holonomy diagrams in $\S 2, \S 8$ and $\S 10$ are first obtained by M. Sato. The author would like to express his hearty thanks to Professors Mikio Sato and Masaki Kashiwara for their invaluable advice and encouragement.

\section{§1. Preliminaries}

Let $(G, \rho, V)$ be an irreducible regular prehomogeneous vector space (abbrev. P.V.) with the singular set $S$. Then $S$ is the zeros of the relative invariant $f(x): S=\{x \in V ; f(x)=0\}, f(\rho(g) x)=\chi(g) f(x)$ for all $g \in G$ and $x \in V$. We shall consider the micro-differential equations $\mathfrak{M}=\mathscr{E} f^{s}$ where $\mathscr{E}$ is the sheaf of micro-differential operators of finite order on the cotangent bundle $T^{*} V=V \times V^{*}$ (See [2]). Note that the group $G$ acts on $T^{*} V$ by $(x, y) \mapsto\left(\rho(g) x, \rho^{*}(g) y\right)$ for $x \in V, y \in V^{*}$ and $g \in G$ where $\rho^{*}$ denotes the contragredient representation of $\rho$. Let $\Lambda$ be the Zariski-closure of a conormal bundle of some $G$-orbit $\rho(G) \cdot x_{0}\left(x_{0} \in V\right)$. Since we consider only the Zariski-closure of a conormal bundle, we shall omit the word "the Zariski-closure" for simplicity. Assume that $\Lambda$ is $G$-prehomogeneous and is contained in $W=\overline{\left\{\left(x, \operatorname{grad} \log f(x)^{s}\right) ; x \in V-S, s \in C\right\}}$. In this case, $A$ is called a good holonomic variety. It is an irreducible component of the characteristic variety of $\mathfrak{M}$. We can show that there exists a local $b$ function $b_{A}(s)$ which is unique up to a constant multiple (See [2]). We have $b_{V \times\{0\}}(s)=1$ and $b_{\{0\} \times V^{*}}(s)=b(s)$ where $b(s)$ denotes the $b$-function of this P.V. When two good holonomic varieties $\Lambda_{0}$ and $\Lambda_{1}$ intersect with codimension one, we have the relation between $b_{\Lambda_{0}}(s)$ and $b_{A_{1}}(s)$ as follows (See [2]).

Theorem 1-1 ([2] Theorem 7-5). Let $\Lambda_{0}$ and $\Lambda_{1}$ be good holonomic varieties whose intersection is of codimension one with the intersection exponent $(\mu: \nu)$. Assume that $\mathfrak{M}=\mathscr{E} f^{s}$ is a simple holonomic system with support $\Lambda_{0} \cup \Lambda_{1}$ and $\Lambda_{0} \cap \Lambda_{1} \not \subset \overline{\text { supp } M-\left(\Lambda_{0} \cup \Lambda_{1}\right)}$. Assume that $m_{0}>m_{1}$ where 
$\operatorname{ord}_{\Lambda_{i}} f^{s}=-m_{i} s-\mu_{i} / 2(i=0,1)$. Then we have, up to a constant muliiple,

$$
\begin{gathered}
b_{\Lambda_{0}}(s) / b_{\Lambda_{1}}(s)=\prod_{k=0}^{\nu}\left[\frac{1}{\nu+1}\left(\operatorname{ord}_{\Lambda_{1}} f^{s}-\operatorname{ord}_{\Lambda_{0}} f^{s}\right)+\frac{\mu+2 k}{2(\nu+\mu)}\right]^{\left(m_{0}-m_{1}\right) /(\nu+1)} \\
\text { where }[\alpha]^{k}=\alpha(\alpha+1) \cdots(\alpha+k-1) .
\end{gathered}
$$

Here we denote by $\operatorname{ord}_{\Lambda} f^{s}$ the order of $f^{s}$ at $\Lambda$ (See [2]). Note that $m_{0}$ and $m_{1}$ are non-negative integers, and $(\mu: \nu)=(1: 0)$ or $(\mu: \nu)$ is a pair of positive integers satisfying $\mu \geq 2, \nu \geq 1$, and $\left(m_{0}-m_{1}\right)$ is a multiple of $(\nu+1)$.

Corollary 1-2 ([2] Corollary 7-6). If $\Lambda_{0}$ and $\Lambda_{1}$ intersect regularly, i.e., $\mu=1$ and $\nu=0$, we have

$$
\begin{gathered}
b_{A_{0}}(s) / b_{A_{1}}(s)=\prod_{k=1}^{m_{0}-m_{1}}\left(\left(m_{0}-m_{1}\right) s+\frac{\mu_{0}-\mu_{1}-1}{2}+k\right) \\
\text { where } \operatorname{ord}_{A_{i}} f^{s}=-m_{i} s-\frac{\mu_{i}}{2} \quad(i=0,1) .
\end{gathered}
$$

Let $\Lambda$ be a good holonomic variety. Then $\Lambda=\overline{G\left(x_{0}, y_{0}\right)}$ for some $x_{0} \in V, y_{0} \in V^{*}$ where $G\left(x_{0}, y_{0}\right)=\left\{\left(\rho(g) x_{0}, \rho^{*}(g) y_{0}\right) ; g \in G\right\}$. In this case, we can calculate the order $\operatorname{ord}_{A} f^{s}$ by the following proposition.

Proposition 1-3 ([2] Proposition 4-14). Let $A_{0}$ be an element of the Lie algebra $g$ of $G$ satisfying $d \rho\left(A_{0}\right) x_{0}=0$ and $d \rho^{*}\left(A_{0}\right) y_{0}=y_{0}$. Then we have

$$
\operatorname{ord}_{A} f^{s}=s \delta \chi\left(A_{0}\right)-\operatorname{tr}_{V_{x_{0}}^{*}} d \rho_{x_{0}}\left(A_{0}\right)+\frac{1}{2} \operatorname{dim} V_{x_{0}}^{*}
$$

where $V_{x_{0}}^{*}$ denotes the conormal vector space $\left(d \rho(\mathfrak{g}) \cdot x_{0}\right)^{\perp}$, and $d \rho_{x_{0}}$ denotes the representation of $\mathrm{g}_{x_{0}}=\left\{A \in \mathfrak{g} ; d \rho(A) x_{0}=0\right\}$ induced by $d \rho^{*}$.

Now let $\Lambda_{0}=\overline{G\left(x_{0}, y_{0}\right)}$ and $\Lambda_{1}=\overline{G\left(x_{1}, y_{1}\right)}$ be good holonomic varieties such that $\left(x_{0}, y_{1}\right) \in \Lambda_{0} \cap \Lambda_{1}$ and $\operatorname{dim} G\left(x_{0}, y_{1}\right)=\operatorname{dim} V-1$. In this case, the intersection exponent $(\mu: \nu)$ is given by the following proposition.

Proposition 1-4 ([2] Proposition 6-5). Let $A_{1}$ be an element of $\mathfrak{g}$ satisfying $d \rho\left(A_{1}\right) x_{0}=0$ and $d \rho^{*}\left(A_{1}\right) y_{1}=y_{1}$. Then $A_{1}$ acts on the onedimensional vector space $\tilde{V}=V_{x_{0}}^{*}$ modulo $d \rho_{x_{0}}\left(\mathfrak{g}_{x_{0}}\right) y_{1}$. Let $\beta$ be its eigenvalue, i.e., $\beta=\operatorname{tr}_{\tilde{\nu}} A_{1}$. Then $\mu$ and $\nu$ are given by $\beta=\mu /(\mu+\nu),(\mu, \nu)=1$. If $\beta$ is not determined uniquely, i.e., $\beta$ depends on $A_{1}$, then we have $\mu=1$, $\nu=0$, and $A_{0}, A_{1}$ intersect regularly. 


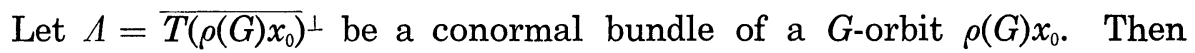
$G$ acts on $\Lambda$ prehomogeneously if and only if the colocalization $\left(G_{x_{0}}, \rho_{x_{0}}\right.$, $\left.V_{x_{0}}^{*}\right)$ at $x_{0}$ is a P.V. We shall consider some sufficient conditions that $\Lambda \subset W$, i.e., $\Lambda$ is a good holonomic variety.

Proposition 1-5 ([2] Proposition 6-6). Let $\Lambda_{0}$ and $\Lambda_{1}$ be two conormal bundles of some G-orbits. Assume that $\operatorname{dim} g_{0} \cdot p=\operatorname{dim} V-1$ for some $p \in \Lambda_{0} \cap \Lambda_{1}$ where $\mathfrak{g}_{0}=\{A \in \mathfrak{g} ; \delta \chi(A)=0\}$. Assume that $\Lambda_{0}\left(\right.$ or $\left.\Lambda_{1}\right) \subset W$. Then we have $\Lambda_{0} \cup \Lambda_{1} \subset W$. Moreover $W$ is non-singular and $W=\left\{(x, y) \in V \times V^{*}\right.$; $\langle d \rho(A) x, y\rangle=0$ for all $\left.A \in \mathfrak{g}_{0}\right\}$ near $p$.

Let $V_{x_{0}}=V \bmod d \rho(\mathfrak{g}) x_{0}$ be the normal vector space. Then the isotropy subgroup $G_{x_{0}}$ acts on $V_{x_{0}}$. We denote this action by $\tilde{\rho}_{x_{0}}$. Let $f_{x_{0}}$ be the localization of $f(x)$ at $x_{0}$ (See [2]). This is a relative invariant of $\left(G_{x_{0}}, \tilde{\rho}_{x_{0}}\right.$, $\left.V_{x_{0}}\right)$ corresponding to $\chi \mid G_{x_{0}}$. Let $S_{x_{0}}$ be the singular set of $\left(G_{x_{0}}, \tilde{\rho}_{x_{0}}, V_{x_{0}}\right)$.

Proposition 1-6 ([2] Proposition 6-9). If $\operatorname{grad} \log f_{x_{0}}: V_{x_{0}}-S_{x_{0}} \rightarrow V_{x_{0}}^{*}$ is generically surjective, then $\Lambda_{0}=\overline{T\left(\rho(G) x_{0}\right)^{\perp}} \subset W$, i.e., $\Lambda_{0}$ is a good holonomic variety.

Corollary 1-7 ([2] Corollary 6-10). Assume that the colocalization $\left(G_{x_{0}}, \rho_{x_{0}}, V_{x_{0}}^{*}\right)$ of $(G, \rho, V)$ at $x_{0}(\in V)$ is a regular P.V. If $\delta \chi \mid g_{x_{0}}$ is a nondegenerate element, then the conormal bundle $\Lambda_{0}=\overline{T\left(\rho(G) x_{0}\right)^{\perp}}$ of the G-orbit $\rho(G) x_{0}$ is a good holonomic variety.

Corollary 1-8 ([2] Corollary 6-11). Assume that the colocalization $\left(G_{x_{0}}, \rho_{x_{0}}, V_{x_{0}}^{*}\right)$ of $(G, \rho, V)$ at $x_{0}(\in V)$ is an irreducible regular $P . V$. Then the conormal bundle $\Lambda_{0}=\overline{T\left(\rho(G) x_{0}\right)^{\perp}}$ of the orbit $\rho(G) x_{0}$ is a good holonomic variety.

Proposition 1-9 ([1] Proposition 14 in $\S 4$ ).

(1) For $d=\operatorname{deg} f$ and $n=\operatorname{dim} V$, we have $d \mid 2 n$ and $\chi(g)^{2 n / d}=$ $\operatorname{det}_{V} \rho(g)^{2}$ for $g \in G$.

(2) $\delta \chi(A)=(d / n) \operatorname{tr} d \rho(A)$ for $A \in \mathfrak{g}$.

Remark 1-10. Let $(G, \rho, V)$ be an irreducible regular P.V. with finitely many orbits. Let $\mathscr{L}=\left\{\Lambda, \Lambda^{\prime}, \cdots, \Lambda^{\prime \prime}\right\}$ be the set of all conormal bundles in $W$, of some $G$-orbits in $V$. The holonomy diagram is, by definition, given as follows.

If $\operatorname{dim} \Lambda \cap \Lambda^{\prime}=\operatorname{dim} V-1$, and $\Lambda \cap \Lambda^{\prime} \not \subset \Lambda^{\prime \prime}$ for any other $\Lambda^{\prime \prime}$ in $\mathscr{L}$, then we write the diagram as in Figure 1-1. Moreover, if $\Lambda$ and $\Lambda^{\prime}$ are good 


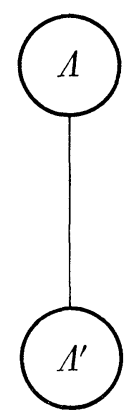

Figure 1-1.

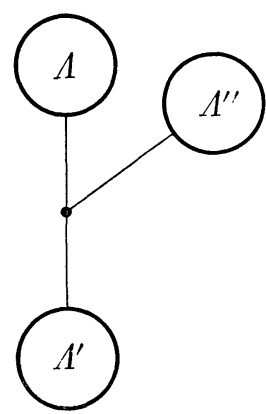

Figure 1-2.

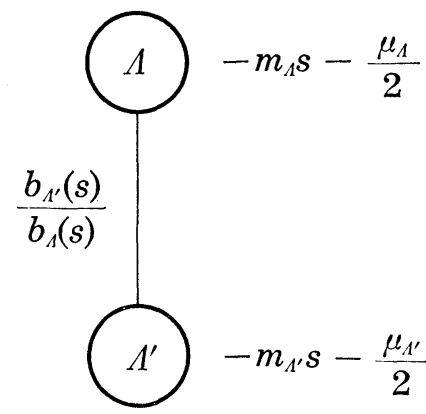

Figure 1-3. $\left(m_{A^{\prime}}>m_{A}\right)$

holonomic varieties, we write the orders $\operatorname{ord}_{\Lambda} f^{s}=-m_{\Lambda} s-\mu_{\Lambda} / 2$ for $\Lambda$ and $\Lambda^{\prime}$, and the ratio of the $b$-functions as in Figure $1-3$. If $\operatorname{dim} \Lambda \cap \Lambda^{\prime}=\operatorname{dim}$ $V-1$ and $\Lambda \cap \Lambda^{\prime} \subset \Lambda^{\prime \prime}$ for some $\Lambda^{\prime \prime}$, then we write the diagram as in Figure 1-2 (e.g. Figure 11-1). Although some general theory for such cases has been established, it is not published yet and hence in this paper we avoid to argue this case. Actually, only in $\S 11$, such case will appear and to calculate the $b$-function in $\S 11$, we can use another part of the holonomy diagram. Although usually we do not write the conormal bundles outside $W$ (e.g. Figure 3-2), sometimes we write them (e.g. Figure 4-1, Figure 11-1). Since $G$ is reductive, we have $(G, \rho, V) \cong\left(G, \rho^{*}, V^{*}\right)$ and we identify them.

We sometimes write as (A) when $T$ and $T^{\prime}$ are the dual orbits of each other (See $\S 11$ ) where $\Lambda$ and $\Lambda^{\prime}$ are the conormal bundles of $T$ and $T^{\prime}$ respectively. If $T=T^{\prime}$, we write as (1).g. Figure $4-1$ and Figure 11-1).

\section{§ 2. Regular P.V.'s related with $G L(n)$}

We shall use the same notations as in [1].

2-1. $\left(\tilde{G} \times G L(m), \tilde{\rho} \otimes \Lambda_{1}, V(m) \otimes V(m)\right)$ where $\tilde{\rho}: \tilde{G} \rightarrow G L(V(m))$ is an $m$-dimensional irreducible representation of a connected semi-simple algebraic group $\tilde{G}$ (or $\tilde{G}=\{1\}$ and $m=1$ )

The representation space $V=V(m) \otimes V(m)$ can be identified with the totality of $m \times m$ matrices $M(m, C)$. Then the action $\rho=\tilde{\rho} \otimes \Lambda_{1}$ is given by $\rho(g) X=\tilde{\rho}\left(g_{1}\right) X^{t} g_{2}$ for $g=\left(g_{1}, g_{2}\right) \in G=\tilde{G} \times G L(m), X \in M(m, C)$. The relative invariant $f(X)$ is given by the determinant: $f(X)=\operatorname{det} X$. Since we are concerned with relative invariants, we may assume that $\tilde{G}=S L(m)$ and $\tilde{\rho}=\Lambda_{1}$. It is well-known that there exist $(m+1)$-orbits 


$$
\begin{aligned}
& \rho(G) X_{\mu}=\{X \in M(m, C) ; \operatorname{rank} X=\mu\}
\end{aligned}
$$

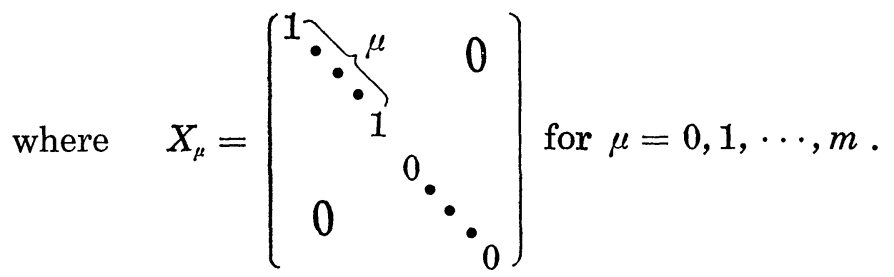

We identify the dual $V^{*}$ of $V$ with $V=M(m, C)$ by $\langle X, Y\rangle=\operatorname{tr}^{t} X Y$ for $X, Y \in M(m, C)$. Then the dual $\rho^{*}$ of $\rho$ is given by $\rho^{*}(g) Y={ }^{t} g_{1}^{-1} Y g_{2}^{-1}$ for $g=\left(g_{1}, g_{2}\right) \in G=S L(m) \times G L(m), Y \in M(m, C)$.

Since $d \rho(\tilde{A}) X_{\mu}=A X_{\mu}+X_{\mu}{ }^{t} B=\left(\frac{A_{1}+{ }^{t} B_{1}}{A_{3}} \mid \frac{{ }^{t} B_{3}}{0}\right)$ for $\tilde{A}=(A, B) \in g$ with $A=\left(\begin{array}{ll}A_{1} & A_{2} \\ A_{3} & A_{4}\end{array}\right)$ and $B=\left(\begin{array}{ll}B_{1} & B_{2} \\ B_{3} & B_{4}\end{array}\right)$, the conormal vector space $V_{X^{\mu}}^{*}=$ $\left(d \rho(\mathfrak{g}) X_{\mu}\right)^{\perp}$ is given by $V_{X^{\mu}}^{*}=\left\{\left(\begin{array}{ll}0 & 0 \\ 0 & Y_{\mu}\end{array}\right) ; Y_{\mu} \in M(m-\mu, C)\right\}$. The isotropy subalgebra $\mathfrak{g}_{X \mu}=\left\{\tilde{A} \in \mathfrak{g} ; d \rho(\tilde{A}) X_{\mu}=0\right\}$ is given by

$$
\begin{aligned}
\mathfrak{g}_{X \mu}= & \left\{\left(\left(\begin{array}{cc}
A_{1} & A_{2} \\
0 & A_{4}
\end{array}\right),\left(\begin{array}{cc}
-{ }^{t} A_{1} & B_{2} \\
0 & B_{4}
\end{array}\right)\right) \in \mathfrak{g} ; A_{1} \in M(\mu, C), A_{2}, B_{2}\right. \\
& \left.\in M(\mu, m-\mu, C), A_{4}, B_{4} \in M(m-\mu, C)\right\} .
\end{aligned}
$$

This $\mathfrak{g}_{X \mu}$ acts on $V_{X_{\mu}}^{*}$ as $d \rho_{X_{\mu}}(\tilde{A}) Y_{\mu}=-{ }^{t} A_{4} Y_{\mu}-Y_{\mu} B_{4}$ for $\tilde{A} \in \mathfrak{g}_{X \mu}$. Therefore we have $\left(G_{X_{\mu}}, \rho_{X \mu}, V_{X_{\mu}}^{*}\right) \cong\left(S L(m-\mu) \times G L(m-\mu), \Lambda_{1} \otimes \Lambda_{1}\right.$, $V(m-\mu) \otimes V(m-\mu))$. Put $Y_{\mu}=\left(\begin{array}{cc}0 & 0 \\ 0 & I_{m-\mu}\end{array}\right)$ where $I_{m-\mu}$ denotes the unit matrix of size $m-\mu(\mu=0,1, \cdots, m)$. Then $Y_{\mu}$ is a generic point of the colocalization $\left(G_{X_{\mu}}, \rho_{X_{\mu}}, V_{X_{\mu}}^{*}\right)$, and $Y_{\mu+1}$ is a point of the one-codimensional orbit $(\mu \leq m-1)$. We denote by $\Lambda_{\mu}$ the conormal bundle of $\rho(G) X_{\mu}(0 \leq$ $\mu \leq m)$. Then, we have $\operatorname{dim} \Lambda_{\mu} \cap \Lambda_{\mu+1}=\operatorname{dim} V-1$. Note that the colocalization $\left(G_{X_{\mu}}, \rho_{X_{\mu}}, V_{X_{\mu}}^{*}\right)(\mu=0,1, \cdots, m)$ has finitely many orbits with the unique one-codimensional orbit, it is clear that we have obtained all onecodimensional intersections among $\Lambda_{\mu}(\mu=0,1, \cdots, m)$. Since $\mathfrak{g}_{0}=\mathfrak{g l}(m) \oplus$ $\mathcal{I l}(m)$, we have $\operatorname{dim} \mathfrak{g}_{0}\left(X_{\mu}, Y_{\mu+1}\right)=m^{2}-1$ for $\mu=0,1, \cdots, m-1$, and hence by Proposition 1-5, we have $\Lambda_{\mu} \subset W$, i.e., $\Lambda_{\mu}$ is a good holonomic variety $(0 \leqq \mu \leqq m)$. Note that $\Lambda_{m}=V \times\{0\}$ is always a good holonomic variety. We shall calculate the intersection exponent $(\tilde{\mu}: \tilde{\nu})$ of $\Lambda_{\mu}$ and $\Lambda_{\mu+1}$ by using

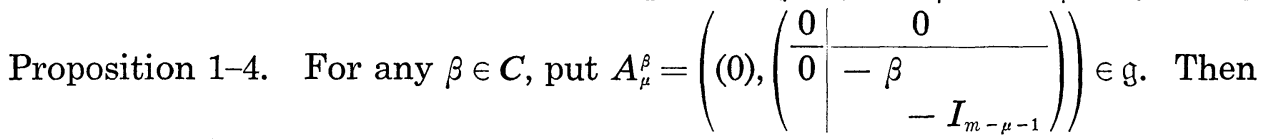
we have $d \rho\left(A_{\mu}^{\beta}\right) X_{\mu}=0, d \rho^{*}\left(A_{\mu}^{\beta}\right) Y_{\mu+1}=Y_{\mu+1}$, and $\beta=\operatorname{tr} A_{\mu}^{\beta}$ where $\operatorname{tr}$ denotes 
the trace of $A_{\mu}^{\beta}$ on $V_{X_{\mu}}^{*}$ modulo $d \rho_{X_{\mu}}\left(\mathfrak{g}_{X_{\mu}}\right) Y_{\mu+1}$ since $V_{X_{\mu}}^{*}$ modulo $d \rho_{X_{\mu}}\left(\mathfrak{g}_{X_{\mu}}\right) Y_{\mu+1}$ $\cong\left\{y E_{\mu+1, \mu+1} \in M(m, C) ; y \in C\right\}$ where $E_{i j}$ denotes the matrix unit. Therefore we have $\tilde{\mu}=1$ and $\tilde{\nu}=0$, i.e., they intersect regularly. Now by Proposition $1-3$, we shall calculate the order $\operatorname{ord}_{\Lambda_{\mu}} f^{s}$ of $\mathfrak{m}=\mathscr{E} f^{s}$ at $\Lambda_{\mu}$ where $f(X)=$ $\operatorname{det} X$.

Put $A_{\mu}=\left((0),\left(\begin{array}{cc}0 & 0 \\ 0 & -I_{m-\mu}\end{array}\right)\right) \in \mathfrak{g}$. Then $d \rho\left(A_{\mu}\right) X_{\mu}=0$ and $d \rho^{*}\left(A_{\mu}\right) Y_{\mu}=$ $Y_{\mu}(0 \leq \mu \leq m)$. The character $\delta \chi$ corresponding to $f(X)=\operatorname{det} X$ is given by $\delta \chi(\tilde{A})=\operatorname{tr} B$ for $\tilde{A}=(A, B) \in \mathfrak{g}=\mathfrak{g l}(m) \oplus \operatorname{gr}(m)$. Since $\operatorname{dim} V_{X_{\mu}}^{*}=(m-\mu)^{2}$ and $\operatorname{tr}_{V_{X_{\mu}}^{*}} d \rho_{X_{\mu}}\left(A_{\mu}\right)=(m-\mu)^{2}$, we have $\operatorname{ord}_{A_{\mu}} f^{s}=s \delta \chi\left(A_{\mu}\right)-\operatorname{tr}_{V_{X \mu}^{*}} d \rho_{X_{\mu}}\left(A_{\mu}\right)+$ $(1 / 2) \operatorname{dim} V_{X_{\mu}}^{*}=-(m-\mu) s-\left((m-\mu)^{2} / 2\right)$. Thus we obtain the holonomy diagram (Figure 2-1).

By Corollary 1-2, we have $b_{A_{\mu}}(s) / b_{A_{\mu+1}}(s)=s+(m-\mu)(0 \leq \mu \leq m-1)$. Hence

$$
\begin{aligned}
b(s) & =b_{\Lambda_{0}}(s)=b_{\Lambda_{m}}(s) \cdot \prod_{\mu=0}^{m-1} b_{\Lambda_{\mu}}(s) / b_{\Lambda_{\mu+1}}(s) \\
& =\prod_{\mu=0}^{m-1}(s+m-\mu)=(s+1)(s+2) \cdots(s+m) .
\end{aligned}
$$

Note that $b_{A_{m}}(s)=1$.

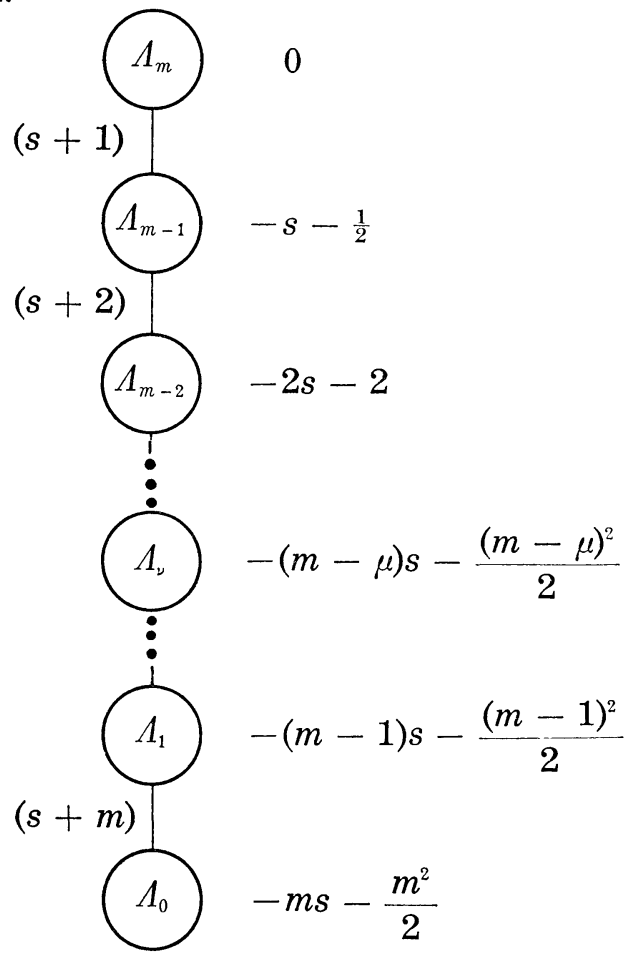

Figure 2-1. Holonomy diagram of $\left(S L(m) \times G L(m), \Lambda_{1} \otimes \Lambda_{1}, V(m) \otimes V(m)\right)$ 
Remark 2-1. The $b$-function of $\mathbf{2 - 1}$ is classically known by using Capelli's identity (See H. Weyl: Classical Groups).

2-2. $\left(G L(n), 2 \Lambda_{1}, V\left(\frac{1}{2} n(n+1)\right)\right)(n \geq 2)$

The representation space can be identified with the totality of $n \times n$ symmetric matrices $V=\left\{X \in M(n, C) ;{ }^{t} X=X\right\}$. Then the action $\rho=2 \Lambda_{1}$ of $G L(n)$ on $V$ is given by $\rho(g) X=g X^{t} g$ for $g \in G L(n), X \in V$. It is wellknown that there exist $(n+1)$-orbits $\rho(G) X_{\nu}=\{X \in V$; rank $X=\nu\}$ where

$$
X_{\nu}=\left(\begin{array}{cccc}
1 . & & & \\
& \ddots & & 0 \\
& 1 & & \\
& & 0 & \\
0 & & & \ddots \\
& & &
\end{array}\right) \text { for } \nu=0,1, \cdots, n
$$

The relative invariant is given by the determinant $f(X)=\operatorname{det} X$. If we identify the dual $V^{*}$ of $V$ with $V$ by $\langle X, Y\rangle=\operatorname{tr} X Y$, we have $\rho^{*}(g) Y=$ ${ }^{t} g^{-1} Y g^{-1}$ for $g \in G L(n)$. We have

$$
\begin{gathered}
d \rho(A) X_{\nu}=A X_{\nu}+X_{\nu}{ }^{t} A=\left(\frac{A_{1}+{ }^{t} A_{1}}{A_{3}} \mid \frac{{ }^{t} A_{3}}{0}\right) \\
\text { for } A=\left(\begin{array}{ll}
A_{1} & A_{2} \\
A_{3} & A_{4}
\end{array}\right) \in \mathfrak{g l}(n) .
\end{gathered}
$$

Therefore we have

$$
\begin{aligned}
\mathrm{g}_{X_{\nu}}= & \left\{\left(\begin{array}{cc}
A_{1} & A_{2} \\
0 & A_{4}
\end{array}\right) ;{ }^{t} A_{1}=-A_{1}, A_{1} \in M(\nu), A_{2} \in M(\nu, n-\nu),\right. \\
& \left.A_{4} \in M(n-\nu)\right\} .
\end{aligned}
$$

Since $\operatorname{dim} \mathfrak{g}_{X_{\nu}}=n(n-\nu)+(\nu(\nu-1) / 2)$, we have $\operatorname{dim} \rho(G) X_{\nu}=n \nu-$ $(\nu(\nu-1) / 2)$. The conormal vector space $V_{X_{\nu}}^{*}$ is given by

$$
V_{X_{\nu}}^{*}=\left\{\left(\begin{array}{cc}
0 & 0 \\
0 & W_{\nu}
\end{array}\right) ;{ }^{t} W_{\nu}=W_{\nu} \in M(n-\nu)\right\} .
$$

Since $d \rho^{*}\left(\left(\begin{array}{cc}A_{1} & A_{2} \\ 0 & A_{4}\end{array}\right)\right)\left(\begin{array}{cc}0 & 0 \\ 0 & W_{\nu}\end{array}\right)=\left(\frac{0}{0} \mid \frac{0}{-{ }^{t} A_{4} W-W A_{4}}\right)$, the colocalization $\left(G_{X_{\nu}}, \rho_{X_{\nu}}, V_{X_{\nu}}^{*}\right)$ at $X_{\nu}$ is isomorphic to $\left(G L(n-\nu), 2 \Lambda_{1}, V\left(\frac{1}{2}(n-\nu)(n-\nu+1)\right)\right)$. Put $Y_{\nu}=\left(\begin{array}{cc}0 & 0 \\ 0 & I_{n-\nu}\end{array}\right)(\nu=0,1, \cdots, n)$. Then $Y_{\nu}$ is a generic point of the 
colocalization at $X_{\nu}$ and $Y_{\nu+1}$ is a point of the unique one-codimensional orbit. Thus we have $\operatorname{dim} \Lambda_{\nu} \cap \Lambda_{\nu+1}=\operatorname{dim} V-1$, where $\Lambda_{\nu}$ denotes the conormal bundle of $\rho(G) X_{\nu}$. Since $\operatorname{dim} d \rho^{*}\left(\mathfrak{g}_{X_{\nu}} \cap \mathfrak{g}_{0}\right) Y_{\nu+1}=\operatorname{dim} d \rho^{*}\left(\mathfrak{g}_{X_{\nu}}\right) Y_{\nu+1}$, we have $\operatorname{dim} g_{0}\left(X_{\nu}, Y_{\nu+1}\right)=(n(n+1) / 2)-1$, and hence $\Lambda_{\nu}$ is a good holonomic variety by Proposition $1-5(\nu=0,1, \cdots, n)$.

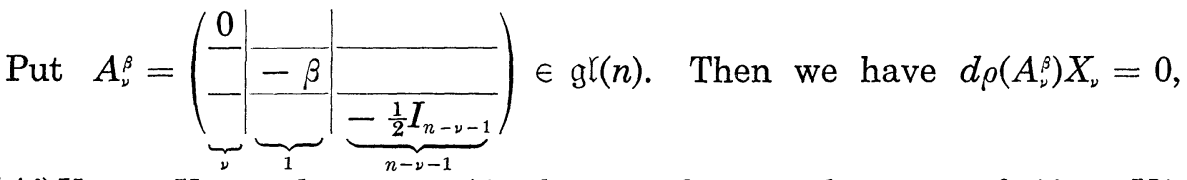
$d \rho^{*}\left(A_{\nu}^{\beta}\right) Y_{\nu+1}=Y_{\nu+1}$ and $2 \beta=\operatorname{tr} A_{\nu}^{\beta}$ where tr denotes the trace of $A_{\nu}^{\beta}$ on $V_{X_{\nu}}^{*}$ modulo $d \rho_{X_{\nu}}\left(g_{X_{\nu}}\right) Y_{\nu+1}$. Hence, $\Lambda_{\nu}$ and $\Lambda_{\nu+1}$ intersect regularly, i.e., the intersection exponent of $\Lambda_{\nu}$ and $\Lambda_{\nu+1}$ equals $(1: 0)$. We shall calculate the order $\operatorname{ord}_{A_{\nu}} f^{s}$ by Proposition 1-3. Put $A_{\nu}=\left(\begin{array}{cc}0 & 0 \\ 0 & -\frac{1}{2} I_{n-\nu}\end{array}\right)(0 \leq \nu \leq n)$. Then $d \rho\left(A_{\nu}\right) X_{\nu}=0$ and $d \rho^{*}\left(A_{\nu}\right) Y_{\nu}=Y_{\nu}$. Since $\delta \chi\left(A_{\nu}\right)=2 \operatorname{tr} A_{\nu}=-(n-\nu)$, and $\operatorname{tr}_{V_{X_{\nu}}^{*}} d \rho_{X_{\nu}}\left(A_{\nu}\right)=\operatorname{dim} V_{X_{\nu}}^{*}=\frac{1}{2}(n-\nu)(n-\nu+1)$, we have ord $A_{A_{\nu}} f^{s}=s \delta \gamma\left(A_{\nu}\right)$ $-\operatorname{tr}_{V_{X_{\nu}}^{*}} d \rho_{X_{\nu}}\left(A_{\nu}\right)+\frac{1}{2} \operatorname{dim} V_{X_{\nu}}^{*}=-(n-\nu) s-\frac{1}{4}(n-\nu)(n-\nu+1)$.

Thus we obtain the holonomy diagram (Figure 2-2). By Corollary

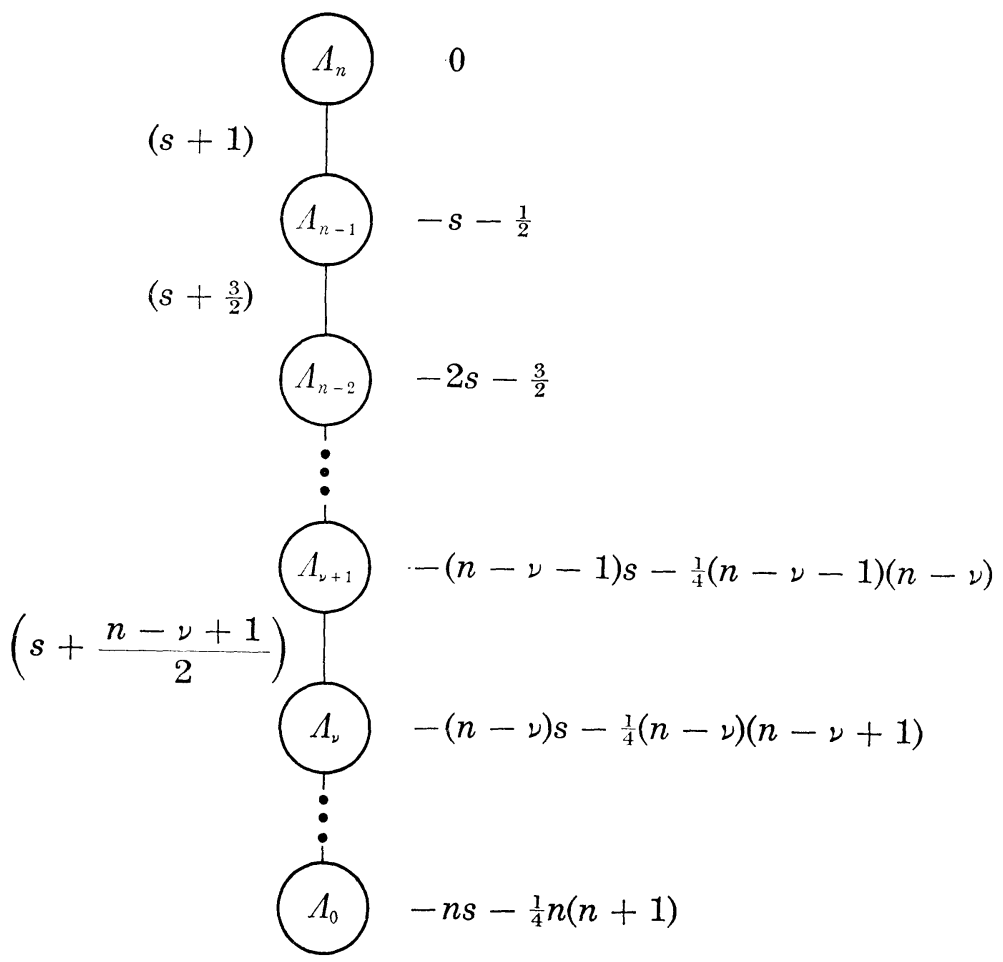

Figure 2-2. Holonomy diagram of $\left(G L(n), 2 \Lambda_{1}, V\left(\frac{1}{2} n(n+1)\right)\right) \quad(n \geq 2)$ 
1-2, we have $b_{\Lambda_{\nu}}(s) / b_{\Lambda_{\nu+1}}(s)=s+((n-\nu+1) / 2)(0 \leq \nu \leq n-1)$, and hence $b(s)=b_{\Lambda_{0}}(s)=\prod_{\nu=1}^{n}(s+(\nu+1) / 2)$.

Remark. The $b$-function of 2-2 is also already known. It can be obtained by using Capelli's identity or by a direct calculation of the Fourier transform of $f(x)^{s}$.

2-3. $\quad\left(G L(2 m), \Lambda_{2}, V(m(2 m-1))\right) \quad(m \geq 3)$

The representation space can be identified with $V_{m}=\{X \in M(2 m, C) \mid$ $\left.{ }^{t} X=-X\right\}$. Then the action $\rho=\Lambda_{2}$ is given by $\rho(g) X=g X^{t} g$ for $g \in$ $G L(2 m), X \in V_{m}$. The relative invariant $f(X)$ is the Pfaffian of $X$. It is well-known that there exists $(m+1)$-orbits $\rho(G) X_{\mu}=\left\{X \in V_{m} ; \operatorname{rank} X=2 \mu\right\}$ where $X_{\mu}=\left[\begin{array}{cccc}0 & 0 & I_{\mu} & 0 \\ 0 & 0 & 0 & 0 \\ -I_{\mu} & 0 & 0 & 0 \\ 0 & 0 & 0 & 0\end{array}\right] \quad(0 \leq \mu \leq m)$.

By simple calculation, we have

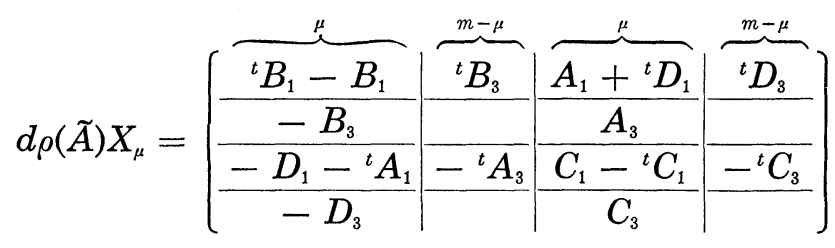

$$
\begin{aligned}
& \text { where } \tilde{A}=\left(\begin{array}{cc|cc}
A_{1} & A_{2} & B_{1} & B_{2} \\
A_{3} & A_{4} & B_{3} & B_{4} \\
\hline C_{1} & C_{2} & D_{1} & D_{2} \\
C_{3} & C_{4} & D_{3} & D_{4}
\end{array}\right)
\end{aligned}
$$

and hence,

$$
\begin{aligned}
& \mathfrak{g}_{X_{\mu}}=\left\{\left(\begin{array}{cc|cc}
A_{1} & A_{2} & B_{1} & B_{2} \\
0 & A_{4} & 0 & B_{4} \\
\hline C_{1} & C_{2} & -{ }^{t} A_{1} & D_{2} \\
0 & C_{4} & 0 & D_{4}
\end{array}\right] ;{ }^{t} B_{1}=B_{1},{ }^{t} C_{1}=C_{1}\right\}
\end{aligned}
$$

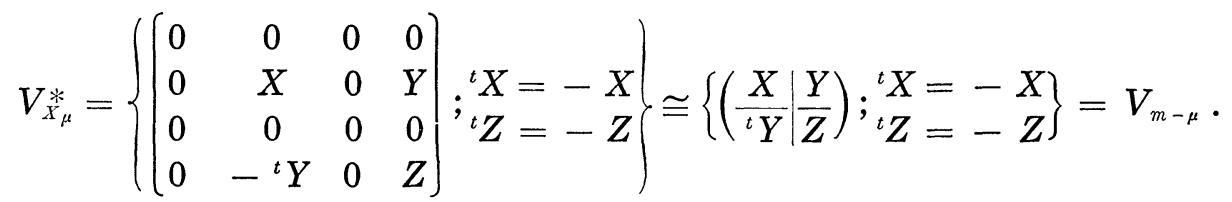

Since $\mathfrak{g}_{X_{\mu}}$ acts on $V_{\tilde{X}_{, \mu}}^{*}$ as $\tilde{X} \mapsto-{ }^{t} \tilde{A_{4}} \tilde{X}-\tilde{X} \tilde{A}_{4}$ where $\tilde{A}_{4}=\left(\frac{A_{4}}{C_{4}} \mid \frac{B_{4}}{D_{4}}\right)$ and 
$\tilde{X}=\left(\frac{X}{-{ }^{t} Y} \mid \frac{Y}{Z}\right)$ with ${ }^{t} X=-X,{ }^{t} Z=-Z$, the colocalization $\left(G_{X_{\mu}}, \rho_{X_{\mu}}, V_{X_{\mu}}^{*}\right)$ at $X_{\mu}$ is isomorphic to $\left(G L(2 m-2 \mu), \Lambda_{2}, V((m-\mu)(2 m-2 \mu-1))\right)$. Here we identified the dual $V_{m}^{*}$ of $V_{m}$ with $V_{m}$ by $\langle X, Y\rangle=\operatorname{tr} X Y$.

$$
\text { Put } Y_{\mu}=\left(\begin{array}{cccc}
0 & 0 & 0 & 0 \\
0 & 0 & 0 & I_{m-\mu} \\
0 & 0 & 0 & 0 \\
0 & -I_{m-\mu} & 0 & 0
\end{array}\right) \quad(0 \leq \mu \leq m) \text {. }
$$

Then $Y_{\mu}$ is a generic point of the colocalization $\left(G_{X_{\mu}}, \rho_{X_{\mu}}, V_{X_{\mu}}^{*}\right)$ at $X_{\mu}$, and $Y_{\mu+1}$ is a point of the one-dimensional orbit and hence we have $\operatorname{dim} \Lambda_{\mu} \cap$ $\Lambda_{\mu+1}=\operatorname{dim} V-1(0 \leq \mu \leq m-1)$ where $\Lambda_{\mu}$ denotes the conormal bundle of $\rho(G) X_{\mu}$.

By (2.6), we have $\mathfrak{g}_{X_{\mu}} \not \subset \mathfrak{g}_{0}$ for $\mu \neq m$, and hence $\operatorname{dim} d \rho(\mathrm{g}) X_{\mu}=$ $\operatorname{dim} d_{\rho}\left(\mathfrak{g}_{0}\right) X_{\mu}$ for $\mu \neq m$. Applying this fact to the colocalization at $X_{\mu}$, we have $\operatorname{dim} \mathrm{g}_{0}\left(X_{\mu}, Y_{\mu+1}\right)=\operatorname{dim} \mathrm{g}\left(X_{\mu}, Y_{\mu+1}\right)=m(2 m-1)-1$. This implies that $\Lambda_{\mu}$ is a good holonomic variety by Proposition 1-5.

$$
\text { Put } A_{\mu}^{\beta}=\left(\begin{array}{c|c|c|c}
\hline 0 & 0 & 0 & 0 \\
\hline 0 & -\beta-\frac{1}{2} I_{m-\mu-1} & 0 & 0 \\
\hdashline 0 & 0 & 0 & 0 \\
\hline 0 & 0 & 0 & -\beta-\frac{1}{2} I_{m-\mu-1}
\end{array}\right) \quad \text { for } \beta \in C \text {. }
$$

Then we have $d \rho\left(A_{\mu}^{\beta}\right) X_{\mu}=0, d \rho^{*}\left(A_{\mu}^{\beta}\right) Y_{\mu+1}=Y_{\mu+1}$ and $\operatorname{tr} A_{\mu}^{\beta}=2 \beta$ where tr denotes the trace of $A_{\mu}^{\beta}$ on $V_{X_{\mu}}^{*}$ modulo $d \rho\left(g_{X_{\mu}}\right) Y_{\mu+1}$, and hence by Proposition 1-4, $\Lambda_{\mu}$ and $\Lambda_{\mu+1}$ intersect regularly, i.e., the intersection exponent of $\Lambda_{\mu}$ and $\Lambda_{\mu+1}$ equals $(1: 0)$. We shall calculate the order $\operatorname{ord}_{\Lambda_{\mu}} f^{s}$.

Put $\quad A_{0}=\left(\begin{array}{l|l|l|l}- & & & \\ \hline-\frac{1}{2} I_{m-\mu} & - & \\ \hline\end{array}\right)$. Then we have $d \rho\left(A_{0}\right) X_{\mu}=0$ and $d \rho^{*}\left(A_{0}\right) Y_{\mu}=Y_{\mu} . \quad$ Since $\delta \chi\left(A_{0}\right)=-(m-\mu), \operatorname{tr}_{V_{X_{\mu}}^{*}} A_{0}=\operatorname{dim} V_{\tilde{X}_{\mu}}^{*}=(m-\mu)$ $(2 m-2 \mu-1)$, we have ord ${ }_{A_{\mu}} f^{s}=s \delta \chi\left(A_{0}\right)-\operatorname{tr}_{V_{X_{\mu}}^{*}} A_{0}+\frac{1}{2} \operatorname{dim} V_{X_{\mu}}^{*}=-(m-\mu) s$ $-\frac{1}{2}(m-\mu)(2 m-2 \mu-1)$.

By Corollary 1-2, we have $b_{\mu_{\mu}}(s) / b_{\mu_{\mu+1}}(s)=s+2(m-\mu)-1(0 \leq \mu \leq$ $m-1$ ). Hence we obtain the holonomy diagram (Figure 2-3) and $b$-function $b(s)=\prod_{\mu=0}^{m-1}(s+2(m-\mu)-1)=\prod_{k=1}^{m}(s+2 k-1)$. 


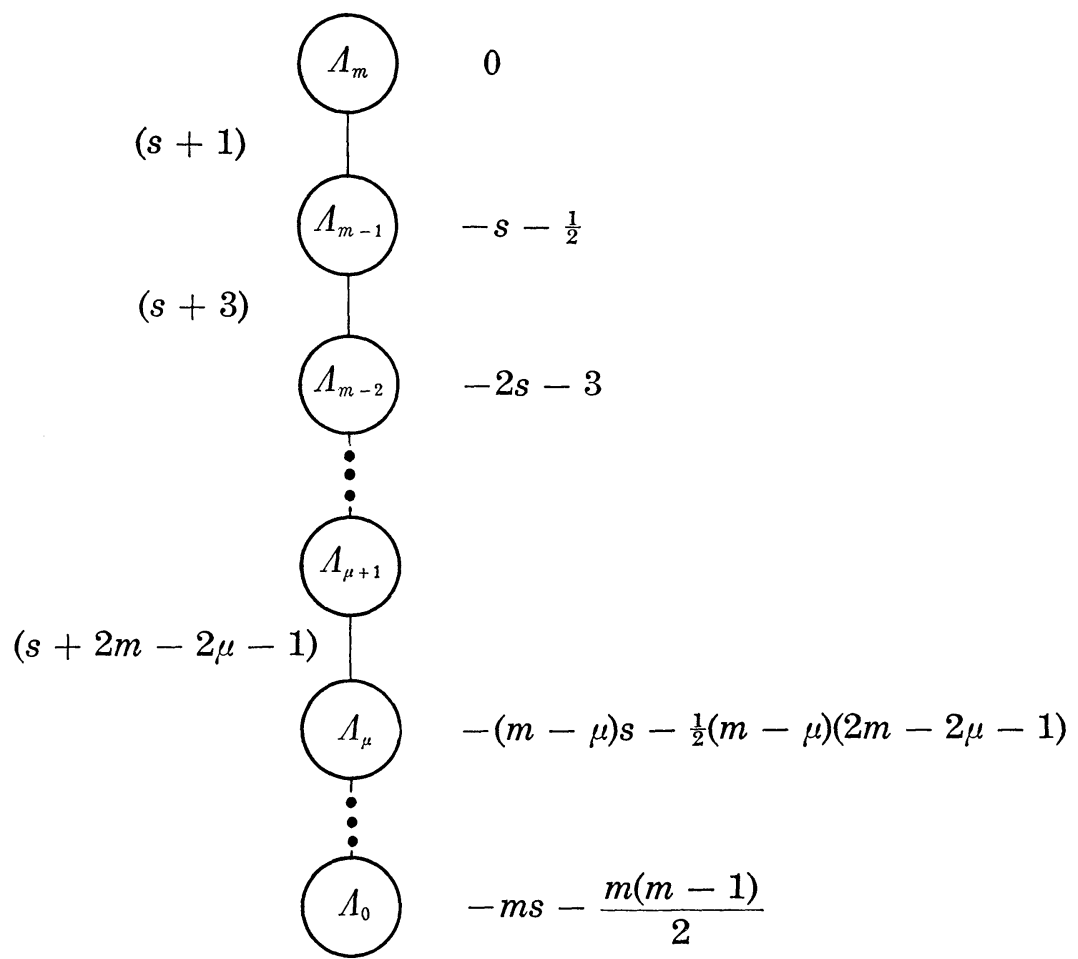

Figure 2-3. Holonomy diagram of $\left(G L(2 m), \Lambda_{2}, V(m(2 m-1))\right) \quad(m \geq 3)$.

Remark. These three P.V.'s have many common properties: $\left(G L(m), 2 \Lambda_{1}, V\left(\left(\begin{array}{c}m \\ 2\end{array}\right) \ell+m\right)\right)$ with $\ell \doteq 1 \quad$ (2) $\left(S L(m) \times G L(m), \Lambda_{1} \otimes \Lambda_{1}\right.$, $\left.V\left(\left(\begin{array}{c}m \\ 2\end{array}\right) \ell+m\right)\right)$ with $\ell=2$ (3) $\left(G L(2 m), \Lambda_{2}, V\left(\left(\begin{array}{c}m \\ 2\end{array}\right) \ell+m\right)\right)$ with $\ell=4$. They have $(m+1)$-orbits and their relative invariants are of degree $m$ of $\left(\begin{array}{c}m \\ 2\end{array}\right) \ell+m$ variables. We denote (A) by $(\mu$ if $\Lambda$ is the conormal bundle of a $\mu$-codimensional orbit. Then their holonomy diagrams are as in Figure $2-4$.

§3. $\left(S p(n) \times G L(2 m), \Lambda_{1} \otimes \Lambda_{1}, V(2 n) \otimes V(2 m)\right)$ with $n \geq 2 m$

The representation space $V$ can be identified with the totality of $2 n \times 2 m$ matrices. Then the action $\rho=\Lambda_{1} \otimes \Lambda_{1}$ is given by $\rho(g) X=g_{1} X^{t} g_{2}$ for $g=\left(g_{1}, g_{2}\right) \in G=S p(n) \times G L(2 m), X \in V$. Let $X$ be an element of $V$ such that $\operatorname{rank} X=\nu$ and $\operatorname{rank}^{t} X J X=2 \mu(2 m \geqq \nu \geqq 2 \mu \geqq 0)$ where $J=$ $\left(\begin{array}{cc}0 & -I_{n} \\ I_{n} & 0\end{array}\right)$. Then by the action of $G L(2 m)$, we may assume that $X=\left(X^{\prime}, 0\right)$ with $X^{\prime} \in M(2 n, \nu)$ satisfying ${ }^{t} X^{\prime} J X^{\prime}=\left(\begin{array}{ccc}0 & 0 & I_{\mu} \\ 0 & 0 & 0 \\ -I_{\mu} & 0 & 0\end{array}\right)$. Put $X_{\nu, 2 \mu}$ as follows. 


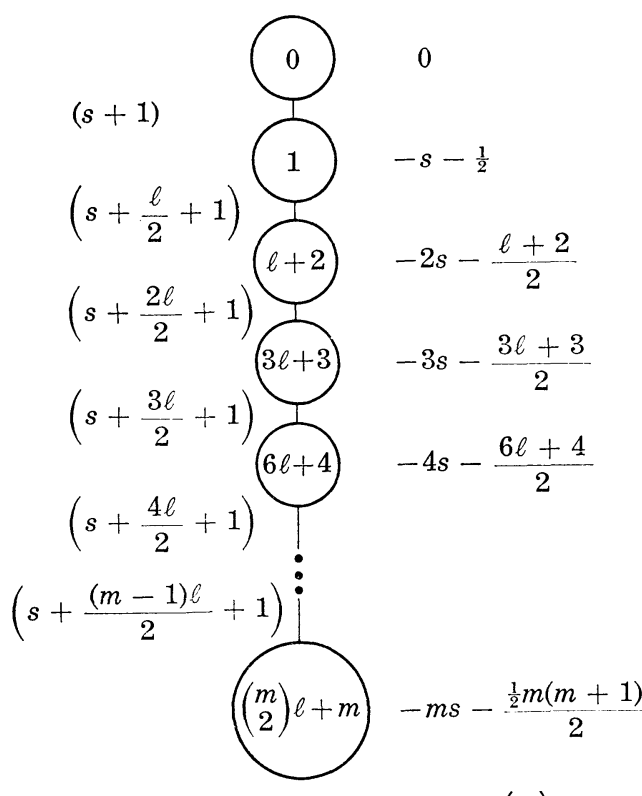

$(m+1)$-orbits $\operatorname{deg} f=m \quad \operatorname{dim} V=\left(\begin{array}{c}m \\ 2\end{array}\right) \ell+m$

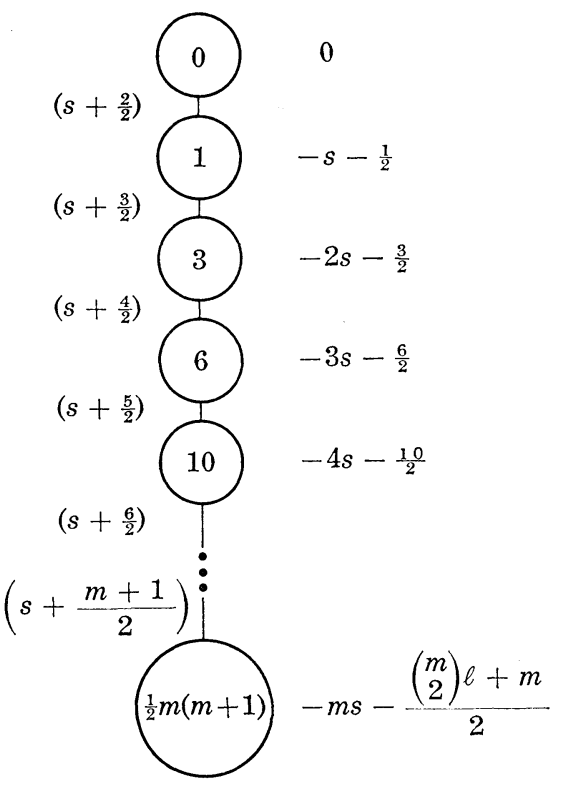

(1) $\ell=1$

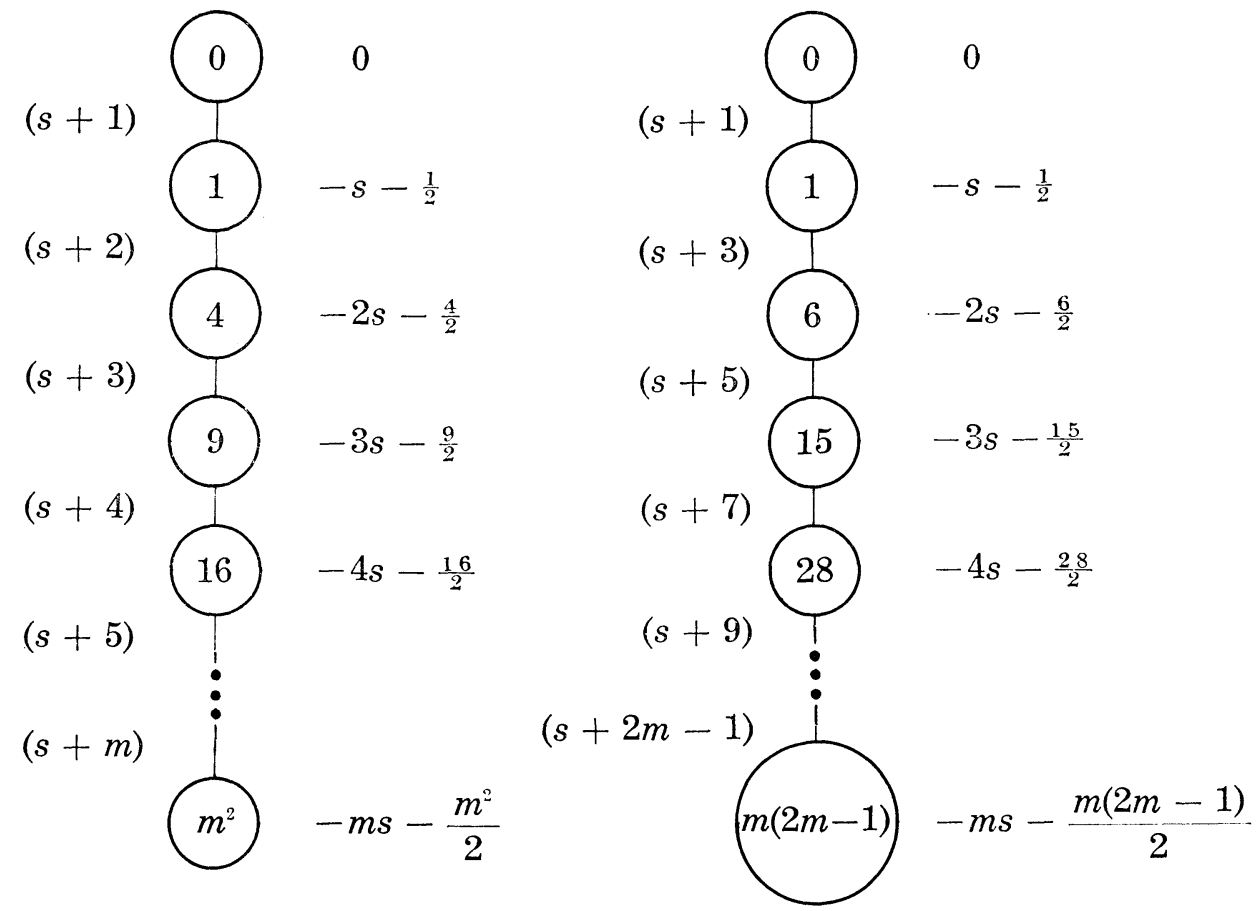

(2) $\ell=2$

Figure 2-4

(3) $\ell=4$ 


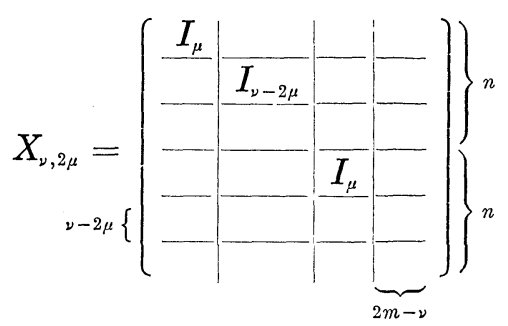

Then $X_{\nu, 2 \mu}$ satisfies the same condition as $X$ and hence there exists an element $g_{1}$ of $S p(n)$ satisfying $g_{1} X=X_{\nu, 2 \mu}$. This implies that $S_{\nu, 2 \mu}=\{X \in V$; $\left.\operatorname{rank} X=\nu, \operatorname{rank}^{t} X J X=2 \mu\right\}(2 m \geqq \nu \geqq 2 \mu \geqq 0)$ consists of a single $G$-orbit, and we complete the orbital decomposition of this space. Put $A \in \mathfrak{B} \mathfrak{p}(n)$ and $D \in \mathfrak{g} \mathfrak{r}(2 m)$ as follows:

$$
\begin{aligned}
& A=\left(\begin{array}{ccc|ccc}
A_{1} & A_{12} & A_{13} & B_{1} & B_{12} & B_{13} \\
A_{21} & A_{2} & A_{23} & { }^{t} B_{12} & B_{2} & B_{23} \\
A_{31} & A_{32} & A_{3} & { }^{t} B_{13} & { }^{t} B_{23} & B_{3} \\
\hline C_{1} & C_{12} & C_{13} & -{ }^{t} A_{1} & -{ }^{t} A_{21} & -{ }^{t} A_{31} \\
{ }^{t} C_{12} & C_{2} & C_{23} & -{ }^{t} A_{12} & -{ }^{t} A_{2} & -{ }^{t} A_{32} \\
\underbrace{t}_{\mu} C_{13} & \underbrace{t}_{\nu-2 \mu} C_{23} & \underbrace{C_{3}}_{n-\nu+\mu} & \underbrace{-t}_{\mu} A_{13} & \underbrace{-t}_{\nu-2 \mu} A_{23} & \underbrace{-{ }^{t} A_{3}}_{n-\nu+\mu}
\end{array}\right], \\
& D=\left(\begin{array}{llll}
D^{2} & D_{12} & D_{13} & D_{14} \\
D_{21} & D_{2} & D_{23} & D_{24} \\
D_{31} & D_{32} & D_{3} & D_{34} \\
D_{41} & \underbrace{D_{42}}_{\nu-2 \mu} & \underbrace{D_{43}}_{\mu} & \underbrace{D_{4}}_{2 m-\nu}
\end{array}\right] \\
& \text { where }{ }^{t} B_{i}=B_{i},{ }^{t} C_{i}=C_{i}(i=1,2,3) .
\end{aligned}
$$

Then, for $\tilde{A}=A \oplus D \in \mathfrak{g}$, we have

$$
\begin{aligned}
d \rho(\tilde{A}) X_{\nu, 2 \mu}= & A X_{\nu, 2 \mu}+X_{\nu, 2 \mu}{ }^{t} D \\
& =\left(\begin{array}{cc|cc}
A_{1}+{ }^{t} D_{1} & A_{12}+{ }^{t} D_{21} & B_{1}+{ }^{t} D_{31}{ }^{t} D_{41} \\
A_{21}+{ }^{t} D_{12} & A_{2}+{ }^{t} D_{2} & { }^{t} B_{12}+{ }^{t} D_{32} & { }^{t} D_{42} \\
A_{31} & A_{32} & { }^{t} B_{13} & 0 \\
\hline C_{1}+{ }^{t} D_{13} & C_{12}+{ }^{t} D_{23} & -{ }^{t} A_{1}+{ }^{t} D_{3} & { }^{t} D_{43} \\
{ }^{t} C_{12} & C_{2} & -{ }^{t} A_{12} & 0 \\
{ }^{t} C_{13} & { }^{t} C_{23} & -{ }^{t} A_{13} & 0
\end{array}\right)
\end{aligned}
$$

and hence the isotropy subalgebra $\mathfrak{g}_{X \nu, 2 \mu}$ is given as follows: 


$$
\begin{aligned}
& \mathrm{g}_{X_{\nu}, 2 \mu}=\left\{\tilde{A}=\left(\begin{array}{ccc|ccc}
A_{1} & 0 & 0 & B_{1} & B_{12} & 0 \\
A_{21} & A_{2} & A_{23} & { }^{t} B_{12} & B_{2} & B_{23} \\
0 & 0 & A_{3} & 0 & { }^{t} B_{23} & B_{3} \\
\hline C_{1} & 0 & 0 & -{ }^{t} A_{1} & -{ }^{t} A_{21} & 0 \\
0 & 0 & 0 & 0 & -{ }^{t} A_{2} & 0 \\
0 & 0 & C_{3} & 0 & -{ }^{t} A_{23} & -{ }^{t} A_{3}
\end{array}\right\}\right. \\
& \left.\oplus\left\{\begin{array}{cc|cc}
-{ }^{t} A_{1} & -{ }^{t} A_{21} & -C_{1} & D_{14} \\
0 & -{ }^{t} A_{2} & 0 & D_{24} \\
\hline-B_{1} & -B_{12} & A_{1} & D_{34} \\
0 & 0 & 0 & D_{4}
\end{array}\right]\right\} \\
& \cong\left\{\begin{array}{c|cc|cc|c}
\frac{A_{2}}{{ }_{0}} & \frac{A_{21}}{A_{1}} & { }^{t} B_{12} & A_{23} & B_{23} & B_{2} \\
\hline 0 & C_{1} & -{ }^{t} A_{1} & & 0 & \begin{array}{r}
B_{12} \\
-{ }^{t} A_{21}
\end{array} \\
\hline 0 & & 0 & A_{3} & B_{3} & { }^{t} B_{23} \\
\hline 0 & & 0 & & 0 & -{ }^{t} A_{2}
\end{array}\right\} \\
& \left.\oplus\left\{\begin{array}{rr|r|r}
A_{1} & B_{1} & -B_{12} & D_{34} \\
C_{1} & -{ }^{t} A_{1} & { }^{t} A_{21} & -D_{14} \\
\hline & 0 & -{ }^{t} A_{2} & D_{24} \\
\hline 0 & 0 & D_{4}
\end{array}\right\}\right\} \\
& \cong(\mathfrak{g} \mathfrak{l}(\nu-2 \mu) \oplus \mathfrak{g} \mathfrak{l}(2 m-\nu) \oplus \mathfrak{S} \mathfrak{p}(\mu) \oplus \mathfrak{S p}(n-\nu+\mu)) \oplus \mathfrak{u}(k)
\end{aligned}
$$

where $\mathfrak{u}(k)$ denotes the Lie algebra of a $k$-dimensional unipotent group with $k=\frac{1}{2}(4 n+1)(\nu-2 \mu)-\frac{3}{2}(\nu-2 \mu)^{2}+\nu(2 m-\nu)$. In this paper, we make a convention that the first (resp. second) $\oplus$ implies the direct sum as Lie algebras (resp. vector spaces) for $\left(\mathfrak{g}_{1} \oplus \mathfrak{g}_{2}\right) \oplus \mathfrak{g}_{3}$.

We identify the dual space $V^{*}$ of $V$ with $V$ by $\langle X, Y\rangle=\operatorname{tr} X^{t} Y$ for $X, Y \in V=M(2 n, 2 m)$, and hence we have $\rho^{*}(g) Y={ }^{t} g_{1}^{-1} Y g_{2}^{-1}$ for $g=\left(g_{1}, g_{2}\right)$ $\in G, \quad Y \in V$ and $d_{\rho^{*}}(\tilde{A}) Y=-{ }^{t} A Y-Y D$ for $\tilde{A}=(A, D) \in \mathrm{g}$. From (3.3), the conormal vector space $V_{\tilde{X}_{\nu, 2},}^{*}$ is given by

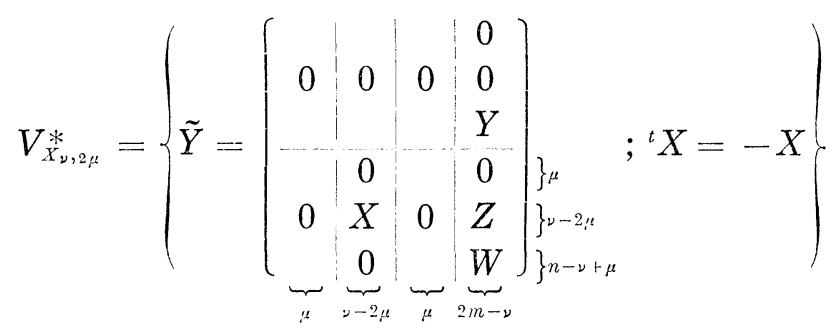




$$
\cong\left\{\tilde{Y}^{\prime}=(\underbrace{\left.\frac{Y_{1}}{0} \mid \frac{Y_{2}}{Y_{3}}\right)_{\}^{\nu-2 \mu}}^{\left.{ }^{2}-\nu+\mu\right)}}_{\nu-2 \mu 2 m-\nu} ;{ }^{t} Y_{1}=-Y_{1}\}\right. \text {. }
$$

Here the isomorphism is obtained by putting $Y_{1}=X, Y_{2}=Z$ and $Y_{3}$ $=\left[\begin{array}{c}Y \\ W\end{array}\right]$. Then the action $d \rho_{X_{\nu}, \mu}$ of $g_{X_{\nu},{ }_{2 \mu}}$ on $V_{X_{\nu, 2 \mu}}^{*}$ is given as follows.

$$
d \rho_{X_{\nu}, \mu}(\tilde{A}) \tilde{Y}^{\prime}=\left(\begin{array}{c|cc}
A_{2} & -B_{23} & A_{23} \\
\hline 0 & -{ }^{t} A_{3} & -C_{3} \\
-B_{3} & A_{3}
\end{array}\right)\left(\begin{array}{c|c}
Y_{1} & \frac{Y_{2}}{2} \\
0 & Y_{3}
\end{array}\right)+\left(\begin{array}{c|c}
Y_{1} & Y_{2} \\
0 & Y_{3}
\end{array}\right)\left(\begin{array}{c|c}
{ }^{t} A_{2} & -D_{24} \\
\hdashline 0 & -D_{4}
\end{array}\right) .
$$

Thus the action on $Y_{1}$-space is isomorphic to $\left(G L(\nu-2 \mu), \Lambda_{2}, V\left(\frac{1}{2}(\nu-2 \mu) \times\right.\right.$ $(\nu-2 \mu-1)))$ and the action on $Y_{3}$-space is isomorphic to $(\operatorname{Sp}(n-\nu+\mu)$ $\left.\times G L(2 m-\nu), \Lambda_{1} \otimes \Lambda_{1}, V(2 n-2 \nu+2 \mu) \otimes V(2 m-\nu)\right)$. First we shall consider the case when $\nu$ is even, i.e., $\nu=2 \nu^{\prime}$. Let $\tilde{Y}_{0}$ be an element of $V_{X_{\nu, 2 \mu}}^{*}$ with $X=\left(\begin{array}{cc}0 & I_{\nu^{\prime}-\mu} \\ -I_{\nu^{\prime}-\mu} & 0\end{array}\right), Y=\left(\begin{array}{cc}I_{m-\nu^{\prime}} & 0 \\ 0 & \end{array}\right), W=\left(\begin{array}{cc}0 & I_{m-\nu} \\ 0\end{array}\right)$ and $Z=0$ in (3.5). Then $\tilde{Y}_{0}$ is a generic point and $\tilde{Y}_{0} \in S_{2 m-2 \mu, 2 m-2 \nu^{\prime}}^{*}$, i.e., $\Lambda_{2 \nu^{\prime}, 2 \mu}=\Lambda_{2 m-2 \mu, 2 m-2 \nu^{\prime}}^{*}$ where $\Lambda_{\nu, 2 \mu}$ (resp. $\Lambda_{\nu, 2 \mu}^{*}$ ) denotes the conormal bundle of $S_{\nu, 2 \mu}$ (resp. $S_{\nu, 2 \mu}^{*}$ ). We shall calculate the order $\operatorname{ord}_{\Lambda_{2 \nu^{\prime}, 2 \mu}} f^{s}$ where $f(X)=P f^{t} X J X$. Let $\tilde{A}_{0}$ be the element of $\mathfrak{g}_{X_{\nu, 2 \mu}}$ with $A_{2}=\frac{1}{2} I_{2\left(\nu^{\prime}-\mu\right)}, D_{4}=-I_{2\left(m-\nu^{\prime}\right)}$, all remaining parts zero in (3.4). Then we have $d \rho\left(\tilde{A}_{0}\right) X_{\nu, 2 \mu}=0$ and $d \rho^{*}\left(\tilde{A}_{0}\right) \tilde{Y}_{0}=\tilde{Y}_{0}$ Since $\delta \chi\left(\tilde{A}_{0}\right)=-\left(2 m-\nu^{\prime}-\mu\right), \operatorname{tr}_{V_{X_{\nu}, 2 \mu}^{*}} \tilde{A}_{0}=\left(\nu^{\prime}-\mu\right)\left(2 \nu^{\prime}-2 \mu-1\right)+4\left(m-\nu^{\prime}\right)(n-$ $\left.2 \nu^{\prime}+\mu\right)+6\left(m-\nu^{\prime}\right)\left(\nu^{\prime}-\mu\right)$ and $\operatorname{dim} V_{X_{\nu}, 2 \mu}^{*}=\left(\nu^{\prime}-\mu\right)\left(2 \nu^{\prime}-2 \mu-1\right)+4(m-$ $\left.\nu^{\prime}\right)\left(n-2 \nu^{\prime}+\mu\right)+4\left(m-\nu^{\prime}\right)\left(\nu^{\prime}-\mu\right)$, we have

$$
\begin{aligned}
\operatorname{ord}_{\Lambda_{2 \nu^{\prime}, 2 \mu}} f^{s}= & -\left(2 m-\nu^{\prime}-\mu\right) s-\frac{1}{2}\left(\nu^{\prime}-\mu\right)\left(2 \nu^{\prime}-2 \mu-1\right) \\
& -2\left(m-\nu^{\prime}\right)\left(n-2 \nu^{\prime}+\mu\right)-4\left(m-\nu^{\prime}\right)\left(\nu^{\prime}-\mu\right) .
\end{aligned}
$$

Let $\tilde{Y}_{1}$ be the element of $V_{X_{\nu}, \mu}^{*}$ with $X=\left(\begin{array}{cc}0 & I_{\nu^{\prime}-\mu} \\ -I_{\nu^{\prime}-\mu} & 0\end{array}\right), Y=\left(\begin{array}{cc}I_{m-\nu^{\prime}+1} & 0 \\ 0 & \end{array}\right)$, $W=\left(\begin{array}{cc}0 & I_{m-\nu^{\prime}-1} \\ 0 & \tilde{Y}_{1}\end{array}\right)$ and $Z=0$. Since $\tilde{Y}_{1}$ is a point of a one-codimensional orbit and $\tilde{Y}_{1} \in S_{2 m-2 \mu, 2\left(m-\nu^{\prime}-1\right)}^{*}$, we have $\Lambda_{2 \nu^{\prime}, 2 \mu} \cap \Lambda_{2\left(\nu^{\prime}+1\right), 2 \mu}=\operatorname{dim} V-1$. They intersect regularly. By Corollary 1-2, we have

$$
b_{\Lambda_{2 \nu^{\prime}, 2 \mu}}(s) / b_{\Lambda_{2\left(\nu^{\prime}+1\right), 2 \mu}}(s)=s+2 n-2 \nu^{\prime} \quad\left(m-1 \geqq \nu^{\prime} \geqq 0\right) .
$$

Now let $\tilde{Y}_{2}$ be the element of $V_{X_{\nu}, 2 \mu}^{*}$ with $X=\left(\begin{array}{cc|c}0 & I_{\nu^{\prime}-\mu-1} & 0 \\ -I_{\nu^{\prime}-\mu-1} & 0 & \\ \hline 0 & & 0\end{array}\right)$, $Y=\left(\begin{array}{rr}I_{m-\nu^{\prime}} & 0 \\ 0 & \end{array}\right), W=\left(\begin{array}{ll}0 & I_{m-\nu^{\prime}} \\ 0\end{array}\right)$ and $Z=0$. Since $\tilde{Y}_{2}$ is a point of the 
other one-codimensional orbit and $\tilde{Y}_{2} \in S_{2(m-\mu-1), 2\left(m-\nu^{\prime}\right)}^{*}$, we have $\operatorname{dim} \Lambda_{2 \nu^{\prime}, 2 \mu} \cap$ $\Lambda_{2 v^{\prime}, 2(\mu+1)}=\operatorname{dim} V-1$. They intersect regularly. By Corollary 1-2, we have

$$
b_{\Lambda_{2 \nu^{\prime}, 2 \mu}}(s) / b_{\Lambda_{2 \nu^{\prime}, 2(\mu+1)}}=s+2 m-2 \mu-1 \quad(m-1 \geq \mu \geq 0) .
$$

Now we shall show that $\Lambda_{\nu, 2 m}$ is not a good holonomic variety when $\nu$ is odd, i.e., $\nu=2 \nu^{\prime}+1$. Let $\tilde{Y}_{0}$ be the element of $V_{X_{\nu, 2 \mu}}^{*}$ with $X=$ $\left(\begin{array}{cc|c}0 & I_{\nu,-\mu} & 0 \\ -I_{\nu,-\mu} & & \\ \hline 0 & 0\end{array}\right), Y=\left(\begin{array}{cc}I_{m-\nu,-1} & 0 \\ 0 & \end{array}\right), W=\left(\begin{array}{cc}0 & I_{m-\nu} \\ & 0\end{array}\right)$ and $Z=0$ in (3.5). Then it is a generic point of the conormal vector space. Let $\widetilde{A}_{0}$ be the element of $\mathrm{g}_{X \nu, 2 \mu}$ with $A_{2}=\left(\begin{array}{cc}\frac{1}{2} I_{2(\nu,-\mu)} & 0 \\ 0 & \beta\end{array}\right), D_{4}=-I_{2 m-\nu}$, all remaining parts zero. Then we have $d \rho\left(\tilde{A}_{0}\right) X_{\nu, 2 \mu}=0$ and $d \rho^{*}\left(\tilde{A}_{0}\right) \tilde{Y}_{0}=\tilde{Y}_{0}$. Therefore, if $\Lambda_{\nu, 2 \mu}$ is a good holonomic variety, $m_{\Lambda_{\nu, 2} \mu^{\prime}}=-\delta \chi\left(\tilde{A}_{0}\right)=2 m-\nu^{\prime}-\mu-1+\beta$ is a non-negative integer which is a contradiction. Thus we obtain the following proposition.

Proposition 3-1. The irreducible regular P.V. $\left(S p(n) \times G L(2 m), \Lambda_{1} \otimes\right.$ $\left.\Lambda_{1}, V(2 n) \otimes V(2 m)\right)(n \geqq 2 m)$ has finitely many orbits $S_{\nu, 2 \mu}=\{X \in M(2 n, 2 m)$; rank $\left.X=\nu, \operatorname{rank}^{t} X J X=2 \mu\right\}(2 m \geqq \nu \geqq 2 \mu \geqq 0)$. When $\nu$ is odd, the conormal bundle $\Lambda_{\nu, 2 \mu}$ of $S_{\nu, 2 \mu}$ is outside $W$, i.e., $\Lambda_{\nu, 2 \mu}$ is not a good holonomic variety. When $\nu$ is even $\left(\nu=2 \nu^{\prime}\right), \Lambda_{\nu, 2 \mu}$ is a good holonomic variety and $\operatorname{ord}_{\Lambda_{\nu, 2 \mu}} f^{s}=-\left(2 m-\nu^{\prime}-\mu\right) s-\frac{1}{2}\left(\nu^{\prime}-\mu\right)\left(2 \nu^{\prime}-2 \mu-1\right)-2\left(m-\nu^{\prime}\right)\left(n-2 \nu^{\prime}\right.$ $+\mu)-4\left(m-\nu^{\prime}\right)\left(\nu^{\prime}-\mu\right)$. We have $\operatorname{dim} \Lambda_{\nu, 2 \mu} \cap \Lambda_{\nu, 2(\mu+1)}=\operatorname{dim} \Lambda_{\nu, 2 \mu} \cap \Lambda_{\nu+2,2 \mu}=$ $\operatorname{dim} V-1$. The b-function $b(s)$ is given by $b(s)=\prod_{k=1}^{m}(s+2 k-1) \cdot \prod_{\ell=0}^{m-1}$ $(s+2 n-2 \ell)$.

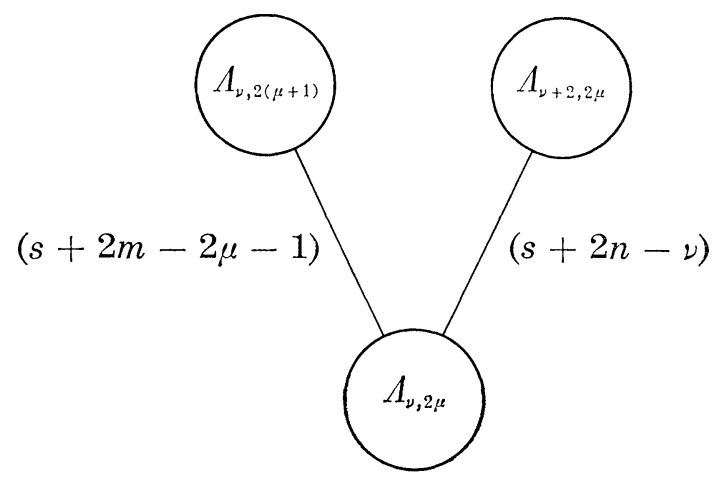

Figure 3-1. ( $($ : even) 


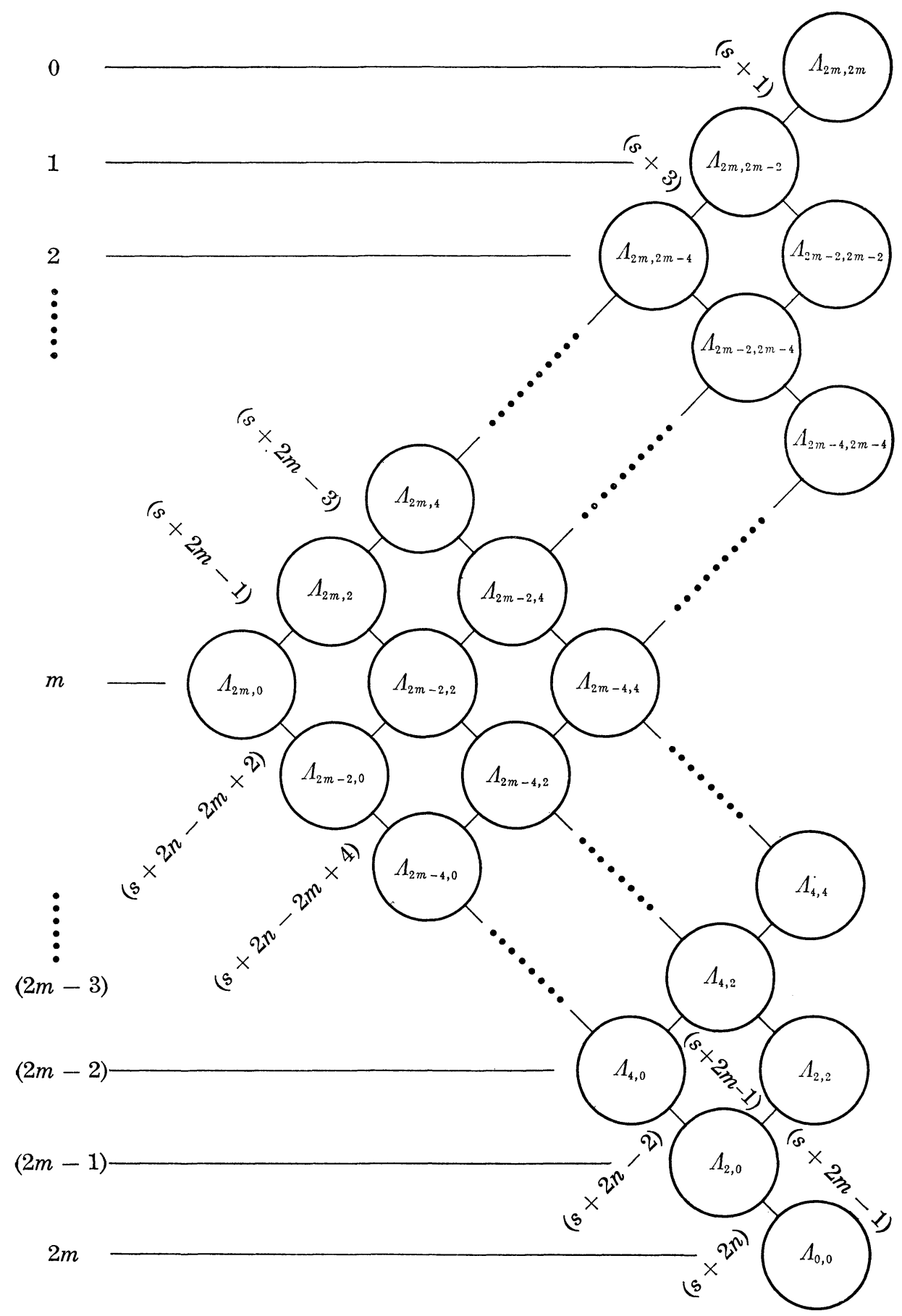

Figure 3-2. Holonomy diagram of $\left(S p(n) \times G L(2 m), \Lambda_{1} \otimes A_{1}, V(2 n) \otimes V(2 m)\right)$ with $n \geqq 2 m$. 


\section{§4. $\left(\operatorname{Spin}(10) \times G L(2)\right.$, half-spin rep. $\left.\otimes \Lambda_{1}, V(16) \otimes V(2)\right)$}

The representation space $V(16) \otimes V(2)$ is identified with $V=V(16) \oplus$ $V(16)$ where $V(16)$ is spanned by $1, e_{i} e_{j}, e_{k} e_{\ell} e_{m} e_{n}(1 \leq i<j \leq 5,1 \leq k<\ell$ $<m<n \leq 5$ ) (See p. 110-112 in [1]). The action $\rho=\rho_{1} \otimes \Lambda_{1}$ is given by $\rho(g) x=\left(\rho_{1}\left(g_{1}\right) X, \rho_{1}\left(g_{1}\right) Y\right)^{t} g_{2}$ for $g=\left(g_{1}, g_{2}\right) \in \operatorname{Spin}(10) \times G L(2), x=(X, Y) \in V$ $=V(16) \oplus V(16)$ where $\rho_{1}$ denotes the even half-spin representation of $\operatorname{Spin}(10)$ on $V(16)$. First of all, we shall complete the orbital decomposition of this space. J-I. Igusa completed the orbital decomposition of (Spin(10), $\left.\rho_{1}, V(16)\right)$ (See [3]). There exist three orbits $S_{m}^{\prime}=\rho_{1}(\operatorname{Spin}(10)) \cdot x_{m}^{\prime}(m=0,5$, 16) where $S_{m}^{\prime}$ denotes the $m$-codimensional $S p i n(10)$-orbit and $x_{0}^{\prime}=1+$ $e_{1} e_{2} e_{3} e_{4}, x_{5}^{\prime}=1, x_{16}^{\prime}=0$. If $\lambda \in C^{\times}$, for any index $i$ satisfying $1 \leq i \leq 5$, we put $S_{i}(\lambda)=\lambda^{-1}+\left(\lambda-\lambda^{-1}\right) e_{i} f_{i}$. Then $S_{i}(\lambda)$ is an element of $\operatorname{Spin}(10)$. For any two distinct indices $i, j$ satisfying $1 \leq i, j \leq 10, j \neq i+5, i \neq j+5$, we put $S_{i j}(\lambda)=1+\lambda e_{i} e_{j}=\exp \left(\lambda e_{i} e_{j}\right)$ where $e_{k}=f_{k-5}$ for $6 \leq k \leq 10$ (See [1], [3]). Then $S_{i j}(\lambda)$ is an element of $\operatorname{Spin}(10)$ satisfying $S_{i j}(\lambda) S_{j i}(\lambda)=1$.

Proposition 4-1. The triplet $\left(\operatorname{Spin}(10) \times G L(2)\right.$, half-spin rep. $\otimes A_{1}$, $V(16) \otimes V(2))$ has nine orbits $S_{m}=\rho(G) x_{m} \quad(m=0,1,4,8,9,13,15,20,32)$ where $S_{m}$ denotes the m-codimensional orbit.

$$
\begin{aligned}
& \text { (1) } x_{0}=\left(1+e_{1} e_{2} e_{3} e_{4}, e_{1} e_{5}+e_{2} e_{3} e_{4} e_{5}\right) \\
& \text { (2) } x_{1}=\left(1+e_{1} e_{2} e_{3} e_{4}, e_{1} e_{2}+e_{2} e_{3} e_{4} e_{5}\right) \\
& \text { (3) } x_{4}=\left(1, e_{1} e_{5}+e_{2} e_{3} e_{4} e_{5}\right) \\
& \text { (4) } x_{8}=\left(1, e_{1} e_{2} e_{3} e_{4}\right) \\
& \text { (5) } x_{9}=\left(1, e_{1} e_{2}+e_{3} e_{4}\right) \\
& \text { (6) } x_{13}=\left(1, e_{1} e_{2}\right) \\
& \text { (7) } x_{15}=\left(1+e_{1} e_{2} e_{3} e_{4}, 0\right) \\
& \text { (8) } x_{20}=(1,0) \\
& \text { (9) } x_{32}=(0,0)
\end{aligned}
$$

Proof. Let $\tilde{x}=(x, y)$ be a representative of one of the orbits of $V=$ $V(16) \oplus V(16)$. Then we may assume that $x=0$, 1 , or $1+e_{1} e_{2} e_{3} e_{4}$ by the action of $\operatorname{Spin}(10)$. If $x=0$, then we have also $y=1+e_{1} e_{2} e_{3} e_{4}, 1,0$, i.e., (7), (8), (9) respectively. Note that we can exchange $x$ and $y$ in $\tilde{x}=(x, y)$ by the action of $G L(2)$. Assume that $x=1$. We may put $y=y_{0}+y_{2}+y_{4}$ $\neq 0$ where $y_{0}=y_{0} \cdot 1, y_{2}=\sum y_{i j} e_{i} e_{j}$ and $y_{4}=\sum y_{r s t u} e_{r} e_{s} e_{t} e_{u}$. We may assume that $y_{0}=0$ by the action of $\left(\begin{array}{cc}1 & 0 \\ -y_{0} & 1\end{array}\right)$. If $y=y_{2} \neq 0$, we may assume that $y_{12}=1$ by the action of some $S_{i j}(\lambda)(i=1,2 ; j \geq 6)$ and $\left(\begin{array}{ll}1 & 0 \\ 0 & \lambda\end{array}\right)$ if necessary. 
In this case, we have $y=e_{1} e_{2}+y_{34} e_{3} e_{4}+y_{35} e_{3} e_{5}+y_{45} e_{4} e_{5}$ by $S_{j 7}\left(-y_{1 j}\right)$ and $S_{j 6}\left(y_{2 j}\right)$ for $j=3,4,5$. If $y_{34}=y_{35}=y_{45}=0$, we have (6), and otherwise we may assume that $y_{34}=1, y_{35}=y_{45}=0$ by the action of suitable elements of $\left\{S_{3,10}(\lambda), S_{4,10}(\lambda), S_{58}(\lambda), S_{59}(\lambda) ; \lambda \in C\right\}$, i.e., (5). If $y_{4} \neq 0$, we may assume that $y_{4}=e_{1} e_{2} e_{3} e_{4}$. By the action of $S_{89}\left(y_{12}\right)$ and $\left(\begin{array}{cc}1 & 0 \\ y_{12} y_{34} & 1\end{array}\right)$, we have $y_{12}=0$. Similarly $y_{i j}=0$ for $1 \leq i<j \leq 4$, and hence $y=\sum_{j=1}^{4} y_{j 5} e_{j} e_{5}+e_{1} e_{2} e_{3} e_{4}$. If $y_{j 5}=0$ for all $j=1, \cdots, 4$, we have (4). In the other case, we may assume that $y_{15}=1$ and $y_{j 5}=0 \quad(2 \leq j \leq 4)$. By the action of $S_{56}(-1)$ and $S_{1,10}(1)$, we have (3).

Finally assume that $x=1+e_{1} e_{2} e_{3} e_{4}$. We may put $y=y_{2}+y_{4}$. If $y_{4}$ $\neq 0$, we may assume that $y_{4}=e_{2} e_{3} e_{4} e_{5}$ or $y_{4}=e_{1} e_{2} e_{3} e_{4}$. In the former case, if $y_{15} \neq 0$, we may assume that $y_{15}=1$ by the action of $S_{1}(\lambda) S_{5}(\lambda) S_{2}\left(\lambda^{-1}\right)$ and $\lambda I_{2}$ where $\lambda^{4} \cdot y_{15}=1$. Then by the action of $S_{j 10}\left(-y_{1 j}\right), S_{j 6}\left(-y_{j 5}\right)(j=2$, $3,4), S_{9,10}\left(y_{23}\right), S_{8,10}\left(-y_{24}\right)$ and $S_{7,10}\left(y_{34}\right)$, we have (1). If $y_{15}=0$, we may assume that $y_{35}=y_{45}=0$ by $\left\{S_{28}(\lambda), S_{29}(\lambda), S_{37}(\lambda), S_{47}(\lambda) ; \lambda \in C\right\}$. Then by $S_{8,10}\left(-y_{24}\right)$ and $S_{9,10}\left(y_{23}\right)$, we may assume that $y_{24}=y_{23}=0$. By some $S_{39}(\lambda)$ and $S_{28}(\lambda)$, we may also assume that $y_{14}=0$, i.e., $y=y_{12} e_{1} e_{2}+y_{13} e_{1} e_{3}+y_{34} e_{3} e_{4}$ $y_{25} e_{2} e_{5}+e_{2} e_{3} e_{4} e_{5}$. By the action of $S_{7,10}\left(y_{34}\right),\left(\begin{array}{cc}1 & 0 \\ y_{25} y_{34} & 1\end{array}\right)$ and $S_{1,10}\left(y_{25} y_{34}\right)$, we have $y_{34}=0$. By $S_{89}\left(y_{25}\right)$ and $S_{12}\left(y_{25}\right)$, we also have $y_{25}=0$, i.e., $y=y_{12} e_{1} e_{2}$ $+y_{13} e_{1} e_{3}+e_{2} e_{3} e_{4} e_{5}$, where we may assume that $y_{13}=0$. If $y_{12} \neq 0$, we have (2). If $y_{12}=0$, it is transferred to $x_{4}$ by $S_{12}(-1), S_{89}(-1), S_{34}(-1), S_{67}(-1)$, $S_{17}(1), S_{26}(-1), S_{56}(-1), S_{1,10}(1),\left(\begin{array}{ll}1 & 0 \\ 1 & 1\end{array}\right), S_{6,10}(1), S_{15}(1)$ and $\left(\begin{array}{rr}1 & -1 \\ 0 & 1\end{array}\right)$.

Now consider the latter case, i.e., $y_{4}=e_{1} e_{2} e_{3} e_{4}$. If some of $y_{j 5}(1 \leq j \leq 4)$ is not zero, we may assume that $y=e_{1} e_{5}+y_{23} e_{2} e_{3}+e_{1} e_{2} e_{3} e_{4}$. If $y_{23}=0$, it is transferred to $x_{4}$ by $S_{15}(1),\left(\begin{array}{rr}1 & -1 \\ 0 & 1\end{array}\right), S_{56}(-1)$ and $S_{1,10}(1)$. If $y_{23} \neq 0$, we may assume that $y_{23}=1$. In this case, it is transferred to $x_{1}$ by $S_{69}(1)$, $S_{23}(1), S_{4,10}(-1), S_{78}(1), S_{14}(1), S_{46}(1), S_{19}(-1), S_{29}(-1), S_{47}(1)$. When all $y_{j 5}=0$ for $1 \leq j \leq 4, y=\sum_{1 \leq i<j \leq 4} y_{i j} e_{i} e_{j}+e_{1} e_{2} e_{3} e_{4}$. If all $y_{i j}=0$ for $1 \leq i<j \leq 4$, it is transferred to $x_{8}$ by $\left(\begin{array}{rr}1 & -1 \\ 0 & 1\end{array}\right)$. In the other case, we may assume that $y=e_{1} e_{2}+y_{34} e_{3} e_{4}+e_{1} e_{2} e_{3} e_{4}$. By the action of $S_{67}(\lambda), S_{34}(\lambda)$ and $\left(\begin{array}{ll}1 & 0 \\ \lambda & 1\end{array}\right)$ with $\lambda^{2}-\lambda-y_{34}=0$, we have $y=e_{1} e_{2}+(1+2 \lambda) e_{1} e_{2} e_{3} e_{4}$. If $(1+2 \lambda) \neq 0$, it is transferred to $x_{8}$ by $S_{89}(\mu), S_{12}(\mu)$ and $\left(\begin{array}{rr}1 & -\mu \\ 0 & \mu\end{array}\right)$ with $\mu=\frac{1}{1+2 \lambda}$. If $(1+2 \lambda)=0$, it is equivalent to $x_{9}$ by $S_{67}(-1), S_{12}(-1),\left(\begin{array}{rr}0 & 1 \\ 1 & -1\end{array}\right), S_{1}(\sqrt{-1})$, and $\sqrt{-1} I_{2}$. 
Finally consider the case $y=y_{2}$, i.e., $y_{4}=0$. Since $y \neq 0$, we may assume that $y=e_{1} e_{2}+y_{34} e_{3} e_{4}+\sum_{j=1}^{4} y_{j 5} e_{j} e_{5}$. If $y_{34}=y_{j 5}=0$ for $1 \leq j \leq 4$, it is equivalent to $x_{9}$ as we have already seen. If $y_{34} \neq 0$ and $y_{j 5}=0$ for $1 \leq j \leq 4$, it is transferred to $x_{8}$ by $S_{34}(\lambda),\left(\begin{array}{cc}1 & -1 / \lambda \\ 1 & 0\end{array}\right), S_{12}(1 / \lambda),\left(\begin{array}{cc}1 & 0 \\ 0 & 1 / 2 \lambda\end{array}\right)$, $S_{67}(\lambda / 2), S_{89}(1 / 2 \lambda),\left(\begin{array}{cc}1 & 0 \\ 1 / 4 & 1\end{array}\right)$ with $\lambda^{2}=y_{34}$. If some of $y_{j 5}(1 \leq j \leq 4)$ is not zero, $y$ is equivalent to an element of the form $e_{1} e_{5}+y_{23} e_{2} e_{3}+y_{24} e_{2} e_{4}+y_{34} e_{3} e_{4}$. If $y_{i j}=0(2 \leq i<j \leq 4)$, it is equivalent to $x_{4}$ as we have already seen. In the other case, we have $y=e_{1} e_{5}+e_{3} e_{4}$. By the action of $S_{26}(-1), S_{17}(1)$, $S_{34}(-1), S_{67}(-1), S_{7,10}(1),\left(\begin{array}{rr}1 & 0 \\ -1 & 1\end{array}\right), S_{89}(-1), S_{12}(-1), S_{1,10}(-1)$, it is equivalent to $x_{1}$. About the codimension of these orbits, we will see later. Q.E.D.

By the degree formula (See Proposition 15, $\$ 4$ in [1]), we know that there exists a relatively invariant irreducible polynomial $f(x, y)$ of degree four which is unique up to a constant multiple. We shall give an explicit form of $f(x, y)$ after $H$. Kawahara's work (Master Thesis in Japanese, University of Tokyo, 1974).

For an element $x=x_{0}+\sum_{i<j} x_{i j} e_{i} e_{j}+\sum_{k} x_{k}^{*} e_{k}^{*}$ of $V(16)$ where $e_{k} e_{k}^{*}$ $=e_{1} e_{2} e_{3} e_{4} e_{5}$ for $1 \leq k \leq 5$, let $X=\left(x_{i j}\right)$ be the skew-symmetric matrix of degree five determined by $x_{i j}$, and $X_{i}$ the skew-symmetric matrix of degree four obtained from $(-1)^{i} X$ by crossing out its $i$-th line and column $(1 \leq$ $i \leq 5)$. We denote by $\operatorname{Pf}(Y)$ the Pfaffian of the skew-symmetric matrix $Y=\left(y_{i j}\right)$ of degree four, i.e., $\operatorname{Pf}(Y)=y_{12} y_{34}-y_{13} y_{24}+y_{14} y_{23}$. We define ten quadratic forms $Q_{i}(x)$ on $V(16)$ by $Q_{i}(x)=\sum_{j=1}^{5} x_{i j} x_{j}^{*}$ and $Q_{i+5}(x)=x_{0} x_{i}^{*}+$ $\operatorname{Pf}\left(X_{i}\right)$ for $1 \leq i \leq 5$.

Proposition 4-2 (H. Kawahara).

(1) $\rho_{1}(\operatorname{Spin}(10)) \cdot 1=\left\{x \in V(16) ; Q_{i}(x)=0(1 \leq i \leq 10)\right\}-\{0\}$, where $\rho_{1}$ denotes the even half-spin representation. Moreover, this is the totality of pure spinors.

(2) The relative invariant $f(x, y)$ of $\left(\operatorname{Spin}(10) \times G L(2), \rho_{1} \otimes \Lambda_{1}, V(16) \oplus\right.$ $V(16))$ is given by $f(x, y)=\sum_{i=1}^{5} B_{i}(x, y) B_{i+5}(x, y)$ for $(x, y) \in V(16) \oplus V(16)$ where $B_{i}(x, y)=Q_{i}(x+y)-Q_{i}(x)-Q_{i}(y)$ is the associated bilinear form of $Q_{i}(x)$ for $1 \leq i \leq 10$.

Proof. We shall use the same notation as in [4]. By simple calculation, we have $\beta_{1}(x, x)=(1 / 8) \sum_{i=1}^{10} Q_{i}(x) e_{i}$. Since $\beta_{1}\left(\rho_{1}(s) x, \rho_{1}(s) x\right)=\lambda(s)$. $\zeta_{1}(\chi(s)) \cdot \beta_{1}(x, x)$ for $s \in \operatorname{Spin}(10)$ where $\zeta_{1}$ is the representation $A_{1}$ of $S O(10)$ $=\chi(\operatorname{Spin}(10))($ See p. 90 in [4]), we have 


$$
\sum_{i=1}^{10} Q_{i}\left(\rho_{1}(s) x\right) e_{i}=\lambda(s) \cdot \zeta_{1}(\chi(s)) \cdot \sum_{i=1}^{10} Q_{i}(x) e_{i} .
$$

This implies that $W=\left\{x \in V(16) ; Q_{i}(x)=0,1 \leq i \leq 10\right\}$ is a $\operatorname{Spin}(10)$-invariant subspace. From the orbital decomposition, it is clear that $W=S_{5}^{\prime} \cup$ $S_{16}^{\prime}$, i.e., $S_{5}^{\prime}=W-\{0\}$. Since the totality of pure spinors in $V(16)$ is a single $\Gamma^{+}$-orbit where $\Gamma^{+}$denotes the even Clifford group, and $\beta_{1}(x, x)=0$ for a pure spinor $x$ (See [4]), we have (1). From (4.1), $F(x)=\sum_{i=1}^{5} Q_{i}(x)$ $Q_{i+5}(x)$ is invariant under the action $\rho_{1}$ of $\operatorname{Spin}(10)$ since $\tilde{f}(y)=\sum_{l=1}^{5} y_{i} y_{i+5}$ for $y=\sum_{l=1}^{10} y_{i} e_{i}$ is invariant under the action $\zeta_{1}$ of $S O(10)=\chi(\operatorname{Spin}(10))$. The triplet (Spin(10), $\left.\rho_{1}, V(10)\right)$ has no relative invariant (See [1]) and hence we have $F(x) \equiv 0$. By using (4.1), it is clear that $f(x, y)$ is invariant under the action of $\operatorname{Spin}(10)$. We shall show that $f(x, y)$ is relatively invariant under $G L(2)$. Assume that $Q_{i}(x)$ (resp. $Q_{i_{+5}}(x)$ ) has a term $x_{i_{1}} x_{i_{2}}$ (resp. $\left.x_{i_{3}} x_{i_{4}}\right)(1 \leq i \leq 5)$. Since $F(x) \equiv 0$, we may assume that $Q_{j}(x)$ (resp. $\left.Q_{j+5}(x)\right)$ has a term $x_{i_{1}} x_{i_{3}}$ (resp. $x_{i_{2}} x_{i_{4}}$ ) for some $j$ satisfying $1 \leq j \leq 5$. This implies that $f(x, y)=\sum_{i=1}^{5} B_{i}(x, y) B_{i+5}(x, y)$ is a linear combination of terms of the following form:

$$
\begin{aligned}
& \left(x_{i_{1}} y_{i_{2}}+y_{i_{1}} x_{i_{2}}\right)\left(x_{i_{3}} y_{i_{4}}+y_{i_{3}} x_{i_{4}}\right)-\left(x_{i_{1}} y_{i_{3}}+y_{i_{1}} x_{i_{3}}\right)\left(x_{i_{2}} y_{i_{4}}+y_{i_{2}} x_{i_{4}}\right) \\
& \quad=\operatorname{det}\left(\begin{array}{ll}
x_{i_{2}} & y_{i_{2}} \\
x_{i_{3}} & y_{i_{3}}
\end{array}\right) \cdot \operatorname{det}\left(\begin{array}{ll}
x_{i_{4}} & y_{i_{4}} \\
x_{i_{1}} & y_{i_{1}}
\end{array}\right) .
\end{aligned}
$$

Hence it is clear that $f(x, y)$ is relatively invariant under $G L(2)$. Since $f\left(1+e_{1} e_{2} e_{3} e_{4}, e_{1} e_{5}+e_{2} e_{3} e_{4} e_{5}\right)=1$, it is not identically zero.

Q.E.D.

Now we shall consider the micro-differential equation $\mathfrak{M}=\mathscr{E} f(x, y)^{s}$ and by constructing its holonomy diagram, we shall calculate the $b$-function of this space.

Since $G=\operatorname{Spin}(10) \times G L(2)$ is reductive, we have $\left(G, \rho^{*}, V^{*}\right) \cong(G, \rho, V)$ and hence the dual space $V^{*}$ has also nine $G$-orbits $S_{m}^{*}(m=0,1,4,8,9,13$, $15,20,32)$. We identify $V$ and $V^{*}$ by taking $\left(e_{i_{1}} \cdots e_{2_{k}}, e_{j_{1}} \cdots e_{j_{6}}\right)(k, \ell=0$, $2,4)$ as a dual basis, where $e_{i_{1}} \cdots e_{i_{k}}=1$ for $k=0$. We denote by $\Lambda_{m}$ (resp. $\Lambda_{m}^{*}$ ) the conormal bundle of $S_{m}$ (resp. $S_{m}^{*}$ ).

(1) The isotropy subalgebra $g_{x_{0}}$ at $x_{0}=\left(1+e_{1} e_{2} e_{3} e_{4}, e_{1} e_{5}+e_{2} e_{3} e_{4} e_{5}\right)$ is isomorphic to $\left(g_{2}\right) \oplus \mathfrak{g l}(2)$ (See (5.40) and (5.42) in [1]). Since $\Lambda_{0}=V \times\{0\}$ $=\Lambda_{33}^{*}, \Lambda_{0}$ is a good holonomic variety and we have $\operatorname{ord}_{\Lambda_{0}} f^{s}=0$.

(2) The isotropy subalgebra $\mathfrak{g}_{x_{1}}$ at $x_{1}=\left(1+e_{1} e_{2} e_{3} e_{4}, e_{1} e_{2}+e_{2} e_{3} e_{4} e_{5}\right)$ is isomorphic to $(\mathfrak{g r}(1) \oplus \mathfrak{g r}(2) \oplus \mathfrak{g l}(2)) \oplus \mathfrak{u}(11)$ (See (5.43) in [1]). The conormal vector space $V_{x_{1}}^{*}$ is spanned by $\left(e_{1} e_{3} e_{4} e_{5},-e_{1} e_{5}\right)=y_{1} \in S_{13}^{*}$. Hence $\Lambda_{1}=$ 
$\overline{G\left(x_{1}, y_{1}\right)}=\Lambda_{13}^{*}$ and $\Lambda_{13}=\Lambda_{1}^{*}$. Let $A_{0}$ be an element of $g_{x_{1}}$ with $d_{11}=d_{22}=$ $-1 / 4$, all remaining parts zero in (5.43) of [1]. Then we have $d \rho\left(A_{0}\right) x_{1}=0$ and $d \rho^{*}\left(A_{0}\right) y_{1}=y_{1}$. Since $\delta \chi\left(A_{0}\right)=2\left(d_{11}+d_{22}\right)=-1, \operatorname{tr}_{V_{x_{1}}^{*}} A_{0}=\operatorname{dim} V_{x_{1}}^{*}=1$, we have $\operatorname{ord}_{\Lambda_{1}} f^{s}=-s-1 / 2$. It is clear that $\Lambda_{0}$ and $\Lambda_{1}$ intersect regularly and $G_{0}$-prehomogeneously with codimension one. Hence we have $b_{\Lambda_{1}}(s) / b_{\Lambda_{0}}(s)$ $=(s+1)$. Note that $G_{0}=\{g \in G ; \chi(g)=1\}$.

(3) The isotropy subalgebra $g_{x_{4}}$ at $x_{4}=\left(1, e_{1} e_{5}+e_{2} e_{3} e_{4} e_{5}\right)$ is, by simple calculation using (5.38) in [1], given as follows:

$$
\begin{aligned}
\mathfrak{g}_{x_{4}}= & \left\{\tilde{A}=\left(\frac{A}{C} \mid \frac{0}{-{ }^{t} A}\right) \oplus\left(\begin{array}{cc|c}
3 \varepsilon-\eta & 0 \\
c & \eta
\end{array}\right) ; A=\left(\begin{array}{c|c|c}
3 \varepsilon & 0 & 0 \\
\hline * & \frac{\varepsilon I_{3}+X}{*} & 0 \\
\hline * 2 n
\end{array}\right),\right. \\
& \left.X \in \mathfrak{B I}_{(}(3),{ }^{t} C=-C \quad \text { with } \quad c_{i 5}=0, i=1, \cdots, 4\right\}
\end{aligned}
$$

Put $\omega_{1}=\left(e_{1} e_{3} e_{4} e_{5}, 0\right), \omega_{2}=\left(-e_{1} e_{2} e_{4} e_{5}, 0\right), \omega_{3}=\left(e_{1} e_{2} e_{3} e_{5}, 0\right)$, and $\omega_{4}=\left(e_{1} e_{2} e_{3} e_{4}, 0\right)$. Then the conormal vector space $V_{x_{4}}^{*}$ is spanned by $\omega_{1}, \cdots, \omega_{4}$. The action $d \rho_{x_{4}}$ of $g_{x_{4}}$ on $V_{x_{4}}^{*}$ is given as follows:

$$
d \rho_{x_{4}}(\tilde{A})\left(\omega_{1}, \cdots, \omega_{4}\right)=\left(\omega_{1}, \cdots, \omega_{4}\right)\left(\begin{array}{c|c}
(2 \eta-5 \varepsilon) I_{3}+X & 0 \\
* * 2 & * 6 \varepsilon
\end{array}\right.
$$

Since $\omega_{1}$ is a generic point, we have $\Lambda_{4}=\Lambda_{20}^{*}$ and $\Lambda_{20}=\Lambda_{4}^{*}$. Let $A_{0}$ be an element of $g_{x_{4}}$ with $2 \eta-5 \varepsilon=1$, all remaining parts zero except $\varepsilon$ and $\eta$ in (4.3). Then $d \rho\left(A_{0}\right) x_{4}=0$ and $d \rho^{*}\left(A_{0}\right) \omega_{1}=\omega_{1}$. However we have $\delta \chi\left(A_{0}\right)=6 \varepsilon$ which is not definite. If $\Lambda_{4}$ is a good holonomic variety, this must be definite by Proposition $1-3$, and hence $\Lambda_{4}$ is not a good holonomic variety, i.e., $\Lambda_{4} \not \subset W$. Note that the P.V. $\left(G_{x_{4}}, \rho_{x_{4}}, V_{x_{4}}^{*}\right)$ has no relative invariant.

(4) The isotropy subalgebra $\mathfrak{g}_{x_{8}}$ at $x_{8}=\left(1, e_{1} e_{2} e_{3} e_{4}\right)$ is given as follows:

$$
\begin{aligned}
\mathfrak{g}_{r_{8}}= & \left\{\tilde{X}=\left(\begin{array}{c|c|c|c}
\varepsilon I_{4}+X & \gamma & 0 & 0 \\
\hline 0 & 2 \eta & 0 & 0 \\
\hline 0 & \delta & -\varepsilon I_{4}-{ }^{t} X & 0 \\
\hline-{ }^{t} \delta & 0 & -{ }^{t} \gamma & -2 \eta
\end{array}\right] \oplus\left(\begin{array}{cc}
\eta+2 \varepsilon & 0 \\
0 & \eta-2 \varepsilon
\end{array}\right) ;\right. \\
& \left.X \in \mathfrak{g l}(4), \gamma, \delta \in C^{4}\right\} \cong(\mathfrak{g l}(1) \oplus \mathfrak{g l}(1) \oplus \mathfrak{g l}(4)) \oplus \mathfrak{u}(8) .
\end{aligned}
$$


Put $\omega_{1}=\left(e_{2} e_{3} e_{4} e_{5}, 0\right), \omega_{2}=-\left(e_{1} e_{3} e_{4} e_{5}, 0\right), \omega_{3}=\left(e_{1} e_{2} e_{4} e_{5}, 0\right), \omega_{4}=-\left(e_{1} e_{2} e_{3} e_{5}\right.$, $0), \omega_{5}=\left(0, e_{1} e_{5}\right), \omega_{6}=\left(0, e_{2} e_{5}\right), \omega_{7}=\left(0, e_{3} e_{5}\right), \omega_{8}=\left(0, e_{4} e_{5}\right)$. Then the conormal vector space $V_{x_{8}}^{*}$ is spanned by $\omega_{1}, \cdots, \omega_{8}$, and the action $d \rho_{x_{8}}$ of $\mathfrak{g}_{x_{8}}$ on $V_{x_{8}}^{*}$ is given as follows.

$$
d \rho_{x_{8}}(\tilde{X})\left(\omega_{1}, \cdots, \omega_{8}\right)=\left(\omega_{1}, \cdots, \omega_{8}\right)\left(\frac{(3 \varepsilon-2 \eta) I_{4}+X}{0} \mid \frac{0}{-(3 \varepsilon+2 \eta) I_{4}-{ }^{t} X}\right)
$$

where $\tilde{X} \in \mathfrak{g}_{x_{8}}$ in (4.5).

Any relative invariant of $\left(G_{x_{8}}, \rho_{x_{8}}, V_{x_{8}}^{*}\right)$ is of the form $c \cdot g(x)^{m}(c \in C$, $m \in Z$ ) where $g(x)=\sum_{i=1}^{4} x_{i} x_{i+4}$ for $x=\sum_{i=1}^{8} x_{i} \omega_{i}$. Clearly $y_{8}=\omega_{1}+\omega_{5}=$ $\left(e_{2} e_{3} e_{4} e_{5}, e_{1} e_{5}\right)$ is a generic point, and $y_{8}^{\prime}=\omega_{1}+\omega_{6}=\left(e_{2} e_{3} e_{4} e_{5}, e_{2} e_{5}\right)$ is a point of the one-codimensional orbit. Hence we have $\Lambda_{8}=\Lambda_{8}^{*}$ and $\operatorname{dim} \Lambda_{1} \cap \Lambda_{8}$ $=\operatorname{dim} V-1$. Since $\Lambda_{13}=\Lambda_{1}^{*}$, we have also $\operatorname{dim} \Lambda_{8} \cap \Lambda_{13}=\operatorname{dim} V-1$. Note that $\left(G_{x_{8}}, \rho_{x_{8}}, V_{x_{8}}^{*}\right)$ is a regular P.V. since $\rho_{x_{8}}\left(G_{x_{8}}\right)$ and its generic isotropy subgroup are reductive (See [1]). By Corollary 1-7, $\Lambda_{8}$ is a good holonomic variety. Let $\tilde{X}_{0}$ be an element of $\mathfrak{g}_{x_{8}}$ with $\eta=-\frac{1}{2}$, all remaining parts zero in (4.5). Then $d \rho\left(\tilde{X}_{0}\right) x_{8}=0$ and $d \rho^{*}\left(\tilde{X}_{0}\right) y_{8}=y_{8}$. Since $\delta \chi\left(\tilde{X}_{0}\right)=4 \eta=$ $-2, \operatorname{tr}_{V_{x_{8}}^{*}} \tilde{X}_{0}=-16 \eta=8$ and $\operatorname{dim} V_{x_{8}}^{*}=8$, we have $\operatorname{ord}_{\Lambda_{8}} f^{s}=-2 s-\frac{8}{2}$. Since $m_{A_{8}}-m_{A_{1}}=1$, they intersect regularly. By Corollary 1-2, we have $b_{\Lambda_{8}}(s) / b_{\Lambda_{1}}(s)=(s+4)$.

(5) We shall calculate the isotropy subalgebra at $x_{9}^{\prime}=\left(1, e_{1} e_{3}+e_{2} e_{4}\right)$ instead of $x_{9}=\left(1, e_{1} e_{2}+e_{3} e_{4}\right)$. It is given as follows.

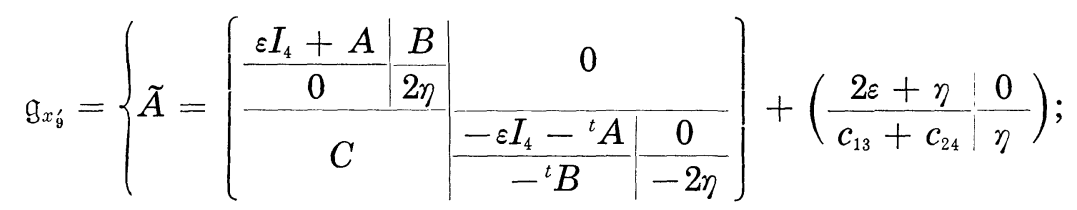

$$
\begin{aligned}
& \left.A \in \mathfrak{g} \mathfrak{p}(2), B \in C^{4}, C=-{ }^{t} C=\left(c_{i j}\right)\right\} \cong(\mathfrak{g r}(1) \oplus \mathfrak{g r}(1) \oplus \mathfrak{g h}(2)) \oplus \mathfrak{u}(4) \text {. }
\end{aligned}
$$

Put $\omega_{1}=\left(e_{2} e_{3} e_{4} e_{5}, 0\right), \omega_{2}=-\left(e_{1} e_{3} e_{4} e_{5}, 0\right), \omega_{3}=\left(e_{1} e_{2} e_{4} e_{5}, 0\right), \omega_{4}=-\left(e_{1} e_{2} e_{3} e_{5}, 0\right)$, $\omega_{5}=\left(e_{1} e_{2} e_{3} e_{4}, 0\right), \omega_{6}=-\left(e_{3} e_{5}, e_{2} e_{3} e_{4} e_{5}\right), \omega_{7}=\left(-e_{4} e_{5}, e_{1} e_{3} e_{4} e_{5}\right), \omega_{8}=\left(e_{1} e_{5},-e_{1} e_{2} e_{4} e_{5}\right)$, $\omega_{9}=\left(e_{2} e_{5}, e_{1} e_{2} e_{3} e_{5}\right)$. Then the conormal vector space $V_{x^{\prime}}^{*}$ is spanned by these $\omega_{1}, \cdots, \omega_{9}$ and the action $d \rho_{x_{g^{\prime}}}$ of $g_{x_{g^{\prime}}}$ on $V_{x_{g^{\prime}}}^{*}$ is given as follows: 


$$
\begin{aligned}
& d \rho_{\rho_{9^{\prime}}}(\tilde{A})\left(\omega_{1}, \cdots, \omega_{9}\right)=\left(\omega_{1}, \cdots, \omega_{9}\right)\left(\begin{array}{c|c|c|c}
A-(3 \varepsilon+2 \eta) I_{4} & B & C^{\prime} \\
\hline 0 & -4 \varepsilon & 0 \\
\hline 0 & 0 & A-(\varepsilon+2 \eta) I_{4}
\end{array}\right) \\
& \text { with } C^{\prime}=\left(\begin{array}{c|c|c|c}
c_{13}+2 c_{24} & -c_{23} & 0 & c_{34} \\
\hline-c_{14} & 2 c_{13}+c_{24} & -c_{34} & 0 \\
\hline 0 & -c_{12} & c_{13}+2 c_{24} & -c_{14} \\
\hline c_{12} & 0 & -c_{23} & 2 c_{13}+c_{24}
\end{array}\right) .
\end{aligned}
$$

Clearly, $y_{9}=\omega_{5}+\omega_{6}$ is its generic point and $y_{9}^{\prime}=\omega_{1}+\omega_{8}$ is a point of the one-codimensional orbit. Note that $\left(G_{x_{g^{\prime}}}, \rho_{x_{g^{\prime}}}, V_{x_{9^{\prime}}}^{*}\right)$ has only one orbit of codimension one. Since $y_{9}, y_{9}^{\prime} \in S_{9}^{*}$, we have $\Lambda_{9}=\Lambda_{9}^{*}$, and $\Lambda_{9}$ has no onecodimensional intersection with other conormal bundles. Let $\tilde{A}_{0}$ be an element of $g_{x_{g^{\prime}}}$ with $\varepsilon=-\frac{1}{4}, \eta=-\frac{3}{8}$, all remaining parts zero in (4.7). Then $d \rho\left(\tilde{A}_{0}\right) x_{9}^{\prime}=0$ and $d \rho^{*}\left(\tilde{A}_{0}\right) y_{9}=y_{9}$. We have $\delta \chi\left(\tilde{A}_{0}\right)=2\{(2 \varepsilon+\eta)+\eta\}=$ $-\frac{5}{2}$, we have $m_{A_{9}}=\frac{5}{2}$. This implies that the conormal bundle $\Lambda_{9}$ is not a good holonomic variety, i.e., $\Lambda_{9} \not \subset W$ since otherwise $m_{\Lambda_{9}}$ must be a nonnegative integer (See $\S 1$ or [1]).

(6) The isotropy subalgebra $\mathfrak{g}_{x_{13}}$ at $x_{13}=\left(1, e_{1} e_{2}\right)$ is given as follows.

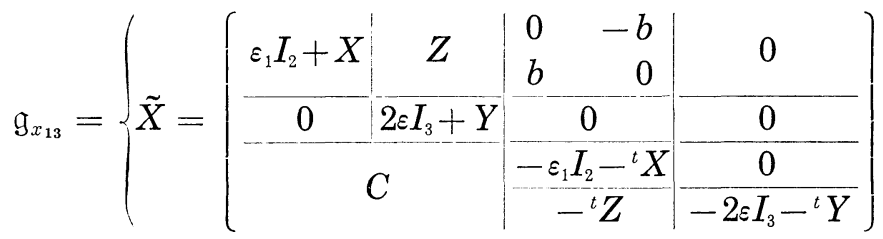

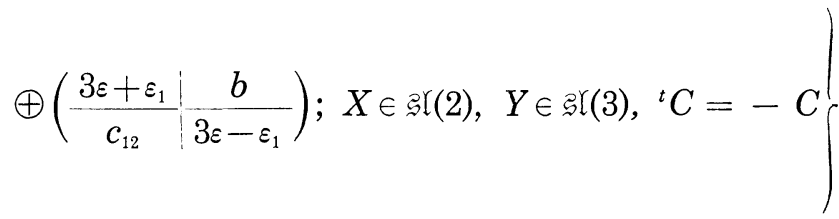

$$
\begin{aligned}
& \cong(\mathfrak{g l}(1) \oplus \mathfrak{g l}(2) \oplus \mathfrak{g} \mathfrak{l}(2) \oplus \mathfrak{g} \mathfrak{l}(3)) \oplus \mathfrak{u}(15) .
\end{aligned}
$$

Since $\Lambda_{13}=\Lambda_{1}^{*}, \Lambda_{8}=\Lambda_{8}^{*}$, and $\operatorname{dim} \Lambda_{1}^{*} \cap \Lambda_{8}^{*}=\operatorname{dim} V-1$, the conormal bundle $\Lambda_{13}$ is a good holonomic variety and $\operatorname{dim} \Lambda_{8} \cap \Lambda_{13}=\operatorname{dim} V-1$. They intersect regularly. Put $\omega_{1}=\left(0, e_{2} e_{3} e_{4} e_{5}\right), \omega_{2}=\left(e_{2} e_{3} e_{4} e_{5}, 0\right), \omega_{3}=\left(0, e_{1} e_{3} e_{4} e_{5}\right)$, $\omega_{4}=\left(e_{1} e_{3} e_{4} e_{5}, 0\right), \omega_{5}=\left(e_{4} e_{5},-e_{1} e_{2} e_{4} e_{5}\right), \omega_{6}=\left(e_{3} e_{5},-e_{1} e_{2} e_{3} e_{5}\right), \omega_{7}=\left(e_{3} e_{4},-e_{1} e_{2} e_{3} e_{4}\right)$, $\omega_{8}=\left(0, e_{4} e_{5}\right), \quad \omega_{9}=\left(0, e_{3} e_{5}\right), \quad \omega_{10}=\left(0, e_{3} e_{4}\right), \omega_{11}=\left(e_{1} e_{2} e_{4} e_{5}, 0\right), \omega_{12}=\left(e_{1} e_{2} e_{3} e_{5}, 0\right)$, $\omega_{13}=\left(e_{1} e_{2} e_{3} e_{4}, 0\right)$. Then the conormal vector space $V_{x_{13}}^{*}$ is spanned by these $\omega_{1}, \cdots, \omega_{13}$ and the action $d \rho_{x_{13}}$ of $\mathfrak{g}_{x_{13}}$ on $V_{x_{13}}^{*}$ is given as follows: 


$$
\begin{aligned}
& d \rho_{x_{13}}(\tilde{X})\left(\omega_{1}, \cdots, \omega_{13}\right) \\
& =\left(\omega_{1}, \cdots, \omega_{13}\right)\left(\begin{array}{c|c}
-6 \varepsilon I_{4}+d \rho_{1}(X \oplus W) & * \\
\hline 0 & -4 \varepsilon I_{9}+d \rho_{2}(W \oplus Y)
\end{array}\right)
\end{aligned}
$$

where $\rho_{1}=\Lambda_{1} \otimes \Lambda_{1}$ for $S L(2) \times S L(2), \rho_{2}=\left(2 \Lambda_{1}\right) \otimes \Lambda_{1}$ for $S L(2) \times S L(3)$ and $W=\left(\begin{array}{cc}\varepsilon_{1} & b \\ c_{12} & -\varepsilon_{1}\end{array}\right) \in \mathfrak{H}(2)$.

As a generic point, we may take $y_{13}=\omega_{5}+\omega_{9}+\omega_{13}=\left(e_{4} e_{5}+e_{1} e_{2} e_{3} e_{4}\right.$, $\left.e_{3} e_{5}-e_{1} e_{2} e_{4} e_{5}\right)$. Let $\tilde{X}_{0}$ be an element of $g_{x_{13}}$ with $\varepsilon=-\frac{1}{4}$, all remaining parts zero. Then $d \rho\left(\tilde{X}_{0}\right) x_{13}=0$ and $d \rho^{*}\left(\tilde{X}_{0}\right) y_{13}=y_{13}$. Since $\delta \chi\left(\tilde{X}_{0}\right)=12 \varepsilon=$ $-3, \operatorname{tr}_{V_{x_{13}}^{*}} \tilde{X}_{0}=-60 \varepsilon=15$ and $\operatorname{dim} V_{x_{13}}^{*}=13$, we have ord $\operatorname{lis}_{13} f^{s}=-3 s-\frac{17}{2}$. By Corollary 1-2, we have $b_{A_{13}}(s) / b_{A_{8}}(s)=(s+5)$. By (4.10), we can see that $\left(G_{x_{13}}, \rho_{x_{13}}, V_{x_{13}}^{*}\right)$ has the unique relative invariant (See Lemma 4 and Proposition 5 in $\S 4$ in [1]), i.e., it has the unique one-codimensional orbit.

(7) The isotropy subalgebra $g_{x_{15}}$ at $x_{15}=\left(1+e_{1} e_{2} e_{3} e_{4}, 0\right)$ is given as follows.

$$
\begin{aligned}
& \mathfrak{g}_{x_{15}}=\left\{\tilde{X}=\left(\begin{array}{c|c|c|c}
X & Y & C^{\prime \prime} & 0 \\
\hline 0 & 2 \varepsilon & 0 & 0 \\
\hline C & C^{\prime} & -{ }^{t} X & 0 \\
\hline-{ }^{t} C^{\prime} & 0 & -{ }^{t} Y & -2 \varepsilon
\end{array}\right) \oplus\left(\begin{array}{cc}
\varepsilon & b \\
0 & \eta
\end{array}\right) ;\right. \\
& \left.X \in \operatorname{gr}(4), C C^{\prime \prime}=-\operatorname{Pf} C \cdot I_{4},{ }^{t} C=-C \in M(4)\right\} \\
& \cong(\mathfrak{g} \mathfrak{l}(1) \oplus \mathfrak{g}(1) \oplus \mathfrak{o}(7)) \oplus \mathfrak{u}(9)
\end{aligned}
$$

Note that, in (4.11), $\tilde{X}_{0}=\left(\begin{array}{c|c}X & C^{\prime \prime} \\ \hline & -{ }^{t} X\end{array}\right)$ is the spin representation of $X_{0}$ in $\mathrm{D}(7)$. Put $\omega_{1}=\left(0, e_{1} e_{5}\right), \omega_{2}=\left(0, e_{2} e_{5}\right), \omega_{3}=\left(0, e_{3} e_{5}\right), \omega_{4}=\left(0, e_{4} e_{5}\right), \omega_{5}=(0$, $\left.e_{2} e_{3} e_{4} e_{5}\right), \quad \omega_{6}=\left(0,-e_{1} e_{3} e_{4} e_{5}\right), \quad \omega_{7}=\left(0, e_{1} e_{2} e_{4} e_{5}\right), \quad \omega_{8}=\left(0,-e_{1} e_{2} e_{3} e_{5}\right), \quad \omega_{9}=(0$, $\left.\frac{1}{2}\left(1-e_{1} e_{2} e_{3} e_{4}\right)\right), \quad \omega_{10}=\left(0, e_{2} e_{3}\right), \quad \omega_{11}=\left(0,-e_{2} e_{4}\right), \quad \omega_{12}=\left(0, e_{3} e_{4}\right), \quad \omega_{13}=\left(0, e_{1} e_{4}\right)$, $\omega_{14}=\left(0, e_{1} e_{3}\right), \omega_{15}=\left(0, e_{1} e_{2}\right)$. The conormal vector space $V_{x_{15}}^{*}$ is spanned by $\omega_{1}, \cdots, \omega_{15}$. Then $y_{15}=\omega_{9}$ is its generic point and $y_{15}^{\prime}=\omega_{10}+\omega_{14}$ is a point of the unique one-codimensional orbit. Since $y_{15}, y_{15}^{\prime} \in S_{15}^{*}$, we have $\Lambda_{15}=$ $\Lambda_{15}^{*}$, and $\Lambda_{15}$ has no one-codimensional intersection with any other conormal bundle. Let $\tilde{X}_{1}$ be an element of $g_{x_{15}}$ with $\varepsilon=\beta+1, \eta=\beta$, all remaining parts zero in (4.11). Then $d \rho\left(\tilde{X}_{1}\right) x_{15}=0$ and $d \rho *\left(\tilde{X}_{1}\right) y_{15}=y_{15}$. Since $\delta \chi\left(\tilde{X}_{1}\right)$ $=2(\varepsilon+\eta)=2(2 \beta+1)$ is not definite, the conormal bundle $\Lambda_{15}$ is not a good holonomic variety, i.e., $\Lambda_{15} \not \subset W$. 
(8) Since $\Lambda_{20}=\Lambda_{4}^{*}$ and $\Lambda_{4} \not \subset W$, the conormal bundle $\Lambda_{20}$ is not a good holonomic variety. Note that $W \subset V \times V^{*}$ is symmetric with respect to $V$ and $V^{*}$.

(9) Since $\Lambda_{32}=\{0\} \times V^{*}$, the conormal bundle $\Lambda_{32}$ is a good holonomic variety. Put $A_{0}=(0) \oplus\left(-I_{2}\right)$. Then $d \rho\left(A_{0}\right) x_{32}=0$ and $d \rho^{*}\left(A_{0}\right) y_{32}=y_{32}$ where $y_{32}$ is a generic point of $\left(G, \rho^{*}, V^{*}\right)$. Since $\delta \chi\left(A_{0}\right)=-4, \operatorname{tr}_{V_{x_{32}}^{*}} A_{0}=32$ and $\operatorname{dim} V_{x_{32}}^{*}=32$, we have $\operatorname{ord}_{\Lambda_{32}} f^{s}=-4 s-\frac{32}{2}$ and hence by Corollary 1-2, we have $b_{\Lambda_{32}}(s) / b_{\Lambda_{13}}(s)=s+8$. Note that $\Lambda_{32}=\Lambda_{0}^{*}$ and $\Lambda_{13}=\Lambda_{1}^{*}$. Since $b_{\Lambda_{0}}(s)=1$ and $b_{\Lambda_{32}}(s)=b(s)$, we have the $b$-function $b(s)=(s+1)(s+4)$ $(s+5)(s+8)$, and the holonomy diagram (Figure $4-1)$. We denote $\Lambda_{m}$ by (m).

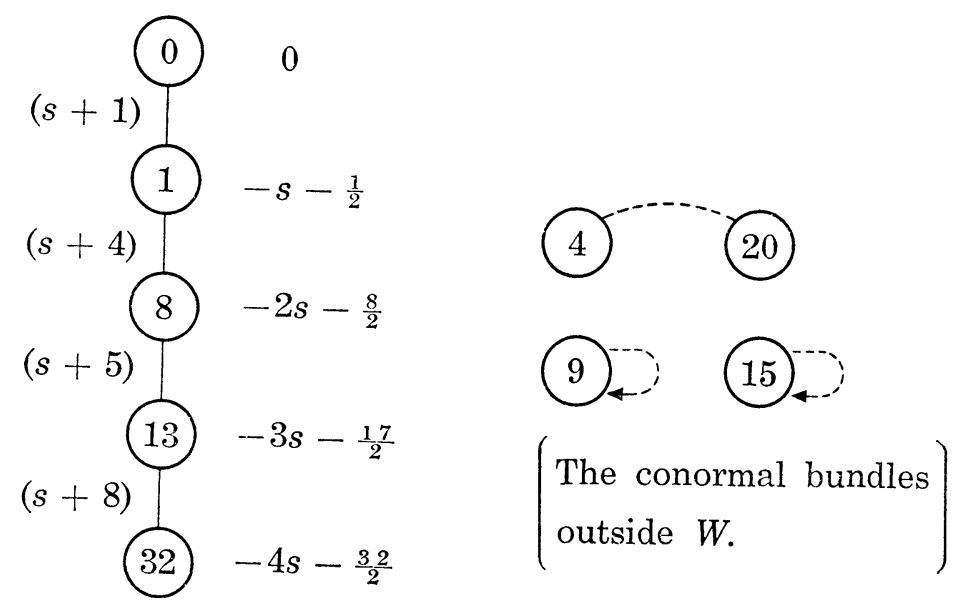

Figure 4-1. Holonomy diagram of $\left(\operatorname{Spin}(10) \times G L(2)\right.$, half-spin rep. $\left.\otimes \Lambda_{1}, V(16) \otimes V(2)\right)$

\section{§5. $(G L(1) \times$ Spin(12), $\square \otimes$ half-spin rep., $V(1) \otimes V(32))$}

The representation space $V=V(1) \otimes V(32)$ is spanned by $1, e_{i} e_{j}, e_{r} e_{s} e_{t} e_{u}$, $e_{1} e_{2} e_{3} e_{4} e_{5} e_{6}(1 \leq i<j \leq 6,1 \leq r<s<t<u \leq 6)$ (See [1], [4]). J-I. Igusa has completed the orbital decomposition of this space (See [3]). There exist five $G$-orbits $S_{m}=\rho(G) x_{m}(m=0,1,7,16,32)$ where $S_{m}$ denotes the $m$-codimensional orbit and $x_{n}=1+e_{1} e_{2} e_{3} e_{4} e_{5} e_{6}, x_{1}=1+e_{2} e_{3} e_{5} e_{6}+e_{1} e_{3} e_{4} e_{6}, x_{7}$ $=1+e_{2} e_{3} e_{5} e_{6}, x_{16}=1, x_{32}=0$. We identify $V^{*}$ with $V$ by taking $\left\{1, e_{i} e_{j}\right.$, $\left.e_{r} e_{s} e_{t} e_{u}, e_{1} e_{2} e_{3} e_{4} e_{5} e_{6}\right\}$ as a dual basis. Since $(G, \rho, V) \cong\left(G, \rho^{*}, V^{*}\right)$, there exist also five orbits $S_{m}^{*}(m=0,1,7,16,32)$ in $V^{*}$. We denote by $\Lambda_{m}$ (resp. $\left.\Lambda_{m}^{*}\right)$ the conormal bundle of $S_{m}$ (resp. $S_{m}^{*}$ ). Clearly, we have $\Lambda_{0}=V \times\{0\}=$ $\Lambda_{32}^{*}$ and $\Lambda_{32}=\{0\} \times V^{*}=\Lambda_{0}^{*}$. The Lie algebra $g$ of $G L(1) \times \operatorname{Spin}(12)$ is given as follows: 


$$
\mathfrak{g}=\left\{(d) \oplus\left(\frac{A}{C} \mid \frac{B}{-{ }^{t} A}\right) ; A, B, C \in M(6),{ }^{t} B=-B,{ }^{t} C=-C\right\}
$$

(1) The isotropy subalgebra $g_{x_{0}}$ at $x_{0}$ is given as follows (See [1]).

$$
\mathfrak{g}_{x_{0}}=\left\{(0) \oplus\left(\frac{A}{0} \mid \frac{0}{-{ }^{t} A}\right) ; A \in \mathfrak{I l}(6)\right\} \cong \mathfrak{g l}(6) .
$$

Since $\Lambda_{0}=V \times\{0\}$, we have $\operatorname{ord}_{\Lambda_{0}} f^{s}=0$, where $f$ denotes the relative invariant of degree four (See [1], [3]).

(2) By using (5.29) in [1], we can calculate the isotropy subalgebra $\mathfrak{g}_{x_{1}}$.

$$
\begin{aligned}
& \mathfrak{g}_{x_{1}}=\left\{\tilde{A}=(d) \oplus\left\{\frac{A}{C} \mid \frac{B}{-{ }^{t} A}\right\} ; a_{1}+a_{4}=a_{2}+a_{5}=-a_{3}-a_{6}=2 d, c_{36}=0\right\} \\
&(5.3) \quad \cong\left\{(d) \oplus\left(\frac{d I_{6}+A_{\bullet}}{0} \mid \frac{B_{0}}{-d I_{6}-{ }^{t} A_{0}}\right) ; A_{0} \in \mathfrak{S p}(3),{ }^{t} B_{0}=-B_{n}, \operatorname{tr} B_{0} J=0\right\} \\
& \quad \text { with } \quad J=\left(\frac{0}{-I_{3}} \mid \frac{I_{3}}{0}\right), \\
& \cong(\mathfrak{g r}(1) \oplus \mathfrak{g p}(3)) \oplus V(14) \quad \text { where }
\end{aligned}
$$

$$
A=\left(\begin{array}{llllll}
a_{1} & a_{12} & 0 & a_{14} & a_{15} & 0 \\
a_{21} & a_{2} & 0 & a_{15} & a_{25} & 0 \\
a_{31} & a_{32} & a_{3} & a_{34} & a_{35} & a_{36} \\
a_{41} & a_{42} & 0 & a_{4} & -a_{21} & 0 \\
a_{42} & a_{52} & 0 & -a_{12} & a_{5} & 0 \\
a_{61} & a_{62} & a_{63} & a_{64} & a_{65} & a_{6}
\end{array}\right), B=\left(\begin{array}{cccccc}
0 & 0 & c_{46} & 0 & 0 & c_{34} \\
0 & 0 & c_{56} & 0 & 0 & c_{35} \\
-c_{46} & -c_{56} & 0 & c_{16} & c_{26} & b_{36} \\
0 & 0 & -c_{16} & 0 & 0 & c_{13} \\
0 & 0 & -c_{26} & 0 & 0 & c_{23} \\
-c_{34} & -c_{35} & -b_{36} & -c_{13} & -c_{23} & 0
\end{array}\right)
$$

with $b_{36}+c_{14}+c_{25}=0$.

The conormal vector space $V_{x_{1}}^{*}$ is spanned by $e_{1} e_{2} e_{4} e_{5}$ on which $\mathfrak{g}_{x_{1}}$ acts as $d \rho_{x_{1}}(\tilde{A}) e_{1} e_{2} e_{4} e_{5}=-4 d e_{1} e_{2} e_{4} e_{5}$ for $\tilde{A} \in \mathfrak{g}_{x_{1}}$. This implies that $\Lambda_{1}=\overline{G\left(x_{1}, e_{1} e_{2} e_{4} e_{5}\right)}$ $=\Lambda_{16}^{*}$. Since 0 is the point of the one-codimensional orbit, we have $\operatorname{dim} \Lambda_{0} \cap \Lambda_{1}=\operatorname{dim} V-1$ and $\Lambda_{0} \cap \Lambda_{1}$ is $G_{0}$-prehomogeneous, i.e., $\Lambda_{1}$ is a good holonomic variety by Proposition 1-5. Let $A_{0}$ be an element of $\mathfrak{g}_{x_{1}}$ with $-4 d=1$. Then $d \rho\left(A_{0}\right) x_{1}=0$ and $d \rho^{*}\left(A_{0}\right) y_{1}=y_{1}$ where $y_{1}=e_{1} e_{2} e_{4} e_{5}$. Since $\delta \chi\left(A_{0}\right)=4 d=-1, \operatorname{tr}_{V_{x_{1}}^{*}} A_{0}=\operatorname{dim} V_{x_{1}}^{*}=1$, we have $\operatorname{ord}_{\Lambda_{1}} f^{s}=-s-\frac{1}{2} . \quad$ By Proposition 1-4, $\Lambda_{0}$ and $\Lambda_{1}$ intersect regularly and hence $b_{\Lambda_{1}}(s) / b_{\Lambda_{0}}(s)=$ $(s+1)$ by Corollary 1-2.

(3) By using (5.29) in [1], we can calculate the isotropy subalgebra $\mathfrak{g}_{x_{7}}$.

$$
\mathrm{g}_{x_{7}}=\left\{\tilde{A}=(d) \oplus\left(\begin{array}{c|c}
A & C^{\prime} \\
\hline C & -{ }^{t} A
\end{array}\right) ; d=\frac{a_{1}+a_{4}}{2}, a_{2}+a_{3}+a_{5}+a_{6}=0,\right.
$$




$$
\begin{aligned}
& \left.{ }^{t} C=C, A \text { and } C^{\prime} \text { are given as follows }\right\} \\
\cong & \left\{(d) \oplus\left(\begin{array}{c|c|c}
d I_{2}+A_{1} & 0 & 0 \\
\hline U & d \rho_{1}(V) & 0 \\
\hline W & -{ }^{t} U S & -d I_{2}-{ }^{t} A_{1}
\end{array}\right) ; \begin{array}{l}
A_{1} \in \mathfrak{g}((2), W \in \mathfrak{v}(2), \\
V \in \mathfrak{o}(7), U \in M(8.2)
\end{array}\right\} \\
\cong & (\mathfrak{g l}(1) \oplus \mathfrak{o}(7) \oplus \mathfrak{g l}(2)) \oplus \mathfrak{u}(17)
\end{aligned}
$$

where $\rho_{1}$ is the spin-representation of $\operatorname{Spin}(7), S=\left(\begin{array}{ll}0 & I_{4} \\ I_{4} & 0\end{array}\right)$, and

$$
A=\left(\begin{array}{llllll}
a_{1} & 0 & 0 & a_{14} & 0 & 0 \\
a_{21} & a_{2} & a_{23} & a_{24} & a_{25} & a_{26} \\
a_{31} & a_{32} & a_{3} & a_{34} & a_{35} & a_{36} \\
a_{41} & 0 & 0 & a_{4} & 0 & 0 \\
a_{51} & a_{52} & a_{53} & a_{54} & a_{5} & a_{56} \\
a_{61} & a_{62} & a_{63} & a_{64} & a_{65} & a_{6}
\end{array}\right), \quad C^{\prime}=\left(\begin{array}{cccccc}
0 & 0 & 0 & 0 & 0 & 0 \\
0 & 0 & c_{56} & 0 & -c_{36} & c_{35} \\
0 & -c_{56} & 0 & 0 & c_{26} & -c_{25} \\
0 & 0 & 0 & 0 & 0 & 0 \\
0 & c_{36} & -c_{26} & 0 & 0 & c_{23} \\
0 & -c_{35} & c_{25} & 0 & -c_{23} & 0
\end{array}\right)
$$

Put $\omega_{1}=e_{1} e_{4}-e_{1} e_{2} e_{3} e_{4} e_{5} e_{6}, \omega_{2}=e_{1} e_{2} e_{4} e_{5}, \omega_{3}=e_{1} e_{2} e_{3} e_{4}, \omega_{4}=e_{1} e_{2} e_{4} e_{6}, \omega_{5}=e_{1} e_{3} e_{4} e_{5}$, $\omega_{6}=e_{1} e_{3} e_{4} e_{6}, \omega_{7}=e_{1} e_{4} e_{5} e_{6}$. The conormal vector space $V_{x_{7}}^{*}$ is spanned by these $\omega_{1}, \cdots, \omega_{7}$, and $\left(G_{x_{7}}, \rho_{x_{7}}, V_{x_{7}}^{*}\right) \cong\left(G L(1) \times S O(7), \square \otimes \Lambda_{1}, V(1) \otimes V(7)\right)$. Then $\omega_{1}$ is its generic point and $\omega_{2}=e_{1} e_{2} e_{4} e_{5}$ is a point of the one-codimensional orbit. Since $\Lambda_{1}=\overline{G\left(x_{1}, \omega_{2}\right)}$, we have codim $\Lambda_{1} \cap \Lambda_{7}=1$. Since $\Lambda_{1} \cap \Lambda_{7}$ is $G_{0}$-prehomogeneous, $\Lambda_{7}$ is a good holonomic variety. We have $\Lambda_{7}=\Lambda_{7}^{*}$.

Let $A_{\beta}^{\prime}$ be an element of $\mathfrak{g}_{x_{7}}$ with $4 d=2\left(a_{1}+a_{4}\right)=-\beta-1,2\left(a_{3}+a_{6}\right)$ $=-2\left(a_{2}+a_{5}\right)=1-\beta$, all remaining parts zero in (5.4). Then we have $d \rho\left(A_{\beta}^{\prime}\right) x_{7}=0, d \rho^{*}\left(A_{\beta}^{\prime}\right) \omega_{2}=\omega_{2}$ and $\operatorname{tr}_{\tilde{v}} A_{\beta}^{\prime}=\beta$ where $\tilde{V}=V_{x_{7}}^{*} \bmod d \rho_{x_{7}}\left(\mathfrak{g}_{x_{7}}\right) \omega_{2}$. This implies that $\Lambda_{1}$ and $\Lambda_{7}$ intersect regularly by Proposition 1-4. Let $A_{0}$ be an element of $g_{x_{7}}$ with $d=-\frac{1}{2}$, all remaining parts zero in (5.4). Then $d \rho\left(A_{0}\right) x_{7}=0, d \rho^{*}\left(A_{0}\right) \omega_{1}=\omega_{1}$ Since $\delta \chi\left(A_{0}\right)=4 d=-2, \operatorname{tr}_{V_{x_{7}}^{*}} A_{0}=-14 \mathrm{~d}=$ $7, \operatorname{dim} V_{x_{7}}^{*}=7$, we have $\operatorname{ord}_{A_{7}} f^{s}=-2 s-\frac{7}{2}$. By Corollary 1-2, we have $b_{\Lambda_{7}}(s) / b_{\Lambda_{1}}(s)=\left(s+\frac{7}{2}\right)$.

(4) $\operatorname{Since}(G, \rho, V) \cong\left(G, \rho^{*}, V^{*}\right), \Lambda_{7}^{*}=\Lambda_{7}$ and $\Lambda_{1}^{*}=\Lambda_{16}$, we have $\operatorname{dim} \Lambda_{7}$ $\cap \Lambda_{16}=\operatorname{dim} V-1$ and they intersect regularly. Since $\Lambda_{7} \cap \Lambda_{16}=\Lambda_{1}^{*} \cap \Lambda_{7}^{*}$ is $G_{0}$-prehomogeneous, $\Lambda_{16}$ is a good holonomic variety. Since $d \rho(\tilde{A}) \cdot 1=d$ - $(\operatorname{tr} A / 2)+\sum_{i<j} b_{i j} e_{i} e_{j}$, the isotropy subalgebra $g_{x_{16}}$ at $x_{16}=1$ is

$$
\begin{aligned}
\mathfrak{g}_{x_{16}}= & \left\{\tilde{A}=\left(\frac{\operatorname{tr} A}{2}\right) \oplus\left(\begin{array}{cc}
A & 0 \\
C & -{ }^{t} A
\end{array}\right) ;{ }^{t} C=-C, A, C \in M(3)\right\} \\
& \cong \mathfrak{g l}(6) \oplus V(15) .
\end{aligned}
$$


The conormal vector space $V_{x_{18}}^{*}$ is spanned by $e_{1} e_{2} e_{3} e_{4} e_{5} e_{6}$ and $e_{i} e_{j} e_{k} e_{\ell}(1 \leq$ $i<j<k<\ell \leq 6)$. Then the action $d \rho_{x_{16}}$ of $g_{x_{16}}$ on $V_{x_{18}}^{*}$ is given by

$$
d \rho_{x_{16}}(\tilde{A})\left(\omega_{1}, \cdots, \omega_{16}\right)=\left(\omega_{1}, \cdots, \omega_{16}\right)\left(\begin{array}{cc}
-\operatorname{tr} A & c^{\prime} \\
0 & d \rho_{1}^{*}(A)
\end{array}\right)
$$

where $\omega_{1}=e_{1} e_{2} e_{3} e_{4} e_{5} e_{6},\left\{\omega_{2}, \cdots, \omega_{16}\right\}=\left\{e_{i} e_{j} e_{k} e_{\ell}, 1 \leq i<j<k<\ell \leq 6\right\},{ }^{t} c^{\prime} \in C^{15}$, $\rho_{1}=\Lambda_{2}$ for $G L(6)$.

Then $y_{16}=e_{1} e_{2} e_{4} e_{5}+e_{1} e_{3} e_{4} e_{6}+e_{2} e_{3} e_{5} e_{6}$ is its generic point. Let $A_{0}$ be an element of $\mathfrak{g}_{x_{16}}$ with $A=-\frac{1}{4} I_{6}, C=0$ in (5.5). Then $d_{\rho}\left(A_{0}\right) x_{16}=0$ and $d \rho^{*}\left(A_{0}\right) y_{16}=y_{16}$. Since $\delta \chi\left(A_{0}\right)=4 d=2 \operatorname{tr}\left(-\frac{1}{4} I_{6}\right)=-3, \operatorname{tr}_{V x_{16}}^{*} A_{0}=$ $-11 \operatorname{tr}\left(-\frac{1}{4} I_{6}\right)=\frac{33}{2}$ and $\operatorname{dim} V_{x_{16}^{*}}=16$, we have $\operatorname{ord}_{A_{18}} f^{s}=\operatorname{so} \chi\left(A_{0}\right)-\operatorname{tr}_{V_{x_{16}}^{*}} A_{0}+$ $\frac{1}{2} \operatorname{dim} V_{x_{16}^{*}}=-3 s-\frac{17}{2}$. By Corollary 1-2, we have $b_{A_{16}}(s) / b_{A_{7}}(s)=\left(s+\frac{11}{2}\right)$. By (5.6), the character group of $\rho_{x_{16}}\left(G_{x_{16}}\right)$ is one-dimensional and hence $\left(G_{x_{16}}, \rho_{x_{16}}, V_{x_{16}}^{*}\right)$ has (at most) the unique one-codimensional orbit.

(5) Since $\Lambda_{32}=\Lambda_{0}^{*}$ and $\Lambda_{16}=\Lambda_{1}^{*}$, they intersect regularly with codimension one. We shall calculate the order $\operatorname{ord}_{4_{32}} f^{s}$. Since $\left(G_{x_{32}}, \rho_{x_{32}}, V_{x_{32}}\right)$ $\cong\left(G, \rho^{*}, V^{*}\right), \quad y_{32}=1+e_{1} e_{2} e_{3} e_{4} e_{5} e_{6}$ is its generic point. Let $A_{0}$ be an element of $g$ with $d=-1$, all remaining parts zero in (5.1). Then $d \rho\left(A_{0}\right) x_{32}$ $=0, d \rho^{*}\left(A_{0}\right) y_{32}=y_{32}$. Since $\delta \chi\left(A_{0}\right)=-4, \operatorname{tr}_{V_{32}^{*}} A_{0}=-32 d=32, \operatorname{dim} V_{x_{32}^{*}}=$ 32 , we have $\operatorname{ord}_{\Lambda_{32}} f^{s}=-4 s-\frac{32}{2}$. By Corollary 1-2, we have $b_{\Lambda_{32}}(s) / b_{A_{16}}(s)$ $=s+8$. Since $b_{A_{0}}(s)=1$ and $b_{A_{32}}(s)=b(s)$, we obtain the $b$-function $b(s)$ $=(s+1)\left(s+\frac{7}{2}\right)\left(s+\frac{11}{2}\right)(s+8)$ and the holonomy diagram (Figure 5-1). We denote $\Lambda_{m}$ by $m$.

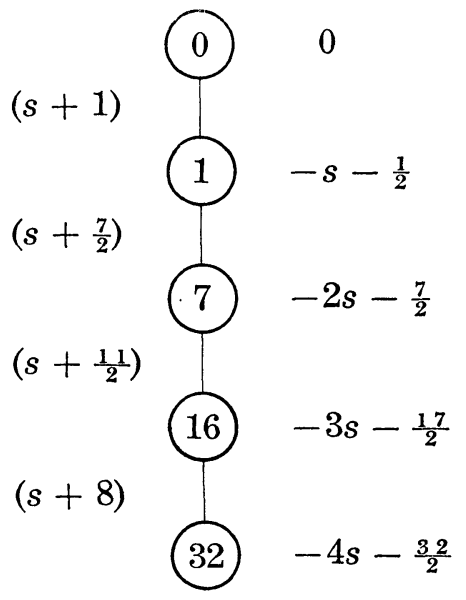

Figure 5-1. Holonomy diagram of $(G L(1) \times \operatorname{Spin}(12)$, $\square \otimes$ half-spin rep., $V(1) \otimes V(32))$. 
Remark. (1) $(G L(1) \times \operatorname{Spin}(7), \square \otimes \operatorname{spin}$ rep., $V(1) \otimes V(8))$

(2) $\left(\operatorname{Spin}(7) \times G L(2)\right.$, spin rep. $\left.\otimes \Lambda_{1}, V(8) \otimes V(2)\right)$

(3) $\left(\operatorname{Spin}(7) \times G L(3)\right.$, spin rep. $\left.\otimes \Lambda_{1}, V(8) \otimes V(3)\right)$

(4) $(G L(1) \times \operatorname{Spin}(9), \square \otimes \operatorname{spin}$ rep., $V(1) \otimes V(16))$

(5) $\left(G L(1) \times\left(G_{2}\right), \square \otimes \Lambda_{2}, V(1) \otimes V(7)\right)$

(6) $\left(\left(G_{2}\right) \times G L(2), \Lambda_{2} \otimes \Lambda_{1}, V(7) \otimes V(2)\right)$

(7) $(G L(1) \times \operatorname{Spin}(11), \square \otimes \operatorname{spin}$ rep., $V(1) \otimes V(32))$

Since $\operatorname{Spin}(7) \longrightarrow S O(8)$ by the spin representation, the first three triplets (1), (2), (3) are reduced to the triplet $\left(S O(8) \times G L(n), \Lambda_{1} \otimes \Lambda_{1}, V(8) \otimes V(n)\right)$ $(n=1,2,3)$ (See [1]). Since $\operatorname{Spin}(9) \smile S O(16)$ by the spin representation, (4) is reduced to $\left(S O(16) \times G L(1), \Lambda_{1} \otimes \Lambda_{1}, V(16) \otimes V(1)\right)$ (See [1]). Since $\left(G_{2}\right) \smile S O(7)$ by $\Lambda_{2},(5)$ and (6) are reduced to $\left(S O(7) \times G L(n), \Lambda_{1} \otimes \Lambda_{1}\right.$, $V(7) \otimes V(n))(n=1,2)$ (See [1]).

Since the spin representation of $\operatorname{Spin}(11)$ is obtained by the restriction of the half-spin representation of $\operatorname{Spin}(12)$ to $\operatorname{Spin}(11)$, (7) is reduced to Spin (12) in $\S 5$. Note that the $b$-function depends essentially on the relative invariant itself, not on the group.

\section{§ 6. $\left(G L(1) \times E_{6}, \square \otimes \Lambda_{1}, V(1) \otimes V(27)\right)$}

The Lie algebra $\mathfrak{g}$ of $G=G L(1) \times E_{6}$ can be written as $\mathfrak{g}=\mathscr{D}_{0} \oplus \mathscr{T}_{1}$ $\oplus \mathscr{T}_{2} \oplus \mathscr{T}_{3} \oplus\left\{R_{y}\right\}_{y \in \mathscr{S}}$ (See Proposition 37 and Example 39 of $\S 1$ in [1]). The representation space is identified with the simple Jordan algebra $\mathscr{J}$.

$$
\mathscr{J}=\left\{X=\left(\begin{array}{ccc}
\xi_{1} & x_{3} & \bar{x}_{2} \\
\bar{x}_{3} & \xi_{2} & x_{1} \\
x_{2} & \bar{x}_{1} & \xi_{3}
\end{array}\right) ; \xi_{1}, \xi_{2}, \xi_{3} \in C ; x_{1}, x_{2}, x_{3} \in \mathscr{L}\right\}
$$

where $\mathscr{L}$ denotes the complex Cayley algebra.

Definition 6-1. For $a \in \mathscr{L}$, we define elements $T_{i}(a)$ and $T_{i}^{\prime}(a)(i=1$, $2,3)$ of $\mathfrak{g}$ as follows:

$$
\begin{aligned}
& T_{1}(a) \cdot X=\left[R_{A_{1}(a)}+\mathscr{T}_{1}(2 a)\right] X=\left(\begin{array}{ccc}
0 & 0 & x_{3} a \\
0 & 0 & a \xi_{2} \\
\overline{x_{3} a} & \overline{a \xi_{2}} & \operatorname{tr}\left(\bar{x}_{1} a\right)
\end{array}\right) \\
& T_{1}^{\prime}(a) \cdot X=\left[R_{A_{1}(a)}-\mathscr{T}_{1}(2 a)\right] X=\left(\begin{array}{ccc}
0 & \overline{a x_{2}} & 0 \\
a x_{2} & \operatorname{tr}\left(a \bar{x}_{1}\right) & a \xi_{3} \\
0 & \overline{a \xi_{3}} & 0
\end{array}\right) \\
& T_{2}(a) \cdot X=\left[R_{A_{2}(\bar{a})}+\mathscr{T}_{2}(2 \bar{a})\right] X=\left(\begin{array}{ccc}
0 & 0 & a \xi_{1} \\
0 & 0 & \bar{x}_{3} a \\
\overline{a \xi_{1}} & \bar{a} x_{3} & \operatorname{tr}\left(x_{2} a\right)
\end{array}\right)
\end{aligned}
$$




$$
\begin{aligned}
& T_{2}^{\prime}(a) \cdot X=\left[R_{A_{2}(\bar{a})}-\mathscr{T}_{2}(2 \bar{a})\right] X=\left(\begin{array}{ccc}
\operatorname{tr}\left(a x_{2}\right) & a \bar{x}_{1} & a \xi_{3} \\
x_{1} \bar{a} & 0 & 0 \\
\frac{a \xi_{3}}{3} & 0 & 0
\end{array}\right) \\
& T_{3}(a) \cdot X=\left[R_{A_{3}(a)}+\mathscr{T}_{3}(2 a)\right] X=\left(\begin{array}{ccc}
\frac{0}{a \xi_{1}} & \operatorname{tr}\left(\bar{a} x_{3}\right) & \frac{0}{x_{2} a} \\
0 & x_{2} a & 0
\end{array}\right) \\
& T_{3}^{\prime}(a) \cdot X=\left[R_{A_{3}(a)}-\mathscr{T}_{3}(2 a)\right] X=\left(\begin{array}{ccc}
\operatorname{tr}\left(a \bar{x}_{3}\right) & a \xi_{2} & a x_{1} \\
\overline{a \xi_{2}} & 0 & 0 \\
\frac{\overline{a x}}{x_{1}} & 0 & 0
\end{array}\right)
\end{aligned}
$$

where $A_{i}(a)$ denotes the element of $\mathscr{J}$ with $x_{i}=a$, all remaining terms zero in (6.1) for $i=1,2,3$, and $\operatorname{tr} b=b+\bar{b}$ for $b \in \mathscr{L}$. Thus we have $g$ $\left.=\mathscr{D}_{0} \oplus T_{1} \oplus T_{2} \oplus T_{3} \oplus T_{1}^{\prime} \oplus T_{2}^{\prime} \oplus T_{3}^{\prime} \oplus\left\{\begin{array}{ccc}n_{1} & 0 & 0 \\ 0 & \eta_{2} & 0 \\ 0 & 0 & \eta_{3}\end{array}\right)\right\}$. For $a \in \mathscr{L}$, we put $t_{i}(a)=$ $\exp T_{i}(a)$ and $t_{i}^{\prime}(a)=\exp T_{i}^{\prime}(a)$ for $i=1,2,3$. They are elements of $G$. For $\xi \in C$, let $B_{i}(\xi)$ be the element of $\mathscr{J}$ with $\xi_{i}=\xi$, all remaining terms zero in (6.1) for $i=1,2,3$ and put $c=\exp \xi$. We define an element $S_{i}(c)$ of $G$ by $S_{i}(c)=\exp R_{B_{i}(\xi)}$ for $i=1,2,3$. The following proposition is wellknown.

Proposition 6-2. There exist four orbits $S_{m}=\rho(G) x_{m}(m=0,1,10,27)$ where $S_{m}$ denotes the m-codimensional orbit, and $x_{m}$ is given as follows:

$$
x_{0}=\left(\begin{array}{lll}
1 & & \\
& 1 & \\
& & 1
\end{array}\right), x_{1}=\left(\begin{array}{lll}
1 & & \\
& 1 & \\
& & 0
\end{array}\right), x_{10}=\left(\begin{array}{lll}
1 & & \\
& 0 & \\
& & 0
\end{array}\right), x_{27}=(0) .
$$

Proof. Let $X$ be a non-zero element of $\mathscr{J}$. Then we may assume that $\xi_{1}=1$ by $t_{i}, t_{i}^{\prime}$ and $S_{1}$. By $t_{2}\left(-\bar{x}_{2}\right)$ and $t_{3}\left(-x_{3}\right)$, we have $x_{2}=x_{3}=0$. Unless $\xi_{2}=\xi_{3}=x_{1}=0$, we have $\xi_{2}=1$ by $t_{1}^{\prime}$ and $S_{2}$. Then by $t_{1}\left(-x_{1}\right)$, we have $x_{1}=0$. If $\xi_{3} \neq 0$, we have $\xi_{3}=1$ by $S_{3}$. Thus we obtain four orbits. We shall calculate their codimension later. Q.E.D.

Definition 6-3. We identify the dual vector space $V^{*}$ of $V=\mathscr{J}$ with $V$ by $\langle X, Y\rangle=\operatorname{tr} X \circ Y$. Then the dual actions are given as follows: (i) $D^{*} Y=D Y$ for $D \in \mathscr{D}_{0}$. (ii) $T_{i}^{*}(a) Y=-T_{i}^{\prime}(a) Y$ for $a \in \mathscr{L}$ and $i=1,2,3$. (iii) $T_{i}^{\prime *}(a) Y=-T_{i}(a) Y$ for $i=1,2,3$ and $a \in \mathscr{L}$. (iv) $R_{z}^{*} Y=-R_{z} Y$ for $z \in \mathscr{J}$.

Definition 6-4. Since $(G, \rho, V) \cong\left(G, \rho^{*}, V^{*}\right)$, the dual space has also four orbits $S_{m}^{*}(m=0,1,10,27)$. We denote by $\Lambda_{m}$ (resp. $\left.\Lambda_{m}^{*}\right)$ the conormal 
bundle of $S_{m}$ (resp. $S_{m}^{*}$. Clearly we have $\Lambda_{0}=V \times\{0\}=\Lambda_{27}^{*}$ and $\Lambda_{27}=\{0\}$ $\times V^{*}=\Lambda_{0}^{*}$.

(1) The isotropy subalgebra $\mathfrak{g}_{x_{0}}$ at $x_{0}$ is $\mathscr{D}_{0}$ which is the Lie algebra of $F_{4}$ (See [1]). Since $\Lambda_{0}=V \times\{0\}$, we have $\operatorname{ord}_{\Lambda_{0}} f^{s}=0$.

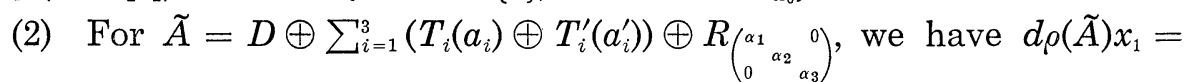
$\left(\begin{array}{ccc}\frac{\alpha_{1}}{a_{3}+a_{3}^{\prime}} & a_{3}+a_{3}^{\prime} & a_{2} \\ \bar{a}_{2} & \alpha_{2} & a_{1} \\ \bar{a}_{1} & 0\end{array}\right)$, and hence $\tilde{A}$ is an element of the isotropy subalgebra $\mathfrak{g}_{x_{1}}$ at $x_{1}$ if and only if $\tilde{A}=D \oplus T_{1}^{\prime}\left(a_{1}^{\prime}\right) \oplus T_{2}^{\prime}\left(a_{2}^{\prime}\right) \oplus\left[T_{3}\left(a_{3}\right) \oplus T_{3}^{\prime}\left(-a_{3}\right)\right] \oplus R\left({ }_{{ }_{0}{ }_{\alpha_{3}}}\right)$. The conormal vector space $V_{x_{1}}^{*}$ is given by $V_{x_{1}}^{*}=\left\{\left(\begin{array}{lll}0 & & 0 \\ & 0 & \\ 0 & & \eta\end{array}\right) ; \eta \in C\right\} \cong\{\eta\}$ and $d \rho_{x_{10}}(\tilde{A}) \eta=-\alpha_{3} \eta$. For $\tilde{A}_{0}=R\left({ }_{0_{-1}}\right)$, we have $d \rho\left(\tilde{A}_{0}\right) x_{1}=0$ and $d \rho^{*}\left(\tilde{A}_{0}\right) y_{1}$ $=y_{1}$ where $y_{1}=\left(\begin{array}{lll}0 & & \\ & 0 & \\ & & 1\end{array}\right)$. Since $\delta \chi\left(\tilde{A}_{0}\right)=-1, \operatorname{tr}_{V_{x_{1}}^{*}} \tilde{A}_{0}=\operatorname{dim} V_{x_{1}}^{*}=1$, we have $\operatorname{ord}_{\Lambda_{1}} f^{s}=-s-\frac{1}{2}$. By Corollary 1-2, we have $b_{\Lambda_{1}}(s) / b_{\Lambda_{0}}(s)=(s+1)$.

(3) For $\tilde{A}=D \oplus \sum_{i=1}^{3}\left(T_{i}\left(a_{i}\right) \oplus T_{i}^{\prime}\left(a_{i}^{\prime}\right)\right) \oplus R_{\left({ }_{\alpha_{2}}^{\alpha_{\alpha_{3}}}\right)}$, we have $d \rho(\tilde{A}) x_{10}=$ $\left(\begin{array}{lll}\alpha_{1} & a_{3} & a_{2} \\ \bar{a}_{3} & 0 & 0 \\ \bar{a}_{2} & 0 & 0\end{array}\right)$ and hence $\tilde{A} \in \mathfrak{g}_{x_{10}}$ if and only if $\tilde{A}=D \oplus T_{1}\left(a_{1}\right) \oplus T_{1}^{\prime}\left(a_{1}^{\prime}\right) \oplus T_{2}^{\prime}\left(a_{2}^{\prime}\right)$ $\left.\oplus T_{3}^{\prime}\left(a_{3}^{\prime}\right) \oplus R\left(R_{\alpha_{2}}^{0}\right)_{\alpha_{3}}\right)$. In this case, $\tilde{A}$ acts on the conormal vector space as follows:

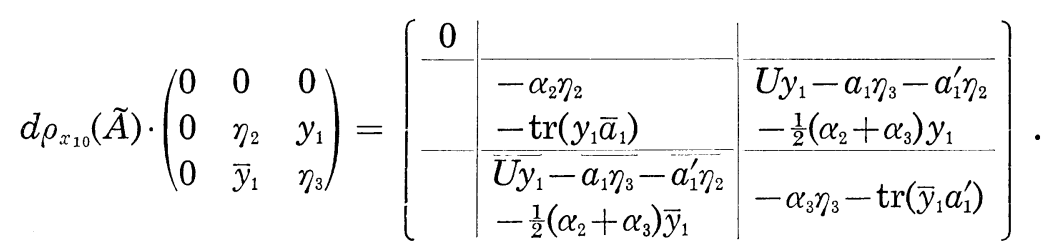

Let $y_{1 \mathrm{n}}$ (resp. $y_{10}^{\prime}$ ) be the element of $\mathscr{J}$ with $x_{1}=1$ (resp. $\xi_{3}=1$ ), all remaining parts zero in (6.1). Then $y_{10}$ is a generic point of $\left(G_{x_{10}}, \rho_{x_{10}}, V_{x_{10}}^{*}\right)$ and $y_{10}^{\prime}$ is a point of the one-codimensional orbit. Thus we have $\Lambda_{10}=\Lambda_{1}^{*}$, $\Lambda_{1}=\Lambda_{10}^{*}$ and $\operatorname{dim} \Lambda_{1} \cap \Lambda_{10}=\operatorname{dim} V-1$. Put $\tilde{A}_{0}=R\left({ }_{-1}{ }_{-1}\right)$. Then we have $d \rho\left(\tilde{A}_{0}\right) x_{10}=0$ and $d \rho^{*}\left(\tilde{A}_{0}\right) y_{10}=y_{1 r}$. Since $\delta \chi\left(\tilde{A}_{0}\right)=-2, \operatorname{tr}_{V_{x 10}^{*}} \tilde{A}_{c}=10=\operatorname{dim}$ $V_{x_{10}}^{*}$, we have $\operatorname{ord}_{\Lambda_{10}} f^{s}=-2 s-\frac{10}{2}$. By Corollary 1-2, we have $b_{\Lambda_{10}}(s) / b_{\Lambda_{1}}(s)$ $=(s+5)$.

(4) The isotropy subalgebra $g_{x_{27}}$ is g. Put $y_{27}=x_{0}$ and $y_{27}^{\prime}=x_{1}^{\prime}$. Then $d \rho\left(\tilde{A}_{0}\right) x_{27}=0$ and $d \rho^{*}\left(\tilde{A}_{0}\right) y_{27}=y_{27}$ for $\tilde{A}_{0}=R_{-I_{3}}$. Since $\delta \chi\left(\tilde{A}_{0}\right)=-3, \operatorname{tr}_{V_{x_{27}}^{*}} \tilde{A}_{0}$ 
$=\operatorname{dim} V_{x_{27}}^{*}=27$, we have $\operatorname{ord}_{\Lambda_{27}} f^{s}=-3 s-\frac{27}{2}$. Since $\Lambda_{10}=\Lambda_{1}^{*}, \Lambda_{27}=\Lambda_{0}^{*}$, we have codim $A_{10} \cap A_{27}=1$ and $b_{\Lambda_{27}}(s) / b_{\Lambda_{10}}(s)=(s+9)$. Thus we obtain the $b$ function $b(s)=(s+1)(s+5)(s+9)$ and the holonomy diagram (Figure 6-1). Note that the relative invariant $f(X)$ is given by the determinant $\operatorname{det} X$ of $X$ in $\mathscr{J}$.

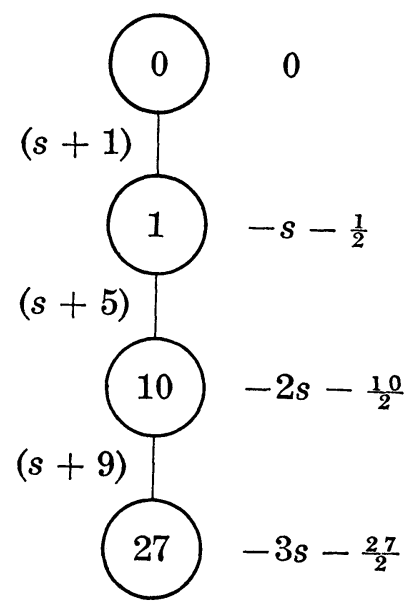

Figure 6-1. Holonomy diagram of $\left(G L(1) \times E_{6}\right.$, $\left.\square \otimes \Lambda_{1}, V(1) \otimes V(27)\right)$

\section{§ 7. $\left(G L(1) \times E_{7}, \square \otimes \Lambda_{6}, V(1) \otimes V(56)\right)$}

The representation space $V(1) \otimes V(56)$ is identified with

$$
V=\left\{X=\left(x, x^{\prime}\right) ; x, x^{\prime} \in M(8),{ }^{t} x=-x,{ }^{t} x^{\prime}=-x^{\prime}\right\} .
$$

Then the infinitesimal action $d \rho$ of $\mathfrak{g}=\mathfrak{g r}(1) \oplus E_{7}$ is given by

$$
\begin{aligned}
& \text { (1) } \quad\left(x, x^{\prime}\right) \stackrel{p}{\mapsto}\left(p x+x^{t} p,-{ }^{t} p y-y p\right) \text { for } p \in S L(8, C) \\
& \text { (2) }\left(x, x^{\prime}\right) \stackrel{c}{\mapsto}\left(c x, c x^{\prime}\right) \text { for } \quad c \in g^{\gamma}(1) \\
& \text { (3) }\left(\left(x_{i j}\right),\left(x_{i j}^{\prime}\right)\right) \stackrel{\circ}{\mapsto}\left(\left(\sum_{m, n=1}^{8} \vartheta^{i j m n} y_{m n}\right), \quad\left(-\sum_{m, n=1}^{8} \vartheta_{i j m n} x_{m n}\right)\right)
\end{aligned}
$$

where $\vartheta$ denotes a tensor, antisymmetric in its indices, and upper, lower indices satisfy the relation

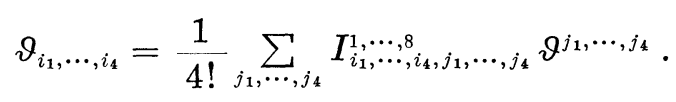




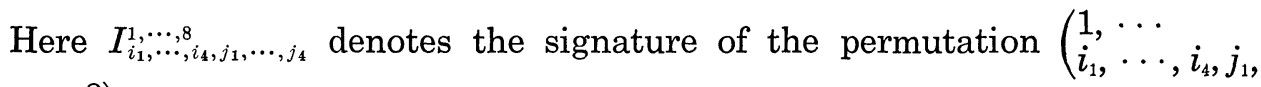
$\cdots, 8)$ when $\left\{i_{1}, \cdots, i_{4}, j_{1}, \cdots, j_{4}\right\}=\{1, \cdots, 8\}$, and 0 otherwise. The product as a Lie algebra is given as follows:

(1) $\left[p, p^{\prime}\right]=p p^{\prime}-p^{\prime} p$ where $p p^{\prime}$ denotes the matrix multiplication

(2) $[p, \vartheta]=\vartheta^{\prime}$ where $\left(\vartheta^{\prime}\right)^{i j k \ell}=\sum_{m}\left(\vartheta^{m j k l} p_{i m}+\vartheta^{i m k \ell} p_{j m}+\vartheta^{i j m \ell} p_{k m}+\right.$ $\left.\vartheta^{i j k m} p_{\ell m}\right)$

(3) $\left[\vartheta, \vartheta^{\prime}\right]=p$ where $p_{i j}=\frac{2}{3}\left(\sum_{\ell, m, n}\left(\vartheta^{\ell m n i}\left(\vartheta^{\prime}\right)_{\ell m n j}-\frac{1}{8}\left(\sum_{r} \vartheta^{\ell m n r}\left(\vartheta^{\prime}\right)_{\ell m n r}\right) \delta_{i j}\right)\right.$

Proposition 7-1 (Stephen J. Haris). There exist five orbits $S_{m}=\rho(G) x_{m}$ ( $m=0,1,11,28,56)$ where $S_{m}$ denotes the m-codimensional $G$-orbit and $x_{m}$ is given as follows.
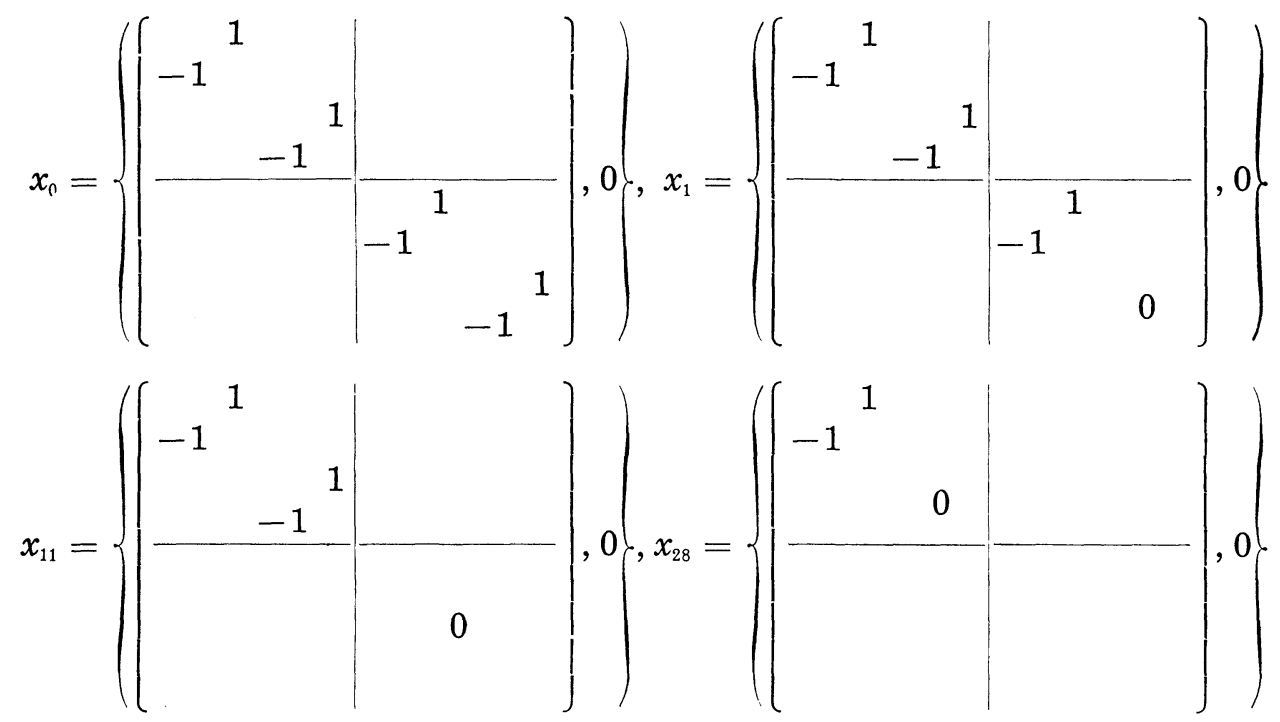

and $x_{56}=(0,0)$.

Proof. See [5].

Q.E.D.

We identify the dual vector space $V^{*}$ with $V$ by $\langle X, Y\rangle=\operatorname{tr} x x^{\prime}+$ $\operatorname{tr} y y^{\prime}$ for $X=\left(x, x^{\prime}\right), \quad Y=\left(y, y^{\prime}\right) \in V$.

Then the dual action $d \rho^{*}$ is given as follows:

(1) $\left(y, y^{\prime}\right) \stackrel{p^{*}}{\mapsto}\left(-{ }^{t} p y-y p, p y^{\prime}+y^{t} p\right)$

(2) $\quad\left(y, y^{\prime}\right) \stackrel{c^{*}}{\mapsto}\left(-c y,-c y^{\prime}\right)$

(3) $\quad\left(\left(y_{i j}\right),\left(y_{i j}^{\prime}\right)\right) \stackrel{\theta^{*}}{\mapsto}\left(\left(\sum_{m, n=1}^{8} \vartheta_{i j m n} y_{m n}^{\prime}\right),\left(-\sum_{m, n=1}^{8} \vartheta^{i j m n} y_{m n}\right)\right)$ 
Since $G=G L(1) \times E_{7}$ is reductive, the dual triplet $\left(G, \rho^{*}, V^{*}\right)$ has also five orbits $S_{m}^{*}(m=0,1,11,28,56)$. We denote by $\Lambda_{m}$ (resp. $\left.\Lambda_{m}^{*}\right)$ the conormal bundle of $S_{m}$ (resp. $S_{m}^{*}$ ).

(1) The isotropy subalgebra $g_{x_{0}}$ at $x_{0}$ is the Lie algebra of $E_{6}$ (See [5], [1]). Since $\Lambda_{0}=V \times\{0\}=\Lambda_{66}^{*}$, we have $\operatorname{ord}_{\Lambda_{0}} s^{s}=0$ where $f(X)=\operatorname{Pf}(x)+$ $\operatorname{Pf}\left(x^{\prime}\right)-\frac{1}{4} \operatorname{tr}\left(x x^{\prime} x x^{\prime}\right)+\frac{1}{16} \operatorname{tr}\left(x x^{\prime}\right)^{2}$ for $X=\left(x, x^{\prime}\right) \in V$.

(2) The isotropy subalgebra $g_{x_{1}}$ at $x_{1}$ is the set $\{c \oplus p \oplus \theta\}$ satisfying the following conditions:

$$
p=\left(\begin{array}{cc}
-\frac{c}{2} I_{6} & \\
& \frac{3}{2} c I_{2}
\end{array}\right)+\left(\begin{array}{llll}
p_{1} & p_{12} & p_{13} & p_{14} \\
p_{12}^{\prime} & p_{2} & p_{23} & p_{24} \\
p_{13}^{\prime} & p_{23}^{\prime} & p_{3} & p_{34} \\
0 & 0 & 0 & p_{4}
\end{array}\right) \text { where } \operatorname{tr} p_{i}=0
$$

for $i=1, \cdots, 4$, and $p_{i j} p_{i j}^{\prime}=-\operatorname{det} p_{i j} \cdot I_{2}$ for $1 \leq i<j \leq 3 . \quad \vartheta=\left(\vartheta_{i j k \ell}\right)$ satisfies $\vartheta_{12 i j}+\vartheta_{34 i j}+\vartheta_{56 i j}=0$.

In fact, the isotropy subgroup is connected, and is isomorphic to $\left(G L(1) \times F_{4}\right) \cdot U$ where $U$ is unipotent of dimension 26 (See [5]). The conormal vector space $V_{x_{1}}^{*}$ is given by

$$
V_{x_{1}}^{*}=\left\{x=\left(\left(\begin{array}{r|rr}
0 & 0 & 0 \\
\hline 0 & 0 & x \\
-x & 0
\end{array}\right), 0\right) ; x \in C\right\} .
$$

Let $y_{1}$ be the element of $V_{x_{1}}^{*}$ with $x=1$ in (7.5). Then it is a generic point, and $y_{1}^{\prime}=0$ is the point of the one-codimensional orbit. Let $A_{0}$ be an element of $g_{x_{1}}$ with $c=-\frac{1}{4}$, all remaining parts zero in (7.4). Then $d \rho\left(A_{0}\right) x_{1}=0$ and $d \rho^{*}\left(A_{0}\right) y_{1}=y_{1}$. Since $\delta \chi\left(A_{0}\right)=-1, \operatorname{tr}_{V_{x_{1}}^{*}} A_{0}=\operatorname{dim} V_{x_{1}}^{*}=1$, we have $\operatorname{ord}_{\Lambda_{1}} f^{s}=-s-\frac{1}{2}$ and $b_{\Lambda_{1}}(s) / b_{\Lambda_{0}}(s)=(s+1)$.

(3) The isotropy subalgebra $g_{x_{11}}$ at $x_{11}$ is the set $\{c \oplus p \oplus \theta\}$ satisfying the following conditions

$$
\begin{aligned}
& p=\left(\begin{array}{c|c}
-\frac{c}{2} I_{4}+p_{1} & p_{2} \\
\hline 0 & \frac{c}{2} I_{4}+p_{4}
\end{array}\right) \quad \text { where } K^{-1} p_{1} K \in \mathfrak{S p}_{\mathfrak{p}}(2) \text { with } \\
& K=\left[\begin{array}{llll}
1 & & & \\
& 0 & 1 & \\
& 1 & 0 & \\
& & & 1
\end{array}\right], \operatorname{tr} p_{4}=0,(\vartheta) \text { with } \vartheta_{12 i j}+\vartheta_{34 i j}=0 \text { for all } i, j .
\end{aligned}
$$

The conormal vector space $V_{x_{11}}^{*}$ is given by 


$$
V_{x_{11}}^{*}=\left\{\tilde{Y}=\left(\left(\begin{array}{cc}
0 & 0 \\
0 & Y_{4}
\end{array}\right),\left(\begin{array}{cc}
Y_{1}^{\prime} & 0 \\
0 & 0
\end{array}\right)\right) ; y_{12}^{\prime}+y_{34}^{\prime}=0\right\} .
$$

Then, for $A=c \oplus p \oplus \theta$ in $\mathrm{g}_{x_{11}}$, we have $d \rho^{*}(A) \tilde{Y}=\left(\left(\begin{array}{cc}0 & 0 \\ 0 & Z_{4}\end{array}\right),\left(\begin{array}{cc}Z_{1}^{\prime} & 0 \\ 0 & 0\end{array}\right)\right)$ where

$$
Z_{4}=-2 c Y_{4}-{ }^{t} P_{4} Y_{4}-Y_{4} P_{4}+\left(\sum_{m, n=1}^{4} \vartheta_{i j m n} y_{m n}^{\prime}\right)
$$

and

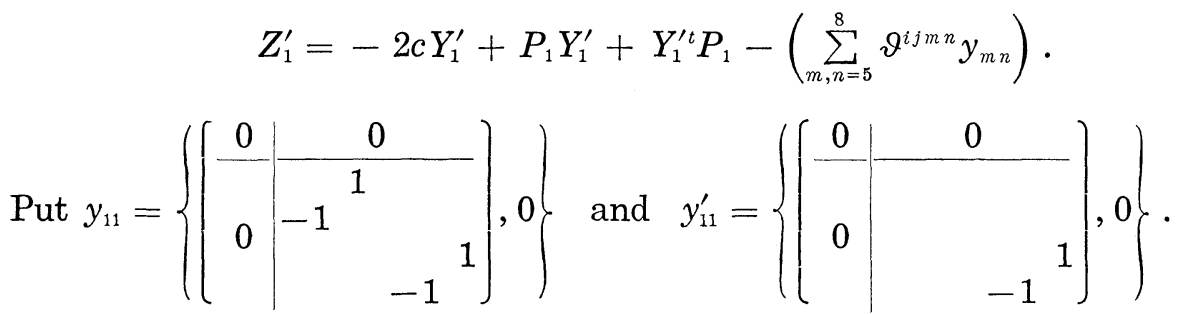

Then $y_{11}$ is a generic point and $y_{11}^{\prime}$ is a point of the unique onecodimensional orbit. Thus we have $\Lambda_{11}=\Lambda_{11}^{*}$ and $\operatorname{dim} \Lambda_{1} \cap \Lambda_{11}=\operatorname{dim} V-1$. Let $A_{0}$ be an element of $g_{x_{11}}$ with $c=-\frac{1}{2}$, all remaining parts zero in (7.7). Then $d \rho\left(A_{0}\right) x_{11}=0$ and $d \rho^{*}\left(A_{0}\right) y_{11}=y_{11}$. Since $\delta \chi\left(A_{0}\right)=4 c=-2, \operatorname{tr}_{V_{x_{11}^{*}}^{*}} A_{0}$ $=-22 c=11$ and $\operatorname{dim} V_{x_{11}}^{*}=11$, we have $\operatorname{ord}_{A_{11}} f^{s}=-2 s-\frac{11}{2}$ and hence $b_{A_{11}}(s) / b_{A_{1}}(s)=\left(s+\frac{11}{2}\right)$.

(4) The isotropy subalgebra $\mathfrak{g}_{x_{28}}$ at $x_{28}$ is the set $\{c \oplus p \oplus \theta\}$ satisfying the following conditions:

$$
\begin{aligned}
& p=\left(\begin{array}{c|c}
-\frac{c}{2} I_{2}+p_{1} & p_{2} \\
\hline 0 & \frac{c}{6} I_{2}+p_{4}
\end{array}\right] \text { with } \operatorname{tr} p_{1}=\operatorname{tr} p_{4}=0 \\
& \vartheta=\left(\vartheta^{i j k c}\right) \text { with } \vartheta_{12 i j}=0 \text { for all } i, j .
\end{aligned}
$$

The conormal vector space $V_{x_{28}}^{*}$ is given by

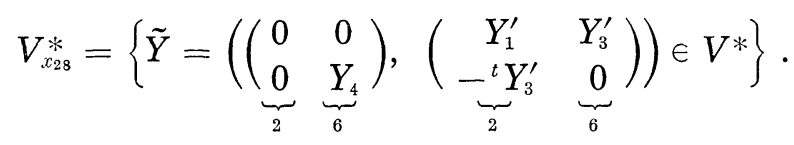

Then for $A=c \oplus p \oplus \theta$ in (7.8), we have

$$
d \rho^{*}(A) \tilde{Y}=\left(\left(\begin{array}{cc}
0 & 0 \\
0 & Z_{4}
\end{array}\right),\left(\begin{array}{cc}
Z_{1}^{\prime} & Z_{3}^{\prime} \\
-{ }^{t} Z_{3}^{\prime} & 0
\end{array}\right)\right)
$$


where

$$
\begin{aligned}
& Z_{4}=-\frac{4}{3} c Y_{4}-{ }^{t} p_{4} Y_{4}-Y_{4} p_{4}+\left(\sum_{m, n} \vartheta_{i j m n} y_{m n}^{\prime}\right) \\
& Z_{1}^{\prime}=-2 c Y_{1}^{\prime}+p_{1} Y_{1}^{\prime}+Y_{1}^{\prime t} p_{1}-p_{2}^{t} Y_{3}^{\prime}+Y_{3}^{\prime t} p_{2}-\left(\sum_{m, n=3}^{8} \vartheta^{i j m n} y_{m n}\right) \\
& Z_{3}^{\prime}=-\frac{4}{3} c Y_{3}^{\prime}+p_{1} Y_{3}^{\prime}+Y_{3}^{\prime t} p_{4}-\left(\sum_{m, n=3}^{8} \vartheta^{i j m n} y_{m n}\right)
\end{aligned}
$$

Therefore, one can see that the colocalization at $x_{28}$ has at most unique one-codimensional orbit.

Since $\Lambda_{28}=\Lambda_{1}^{*}$ and $\Lambda_{11}=\Lambda_{11}^{*}, \Lambda_{28}$ is a good holonomic variety and $\operatorname{dim} \Lambda_{28} \cap \Lambda_{11}=\operatorname{dim} V-1$.

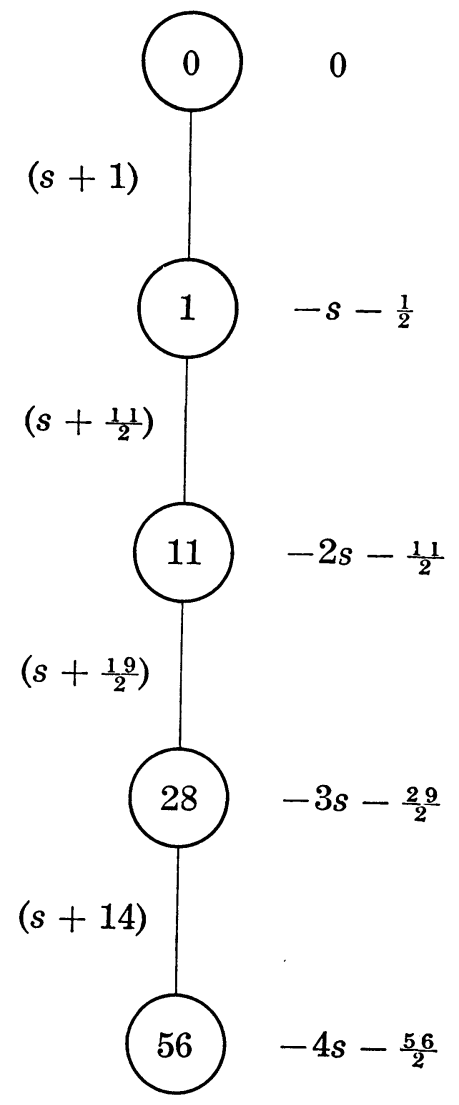

Figure 7-1. Holonomy diagram of $\left(G L(1)_{4}^{*} \times E_{7}\right.$, $\left.\square \otimes \Lambda_{1}, V(1) \otimes V(56)\right)$ 


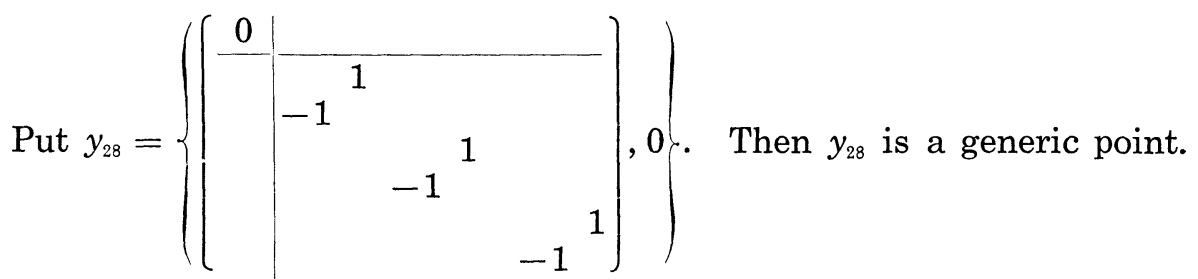

Let $A_{0}$ be an element of $\mathfrak{g}_{x_{28}}$ with $c=-\frac{3}{4}$, all remaining parts zero in (7.8). Then we have $d \rho\left(A_{0}\right) x_{28}=0$ and $d \rho^{*}\left(A_{0}\right) y_{28}=y_{28}$. Since $\delta \chi\left(A_{0}\right)=4 c$ $=-3, \operatorname{tr}_{V_{x_{28}}^{*}} A_{0}=-38 c=\frac{57}{2}$ and $\operatorname{dim} V_{x_{28}}^{*}=28$, we have ord ${ }_{\Lambda_{28}} f^{s}=-3 s-\frac{29}{2}$ and hence $b_{\Lambda_{28}}(s) / b_{\Lambda_{11}}(s)=\left(s+\frac{19}{2}\right)$ by Corollary 1-2.

(5) Since $x_{56}=0$, we have $\left(G_{x_{56}}, \rho_{x_{56}}, V_{x_{58}}^{*}\right)=\left(G, \rho^{*}, V^{*}\right)$. Since $\Lambda_{56}=$ $\{0\} \times V^{*}=\Lambda_{0}^{*}$ and $\Lambda_{28}=\Lambda_{1}^{*}$, we have $\operatorname{dim} \Lambda_{56} \cap \Lambda_{28}=\operatorname{dim} V-1$ and they intersect regularly. Let $A_{0}$ be an element of $g_{x_{28}}$ with $c=-1$, all remaining parts zero in (7.2). Then $d \rho\left(A_{0}\right) x_{56}=0$ and $d \rho^{*}\left(A_{0}\right) y_{56}=y_{56}$ where $y_{56}=x_{0}$. Since $\delta \chi\left(A_{0}\right)=-4, \operatorname{tr}_{V^{x_{56}}}^{*} A_{0}=\operatorname{dim} V_{x_{56}}^{m}=56$, we have $\operatorname{ord}_{4_{56}} s^{s}=$ $-4 s-\frac{56}{2}$, and hence $b_{\Lambda_{58}}(s) / b_{\Lambda_{28}}(s)=s+14$. Thus we obtain the $b$-function $b(s)=(s+1)\left(s+\frac{11}{2}\right)\left(s+\frac{19}{2}\right)(s+14)$ and the holonomy diagram (Figure 7-1).

\section{§ 8. ( $\left(G L(6), \Lambda_{3}, V(20)\right)$}

Let $V_{1}$ be a 6 -dimensional vector space spanned by $u_{1}, \cdots, u_{6}$. Then $G=G L(6)$ acts on $V_{1}$ by $\rho_{1}(g)\left(u_{1}, \cdots, u_{6}\right)=\left(u_{1}, \cdots, u_{6}\right) g$ for $g \in G$. The representation space $V=V(20)$ is spanned by skew-tensors $u_{i} \wedge u_{j} \wedge u_{k}$ $(1 \leq i<j<k \leq 6)$, and $\rho=\Lambda_{3}$ is given by $\rho(g)\left(u_{i} \wedge u_{j} \wedge u_{k}\right)=\rho_{1}(g) u_{i} \wedge$ $\rho_{1}(g) u_{j} \wedge \rho_{1}(g) u_{k}$ for $1 \leq i<j<k \leq 6$, and $g \in G$. Then it is well-known (and also one can easily check) that there exist five $G$-orbits $S_{m}=\rho(G) x_{m}$ $(m=0,1,5,10,20)$ where $S_{m}$ denotes the $m$-codimensional orbit, and $x_{0}=$ $u_{1} \wedge u_{2} \wedge u_{3}+u_{4} \wedge u_{5} \wedge u_{6}, x_{1}=u_{1} \wedge u_{2} \wedge u_{3}+u_{1} \wedge u_{4} \wedge u_{5}+u_{2} \wedge u_{4} \wedge u_{6}$, $x_{5}=u_{1} \wedge u_{2} \wedge u_{3}+u_{1} \wedge u_{4} \wedge u_{5}, x_{10}=u_{1} \wedge u_{2} \wedge u_{3}$, and $x_{20}=0$. We identify the dual space $V^{*}$ with $V$ by $\left(\sum a_{i j k} u_{i} \wedge u_{j} \wedge u_{k}, \sum b_{r s t} u_{r} \wedge u_{s} \wedge u_{t}\right)=$ $\sum_{1 \leq i<j<k \leq 6} a_{i j k} b_{i j k}$. Since $(G, \rho, V) \cong\left(G, \rho^{*}, V^{*}\right)$, there exist also five orbits $S_{m}^{*}(m=0,1,5,10,20)$ in $V^{*}$. We denote by $\Lambda_{m}$ the conormal bundle of $S_{m}$. The isotropy subalgebra $\mathfrak{g}_{x}$ at $x \in V(20)$ is, by definition, $\mathfrak{g}_{x}=\{A \in \mathfrak{g} \mathfrak{l}(6)$; $d \rho(A) x=0\}$ where $d \rho(A)\left(u_{i} \wedge u_{j} \wedge u_{k}\right)=d \rho_{1}(A) u_{i} \wedge u_{j} \wedge u_{k}+u_{i} \wedge d \rho_{1}(A) u_{j}$ $\wedge u_{k}+u_{i} \wedge u_{j} \wedge d \rho_{1}(A) u_{k}$.

(1) The isotropy subalgebra $\mathfrak{g}_{x_{0}}$ is, by simple calculation, given as follows: 


$$
\mathfrak{g}_{x_{0}}=\left\{\left(\begin{array}{cc}
A & 0 \\
0 & B
\end{array}\right) \in \mathfrak{g r}(6) ; A, B \in \mathfrak{g r}(3)\right\} .
$$

We have $\Lambda_{0}=V \times\{0\}$, and hence $\operatorname{ord}_{\Lambda_{0}} f^{s}=0$ where $f$ denotes the relatively invariant irreducible polynomial of degree four (See [1], [14]).

(2) Put $x_{1}^{\prime}=u_{1} \wedge u_{2} \wedge u_{6}+u_{2} \wedge u_{3} \wedge u_{4}-u_{1} \wedge u_{3} \wedge u_{5}$. Then $x_{1}^{\prime} \in S_{1}$. By simple calculation, the isotropy subalgebra $g_{x_{1}^{\prime}}$ at $x_{1}^{\prime}$ is given by

$$
\mathfrak{g}_{x_{1}^{\prime}}=\left\{\tilde{A}=\left(\frac{A}{0} \mid \frac{B}{A-(\operatorname{tr} A) \cdot I_{3}}\right) \in \mathfrak{g}((6) ; A, B \in M(3), \operatorname{tr} B=0\} .\right.
$$

Therefore we have $G_{x_{1}^{\prime}} \sim G L(3) \cdot\left(G_{a}\right)^{8}$ where $\cdot$ denotes a semi-direct product and $G_{a} \cong C$. The conormal vector space $V_{x_{1}}^{*}$ is of one-dimension with a basis $u_{4} \wedge u_{5} \wedge u_{6}$. The action $d \rho_{x_{1}^{\prime}}$ of $g_{x_{1}^{\prime}}$ on $V_{x_{1}^{\prime}}^{*}$ is $d \rho_{x_{1}^{\prime}}(\tilde{A}) u_{4} \wedge u_{5} \wedge u_{6}$ $=-2 \operatorname{tr} \tilde{A} \cdot u_{4} \wedge u_{5} \wedge u_{6}$. Take $A_{0} \in \mathfrak{g}_{x_{1}^{\prime}}$ with $\operatorname{tr} A_{0}=-\frac{1}{2}$. Then we have $d \rho\left(A_{0}\right) x_{1}^{\prime}=0$ and $d \rho^{*}\left(A_{0}\right) y_{1}=y_{1}$ where $y_{1}=u_{4} \wedge u_{5} \wedge u_{6}$. Since $\delta \chi\left(A_{0}\right)=$ $(\operatorname{deg} f / \operatorname{dim} V) \operatorname{tr} d \rho\left(A_{0}\right)=\frac{4}{20} \times\left(10 \operatorname{tr} A_{0}\right)=-1$ and $\operatorname{tr}_{V x_{1}^{\prime}}^{*} A_{0}=\operatorname{dim} V_{x_{1}^{\prime}}^{*}=1$, we have $\operatorname{ord}_{1_{1}} f^{s}=-s-\frac{1}{2}$ by Proposition 1-3. Since 0 is the point of the one-codimensional orbit, we have $\operatorname{dim} \Lambda_{0} \cap \Lambda_{1}=\operatorname{dim} V-1$ and $\Lambda_{0} \cap \Lambda_{1}$ is $G_{0}$-prehomogeneous, i.e., $\Lambda_{1}$ is a good holonomic variety by Proposition 1-5. Also we have $\mu=1$ and $\nu=0$ by Proposition 1-4, i.e., $\Lambda_{0}$ and $\Lambda_{1}$ intersect regularly. By Corollary $1-2$, we have $b_{\Lambda_{1}}(s) / b_{\Lambda_{0}}(s)=(s+1)$.

(3) Put $x_{5}^{\prime}=u_{1} \wedge\left(u_{2} \wedge u_{4}+u_{3} \wedge u_{5}\right) \in S_{5}$. Then the isotropy subalgebra $\mathfrak{g}_{x^{\prime}}$ is given as follows:

$$
\begin{aligned}
\mathfrak{g}_{x_{5}} & =\left\{\left(\begin{array}{c|c|c}
-2 \varepsilon & B & C \\
\hline 0 & A+\varepsilon I_{4} & D \\
\hline 0 & 0 & \eta
\end{array}\right) \in \mathfrak{g} \mathfrak{l}(6) ; A \in \mathfrak{S} \mathfrak{p}(2),{ }^{t} B, D \in C^{4}, C \in C\right\} \\
& =\left(\mathfrak{g} p_{\mathfrak{p}}(2) \oplus \mathfrak{g} \mathfrak{g}(1) \oplus \mathfrak{g} \mathfrak{l}(1)\right) \oplus \mathfrak{H}(9)
\end{aligned}
$$

where $u(9)$ denotes the Lie algebra of 9-dimensional unipotent group. Put $\omega_{1}=\left(u_{2} \wedge u_{4}-u_{3} \wedge u_{5}\right) \wedge u_{6}, \omega_{2}=u_{4} \wedge u_{5} \wedge u_{6}, \omega_{3}=u_{3} \wedge u_{4} \wedge u_{6}, \omega_{4}$ $=u_{2} \wedge u_{5} \wedge u_{6}$ and $\omega_{5}=u_{2} \wedge u_{3} \wedge u_{6}$. Then the conormal vector space $V_{x_{5^{\prime}}}^{*}$ is spanned by $\omega_{1}, \cdots, \omega_{5}$ and $\left(G_{x_{5}}, \rho_{x_{5}}, V_{x_{5}}^{*}\right) \cong\left(G L(1) \times S p(2), \Lambda_{1} \otimes \Lambda_{2}, V(1)\right.$ $\otimes V(5)) \cong\left(G L(1) \times S O(5), \Lambda_{1} \otimes \Lambda_{1}, V(1) \otimes V(5)\right)$, where $\omega_{1}$ is a generic point and $\omega_{2}=u_{4} \wedge u_{5} \wedge u_{6}$ is a point of the one-codimensional orbit. Therefore we have $\operatorname{dim} \Lambda_{1} \cap \Lambda_{5}=\operatorname{dim} V-1$. Since the $\left(G_{x_{5}^{\prime}} \cap G_{0}\right)$-orbit of $\omega_{2}$ is onecodimensional in $V_{x_{5}}^{*}$, i.e., $\Lambda_{1} \cap \Lambda_{5}$ is $G_{0}$-prehomogeneous, $\Lambda_{5}$ is a good holonomic variety by (2) and Proposition 1-5. Let $A_{0}$ be an element of $\mathfrak{g}_{x_{5}}$ with $\eta=-1$ and all remaining parts zero in (8.3). Then we have 
$d \rho\left(A_{0}\right) x_{5}^{\prime}=0$ and $d \rho^{*}\left(A_{0}\right) \omega_{1}=\omega_{1}$. Since $\delta \chi\left(A_{0}\right)=2 \operatorname{tr} A_{0}=-2, \operatorname{tr}_{V x_{5}^{\prime}} A_{0}=$ $-5(2 \varepsilon+\eta)=5$, and $\operatorname{dim} V_{x_{5^{\prime}}}^{*}=5$, we have $\operatorname{ord}_{\Lambda_{5}} f^{s}=-2 s-\frac{5}{2}$. Put $A_{\beta}=$ $\beta\left(E_{22}-E_{44}\right)+(\beta+1) E_{66}$ for $\beta \in C$ where $E_{i j}$ denotes the matrix unit. Then $d \rho\left(A_{\beta}\right) x_{5}^{\prime}=0$ and $d \rho^{*}\left(A_{\beta}\right) \omega_{2}=\omega_{2}$. Since $\tilde{V}=V_{x^{\prime}}^{*} \bmod d \rho_{x_{5}^{\prime}}\left(\mathfrak{g}_{x_{5}^{\prime}}\right) \omega_{2}$ is spanned by $u_{2} \wedge u_{3} \wedge u_{6}$, we have $\operatorname{tr}_{\tilde{v}} A_{\beta}=2 \beta+1$. Hence we have $\mu=1$ and $\nu=0$ by Proposition 1-4, i.e., $\Lambda_{1}$ and $\Lambda_{5}$ intersect regularly. One can also get this from the fact $m_{A_{5}}-m_{A_{1}}=1$. By Corollary 1-2, we have $b_{A_{5}}(s) / b_{A_{1}}(s)$ $=s+\frac{5}{2}$.

(4) Put $x_{10}=u_{1} \wedge u_{2} \wedge u_{3} \in S_{10}$. Then the isotropy subalgebra $\mathfrak{g}_{x_{10}}$ is given as follows:

$$
\begin{aligned}
\mathfrak{g}_{x_{10}}= & \left\{\tilde{A}=\left(\frac{A}{0} \mid \frac{B}{C}\right) \in \mathfrak{g l}(6) ; A, B, C \in M(3), \operatorname{tr} A=0\right\} \\
& \cong(\mathfrak{g l}(3) \oplus \mathfrak{g l}(3)) \oplus V(9), \text { i.e., } G_{x_{10}} \sim(S L(3) \times G L(3)) \cdot\left(G_{a}\right)^{9}
\end{aligned}
$$

In general, we write $G_{1} \sim G_{2}$ when two groups $G_{1}$ and $G_{2}$ are locally isomorphic to each other. Put $\omega_{1}=u_{1} \wedge u_{4} \wedge u_{5}, \omega_{2}=u_{1} \wedge u_{4} \wedge u_{6}, \omega_{3}=$ $u_{1} \wedge u_{5} \wedge u_{6}, \omega_{4}=u_{2} \wedge u_{4} \wedge u_{5}, \omega_{5}=u_{2} \wedge u_{4} \wedge u_{6}, \omega_{6}=u_{2} \wedge u_{5} \wedge u_{6}, \omega_{7}=$ $u_{3} \wedge u_{4} \wedge u_{5}, \omega_{8}=u_{3} \wedge u_{4} \wedge u_{6}, \omega_{9}=u_{3} \wedge u_{5} \wedge u_{6}$ and $\omega_{10}=u_{4} \wedge u_{5} \wedge u_{6}$. Then the conormal vector space $V_{x_{10}}^{*}$ is spanned by $\omega_{1}, \cdots, \omega_{10}$. The action $d \rho_{x_{10}}$ of $\mathfrak{g}_{x_{10}}$ on $V_{x_{10}}^{*}$ is given by

$$
d \rho_{x_{10}}(\tilde{A})\left(\omega_{1}, \cdots, \omega_{10}\right)=\left(\omega_{1}, \cdots, \omega_{10}\right)\left(\begin{array}{c:c}
d \tilde{\rho}(A \oplus C) & 0 \\
\hline B^{\prime} & -\operatorname{tr} A
\end{array}\right)\left({ }^{t} B^{\prime} \in C^{9}\right)
$$

where $\tilde{\rho}=\Lambda_{2} \otimes \Lambda_{2}^{*}$, i.e., the action of $G_{x_{0}}$ induced on the subspace spanned by $\omega_{1}, \cdots, \omega_{9}$ is isomorphic to a triplet $\left(S L(3) \times G L(3), \Lambda_{1} \otimes \Lambda_{1}, V(3) \otimes V(3)\right)$ as a triplet (See [1]). Then $\omega_{1}+\omega_{5}+\omega_{9} \in S_{1}^{*}$ is a generic point and $\omega_{1}$ $+\omega_{5} \in S_{5}^{*}$ is a point of the one-codimensional orbit. This implies that $\operatorname{dim} \Lambda_{5} \cap \Lambda_{10}=\operatorname{dim} V-1$. Since $\Lambda_{5} \cap \Lambda_{10}$ is $G_{0}$-prehomogeneous, $\Lambda_{10}$ is a good holonomic variety by Proposition 1-5. Let $\tilde{A}$ be an element of $g_{x_{10}}$ with $A=B=0$ and $C=-\frac{1}{2} I_{3}$ in (8.4). Then $d \rho(\tilde{A}) x_{10}=0$ and $d \rho^{*}(\tilde{A})$ $\left(\omega_{1}+\omega_{5}+\omega_{9}\right)=\left(\omega_{1}+\omega_{5}+\omega_{9}\right)$. Since $\delta \chi(\tilde{A})=2 \cdot \operatorname{tr} \tilde{A}=-3, \operatorname{tr}_{1}{ }_{v_{10}} \tilde{A}=$ $-7 \operatorname{tr} C=\frac{21}{2}$ and $\operatorname{dim} V_{x_{10}}^{*}=10$, we have $\operatorname{ord}_{\Lambda_{10}} f^{s}=-3 s-\frac{11}{2}$ by Proposition 1-3. Let $A_{\beta}$ be an element of $g_{x_{10}}$ with $A=B=0$ and $C=\left(\begin{array}{cc}1-(\beta / 2) & 0 \\ 0 & (\beta / 2) I_{2}\end{array}\right)$ in (8.4). Then $d_{\rho} \rho\left(A_{\beta}\right) x_{10}=0$ and $d \rho\left(A_{\beta}\right)\left(\omega_{1}+\omega_{5}\right)=\left(\omega_{1}+\omega_{5}\right)$. Since $\tilde{V}=$ $V_{x_{10}}^{*} \bmod d \rho_{x_{10}}\left(g_{x_{10}}\right)\left(\omega_{1}+\omega_{5}\right)$ is spanned by $u_{3} \wedge u_{5} \wedge u_{6}$, we have $\operatorname{tr}_{\tilde{v}} A_{\beta}=\beta$. This implies that $\mu=1$ and $\nu=0$, i.e., $\Lambda_{5}$ and $\Lambda_{10}$ intersect regularly by Proposition 1-4. One can also get this from the fact $m_{\Lambda_{10}}-m_{A_{5}}=1$. By 
Corollary 1-2, we have $b_{\Lambda_{10}}(s) / b_{\Lambda_{5}}(s)=s+\frac{7}{2}$.

(5) Put $x_{20}=0 \in S_{20}$. In this case, $\left(G_{x_{20}}, \rho_{x_{20}}, V_{x_{20}}^{*}\right) \cong\left(G L(6), \Lambda_{3}, V(20)\right)$. $A_{x_{20}}=\{0\} \times V^{*}$ is a good holonomic variety. Put $\tilde{A}=-\frac{1}{3} I_{6}$. Then $d \rho(\tilde{A}) x_{20}$ $=0$ and $d \rho^{*}(\tilde{A}) x_{0}^{*}=x_{0}^{*} \quad$ where $x_{0}^{*}=u_{1} \wedge u_{2} \wedge u_{3}+u_{4} \wedge u_{5} \wedge u_{6} \in S_{0}^{*}$. Since $\delta \chi(\tilde{A})=2 \operatorname{tr} \tilde{A}=-4, \operatorname{tr}_{V_{x_{20}}^{*}} \tilde{A}=20$ and $\operatorname{dim} V_{x_{20}}^{*}=20$, we have $\operatorname{ord}_{\Lambda_{20}} f^{s}$ $=-4 s-\frac{20}{2}$ by Proposition 1-3. Put $A_{\beta}=\left(\begin{array}{lll}a_{1} & & 0 \\ & \ddots & \\ 0 & & a_{6}\end{array}\right)$ with $a_{1}=a_{2}=a_{4}$ $=1 / 2-\beta / 6, a_{3}=a_{5}=a_{6}=\beta / 3$. Then $d \rho\left(A_{\beta}\right) x_{20}=0$ and $d \rho^{*}\left(A_{\beta}\right) x_{1}^{*}=x_{1}^{*}$ where $x_{1}^{*}=u_{1} \wedge u_{2} \wedge u_{3}+u_{1} \wedge u_{4} \wedge u_{5}+u_{2} \wedge u_{4} \wedge u_{6}$. Since $\tilde{V}=V_{x_{20}}^{*}$ $\bmod d \rho_{x_{20}}\left(g_{x_{20}}\right) x_{1}^{*}$ is spanned by $u_{3} \wedge u_{5} \wedge u_{6}$, we have $\operatorname{tr}_{\tilde{V}} A_{\beta}=\beta$. This implies that $\mu=1$ and $\nu=0$, i.e., $\Lambda_{20}$ and $\Lambda_{10}$ intersect regularly. One can also get this from $m_{\Lambda_{20}}-m_{\Lambda_{10}}=1$. By Corollary 1-2, we have $b_{\Lambda_{20}}(s) / b_{\Lambda_{10}}(s)$ $=s+5$. Thus we obtain the $b$-function $b(s)=(s+1)\left(s+\frac{5}{2}\right)\left(s+\frac{7}{2}\right)(s+5)$ and the holonomy diagram (Figure $8-1$ ). We denote $\Lambda_{m}$ by $m$.

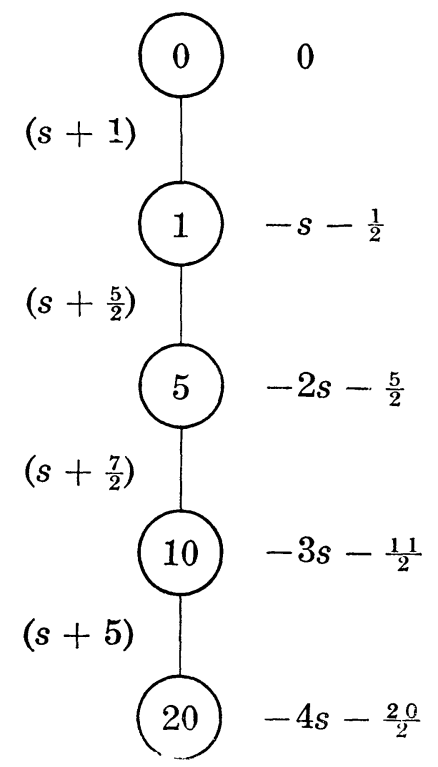

Figure 8-1. Holonomy diagram of $\left(G L(6), \Lambda_{3}, V(20)\right)$.

§ 9. $\left(G L(1) \times S p(3), \square \otimes \Lambda_{3}, V(1) \otimes V(14)\right)$

Put $\omega_{1}=u_{1} \wedge u_{2} \wedge u_{3}, \omega_{2}=u_{4} \wedge u_{5} \wedge u_{6}, \omega_{3}=u_{2} \wedge u_{3} \wedge u_{4}, \omega_{4}=u_{1} \wedge$ $u_{5} \wedge u_{6}, \omega_{5}=u_{1} \wedge u_{3} \wedge u_{5}, \omega_{6}=u_{2} \wedge u_{4} \wedge u_{6}, \omega_{7}=u_{1} \wedge u_{2} \wedge u_{6}, \omega_{8}=u_{3} \wedge$ $u_{4} \wedge u_{5}, \omega_{9}=u_{1} \wedge u_{2} \wedge u_{5}-u_{1} \wedge u_{3} \wedge u_{6}, \omega_{10}=u_{2} \wedge u_{4} \wedge u_{5}-u_{3} \wedge u_{4} \wedge u_{6}$, 
$\omega_{11}=u_{1} \wedge u_{2} \wedge u_{4}+u_{2} \wedge u_{3} \wedge u_{6}, \omega_{12}=u_{1} \wedge u_{4} \wedge u_{5}+u_{3} \wedge u_{5} \wedge u_{6}, \omega_{13}=$ $u_{1} \wedge u_{3} \wedge u_{4}-u_{2} \wedge u_{3} \wedge u_{5}, \omega_{14}=u_{1} \wedge u_{4} \wedge u_{6}-u_{2} \wedge u_{5} \wedge u_{6}$. Then the representation space $V$ is identified with the subspace of $V(20)$ in $\S 8$ generated by $\omega_{1}, \cdots, \omega_{14}$. Then the representation $\rho=\square \otimes \Lambda_{3}$ is the restriction of $\Lambda_{3}$ for $G L(6)$ to $G=G L(1) \times S p(3)$. The orbital decomposition of this space has been completed by J-I. Igusa (See [3]). There exist five $G$-orbits $S_{m}=\rho(G) x_{m}(m=0,1,4,7,14)$ where $S_{m}$ denotes the $m$-codimensional orbit, and $x_{0}=\omega_{1}+\omega_{2}, x_{1}=\omega_{7}+\omega_{13}, x_{4}=\omega_{13}, x_{7}=\omega_{1}, x_{14}=0$. We identify the dual space $V^{*}$ with $V$ by $\left(\sum_{i=1}^{14} a_{i} \omega_{i}, \sum_{j=1}^{14} b_{j} \omega_{j}\right)=\sum_{k=1}^{14} a_{k} b_{k}$. Since $(G, \rho, V) \cong\left(G, \rho^{*}, V^{*}\right)$, there exist also five $G$-orbits $S_{m}^{*}(m=0,1,4$, $7,14)$ in $V^{*}$. We denote by $\Lambda_{m}$ the conormal bundle of $S_{m}$. The Lie algebra $g$ of $G=G L(1) \times S p(3)$ is given as follows:

$$
\mathfrak{g}=\left\{\tilde{A}=(d) \oplus\left(\begin{array}{cc}
A & B \\
C & -{ }^{t} A
\end{array}\right) ; A, B, C \in M(3),{ }^{t} B=B,{ }^{t} C=C\right\}
$$

(1) Since $d \rho(\tilde{A}) x_{0}=(d+\operatorname{tr} A) \omega_{1}+(d-\operatorname{tr} A) \omega_{2}+c_{1} \omega_{3}+b_{1} \omega_{4}-c_{2} \omega_{5}-$ $b_{2} \omega_{6}+c_{3} \omega_{7}+b_{3} \omega_{8}+c_{23} \omega_{9}+b_{23} \omega_{10}+c_{13} \omega_{11}+b_{13} \omega_{12}-c_{12} \omega_{13}-b_{12} \omega_{14}$ where $c_{i}=$ $c_{i i}$ and $b_{i}=b_{i i}$ for $i=1,2,3$, we have

$$
\mathfrak{g}_{x_{0}}=\left\{\tilde{A}=(0) \oplus\left(\begin{array}{cc}
A & 0 \\
0 & -{ }^{t} A
\end{array}\right) ; A \in \mathfrak{g l}(3)\right\} \cong \mathfrak{g l}(3) \text {. }
$$

We have $\Lambda_{0}=V \times\{0\}$, and hence $\operatorname{ord}_{\Lambda_{0}} f^{s}=0$ where $f$ denotes the relatively invariant irreducible polynomial of degree four (See [1], [3]).

(2) Since $d \rho(\tilde{A}) x_{1}=\left(b_{3}-2 b_{12}\right) \omega_{1}+2 a_{21} \omega_{3}+c_{2} \omega_{4}-2 a_{12} \omega_{5}-c_{1} \omega_{6}+(d+$ $\left.a_{1}+a_{2}-a_{3}\right) \omega_{7}+2 c_{12} \omega_{8}+\left(a_{13}-a_{32}\right) \omega_{9}-c_{13} \omega_{10}+\left(a_{23}-a_{31}\right) \omega_{11}-c_{23} \omega_{12}+(d+$ $\left.a_{3}\right) \omega_{13}+\left(c_{12}-c_{3}\right) \omega_{14}$ where $a_{i}=a_{i i}$ for $i=1,2,3$, we have

$$
\begin{aligned}
\mathfrak{g}_{x_{1}}= & \left\{(d) \oplus\left[\begin{array}{ccc|ccc}
-d+\alpha & 0 & \beta & b_{1} & b_{12} & b_{13} \\
0 & -d-\alpha & \gamma & b_{12} & b_{2} & b_{23} \\
\gamma & \beta & -d & b_{13} & b_{23} & 2 b_{12} \\
\hline & 0 & & d-\alpha & 0 & -\gamma \\
0 & d+\alpha & -\beta \\
& & & -\beta & -\gamma & d
\end{array}\right]\right\} \\
& \cong(\mathfrak{g l}(1) \oplus \mathfrak{o}(3)) \oplus V(5)
\end{aligned}
$$

where $V(5)$ denotes the Lie algebra of $\left(G_{a}\right)^{5}$.

The conormal vector space $V_{x_{1}}^{*}$ is one-dimensional with a basis $\omega_{2}$. The action $d \rho_{x_{1}}$ of $g_{x_{1}}$ on $V_{x_{1}}^{*}$ is given by $d \rho_{x_{1}}(\tilde{A}) \omega_{2}=\left(-d+a_{1}+a_{2}+a_{3}\right) \omega_{2}$ $=-4 d \omega_{2}$. Therefore we have $\Lambda_{1}=\overline{G\left(x_{1}, y_{1}\right)}$ where $y_{1}=\omega_{2}$. Let $A_{0}$ be an 
element of $g_{x_{1}}$ with $d=-\frac{1}{4}$, all remaining parts zero in (9.3). Then we have $d \rho\left(A_{0}\right) x_{1}=0$ and $d \rho^{*}\left(A_{0}\right) y_{1}=y_{1}$. Since $\delta \chi\left(A_{0}\right)=4 d=-1, \operatorname{tr}_{V_{x_{1}}^{*}} \tilde{A}=$ $\operatorname{dim} V_{x_{1}}^{*}=1$, we have $\operatorname{ord}_{\Lambda_{1}} f^{s}=-s-\frac{1}{2}$ by Proposition 1-3. Since 0 is the point of the one-codimensional orbit, we have $\operatorname{dim} \Lambda_{0} \cap \Lambda_{1}=\operatorname{dim} V-1$ and $\Lambda_{0} \cap \Lambda_{1}$ is $G_{0}$-prehomogeneous, i.e., $\Lambda_{1}$ is a good holonomic variety by Proposition 1-5. Also we have $\mu=1$ and $\nu=0$ by Proposition 1-4, i.e., $\Lambda_{0}$ and $\Lambda_{1}$ intersect regularly. By Corollary $1-2$, we have $b_{\Lambda_{1}}(s) / b_{\Lambda_{0}}(s)=$ $(s+1)$.

(3) Since $d \rho(\tilde{A}) x_{4}=-2 b_{12} \omega_{1}+2 a_{21} \omega_{3}-2 a_{12} \omega_{5}+2 c_{12} \omega_{8}+a_{13} \omega_{9}-c_{13} \omega_{10}$ $+a_{23} \omega_{11}-c_{23} \omega_{12}+\left(d+a_{3}\right) \omega_{13}-c_{3} \omega_{14}$, we have

$$
\mathfrak{g}_{x_{4}}=\left\{(d) \oplus\left\{\begin{array}{ccc|ccr}
a_{1} & 0 & 0 & b_{1} & 0 & \beta \\
0 & a_{2} & 0 & 0 & b_{2} & \delta \\
\alpha & \gamma & -d & \beta & \delta & \varepsilon \\
\hline c_{1} & 0 & 0 & -a_{1} & 0 & -\alpha \\
0 & c_{2} & 0 & 0 & -a_{2} & -\gamma \\
0 & 0 & 0 & 0 & 0 & d
\end{array}\right)\right\}
$$

$$
\begin{aligned}
& \cong\left\{(d) \oplus\left(\begin{array}{c|cc|cc|r}
-d & \alpha & \beta & \gamma & \delta & \varepsilon \\
\hline 0 & a_{1} & b_{1} & 0 & \beta \\
c_{1} & -a_{1} & \multicolumn{1}{|c|}{0} & -\alpha \\
\hline 0 & 0 & a_{2} & b_{2} & \delta \\
\hline 0 & 0 & c_{2} & -a_{2} & -\gamma \\
\hline 0 & 0 & 0 & d
\end{array}\right]\right\} \\
& \simeq(g r(1) \oplus \operatorname{gr}(2) \oplus \mathfrak{g r}(2)) \oplus V(5) .
\end{aligned}
$$

The conormal vector space $V_{x_{4}}^{*}$ is spanned by $\omega_{2}, \omega_{4}, \omega_{6}, \omega_{7}$ on which $\mathfrak{g}_{x_{4}}$ acts as follows:

$$
\left(\omega_{2}, \omega_{4}, \omega_{6}, \omega_{7}\right) \mapsto\left(\omega_{2}, \omega_{4}, \omega_{6}, \omega_{7}\right)\left(\begin{array}{rrrr}
A_{1} & -b_{1} & b_{2} & 0 \\
-c_{1} & A_{2} & 0 & -b_{2} \\
c_{2} & 0 & A_{3} & b_{1} \\
0 & -c_{2} & c_{1} & A_{4}
\end{array}\right)
$$

where $A_{1}=a_{1}+a_{2}-2 d, A_{2}=-a_{1}+a_{2}-2 d, A_{3}=a_{1}-a_{2}-2 d, A_{4}=$ $-a_{1}-a_{2}-2 d$.

Hence we have $\left(G_{x_{4}}, \rho_{x_{4}}, V_{x_{4}}^{*}\right) \cong\left(S L(2) \times G L(2), \Lambda_{1} \otimes \Lambda_{1}, V(2) \otimes V(2)\right) \cong$ $\left(G L(1) \times S O(4), \square \otimes \Lambda_{1}, V(1) \otimes V(4)\right)$.

Clearly, $y_{4}=\omega_{4}+\omega_{6}$ is its generic point, and $\omega_{2}$ is a point of the one-codimensional orbit. Since $\Lambda_{1}=G\left(x_{1}, \omega_{2}\right)$, we have $\operatorname{dim} \Lambda_{1} \cap \Lambda_{4}=\operatorname{dim}$ $V$ - 1. Since $\Lambda_{1} \cap \Lambda_{4}$ is $G_{0}$-prehomogeneous, $\Lambda_{4}$ is a good holonomic variety by (2) and Proposition 1-5. Let $A_{0}$ be an element of $\mathfrak{g}_{x_{4}}$ with $d$ 
$=-\frac{1}{2}$ and all remaining parts zero in (9.4). Then $d \rho\left(A_{0}\right) x_{4}=0$ and $d \rho^{*}\left(A_{0}\right) y_{4}=y_{4}$. Since $\delta \chi\left(A_{0}\right)=4 d=-2, \operatorname{tr}_{V_{x_{4}}^{*}} A_{0}=-8 d=4$ and $\operatorname{dim} V_{x_{4}}^{*}$ $=4$, we have $\operatorname{ord}_{A_{1}} f^{s}=-2 s-\frac{4}{2}$. Let $A_{\beta}$ be an element of $g_{x_{4}}$ with $a_{1}=$ $\frac{1}{2}(1-\beta), d=-\frac{1}{4}(\beta+1)$, all remaining parts zero in (9.4). Then we have $d \rho\left(A_{\beta}\right) x_{4}=0, d \rho^{*}\left(A_{\beta}\right) \omega_{2}=\omega_{2}$ and $\operatorname{tr}_{\tilde{V}} A_{\beta}=\beta$ where $\tilde{V}=V_{x_{4}}^{*} \bmod d \rho_{x_{4}}\left(\mathfrak{g}_{x_{4}}\right) \omega_{2}$. This implies that $\Lambda_{4}$ and $\Lambda_{1}$ intersect regularly by Proposition 1-4. By Corollary 1-2, we have $b_{A_{4}}(s) / b_{A_{1}}(s)=(s+2)$.

(4) Since $d \rho(\tilde{A}) x_{7}=\left(d+a_{1}+a_{2}+a_{3}\right) \omega_{1}+c_{1} \omega_{3}-c_{2} \omega_{5}+c_{3} \omega_{7}+c_{23} \omega_{9}+$ $c_{13} \omega_{11}-c_{12} \omega_{13}$, we have

$$
\mathfrak{g}_{x_{7}}=\left\{\tilde{A}=(-\operatorname{tr} A) \oplus\left(\frac{A}{0} \mid \frac{B}{-{ }^{t} A}\right) ;{ }^{t} B=B\right\} \simeq \mathfrak{g r}(3) \oplus V(6) .
$$

The conormal vector space $V_{x_{7}}^{*}$ is spanned by $\omega_{2}, \omega_{4}, \omega_{6}, \omega_{8}, \omega_{10}, \omega_{12}, \omega_{14}$, and $\mathfrak{g}_{x_{\tau}}$ acts on $V_{x_{7}}^{*}$ as follows:

$$
d \rho_{x_{7}}(\tilde{A})\left(\omega_{2}, \omega_{4}, \cdots, \omega_{14}\right)=\left(\omega_{2}, \omega_{4}, \cdots, \omega_{14}\right)\left(\frac{2 \operatorname{tr} A}{0} \mid \frac{B}{2 \operatorname{tr} A \cdot I_{6} \oplus d \rho_{1}^{*}(A)}\right)
$$

where ${ }^{t} B \in C^{6}$ and $\rho_{1}=2 \Lambda_{1}$.

Then $y_{7}=\omega_{4}+\omega_{10}$ is its generic point, and $\omega_{4}+\omega_{6}$ is a point of the one-codimensional orbit. Since $\Lambda_{4}=\overline{G\left(x_{4}, \omega_{4}+\omega_{6}\right)}$, we have $\operatorname{dim} \Lambda_{4} \cap \Lambda_{7}=$ $\operatorname{dim} V-1$. Since $\Lambda_{4} \cap \Lambda_{7}$ is $G_{0}$-prehomogeneous, $\Lambda_{7}$ is a good holonomic variety by (3) and Proposition 1-5. Let $A_{0}$ be an element of $\mathrm{g}_{x_{7}}$ with $A$ $=\frac{1}{4} I_{3}$ and $B=0$ in (9.5). Then $d \rho\left(A_{0}\right) x_{7}=0$ and $d \rho^{*}\left(A_{0}\right) y_{7}=y_{7}$. Since $\delta \chi\left(A_{0}\right)=-4 \operatorname{tr} A=-3, \operatorname{tr}_{V_{x}}^{*} A_{0}=10 \operatorname{tr} A=\frac{15}{2}$ and $\operatorname{dim} V_{x_{7}}^{*}=7$, we have $\operatorname{ord}_{A_{7}} f^{s}=-3 s-\frac{8}{2}$. Let $A_{\beta}$ be an element of $\mathfrak{g}_{x_{7}}$ with $a_{1}=a_{2}=\frac{\beta}{4}, a_{3}=\frac{1}{2}$ $-\frac{\beta}{4}$, all remaining parts zero in (9.5). Then $d \rho\left(A_{\beta}\right) x_{7}=0, d \rho\left(A_{\beta}\right)\left(\omega_{4}+\omega_{6}\right)$ $=\left(\omega_{4}+\omega_{6}\right)$ and $\operatorname{tr}_{\tilde{V}} A_{\beta}=\beta$ where $\tilde{V}=V_{x_{7}}^{*} \bmod d \rho_{x_{7}}\left(\mathfrak{g}_{x_{7}}\right)\left(\omega_{4}+\omega_{6}\right)=C \omega_{8}$. This implies that $\Lambda_{4}$ and $\Lambda_{7}$ intersect regularly by Proposition 1-4. By Corollary $1-2$, we have $b_{A_{7}}(s) / b_{A_{4}}(s)=\left(s+\frac{5}{2}\right)$.

(5) Since $x_{14}=0$, we have $\left(G_{x_{14}}, \rho_{x_{14}}, V_{x_{14}}^{*}\right) \cong\left(G L(1) \times S p(3), \square \otimes \Lambda_{3}\right.$, $V(1) \otimes V(14))$ and $\Lambda_{14}=\{0\} \times V^{*}$ is a good holonomic variety. Take $\tilde{A}=$ $(-1) \oplus(0) \in \mathfrak{g}=\mathfrak{g r}(1) \oplus \mathfrak{z h}(3)$. Then $d \rho(\tilde{A}) x_{14}=0, d \rho^{*}(\tilde{A})\left(\omega_{1}+\omega_{2}\right)=\left(\omega_{1}+\omega_{2}\right)$. Since $\delta \chi(\widetilde{A})=-4, \operatorname{tr}_{V x_{14}}^{*} \tilde{A}=14$ and $\operatorname{dim} V_{x_{14}}^{*}=14$, we have $\operatorname{ord}_{1_{14}} f^{s}=-4 s$ - $\frac{14}{2}$. Since $\Lambda_{14}=\Lambda_{0}^{*}, \Lambda_{7}=\Lambda_{1}^{*}$ where $\Lambda_{m}^{*}$ denotes the conormal bundle of $S_{m}^{*}\left(\subset V^{*}\right)$, they intersect regularly by (2). Note that $(G, \rho, V) \cong\left(G, \rho^{*}, V^{*}\right)$ since $G=G L(1) \times S p(3)$ is reductive. By Corollary $1-2$, we have $b_{A_{14}}(s) /$ $b_{A_{7}}(s)=s+\frac{7}{2}$. Since $b_{A_{14}}(s)=b(s)$ and $b_{A_{0}}(s)=1$, we obtain the $b$-function 


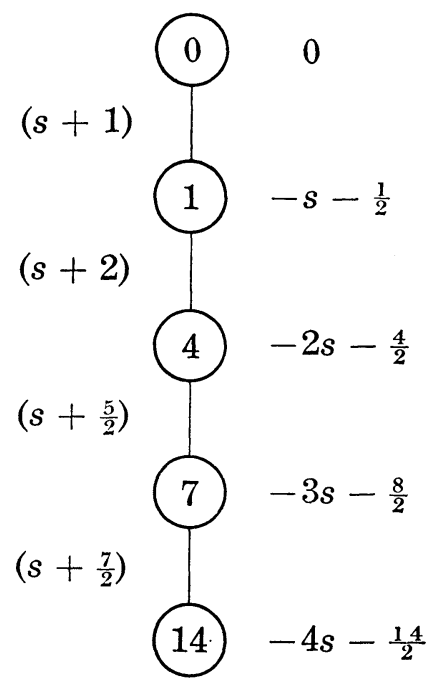

Figure 9-1. Holonomy diagram of $(G L(1) \times S p(3)$, $\left.\square \otimes A_{3}, V(1) \otimes V(14)\right)$.

$b(s)=(s+1)(s+2)\left(s+\frac{5}{2}\right)\left(s+\frac{7}{2}\right)$ and the holonomy diagram (Figure 9-1).

We denote $\Lambda_{m}$ by $m$.

\section{$\S 10 . \quad\left(G L(7), \Lambda_{3}, V(35)\right)$}

The representation space $V=V(35)$ is spanned by the skew-tensors $u_{i} \wedge u_{j} \wedge u_{k}(1 \leq i<j<k \leq 7)$ of degree three, on which $G=G L(7)$ acts as in $\S 8$. Then it is known (See [6], [7]) that there exist ten orbits $S_{m}$ $=\rho(G) x_{m}(m=0,1,4,7,9,10,14,15,22,35)$, where $S_{m}$ denotes the $m$-codimensional orbit, and $x_{0}=u_{2} \wedge u_{3} \wedge u_{4}+u_{5} \wedge u_{6} \wedge u_{7}+u_{1} \wedge\left(u_{2} \wedge u_{5}+u_{3}\right.$ $\left.\wedge u_{6}+u_{4} \wedge u_{7}\right), x_{1}=u_{2} \wedge u_{3} \wedge u_{5}+u_{3} \wedge u_{4} \wedge u_{6}+u_{1} \wedge\left(u_{2} \wedge u_{7}-u_{4} \wedge u_{5}\right)$, $x_{4}=u_{1} \wedge u_{3} \wedge u_{4}+u_{2} \wedge u_{5} \wedge u_{6}+u_{1} \wedge u_{2} \wedge u_{7}, x_{7}=u_{2} \wedge u_{3} \wedge u_{4}+u_{1} \wedge$ $\left(u_{2} \wedge u_{5}+u_{3} \wedge u_{6}+u_{4} \wedge u_{7}\right), x_{9}=u_{1} \wedge u_{2} \wedge u_{3}+u_{4} \wedge u_{5} \wedge u_{6}, x_{10}=u_{1} \wedge$ $u_{2} \wedge u_{6}-u_{1} \wedge u_{3} \wedge u_{5}+u_{2} \wedge u_{3} \wedge u_{4}, x_{14}=u_{1} \wedge\left(u_{2} \wedge u_{5}+u_{3} \wedge u_{6}+u_{4} \wedge\right.$ $\left.u_{7}\right), x_{15}=u_{1} \wedge\left(u_{2} \wedge u_{4}+u_{3} \wedge u_{5}\right), x_{22}=u_{1} \wedge u_{2} \wedge u_{3}$ and $x_{35}=0$. Note that we chose these representative points $x_{m}$ so that the isotropy subalgebra $g_{x_{m}}$ at $x_{m}$ will be the standard form. The relative invariant $f(x)$ of this space is of degree seven (See [1], [14]). Since $(G, \rho, V) \cong\left(G, \rho^{*}, V^{*}\right)$, there exist also ten $G$-orbits $S_{m}^{*}(m=0,1,4,7,9,10,14,15,22,35)$ in $V^{*}$. We denote by $\Lambda_{m}$ (resp. $\Lambda_{m}^{*}$ ) the conormal bundle of $S_{m}$ (resp. $S_{m}^{*}$ ). Clearly we have $\Lambda_{0}=V \times\{0\}=\Lambda_{35}^{*}$ and $\Lambda_{35}=\{0\} \times V^{*}=\Lambda_{0}^{*}$. 
(1) The isotropy subalgebra $\mathfrak{g}_{x_{0}}$ at $x_{0}$ is given as follows (See [1]).

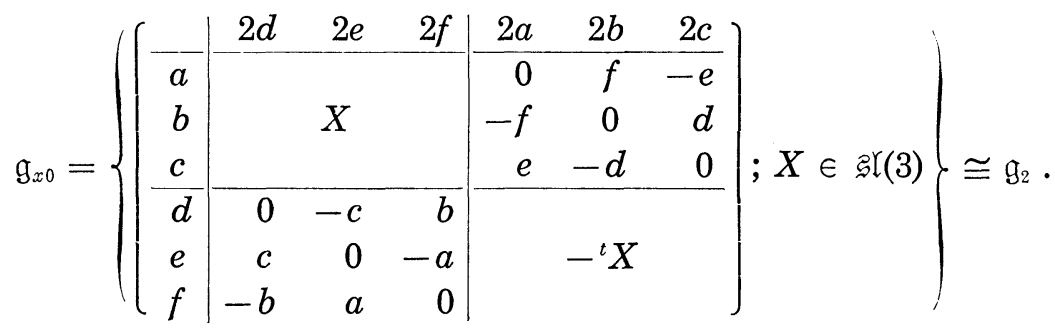

Since $\Lambda_{0}=V \times\{0\}$, we have ord $f_{0} f^{s}=0$.

(2) The isotropy subalgebra $g_{x_{1}}$ at $x_{1}$ is given as follows.

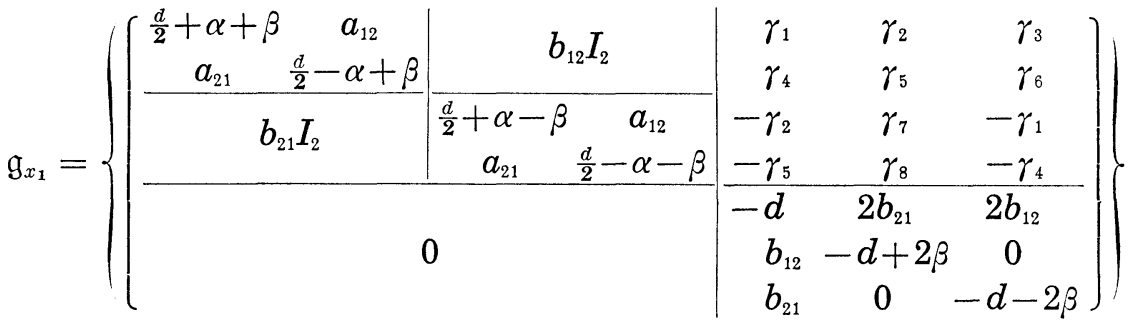

$$
\begin{aligned}
& \cong(\mathfrak{g l}(1) \oplus \mathfrak{g l}(2) \oplus \mathfrak{g} l(2)) \oplus V(8) .
\end{aligned}
$$

The conormal vector space $V_{x_{1}}^{*}$ is spanned by $u_{5} \wedge u_{6} \wedge u_{7}$. Then $d \rho_{x_{1}}(A) u_{5} \wedge u_{6} \wedge u_{7}=3 d u_{5} \wedge u_{6} \wedge u_{7}$ for $A \in g_{x_{1}}$. Since 0 is the point of the one-codimensional $G$-orbit, $\Lambda_{0}$ and $\Lambda_{1}$ intersect regularly with codimension one. Let $A_{0}$ be an element of $\mathfrak{g}_{x_{1}}$ with $d=\frac{1}{3}$, all remaining parts zero in (10.2). Then $d \rho\left(A_{0}\right) x_{1}=0$ and $d \rho^{*}\left(A_{0}\right) y_{1}=y_{1}$ where $y_{1}=u_{5} \wedge u_{6} \wedge u_{7}$. Since $\delta \chi\left(A_{0}\right)=(\operatorname{deg} f / \operatorname{dim} V) \cdot \operatorname{tr}_{V} A_{0}=3 \operatorname{tr} A_{0}=-3 d=-1$ (See Proposition 1-9), $\operatorname{tr}_{V_{x_{1}}^{*}} A_{0}=\operatorname{dim} V_{x_{1}}^{*}=1$, we have $\operatorname{ord}_{\Lambda_{1}} f^{s}=-s-\frac{1}{2}$ and hence $b_{\Lambda_{1}}(s) / b_{\Lambda_{0}}(s)=$ $(s+1)$. We have also $\Lambda_{1}=\Lambda_{22}^{*}$, and hence $\Lambda_{22}=\Lambda_{1}^{*}$.

(3) The isotropy subalgebra $\mathfrak{g}_{x_{4}}$ at $x_{4}$ is given as follows.

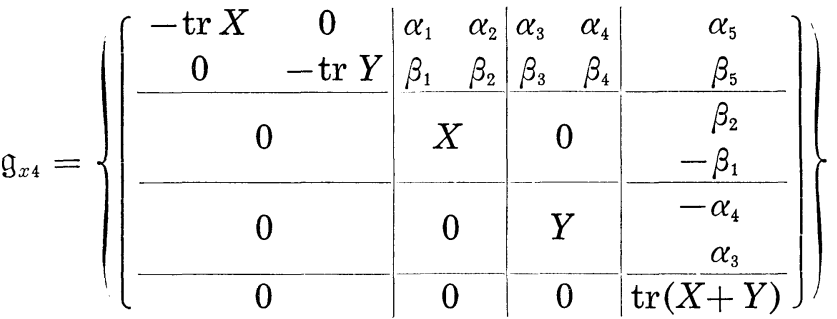

$$
\begin{aligned}
& \cong(\mathfrak{g l}(2) \oplus \mathfrak{g l}(2)) \cdot \mathfrak{u}(10) \text {. }
\end{aligned}
$$

The conormal vector space $V_{\tilde{K}_{4}}^{*}$ is spanned by $\omega_{1}=u_{3} \wedge u_{5} \wedge u_{7}, \omega_{2}=u_{3}$ 
$\wedge u_{6} \wedge u_{7}, \omega_{3}=u_{4} \wedge u_{5} \wedge u_{7}, \omega_{4}=u_{4} \wedge u_{6} \wedge u_{7}$. Then we have $\left(G_{x_{4}}, \rho_{x_{4}}\right.$, $V_{x_{4}}^{*} \cong\left(G L(2) \times G L(2), \Lambda_{1} \otimes \Lambda_{1}, V(2) \otimes V(2)\right)$, and $y_{4}=\omega_{1}+\omega_{4}$ is its generic point, $y_{4}^{\prime}=\omega_{1}$ is a point of the one-codimensional orbit. Since the colocalization $\left(G_{x_{4}}, \rho_{x_{4}}, V_{x_{4}}^{*}\right)$ is an irreducible regular P.V., $\Lambda_{4}$ is a good holonomic variety by Corollary 1-8. Let $A_{0}$ be an element of $\mathrm{g}_{x_{4}}$ with $X=-\frac{1}{3} I_{2}$, all remaining parts zero in (10.3). Then $d \rho\left(A_{0}\right) x_{4}=0$ and $d_{\rho} *\left(A_{0}\right) y_{4}=y_{4}$. Since $\delta \chi\left(A_{0}\right)=3 \operatorname{tr} A_{0}=-2, \operatorname{tr}_{v_{x_{4}}^{*}} A_{0}=4 \operatorname{dim} V_{x_{4}}^{*}$, we have ord $\operatorname{or}_{A_{4}} f^{s}=-2 s-$ $\frac{4}{2}$ by Proposition 1-3. We have also $d \rho^{*}\left(A_{0}\right) y_{4}^{\prime}=y_{4}^{\prime}$ and $\operatorname{tr}_{\tilde{V}} A_{0}=1$ where $\tilde{V}=V_{x_{4}}^{*} \bmod d \rho_{x_{4}}\left(\mathrm{~g}_{x_{4}}\right) y_{4}^{\prime}=C \omega_{4}$. This implies that $\Lambda_{1}$ and $\Lambda_{4}$ intersect regularly with codimension one by Proposition 1-4. By Corollary 1-2, we have $b_{A_{4}}(s) / b_{\Lambda_{1}}(s)=(s+2)$. We have also $\Lambda_{4}=\Lambda_{15}^{*}$ and hence $\Lambda_{15}=\Lambda_{4}^{*}$.

(4) The isotropy subalgebra $\mathfrak{g}_{x_{7}}$ at $x_{7}$ is given as follows.

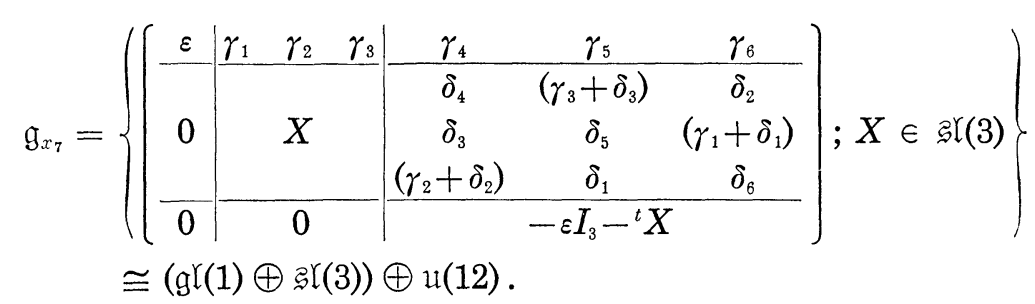

Put $\omega_{1}=u_{5} \wedge u_{6} \wedge u_{7}, \omega_{2}=u_{2} \wedge u_{6} \wedge u_{7}, \omega_{3}=u_{4} \wedge u_{5} \wedge u_{6}, \omega_{4}=u_{3} \wedge u_{5} \wedge$ $u_{7}, \omega_{5}=u_{2} \wedge u_{5} \wedge u_{7}-u_{3} \wedge u_{6} \wedge u_{7}, \omega_{6}=u_{4} \wedge u_{5} \wedge u_{7}-u_{3} \wedge u_{5} \wedge u_{6}, \omega_{7}$ $=u_{2} \wedge u_{5} \wedge u_{6}+u_{4} \wedge u_{6} \wedge u_{7^{7}}$. Then the conormal vector space $V_{x_{7}}^{*}$ is spanned by these $\omega_{1}, \cdots, \omega_{7}$. The action $d \rho_{x_{7}}$ of $\mathfrak{g}_{x_{7}}$ on $V_{x_{7}}^{*}$ is given by

$$
d \rho_{x_{7}}(A)\left(\omega_{1}, \cdots, \omega_{7}\right)=\left(\omega_{1}, \cdots, \omega_{7}\right)\left(\begin{array}{c|c|c}
3 \varepsilon & * * * * * * \\
\hline 0 & 2 \varepsilon I_{6} \oplus d \rho_{1}^{*}(X)
\end{array}\right)
$$

where $\rho_{1}=2 \Lambda_{1}$ for $S L(3)$.

Here $y_{7}=\omega_{2}+\omega_{3}+\omega_{4}$ is its generic point, and $y_{7}^{\prime}=\omega_{2}+\omega_{3}$ is a point of the one-codimensional orbit. This implies that $\Lambda_{7}=\Lambda_{10}^{*}, \Lambda_{10}=\Lambda_{7}^{*}$ and $\operatorname{dim} \Lambda_{4} \cap \Lambda_{7}=\operatorname{dim} V-1$. Since $\Lambda_{4} \cap \Lambda_{7}$ is $G_{0}$-prehomogeneous, $\Lambda_{7}$ is a good holonomic variety. Let $A_{0}$ be an element of $\mathfrak{g}_{x_{7}}$ with $\varepsilon=\frac{1}{2}$, all remaining parts zero in (10.4). Then $d \rho\left(A_{0}\right) x_{7}=0$ and $d_{\rho} *\left(A_{0}\right) y_{7}=y_{7}$. Since $\delta \chi\left(A_{0}\right)=3 \operatorname{tr} A_{0}=-6 \varepsilon=-3, \operatorname{tr}_{V_{x_{7}}^{*}} A_{0}=\frac{15}{2}$ and $\operatorname{dim} V_{x_{7}}^{*}=7$, we have $\operatorname{ord}_{A_{7}} f^{s}=-3 s-\frac{8}{2}$ by Proposition 1-3. Let $A_{\beta}$ be an element of $\mathrm{g}_{x_{7}}$ with $\varepsilon=\frac{\beta}{6}+\frac{1}{3}, X=\left(\begin{array}{ccc}\eta & & \\ & \eta & \\ & & -2 \eta\end{array}\right)$ with $\eta=\frac{\beta}{6}-\frac{1}{6}$, all remaining parts zero in (10.4). Then we have $d \rho\left(A_{\beta}\right) x_{7}=0, d \rho\left(A_{\beta}\right) y_{7}^{\prime}=y_{7}^{\prime}$ and $\operatorname{tr}_{\tilde{v}} A_{\beta}=\beta$ where $\tilde{V}=V_{x_{7}}^{*} \bmod d \rho_{x_{7}}\left(\mathrm{~g}_{x_{7}}\right) y_{7}^{\prime}=C \omega_{4}$. This implies that $\Lambda_{4}$ and $\Lambda_{7}$ intersect regularly 
by Proposition 1-4. By Corollary 1-2, we have $b_{A_{7}}(s) / b_{A_{4}}(s)=\left(s+\frac{5}{2}\right)$.

(5) The isotropy subalgebra $\mathfrak{g}_{x_{9}}$ at $x_{9}$ is given as follows.

$$
\begin{aligned}
\mathfrak{g}_{x_{9}}= & \left\{\left(\begin{array}{ccc}
X & 0 & Z \\
0 & Y & W \\
0 & 0 & \varepsilon
\end{array}\right) ; X, Y \in \mathfrak{g l}(3), Z, W \in C^{3}\right\} \\
& \cong(\mathfrak{g l}(1) \oplus \mathfrak{g l}(3) \oplus \mathfrak{g l}(3)) \oplus V(6) .
\end{aligned}
$$

The conormal vector space $V_{x_{9}}^{*}$ is spanned by $u_{i} \wedge u_{j} \wedge u_{7}(1 \leq i \leq 3 ; 4 \leq$ $j \leq 6)$. By seeing the weights, we have $\left(G_{x_{9}}, \rho_{x_{9}}, V_{x_{9}}^{*}\right) \cong\left(S L(3) \times G L(3), \Lambda_{1}\right.$ $\left.\otimes \Lambda_{1}, V(3) \otimes V(3)\right)$. Since this is an irreducible regular P.V., $\Lambda_{9}$ is a good holonomic variety by Corollary 1-8. As a generic point, we may take $y_{9}$ $=\left(u_{1} \wedge u_{4}+u_{2} \wedge u_{5}+u_{3} \wedge u_{6}\right) \wedge u_{7}$, and $y_{9}^{\prime}=\left(u_{1} \wedge u_{4}+u_{2} \wedge u_{5}\right) \wedge u_{7}$ is a point of the one-codimensional orbit. This implies that $\Lambda_{9}=\Lambda_{14}^{*}, \Lambda_{14}=\Lambda_{9}^{*}$ and $\operatorname{dim} \Lambda_{4} \cap \Lambda_{9}=\operatorname{dim} V-1$. Let $A_{0}$ be an element of $\mathfrak{g}_{x_{9}}$ with $\varepsilon=-1$, all remaining parts zero in (10.6). Then $d \rho\left(A_{0}\right) x_{9}=0, d \rho^{*}\left(A_{0}\right) y_{9}=y_{9}$. Since $\delta \chi\left(A_{0}\right)=3 \operatorname{tr} A_{0}=-3, \operatorname{tr}_{V_{x_{9}}^{*}} A_{0}=9 \varepsilon=-9, \operatorname{dim} V_{x_{9}}^{*}=9$, we have $\operatorname{ord}_{\Lambda_{9}} f^{s}=$ $-3 s-\frac{9}{2}$. Let $A_{\beta}$ be an element of $g_{x_{9}}$ with $\varepsilon=((\beta+2) / 3), X=\left(\begin{array}{lll}\eta & & \\ & \eta & \\ & & -2 \eta\end{array}\right)$ with $\eta=((1-\beta) / 3)$, all remaining parts zero in (10.6). Then we have $d \rho\left(A_{0}\right) x_{9}=0, d \rho^{*}\left(A_{0}\right) y_{9}^{\prime}=y_{9}^{\prime}$ and $\operatorname{tr}_{\tilde{V}} A_{0}=\beta$ where $\tilde{V}=V_{x_{9}}^{*} \bmod d \rho_{x_{9}}\left(\mathrm{~g}_{x_{9}}\right) y_{9}^{\prime}=$ $C u_{3} \wedge u_{6} \wedge u_{7}$. This implies that $\Lambda_{4}$ and $\Lambda_{9}$ intersect regularly. By Corollary $1-2$, we have $b_{\Lambda_{9}}(s) / b_{\Lambda_{4}}(s)=(s+3)$.

(6) The isotropy subalgebra $\mathfrak{g}_{x_{10}}$ at $x_{10}$ is given as follows.

$$
\begin{aligned}
\mathfrak{g}_{x_{10}}= & \left\{A=\left(\begin{array}{c|c|c}
\varepsilon I_{3}+X & B & C \\
\hline 0 & -2 \varepsilon I_{3}+X & F \\
\hline 0 & 0 & \eta
\end{array}\right) ; X \in \mathfrak{g l}(3), \operatorname{tr} B=0, C, D \in C^{3}\right\} \\
& \cong(g r(1) \oplus \operatorname{gr}(3)) \oplus \mathfrak{u}(14) .
\end{aligned}
$$

Put $\omega_{1}=u_{5} \wedge u_{6} \wedge u_{7}, \omega_{2}=u_{4} \wedge u_{6} \wedge u_{7}, \omega_{3}=u_{4} \wedge u_{5} \wedge u_{7}, \omega_{4}=u_{1} \wedge u_{4}$ $\wedge u_{7}, \omega_{5}=u_{2} \wedge u_{5} \wedge u_{7}, \omega_{6}=u_{3} \wedge u_{6} \wedge u_{7}, \omega_{7}=\left(u_{1} \wedge u_{5}+u_{2} \wedge u_{4}\right) \wedge u_{7}$, $\omega_{8}=\left(u_{2} \wedge u_{6}+u_{3} \wedge u_{5}\right) \wedge u_{7}, \omega_{9}=\left(u_{1} \wedge u_{6}+u_{3} \wedge u_{4}\right) \wedge u_{7}, \omega_{10}=u_{4} \wedge u_{5} \wedge$ $u_{6}$. Then the conormal vector space $V_{x_{10}}^{*}$ is spanned by $\omega_{1}, \cdots, \omega_{10}$, and the action $d \rho_{x_{10}}$ of $\mathfrak{g}_{x_{10}}$ on $V_{x_{10}}^{*}$ is given as follows.

$$
d \rho_{x_{10}}(A)\left(\omega_{1}, \cdots, \omega_{10}\right)=\left(\omega_{1}, \cdots, \omega_{10}\right)\left(\begin{array}{c|c}
\frac{(4 \varepsilon-\eta) I_{3}+X}{0} \\
\hline 0
\end{array} \mid \begin{array}{c|c}
\frac{B^{\prime}}{(\varepsilon-\eta) I_{6}+d \rho_{1}^{*}(X)_{i}^{*}} & 0 \\
\hline 0 & 6 \varepsilon
\end{array}\right)
$$

where $\rho_{1}=2 \Lambda_{1}$ for $S L(3)$. 
Then $y_{10}=\omega_{4}+\omega_{8}+\omega_{10}$ is a generic point. There exist two one-codimensional orbits. As a representative point, we may take $y_{10}^{\prime}=\omega_{8}+\omega_{10}$ and $y_{10}^{\prime \prime}=\omega_{4}+\omega_{5}+\omega_{6}$ respectively. This implies that $\operatorname{dim} \Lambda_{7} \cap \Lambda_{10}=\operatorname{dim} \Lambda_{9}$ $\cap \Lambda_{10}=\operatorname{dim} V-1$. Since $\Lambda_{10}=\Lambda_{7}^{*}, \Lambda_{10}$ is a good holonomic variety. Let $A_{0}$ be an element of $g_{x_{10}}$ with $\varepsilon=\frac{1}{6}, \eta=-\frac{5}{6}$, all remaining parts zero in (10.7). Then $d \rho\left(A_{0}\right) x_{10}=0$ and $d \rho^{*}\left(A_{0}\right) y_{10}=y_{10}$. Since $\delta \chi\left(A_{0}\right)=-9 \varepsilon+3 \eta$ $=-4, \operatorname{tr}_{r_{x_{10}}^{*}} A_{0}=24 \varepsilon-9 \eta=\frac{23}{2}$, and $\operatorname{dim} V_{x_{10}}^{*}=10$, we have $\operatorname{ord}_{\Lambda_{10}} f^{s}=$ - $4 s-\frac{13}{2}$ by Proposition 1-3.

Since $d \rho^{*}\left(A_{0}\right) y_{10}^{\prime}=y_{10}^{\prime}$ and $\operatorname{tr}_{\tilde{V}} A_{0}=1$ where $\tilde{V}=V_{x_{10}}^{*} \bmod d \rho_{x_{10}}\left(\mathfrak{g}_{x_{10}}\right) y_{10}^{\prime}$ $=C \omega_{4}, \Lambda_{7}$ and $\Lambda_{10}$ intersect regularly by Proposition 1-4. Let $A_{\beta}$ be an element of $g_{x_{10}}$ with $\varepsilon=\frac{\beta}{6}, \eta=\frac{\beta}{6}-1$, all remaining parts zero in (10.7). Then we have $d \rho\left(A_{\beta}\right) x_{10}=0, d \rho^{*}\left(A_{\beta}\right) y_{10}^{\prime \prime}=y_{10}^{\prime \prime}$, and $\operatorname{tr}_{\tilde{v}} A_{\beta}=\beta$ where $\tilde{V}=$ $V_{x_{10}}^{*} \bmod d \rho_{\rho_{10}}\left(g_{x_{10}}\right) y_{10}^{\prime \prime}=C \omega_{10}$. This implies that $\Lambda_{9}$ and $\Lambda_{10}$ intersect regularly by Proposition 1-4. By Corollary 1-2, we have $b_{1_{10}}(s) / b_{\lambda_{7}}(s)=(s+3)$ and $b_{\Lambda_{10}}(s) / b_{\Lambda_{9}}(s)=\left(s+\frac{5}{2}\right)$.

(7) The isotropy subalgebra $\mathfrak{g}_{x_{14}}$ at $x_{14}$ is given as follows.

$$
\mathfrak{g}_{x_{14}}=\left\{\left(\frac{-2 \varepsilon}{0} \mid \frac{Y}{\varepsilon I_{6}+X}\right) ; X \in \mathfrak{Z}_{\mathfrak{p}}(3),{ }^{t} Y \in C^{6}\right\} \cong(\mathfrak{g}(1) \oplus \mathfrak{z} \mathfrak{p}(3)) \oplus V(6) .
$$

Put $\omega_{1}=u_{2} \wedge u_{3} \wedge u_{4}, \omega_{2}=u_{5} \wedge u_{6} \wedge u_{7}, \omega_{3}=u_{3} \wedge u_{4} \wedge u_{5}, \omega_{4}=u_{2} \wedge u_{6} \wedge$ $u_{7}, \omega_{5}=u_{2} \wedge u_{4} \wedge u_{6}, \omega_{6}=u_{3} \wedge u_{5} \wedge u_{7}, \omega_{7}=u_{2} \wedge u_{3} \wedge u_{7}, \omega_{8}=u_{4} \wedge u_{5} \wedge$ $u_{6}, \omega_{9}=u_{2} \wedge u_{3} \wedge u_{6}-u_{2} \wedge u_{4} \wedge u_{7}, \omega_{10}=u_{3} \wedge u_{5} \wedge u_{6}-u_{4} \wedge u_{5} \wedge u_{7}, \omega_{11}$ $=u_{2} \wedge u_{3} \wedge u_{5}+u_{3} \wedge u_{4} \wedge u_{7}, \omega_{12}=u_{2} \wedge u_{5} \wedge u_{6}+u_{4} \wedge u_{6} \wedge u_{7}, \omega_{13}=u_{2}$ $\wedge u_{4} \wedge u_{5}-u_{3} \wedge u_{4} \wedge u_{6}, \omega_{14}=u_{2} \wedge u_{5} \wedge u_{7}-u_{3} \wedge u_{6} \wedge u_{7}$. The conormal vector space $V_{x_{14}}^{*}$ is spanned by these $\omega_{1}, \cdots, \omega_{14}$. By seeing the weights, we have $\left(G_{x_{14}}, \rho_{x_{14}}, V_{x_{14}}^{*}\right) \cong\left(G L(1) \times S p(3), \square \otimes \Lambda_{3}, V(1) \otimes V(14)\right)$. Since this is an irreducible regular P.V., $\Lambda_{14}$ is a good holonomic variety by Corollary 1-8. As we have seen in $\S 9, y_{14}=\omega_{1}+\omega_{2}$ is a generic point. Let $A_{0}$ be an element of $g_{x_{14}}$ with $\varepsilon=-\frac{1}{3}, X=Y=0$ in (10.9). Then $d \rho\left(A_{0}\right) x_{14}=0$ and $d \rho^{*}\left(A_{0}\right) y_{14}=y_{14}$. Since $\delta \chi\left(A_{0}\right)=3 \operatorname{tr} A_{0}=-4, \operatorname{tr}_{V x_{14}}^{*} A_{0}=14 \times 3 \varepsilon=$ -14 and $\operatorname{dim} V_{x_{14}}^{*}=14$, we have $\operatorname{ord}_{\Lambda_{14}} f^{s}=-4 s-\frac{14}{2}$. Since $\Lambda_{14}=\Lambda_{9}^{*}, \Lambda_{7}=$ $\Lambda_{10}^{*}$, and $\Lambda_{9}$ and $\Lambda_{10}$ intersect regularly with codimension one, so do $\Lambda_{14}$ and $\Lambda_{7}$. By Corollary 1-2, we have $b_{\Lambda_{14}}(s) / b_{\Lambda_{7}}(s)=\left(s+\frac{7}{2}\right)$.

(8) The isotropy subalgebra $\mathfrak{g}_{x_{15}}$ at $x_{15}$ is given as follows.

$$
\left.\mathfrak{g}_{x_{15}}=\left\{\begin{array}{c|c|c}
-2 \varepsilon & W & U \\
\hline 0 & \varepsilon I_{4}+X & Z \\
\hline 0 & 0 & \eta I_{2}+Y
\end{array}\right) ; \begin{array}{l}
X \in \mathfrak{g} p(2), Y \in \mathfrak{s l}(2), Z \in M(4,2) \\
{ }^{t} W \in C^{2},{ }^{t} U \in C^{4}, \varepsilon, \eta \in C
\end{array}\right\}
$$




$$
\cong(\mathfrak{g} \mathfrak{l}(1) \oplus \mathfrak{g} \mathfrak{p}(2) \oplus \mathfrak{g} \mathfrak{l}(2)) \oplus \mathfrak{u}(14)
$$

Put $\omega_{i}=u_{i+1} \wedge u_{6} \wedge u_{7}(1 \leq i \leq 4), \omega_{5}=u_{1} \wedge u_{6} \wedge u_{7}, \omega_{6+j}=\left(u_{2} \wedge u_{4}-u_{3}\right.$ $\left.\wedge u_{5}\right) \wedge u_{6+j}, \omega_{8+j}=u_{2} \wedge u_{3} \wedge u_{6+j}, \omega_{10+j}=u_{2} \wedge u_{5} \wedge u_{6+j}, \omega_{12+j}=u_{3} \wedge u_{4} \wedge$ $u_{6+j}, \omega_{14+j}=u_{4} \wedge u_{5} \wedge u_{6+j}(j=0,1)$. Then the conormal vector space $V_{x_{15}}^{*}$ is spanned by $\omega_{1}, \cdots, \omega_{15}$. The action $d \rho_{x_{15}}$ of $g_{x_{15}}$ on $V_{x_{15}}^{*}$ is as follows.

$$
\begin{aligned}
& d \rho_{x_{15}}(A)\left(\omega_{1}, \cdots, \omega_{15}\right)=\left(\omega_{1}, \cdots, \omega_{15}\right) \\
& \quad \times\left(\begin{array}{c|c|c}
-(\varepsilon+2 \eta) I_{4}+d \rho_{1}(X) & * & * \\
\hline 0 & 2 \varepsilon-2 \eta & * \\
\hline 0 & 0 & -(2 \varepsilon+\eta) I_{10}+d \rho_{2}^{*}(X \oplus Y)
\end{array}\right)
\end{aligned}
$$

where $\rho_{1}=\Lambda_{1}$ for $S p(2)$ and $\rho_{2}=\Lambda_{2} \otimes \Lambda_{1}$ for $S p(2) \times S L(2)$. Since $\Lambda_{15}=$ $\Lambda_{4}^{*}$ and $\Lambda_{4}$ is a good holonomic variety, $\Lambda_{15}$ is also a good holonomic variety. $y_{15}=\omega_{5}+\omega_{11}+\omega_{12}$ is a generic point. Let $A_{0}$ be an element of $\mathfrak{g}_{x_{15}}$ with $\varepsilon=-\frac{1}{6}, \eta=-\frac{2}{3}$, all remaining parts zero in (10.10). Then $d \rho\left(A_{0}\right) x_{15}=0$ and $d \rho^{*}\left(A_{0}\right) y_{15}=y_{15}$. Since $\delta \chi\left(A_{0}\right)=3 \operatorname{tr} A_{0}=6(\varepsilon+\eta)=-5$, $\operatorname{tr}_{V_{x_{15}}^{*}} A_{0}=-22 \varepsilon-20 \eta=\frac{102}{6}$, and $\operatorname{dim} V_{x_{15}}^{*}=15$, we have $\operatorname{ord}_{\Lambda_{15}} f^{s}=-5 s-$ $\frac{19}{2}$. Since $\Lambda_{15}=\Lambda_{4}^{*}, \Lambda_{14}=\Lambda_{9}^{*}, \Lambda_{10}=\Lambda_{7}^{*}$, we have $\operatorname{dim} \Lambda_{15} \cap \Lambda_{14}=\operatorname{dim} \Lambda_{15} \cap$ $\Lambda_{10}=\operatorname{dim} V-1$ and they intersect regularly. By Corollary 1-2, we have $b_{\Lambda_{15}}(s) / b_{\Lambda_{14}}(s)=(s+3)$ and $b_{\Lambda_{15}}(s) / b_{\Lambda_{10}}(s)=\left(s+\frac{7}{2}\right)$.

(9) The isotropy subalgebra $\mathfrak{g}_{x_{22}}$ at $x_{22}$ is given as follows.

$$
\begin{aligned}
\mathfrak{g}_{x_{22}}= & \left\{\tilde{X}=\left(\frac{X}{Z} \mid \frac{Z}{\varepsilon I_{4}+Y}\right) ; X \in \mathfrak{g l}(3), Y \in \mathfrak{g l}(4), Z \in M(3,4)\right\} \\
& \cong(\mathfrak{g l}(3) \oplus \mathfrak{g}((4)) \oplus V(12) .
\end{aligned}
$$

The conormal vector space $V_{x_{22}}^{*}$ is spanned by $u_{i} \wedge u_{j} \wedge u_{k}(4 \leq i<j<$ $k \leq 7)$ and $u_{i} \wedge u_{j} \wedge u_{k}(1 \leq i \leq 3,4 \leq j<k \leq 7)$. The action $d \rho_{x_{22}}$ of $g_{x_{22}}$ is given by

$$
\begin{aligned}
& d \rho(\tilde{X})\left(u_{5} \wedge u_{6} \wedge u_{7}, \cdots\right) \\
& =\left(u_{5} \wedge u_{6} \wedge u_{7}, \cdots\right)\left(\begin{array}{c|c}
Y-3 \varepsilon I_{4} & * \\
\hline 0 & -2 \varepsilon I_{18}+d \rho_{1}^{*}(X \oplus Y)
\end{array}\right)
\end{aligned}
$$

where $\rho_{1}=\Lambda_{1} \otimes \Lambda_{2}$ for $S L(3) \times S L(4)$. For example, $y_{22}=u_{1} \wedge\left(u_{1} \wedge u_{5}+\right.$ $\left.u_{6} \wedge u_{7}\right)+u_{2} \wedge u_{4} \wedge u_{6}+u_{3} \wedge u_{5} \wedge u_{7}$ is a generic point. Since $A_{22}=\Lambda_{1}^{*}$, $A_{22}$ is a good holonomic variety. Let $A_{0}$ be an element of $\mathrm{g}_{x_{22}}$ with $\varepsilon=-\frac{1}{2}$, $X=Y=Z=0$ in (10.12). Then $d \rho\left(A_{0}\right) x_{22}=0$ and $d \rho^{*}\left(A_{0}\right) y_{22}=y_{22}$. Since $\delta \chi\left(A_{0}\right)=12 \varepsilon=-6, \operatorname{tr}_{V \cdot x_{22}}^{*} A_{0}=-48 \varepsilon=24$ and $\operatorname{dim} V_{x_{22}}^{*}=22$, we have $\operatorname{ord}_{A_{22}}$ $f^{s}=-6 s-\frac{26}{2}(=-6 s-13)$. Since $A_{22}=\Lambda_{1}^{*}$ and $\Lambda_{15}=\Lambda_{4}^{*}$, we have 
$\operatorname{dim} \Lambda_{22} \cap \Lambda_{15}=\operatorname{dim} V-1$ and they intersect regularly.

By Corollary 1-2, we have $b_{\Lambda_{22}}(s) / b_{\Lambda_{15}}(s)=(s+4)$.

(10) The isotropy subalgebra $g_{x_{35}}$ at $x_{35}=0$ is $g$ itself and we have $\left(G_{x_{35}}, \rho_{x_{35}}, V_{x_{35}}^{*}\right)=\left(G, \rho^{*}, V^{*}\right) \cong\left(G L(7), \Lambda_{3}, V(35)\right)$. Then $y_{35}=x_{0}=u_{2} \wedge u_{3} \wedge$ $\wedge u_{4}+u_{5} \wedge u_{6} \wedge u_{7}+u_{1} \wedge\left(u_{2} \wedge u_{5}+u_{3} \wedge u_{6}+u_{4} \wedge u_{7}\right)$ is its generic point. Put $A_{0}=-\frac{1}{3} I_{7}$. Then $d \rho\left(A_{0}\right) x_{35}=0$ and $d \rho^{*}\left(A_{0}\right) y_{35}=y_{35}$. Since $\delta \chi\left(A_{0}\right)=$ $3 \operatorname{tr} A_{0}=-7, \operatorname{tr}_{V_{x_{35}}^{*}} A_{0}=-35$ and $\operatorname{dim} V_{x_{35}}^{*}=35$, we have ord ${ }_{\Lambda_{35}} f^{s}=-7 s$ - $\frac{35}{2}$. Since $\Lambda_{22}=\Lambda_{1}^{*}$ and $\Lambda_{35}=\Lambda_{0}^{*}$, they intersect regularly with codimension one. By Corollary $1-2$, we have $b_{A_{35}}(s) / b_{\Lambda_{22}}(s)=(s+5)$. Since $b_{\Lambda_{0}}(s)=1$ and $b_{\Lambda_{35}}(s)=b(s)$, we obtain the $b$-function $b(s)=(s+1)(s+2)$ $\left(s+\frac{5}{2}\right)\left(s+\frac{7}{2}\right)(s+3)(s+4)(s+5)$, and the holonomy diagram (Figure 101). We denote $\Lambda_{m}$ by $m$.

Note that the colocalization at $x_{1}, x_{4}, x_{7}, x_{9}, x_{14}, x_{22}$ and $x_{35}$ has the unique one-codimensional orbit respectively, and the colocalization at $x_{10}$ and $x_{15}$ has the two one-codimensional orbits respectively. Therefore we have obtained all one-codimensional intersections among the conormal bundles.

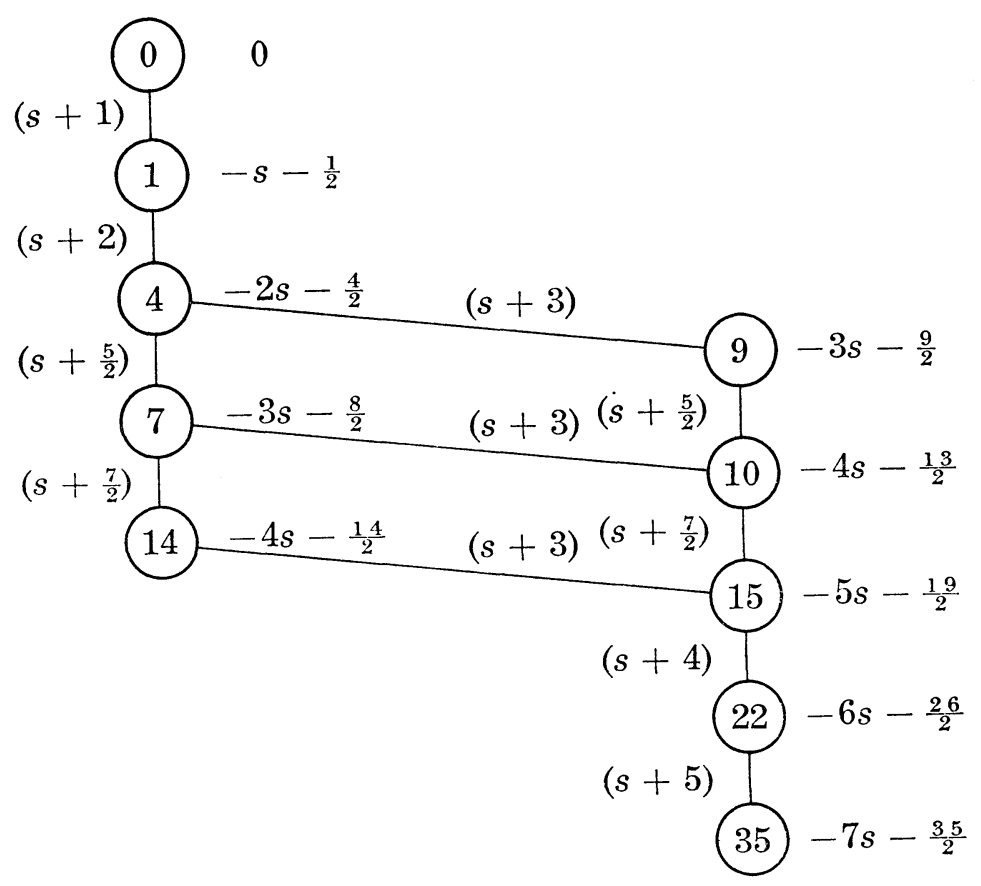

Figure 10-1. Holonomy diagram of $\left(G L(7), \Lambda_{3}, V(35)\right)$. 


\section{$\S 11 . \quad\left(S L(5) \times G L(3), \Lambda_{2} \otimes \Lambda_{1}, V(10) \otimes V(3)\right)$}

Let $V(10)$ be a vector space spanned by 2 -forms $u_{i} \wedge u_{j}(1 \leq i<j \leq$ 5). Then the representation space is identified with $V=V(10) \oplus V(10) \oplus$ $V(10)$ (See [1]). Let $\Lambda$ be the conormal bundle of an orbit $S$ in $V$ and $\Lambda^{*}$ that of an orbit $S^{*}$ in $V^{*}$. When $\Lambda=\Lambda^{*}$, we say that $S$ and $S^{*}$ are the dual orbits of each other. We denote by $S_{i, j}^{(k)}$ the $i$-codimensional orbit whose dual orbit is $j$-codimensional, where $k$ denotes the dimension of the central torus of the isotropy subgroup of this orbit. When there is no confusion, denote this by $S_{i}$ or $S_{i j}$. We denote by $\Lambda_{i, j}^{(k)}$ (resp. $\Lambda_{i, j}, \Lambda_{i}$ ) the conormal bundle of $S_{i, j}^{(k)}$ (resp. $S_{i, j}, S_{i}$ ). We identify $V$ and its dual $V^{*}$ by taking $\left(u_{i} \wedge u_{i^{\prime}}, u_{j} \wedge u_{j^{\prime}}, u_{k} \wedge u_{k^{\prime}}\right)\left(i<i^{\prime}, j<j^{\prime}, k<k^{\prime}\right)$ as a dual basis.

Proposition 11-1. This space has following twenty five orbits $S_{i, j}^{(k)}$.

(1) $S_{0,30}^{(0)}:\left(u_{1} \wedge u_{2}+u_{3} \wedge u_{4}, u_{2} \wedge u_{3}+u_{4} \wedge u_{5}, u_{1} \wedge u_{3}+u_{2} \wedge u_{5}\right)\left(=x_{0}\right)$

(2) $S_{1,21}^{(2)}:\left(u_{1} \wedge u_{2}, u_{1} \wedge u_{5}+u_{3} \wedge u_{4}, u_{2} \wedge u_{3}+u_{4} \wedge u_{5}\right)\left(=x_{1}\right)$

(3) $S_{2,16}^{(2)}:\left(u_{1} \wedge u_{2}, u_{2} \wedge u_{3}+u_{3} \wedge u_{4}, u_{1} \wedge u_{3}+u_{4} \wedge u_{5}\right)\left(=x_{2}\right)$

(4) $S_{3,15}^{(3)}:\left(u_{1} \wedge u_{2}, u_{3} \wedge u_{4}, u_{1} \wedge u_{5}+u_{4} \wedge u_{5}\right)\left(=x_{3}\right)$

(5) $S_{3,13}^{(2)}:\left(u_{1} \wedge u_{2}, u_{1} \wedge u_{3}+u_{2} \wedge u_{4}, u_{2} \wedge u_{3}+u_{4} \wedge u_{5}\right)\left(=x_{3}^{\prime}\right)$

(6) $S_{4,11}^{(3)}:\left(u_{1} \wedge u_{2}, u_{1} \wedge u_{3}+u_{2} \wedge u_{4}, u_{4} \wedge u_{5}\right)\left(=x_{4}\right)$

(7) $S_{5,8}^{(2)}:\left(u_{1} \wedge u_{2}, u_{1} \wedge u_{3}+u_{2} \wedge u_{4}, u_{1} \wedge u_{5}+u_{3} \wedge u_{4}\right)\left(=x_{5}\right)$

(8) $S_{6,12}^{(1)}:\left(u_{1} \wedge u_{2}, u_{1} \wedge u_{3}, u_{2} \wedge u_{3}+u_{4} \wedge u_{5}\right)\left(=x_{6}\right)$

(9) $S_{7,9}^{(2)}:\left(u_{1} \wedge u_{2}, u_{1} \wedge u_{3}, u_{4} \wedge u_{5}\right)\left(=x_{7}\right)$

(10) $S_{7,7}^{(1)}:\left(u_{1} \wedge u_{2}, u_{1} \wedge u_{3}+u_{2} \wedge u_{4}, u_{2} \wedge u_{3}+u_{1} \wedge u_{5}\right)\left(=x_{7}^{\prime}\right)$

(11) $S_{7,7}^{(2)}:\left(u_{1} \wedge u_{2}, u_{1} \wedge u_{3}, u_{2} \wedge u_{4}+u_{3} \wedge u_{5}\right)\left(=x_{7}^{\prime \prime}\right)$

(12) $S_{8,18}^{(2)}:\left(u_{1} \wedge u_{2}+u_{3} \wedge u_{4}, u_{2} \wedge u_{3}+u_{4} \wedge u_{5}, 0\right)\left(=x_{8}\right)$

(13) $S_{8,14}^{(1)}:\left(u_{1} \wedge u_{2}, u_{3} \wedge u_{4}, u_{1} \wedge u_{3}+u_{2} \wedge u_{4}\right)\left(=x_{8}^{\prime}\right)$

(14) $S_{8,5}^{(3)}:\left(u_{1} \wedge u_{2}, u_{1} \wedge u_{3}, u_{1} \wedge u_{5}+u_{2} \wedge u_{4}\right)\left(=x_{8}^{\prime \prime}\right)$

(15) $S_{9,7}^{(4)}:\left(u_{1} \wedge u_{2}, u_{1} \wedge u_{3}, u_{2} \wedge u_{4}\right)\left(=x_{9}\right)$

(16) $S_{10,10}^{(3)}:\left(u_{1} \wedge u_{2}, u_{3} \wedge u_{4}+u_{1} \wedge u_{5}, 0\right)\left(=x_{10}\right)$

(17) $S_{11,4}^{(2)}:\left(u_{1} \wedge u_{2}, u_{1} \wedge u_{3}, u_{1} \wedge u_{4}+u_{2} \wedge u_{3}\right)\left(=x_{11}\right)$

(18) $S_{12,6}^{(3)}:\left(u_{1} \wedge u_{2}, u_{3} \wedge u_{4}, 0\right)\left(=x_{12}\right)$

(19) $S_{13,3}^{(3)}:\left(u_{1} \wedge u_{2}, u_{1} \wedge u_{3}+u_{2} \wedge u_{4}, 0\right)\left(=x_{13}\right)$

(20) $S_{14,8}^{(2)}:\left(u_{1} \wedge u_{2}, u_{1} \wedge u_{3}, u_{1} \wedge u_{4}\right)\left(=x_{14}\right)$

(21) $S_{15,3}^{(1)}:\left(u_{1} \wedge u_{2}, u_{1} \wedge u_{3}, u_{2} \wedge u_{3}\right)\left(=x_{15}\right)$

(22) $S_{16,2}^{(3)}:\left(u_{1} \wedge u_{2}, u_{1} \wedge u_{3}, 0\right)\left(=x_{16}\right)$

(23) $S_{18,8}^{(2)}:\left(u_{1} \wedge u_{2}+u_{3} \wedge u_{4}, 0,0\right)\left(=x_{18}\right)$

(24) $S_{21,1}^{(2)}:\left(u_{1} \wedge u_{2}, 0,0\right)\left(=x_{21}\right)$ 
(25) $S_{30,0}^{(1)}:(0,0,0)\left(=x_{30}\right)$.

Proof. It is easy to check that the non-regular P.V. $(S L(5) \times G L(2)$, $\left.\Lambda_{2} \otimes \Lambda_{1}, V(10) \otimes V(2)\right)$ has eight orbits which are represented by the following points; [1] $(0,0)$, [2] $\left(u_{1} \wedge u_{2}, 0\right)$, [3] $\left(u_{1} \wedge u_{2}+u_{3} \wedge u_{4}, 0\right)$, [4] $\left(u_{1} \wedge u_{2}, u_{1} \wedge\right.$ $\left.u_{3}\right)$, [5] $\left(u_{1} \wedge u_{2}, u_{1} \wedge u_{3}+u_{2} \wedge u_{4}\right)$, [6] $\left(u_{1} \wedge u_{2}, u_{3} \wedge u_{4}\right)$, [7] $\left(u_{1} \wedge u_{2}, u_{3} \wedge u_{4}\right.$ $\left.+u_{1} \wedge u_{5}\right)$, [8] $\left(u_{1} \wedge u_{2}+u_{3} \wedge u_{4}, u_{2} \wedge u_{3}+u_{4} \wedge u_{5}\right)$. Therefore, for a point $x=\left(x_{1}, x_{2}, x_{3}\right)$ of $V$, we may assume that $\left(x_{1}, x_{2}\right)$ is one of these points. In the first three cases, repeating the same argument, we obtain (12), (16), (18), (19), (22), (23), (24) and (25). For $\lambda \in C$, we define $S_{i j}(\lambda)$ by $S_{i j}(\lambda) u_{k}=$ $u_{k}$ for $k \neq i$ and $S_{i j}(\lambda) u_{i}=u_{i}+\lambda u_{j}$. Then $S_{i j}(\lambda)$ is an element of $\rho(G)$. Put $x_{3}=\sum_{i<j} a_{i j} u_{i} \wedge u_{j}$. First we consider the case [4], i.e., $\left(x_{1}, x_{2}\right)=\left(u_{1} \wedge\right.$ $u_{2}, u_{1} \wedge u_{3}$ ). Assume that $a_{45} \neq 0$. Then we may assume that $x_{3}=a_{23} u_{2} \wedge$ $u_{3}+u_{4} \wedge u_{5}$. In fact, we have $a_{35}=0$ by $S_{43}\left(-a_{35} / a_{45}\right)$ and so on. If $a_{23}$ $=0$, then we have (9). If $a_{23} \neq 0$, then we have (8). Next assume that $a_{45}=0$. If one of $a_{i j}(i=2,3 ; j=4,5)$ is not zero, we may assume that $x_{3}=u_{2} \wedge u_{4}+a_{15} u_{1} \wedge u_{5}+a_{35} u_{3} \wedge u_{5}$. If $a_{35} \neq 0$ (resp. $a_{35}=0$ and $a_{15} \neq 0$, $\left.a_{35}=a_{15}=0\right)$, then we have (11) (resp. (14), (15)). If any $a_{i j}=0(i=2,3$; $j=4,5)$, then we may assume that $x_{3}=a_{14} u_{1} \wedge u_{4}+a_{23} u_{2} \wedge u_{3}$. If $a_{14} \neq 0$ and $a_{23} \neq 0$ (resp. $a_{14} \neq 0$ and $a_{23}=0, a_{14}=0$ and $a_{23} \neq 0, a_{14}=a_{23}=0$ ), then we have (17) (resp. (20), (21), (22)). Next we consider the case [5], i.e., $\left(x_{1}, x_{2}\right)=\left(u_{1} \wedge u_{2}, u_{1} \wedge u_{3}+u_{2} \wedge u_{4}\right)$. If $a_{35} \neq 0$ or $a_{45} \neq 0$, we may assume that $x_{3}=a_{23} u_{2} \wedge u_{3}+u_{4} \wedge u_{5}$ and hence we have (5) (resp. (6)) for $a_{23} \neq 0$ (resp. $a_{23}=0$ ). If $a_{35}=a_{45}=0$ and one of $a_{k 5}(k=1,2)$ is not zero, then we may assume that $x_{3}=a_{23} u_{2} \wedge u_{3}+a_{34} u_{3} \wedge u_{4}+u_{1} \wedge u_{5}$ and hence we have (7) (resp. (10), (14)) for $a_{34} \neq 0$ (resp. $a_{34}=0$ and $a_{23} \neq 0$, $a_{34}=a_{23}=0$ ). If $a_{k 5}=0$ for $1 \leq k \leq 4$, we may assume that $x_{3}=a_{13} u_{1} \wedge$ $u_{3}+a_{14} u_{1} \wedge u_{4}+a_{23} u_{2} \wedge u_{3}+a_{34} u_{3} \wedge u_{4}$. Then we have (13) (resp. we have (19); it is reduced to the case [4]) for $a_{34} \neq 0$ (resp. $x_{3}=0 ; a_{34}=0$ and $x_{3}$ $\neq 0)$. Now we consider the case [6], i.e., $\left(x_{1}, x_{2}\right)=\left(u_{1} \wedge u_{2}, u_{3} \wedge u_{4}\right)$. (i) If $a_{35} \neq 0$ or $a_{45} \neq 0$, we may assume that $x_{3}=a_{12} u_{1} \wedge u_{3}+a_{15} u_{1} \wedge u_{5}+$ $a_{25} u_{2} \wedge u_{5}+u_{4} u_{5}$. Moreover if $a_{25} \neq 0$, then we have (3) (resp. (4)) for $a_{13}$ $\neq 0$ (resp. $a_{13}=0$ ). If $a_{25}=0$, then we have (4) (resp. it is reduced to the case [4] or [5]) for $a_{15} \neq 0$ (resp. $a_{15}=0$ ). (ii) If $a_{35}=a_{45}=0$, it is reduced to the previous cases. Next we shall consider the case [7], i.e., $\left(x_{1}, x_{2}\right)=$ $\left(u_{1} \wedge u_{2}, u_{3} \wedge u_{4}+u_{1} \wedge u_{5}\right)$. (i) If $a_{35} \neq 0$ or $a_{45} \neq 0$, then we may assume that $a_{45}=1$ and $a_{35}=a_{24}=a_{14}=a_{12}=0$. By $S_{53}(\lambda), S_{41}(\mu), S_{21}(\nu)$ and $G L(3)$, we have $x_{3}=\left(a_{13}+\left(a_{15}-a_{34}\right) \lambda+\left(a_{23}+a_{25}\right) \nu+\lambda^{2}\right) u_{1} \wedge u_{3}+\left(a_{23}+\lambda a_{25}\right) u_{2} \wedge u_{3}$ 
$+a_{25} u_{2} \wedge u_{5}+\left(a_{15}+\nu a_{25}+\mu+\lambda\right) u_{1} \wedge u_{5}+\left(a_{34}+\mu-\lambda\right) u_{3} \wedge u_{4}+u_{4} \wedge u_{5}$. If $a_{25} \neq 0$, we may take $\lambda, \mu$, $\nu$ so that $a_{13}+\left(a_{15}-a_{34}\right) \lambda+\nu\left(a_{23}+\lambda a_{25}\right)+\lambda^{2}$ $=a_{15}+\nu a_{25}+\mu+\lambda=a_{34}+\mu-\lambda=0$ and hence we have $x_{3}=\alpha u_{2} \wedge u_{3}$ $+u_{2} \wedge u_{5}+u_{4} \wedge u_{5}$. If $\alpha \neq 0$ (resp. $\alpha=0$ ), then we have (2) (resp. (3)) by $S_{35}(-1 / 2 \alpha), S_{42}(-1 / 2), S_{12}(1 / 4 \alpha),\left\{u_{3} \mapsto(1 / \sqrt{\alpha}) u_{3}, u_{4} \mapsto \sqrt{\alpha} u_{4}, u_{j} \mapsto u_{j}(j \neq\right.$ $3,4)\}$ and $\left(\begin{array}{ccc}1 & 0 & 0 \\ 0 & 1 & -1 / 2 \alpha \\ 0 & 0 & 1 / \sqrt{\alpha}\end{array}\right)$ (resp. by $\left(\begin{array}{lll}1 & 0 & 0 \\ 0 & 0 & 1 \\ 0 & 1 & 1\end{array}\right)$ and $\left\{u_{5} \mapsto u_{3}, u_{3} \mapsto u_{5}, u_{4} \mapsto\right.$ $\left.-u_{4}, u_{j} \mapsto u_{j}(j=1,2)\right)$. If $a_{25}=0$, taking $\lambda$ and $\mu$ satisfying $a_{15}+\mu+\lambda$ $=a_{34}+\mu-\lambda=0$, we have $x_{3}=a_{13}^{\prime} u_{1} \wedge u_{3}+a_{23}^{\prime} u_{2} \wedge u_{3}+u_{4} \wedge u_{5}$. If $a_{23}^{\prime}$ $\neq 0$ (resp. $a_{23}^{\prime}=a_{13}^{\prime}=0$ ), then we have (2) (resp. (6)). If $a_{23}^{\prime}=0$ and $a_{13}^{\prime} \neq$ 0 , then we have (4) by $S_{41}(\lambda),\left(\begin{array}{ccc}1 & & -1 / \gamma \\ & 0 & 1\end{array}\right), S_{35}\left(-\frac{1}{\gamma}\right), S_{53}\left(\frac{\gamma}{2}\right),\left(\begin{array}{ccc}1 & & 1 \\ \gamma / 2 & 1\end{array}\right)$

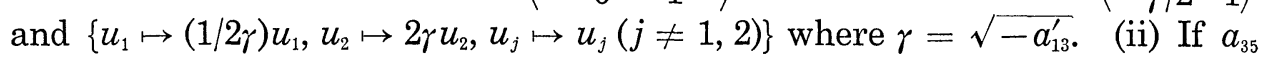
$=a_{45}=0$, it is reduced to the previous cases. Finally we shall consider the case [8], i.e., $\left(x_{1}, x_{2}\right)=\left(u_{1} \wedge u_{2}+u_{3} \wedge u_{4}, u_{2} \wedge u_{3}+u_{4} \wedge u_{5}\right)$. The isotropy subalgebra $\mathfrak{h}$ of $\mathfrak{g l}(5) \oplus \mathfrak{g}(2)$ at this point $\left(x_{1}, x_{2}\right)$ is given by

$$
\mathfrak{h}=\left\{\left(\begin{array}{ccccc}
a_{1} & & \gamma_{1} & & \\
\alpha_{1} & a_{2} & \beta_{1} & \gamma_{2} & \beta_{2} \\
-2 \gamma_{2} & & a_{3} & & 2 \gamma_{1} \\
\beta_{1} & -\gamma_{2} & \beta_{2} & a_{4} & \alpha_{2} \\
& & -\gamma_{2} & & a_{5}
\end{array}\right) \oplus\left(\begin{array}{ccc}
-\left(a_{1}+a_{2}\right) & -\gamma_{2} \\
\gamma_{1} & -\left(a_{4}+a_{5}\right)
\end{array}\right)\right\}
$$

with $a_{1}+a_{2}=a_{3}+a_{4}, a_{2}+a_{3}=a_{4}+a_{5}$ and $\sum_{i=1}^{5} a_{i}=0$.

Taking one-parameter subgroups from $\mathfrak{h}$, we obtain the following actions which fix $\left(x_{1}, x_{2}\right)$. (i) $\alpha_{1}(\lambda): u_{1} \mapsto u_{1}+\lambda u_{2}, u_{j} \mapsto u_{j}(j \neq 1)$, (ii) $\alpha_{2}(\lambda)$ : $u_{5} \mapsto u_{5}+\lambda u_{4}, u_{j} \mapsto u_{j}(j \neq 5)$, (iii) $\beta_{1}(\lambda): u_{1} \mapsto u_{1}+\lambda u_{4}, u_{3} \mapsto u_{3}+\lambda u_{2}, u_{j} \mapsto u_{j}$ $(j \neq 1,3)$ (iv) $\beta_{2}(\lambda): u_{3} \mapsto u_{3}+\lambda u_{4}, u_{5} \mapsto u_{5}+\lambda u_{2}, u_{j} \mapsto u_{j}(j \neq 3,5)$ (v) $\gamma_{1}(\lambda)$ : $u_{2} \mapsto u_{2}-\lambda u_{4}, u_{3} \mapsto u_{3}+\lambda u_{1}, u_{5} \mapsto u_{5}+2 \lambda u_{3}+\lambda^{2} u_{1}, u_{j} \mapsto u_{j}(j \neq 2,3,5)$ and $\left(x_{1}, x_{2}, x_{3}\right) \mapsto\left(x_{1}, \lambda x_{1}+x_{2}, x_{3}\right)$, (vi) $\gamma_{2}(\lambda): u_{1} \mapsto u_{1}-2 \lambda u_{3}+\lambda^{2} u_{5}, u_{3} \mapsto u_{3}-\lambda u_{5}$, $u_{4} \mapsto u_{4}+\lambda u_{2}, \quad u_{j} \mapsto u_{j}(j \neq 1,3,4)$ and $\left(x_{1}, x_{2}, x_{3}\right) \mapsto\left(x_{1}-\lambda x_{2}, x_{2}, x_{3}\right)$. We have also $\xi_{1}(\lambda)$ (resp. $\left.\xi_{2}(\lambda)\right):\left(x_{1}, x_{2}, x_{3}\right) \mapsto\left(x_{1}, x_{2}, \lambda x_{1}+x_{3}\right)$ (resp. $\left(x_{1}, x_{2}, \lambda x_{2}+\right.$ $\left.x_{3}\right)$ ) and $\eta(\mu):\left(x_{1}, x_{2}, x_{3}\right) \rightarrow\left(x_{1}, x_{2}, \mu x_{3}\right)$ with $\mu \neq 0$. By using these actions, we shall do the orbital decomposition leaving $\left(x_{1}, x_{2}\right)$ fixed. If at least one of $a_{13}, a_{15}$ and $a_{35}$ is not zero, then by $\gamma_{1}, \gamma_{2}, \xi_{1}, \xi_{2}$ and $\eta$, we may assume that $a_{13}=1, a_{34}=a_{35}=a_{45}=0$. (i) If $a_{15} \neq 0$, by $\alpha_{1}, \beta_{1}, \xi_{1}, \xi_{2}, \beta_{2}, \alpha_{2}, \gamma_{1}$ and $\eta$, we may assume that $x_{3}=a_{24} u_{2} \wedge u_{4}+u_{1} \wedge u_{5}$. If $a_{24} \neq 0$ (resp. $a_{24}=0$ ), we have (1) (resp. (2)). (ii) If $a_{15}=0$, by $\xi_{1}, \xi_{2}, \beta_{1}, \beta_{2}$ and $\alpha_{1}$, we may assume that $x_{3}=u_{1} \wedge u_{3}+a_{24} u_{2} \wedge u_{4}+a_{25} u_{2} \wedge u_{5}$. If $a_{25} \neq 0$, then we have (1). 
If $a_{25}=0$, then it is reduced to previous cases. Finally, if $a_{13}=a_{15}=a_{35}$ $=0$, we may assume that $a_{15}=a_{25}=a_{35}=a_{45}=0$ by the action of $\xi_{1}, \xi_{2}$ and $\gamma_{2}$. By considering $\left(x_{1}, x_{3}\right)$ instead of $\left(x_{1}, x_{2}\right)$, it is reduced to the previous cases. We shall see later, by calculating the isotropy subalgebras, that these orbits are different from each other.

Q.E.D.

(1) Put $x_{0}^{\prime}=\left(3 u_{3} \wedge u_{4}-u_{2} \wedge u_{5}, u_{1} \wedge u_{5}-2 u_{2} \wedge u_{4}, 3 u_{2} \wedge u_{3}-u_{1} \wedge u_{4}\right)$. Then the isotropy subalgebra $g_{x_{0}^{\prime}}$ is the following standard form.

$$
\begin{aligned}
\mathfrak{g}_{x_{0}^{\prime}}= & \left\{\left(\begin{array}{ccccc}
4 \alpha & \beta & & & \\
4 \gamma & 2 \alpha & 2 \beta & & \\
& 3 \gamma & 0 & 3 \beta & \\
& 2 \gamma & -2 \alpha & 4 \beta \\
& & \gamma & -4 \alpha
\end{array}\right) \oplus\left(\begin{array}{ccc}
2 \alpha & \beta & \\
2 \gamma & & 2 \beta \\
& \gamma & -2 \alpha
\end{array}\right)\right\} \\
& \cong\left\{\left(\begin{array}{rr}
\alpha & \beta \\
\gamma & -\alpha
\end{array}\right)\right\}=\operatorname{si}(2) .
\end{aligned}
$$

Since $\Lambda_{0}=V \times\{0\}$, we have $\operatorname{ord}_{V_{0}^{(0)}\left({ }_{0}\right.} f^{s}=0$ where $f$ denotes the relative invariant of degree 15 (See [1]).

(2) The isotropy subgroup at $x_{1}$ is locally isomorphic to $(G L(1) \times G L(1))$. $U(2)$ where $U(2)$ denotes a 2-dimensional unipotent group (See [1]). The conormal vector space $V_{x_{1}}^{*}$ is spanned by $\left(u_{3} \wedge u_{5}, 0,0\right) \in S_{21,1}^{*}$. We have $\operatorname{dim} \Lambda_{0} \cap \Lambda_{1}=\operatorname{dim} V-1 ; \operatorname{ord}_{\Lambda_{1}} f^{s}=-s-\frac{1}{2}$ and $b_{\Lambda_{1}}(s) / b_{\Lambda_{0}}(s)=(s+1)$.

(3) The isotropy subalgebra $g_{x_{2}}$ at $x_{2}$ is given as follows.

$$
\begin{aligned}
\mathfrak{g}_{x_{2}} & =\left\{A=\left(\begin{array}{ccccc}
\varepsilon & 0 & 0 & 0 & -\beta \\
\alpha & -2(\varepsilon+\eta) & \beta & 0 & 0 \\
0 & 0 & \eta & 0 & -\alpha \\
0 & 0 & -\beta & -2(\varepsilon+\eta) & \gamma \\
0 & 0 & 0 & 0 & 3(\varepsilon+\eta)
\end{array}\right)\right. \\
& \left.\oplus\left(\begin{array}{ccc}
\varepsilon+2 \eta & & \\
-\beta & -\alpha+\eta & -(\varepsilon+\eta)
\end{array}\right)\right\} \cong(\mathfrak{g l}(1) \oplus \mathfrak{g l}(1)) \oplus \mathfrak{u}(3) .
\end{aligned}
$$

The conormal vector space $V_{x_{2}}^{*}$ is spanned by $v_{1}=\left(u_{3} \wedge u_{5}, 0,0\right)$, and $v_{2}=\left(0, u_{1} \wedge u_{5}, 0\right)$, and the action $d \rho_{x_{2}}$ is given by

$$
d \rho_{x_{2}}(A)\left(v_{1}, v_{2}\right)=\left(v_{1}, v_{2}\right)\left(\begin{array}{cc}
-(4 \varepsilon+6 \eta) & 0 \\
0 & -(6 \varepsilon+4 \eta)
\end{array}\right)
$$


i) $V_{x_{2}}^{*}-S_{x_{2}}^{*} \leftrightarrow v_{1}+v_{2}=\left(u_{3} \wedge u_{5}, u_{1} \wedge u_{5}\right.$, 0$)$, i.e., $v_{1}+v_{2}$ is a generic point of $V_{x_{2}}^{*}$, where $S_{x_{2}}^{*}$ is the singular set of the P.V. $\left(G_{x_{2}}, \rho_{x_{2}}, V_{x_{2}}^{*}\right)$. We use this notation from now in $\S 11$. Put $y=y_{1} v_{1}+y_{2} v_{2}$.

ii) $\quad\left(S_{x_{2}}^{*}\right)_{1} \leftrightarrow d \rho_{1}(A)=-(4 \varepsilon+6 \eta) \leftrightarrow f_{1}^{*}(y)=y_{1} \leftrightarrow v_{2}=\left(0, u_{1} \wedge u_{5}, 0\right) \in S_{21,1}^{*}$, i.e., $\left(S_{x_{2}}^{*}\right)_{1}=\left\{y \in V_{x_{2}}^{*} ; f_{1}^{*}(y)=0\right\}=\overline{\rho_{x_{2}}\left(G_{x_{2}}\right) \cdot v_{2}}$ and $f_{1}^{*}\left(\rho_{x_{2}}(g) y\right)=\rho_{1}(g) f_{1}^{*}(y)$ for $y \in V_{x_{2}}^{*}, g \in G_{x_{2}}$. From now on, we use this notation in $\S 11$.

iii) $\quad\left(S_{x_{2}}^{*}\right)_{2} \leftrightarrow d \rho_{2}(A)=-(6 \varepsilon+4 \eta) \leftrightarrow f_{2}^{*}(y)=y_{2} \leftrightarrow v_{1}=\left(u_{3} \wedge u_{5}, 0,0\right) \in S_{21,1}^{*}$

iv) $-\delta \chi=d \rho_{1}+d \rho_{2}, \operatorname{tr}_{V_{x_{2}}^{*}}=d \rho_{1}+d \rho_{2}$

v) $\operatorname{ord}_{A_{2}} f^{s}=-2 s-2 / 2$.

Since the Hessian of the localization $f_{x_{2}}(z)=z_{1} z_{2}\left(z=z_{1} v_{1}+z_{2} v_{2} \in V_{x_{2}}\right)$ of $f(x)$ is not identically zero, $\Lambda_{2}=\Lambda_{2,16}^{(2)}$ is a good holonomic variety. We have $\operatorname{dim} \Lambda_{1} \cap \Lambda_{2}=\operatorname{dim} V-1$ and $b_{\Lambda_{2}}(s) / b_{\Lambda_{1}}(s)=(s+1)$.

(4) The isotropy subalgebra $\mathfrak{g}_{x_{3}}$ is given as follows.

(11.3)

$$
\mathfrak{g}_{x_{3}}=\left\{\begin{array}{ccccc}
\varepsilon & \alpha & 0 & 0 & \beta \\
0 & \eta & 0 & 0 & 0 \\
0 & 0 & \xi & 0 & 0 \\
0 & 0 & \gamma & \varepsilon & \beta \\
0 & 0 & 0 & 0 & -(2 \varepsilon+\eta+\xi)
\end{array}\right\}
$$

$$
\begin{aligned}
& \oplus\left(\begin{array}{lll}
-(\varepsilon+\eta) & & \\
& -(\varepsilon+\xi) & \\
& & (\varepsilon+\eta+\xi)
\end{array}\right) \\
& \cong(\mathfrak{g r}(1) \oplus \mathfrak{g r}(1) \oplus \operatorname{gr}(1)) \oplus \mathfrak{u}(3) .
\end{aligned}
$$

The conormal vector space $V_{x_{3}}^{*}$ is spanned by $v_{1}=\left(u_{3} \wedge u_{5}, 0,0\right), v_{2}=(0$, $\left.u_{2} \wedge u_{5}, 0\right) v_{3}=\left(0,0, u_{2} \wedge u_{3}\right)$, and

$$
d \rho_{x_{3}}(A)\left(v_{1}, v_{2}, v_{3}\right)=\left(v_{1}, v_{2}, v_{3}\right)\left(\begin{array}{lll}
3 \varepsilon+2 \eta & & \\
& 3 \varepsilon+2 \xi & \\
& & -(\varepsilon+2 \eta+2 \xi)
\end{array}\right)
$$

i) $\quad V_{x_{3}}^{*}-S_{x_{3}}^{*} \leftrightarrow v_{1}+v_{2}+v_{3}=\left(u_{3} \wedge u_{5}, u_{2} \wedge u_{5}, u_{2} \wedge u_{3}\right) \in S_{15,3}^{*}$

ii) $\left(S_{x_{3}}^{*}\right)_{1} \leftrightarrow d \rho_{1}(A)=3 \varepsilon+2 \eta \leftrightarrow f_{1}^{*}(y)=y_{1} \leftrightarrow v_{2}+v_{3}=\left(0, u_{2} \wedge u_{5}, u_{2} \wedge u_{3}\right)$ $\in S_{16,2}^{*}$

iii) $\left(S_{x_{3}}^{*}\right)_{2} \leftrightarrow d \rho_{2}(A)=3 \varepsilon+2 \xi \leftrightarrow f_{2}^{*}(y)=y_{2} \leftrightarrow v_{1}+v_{3}=\left(u_{3} \wedge u_{5}, 0, u_{2} \wedge u_{3}\right)$ $\in S_{18,2}^{*}$

iv) $\left(S_{x_{3}}^{*}\right)_{3} \leftrightarrow d \rho_{3}(A)=-(\varepsilon+2 \eta+2 \xi) \leftrightarrow f_{3}^{*}(y)=y_{3} \leftrightarrow v_{1}+v_{2}=\left(u_{3} \wedge u_{5}\right.$, $\left.u_{2} \wedge u_{5}, 0\right) \in S_{16,2}^{*}$

v) $-\delta \chi=d \rho_{1}+d \rho_{2}+d \rho_{3}, \operatorname{tr}_{v_{x_{3}}^{*}}=d \rho_{1}+d \rho_{2}+d \rho_{3}$. 
Since the localization $f_{x_{3}}(z)=z_{1} z_{2} z_{3} \quad\left(z=\sum z_{i} v_{i} \in V_{x_{3}}\right)$ is non-degenerate, $\Lambda_{3,15}$ is a good holonomic variety and $\operatorname{ord}_{A_{3}, 15} f^{s}=-3 s-\frac{3}{2}$. We have $\operatorname{dim} \Lambda_{2} \cap \Lambda_{3,15}=\operatorname{dim} V-1$ and $b_{\Lambda_{3}, 15}(s) / b_{\Lambda_{2}}(s)=(s+1)$.

(5) The isotropy subalgebra $\mathfrak{g}_{x^{\prime}}$ at $x_{3}^{\prime}$ is given as follows.

$$
\begin{aligned}
\mathfrak{g}_{x_{3}^{\prime}}= & \left\{A=\left(\begin{array}{ccccc}
-2(\varepsilon+\eta) & \alpha & \beta & 0 & 0 \\
0 & \varepsilon & \gamma & 0 & -2 \alpha \\
0 & 0 & \eta & 0 & 0 \\
0 & 0 & -\alpha & -(3 \varepsilon+\eta) & \delta \\
0 & 0 & 0 & 0 & 4 \varepsilon+2 \eta
\end{array}\right]\right. \\
& \left.\oplus\left(\begin{array}{ccc}
\varepsilon+2 \eta & 0 & 0 \\
-\gamma & 2 \varepsilon+\eta & 0 \\
\beta & -\alpha & -\varepsilon-\eta
\end{array}\right)\right\} \cong(\operatorname{gr}(1) \oplus \operatorname{gr}(1)) \oplus \mathfrak{u}(4) .
\end{aligned}
$$

The conormal vector space $V_{x^{\prime}}^{*}$ is spanned by $v_{1}=\left(u_{2} \wedge u_{5},-u_{3} \wedge u_{5}, 0\right)$, $v_{2}=\left(u_{3} \wedge u_{4}, 0,0\right), v_{3}=\left(u_{3} \wedge u_{5}, 0,0\right)$, and

$$
d \rho_{x_{3}}(A)\left(v_{1}, v_{2}, v_{3}\right)=\left(v_{1}, v_{2}, v_{3}\right)\left(\begin{array}{ccc}
-(6 \varepsilon+4 \eta) & 0 & 0 \\
0 & 2(\varepsilon-\eta) & 0 \\
-2 \gamma & -\delta & -5(\varepsilon+\eta)
\end{array}\right)
$$

i) $V_{x_{3^{\prime}}}^{*}-S_{x_{3^{\prime}}}^{*} \leftrightarrow v_{1}+v_{2}=\left(u_{2} \wedge u_{5}+u_{3} \wedge u_{4},-u_{3} \wedge u_{5}, 0\right) \in S_{13,3}^{*}$

ii) $\left(S_{x^{\prime}}^{*}\right)_{1} \leftrightarrow d \rho_{1}(A)=-(6 \varepsilon+4 \eta) \leftrightarrow f_{1}^{*}(y)=y_{1} \leftrightarrow v_{2} \in S_{21,1}^{*}$

iii) $\left(S_{x_{3}^{\prime}}^{*}\right)_{2} \leftrightarrow d \rho_{2}(A)=2(\varepsilon-\eta) \leftrightarrow f_{2}^{*}(y)=y_{2} \leftrightarrow v_{1} \in S_{16,2}^{*}$

iv) $-\delta \chi=2 d \rho_{1}+d \rho_{2}=-10(\varepsilon+\eta), \operatorname{tr}_{V_{x_{3}}^{*}}=2 d \rho_{1}+\frac{3}{2} d \rho_{2}=-9 \varepsilon-11 \eta$.

Since $\operatorname{dim} \Lambda_{3,13} \cap \Lambda_{2}=\operatorname{dim} \Lambda_{3,13} \cap \Lambda_{1}=\operatorname{dim} V-1$ and they intersect $G_{0^{-}}$ prehomogeneously, $\Lambda_{3,13}$ is a good holonomic variety and $\operatorname{ord}_{\Lambda_{3,13}} f^{s}=-3 s$ - $\frac{4}{2}$. The intersection exponent of $\Lambda_{3,13}$ and $\Lambda_{1}$ is $(1: 0)$. We have $b_{A_{3}, 13}(s) /$ $b_{\Lambda_{1}}(s)=(s+1)\left(s+\frac{3}{2}\right)$ and $b_{\Lambda_{3}, 13}(s) / b_{\Lambda_{2}}(s)=\left(s+\frac{3}{2}\right)$.

(6) The isotropy subalgebra $\mathfrak{g}_{x_{4}}$ at $x_{4}$ is given as follows.

$$
\begin{aligned}
\mathfrak{g}_{x_{4}}= & \left\{\begin{array}{ccccc}
A & \alpha & \gamma & 0 & 0 \\
0 & \eta & \beta & 0 & 0 \\
0 & 0 & \xi & 0 & 0 \\
0 & 0 & -\alpha & \varepsilon-\eta+\xi & \delta \\
0 & 0 & 0 & 0 & -2(\varepsilon+\xi)
\end{array}\right) \\
& \left.\oplus\left(\begin{array}{ccc}
-(\varepsilon+\eta) & 0 & 0 \\
-\beta & -(\varepsilon+\xi) & 0 \\
0 & 0 & (\varepsilon+\eta+\xi)
\end{array}\right)\right\} \cong(\mathfrak{g r}(1) \oplus \mathfrak{g l}(1) \oplus \mathfrak{g l}(1)) \oplus \mathfrak{H}(4) .
\end{aligned}
$$


The conormal vector space $V_{x_{4}}^{*}$ is spanned by $v_{1}=\left(u_{3} \wedge u_{4}, 0,0\right), v_{2}=\left(u_{2}\right.$ $\left.\wedge u_{5},-u_{3} \wedge u_{5}, 0\right), v_{3}=\left(u_{3} \wedge u_{5}, 0, u_{2} \wedge u_{3}\right), v_{4}=\left(0,0, u_{2} \wedge u_{3}\right)$, and

$d \rho_{x_{4}}(A)\left(v_{1}, \cdots, v_{4}\right)=\left(v_{1}, \cdots, v_{4}\right)\left(\begin{array}{cccc}2(\eta-\varepsilon) & 0 & 0 & 0 \\ 0 & 3 \varepsilon+2 \eta & 0 & 0 \\ \alpha & -2 \beta & 3 \varepsilon+\eta+\xi & 0 \\ 0 & 0 & 0 & -(\varepsilon+2 \eta+2 \xi)\end{array}\right)$

i) $\quad V_{x_{4}}^{*}-S_{x_{4}}^{*} \leftrightarrow v_{1}+v_{2}+v_{4}=\left(u_{3} \wedge u_{4}+u_{2} \wedge u_{5},-u_{3} \wedge u_{5}, u_{2} \wedge u_{3}\right) \in S_{11,4}^{*}$

ii) $\left(S_{x_{4}}^{*}\right)_{1} \leftrightarrow d \rho_{1}(A)=2(\eta-\xi) \leftrightarrow f_{1}^{*}(y)=y_{1} \leftrightarrow v_{2}+v_{4}$ $=\left(u_{2} \wedge u_{5},-u_{3} \wedge u_{5}, u_{2} \wedge u_{3}\right) \in S_{15,3}^{*}$

iii) $\left(S_{x_{4}}^{*}\right)_{2} \leftrightarrow d \rho_{2}(A)=3 \varepsilon+2 \xi \leftrightarrow f_{2}^{*}(y)=y_{2} \leftrightarrow v_{1}+v_{4}$ $=\left(u_{3} \wedge u_{4}, 0, u_{2} \wedge u_{3}\right) \in S_{16,2}^{*}$

iv) $\left(S_{x_{4}}^{*}\right)_{3} \leftrightarrow d \rho_{3}(A)=-(\varepsilon+2 \eta+2 \xi) \leftrightarrow f_{3}^{*}(y)=y_{4} \leftrightarrow v_{1}+v_{2}$ $=\left(u_{2} \wedge u_{5}+u_{3} \wedge u_{4},-u_{3} \wedge u_{5}, 0\right) \in S_{13,3}^{*}$

v) $-\delta \chi=d \rho_{1}+2 d \rho_{2}+d \rho_{3}, \operatorname{tr}_{V x_{4}}^{*}=\frac{3}{2} d \rho_{1}+2 d \rho_{2}+d \rho_{3}$.

The conormal bundle $\Lambda_{4}$ is a good holonomic variety with $\operatorname{ord}_{\Lambda_{4}} f^{s}=-4 s$ $-\frac{5}{2}$. We have $b_{A_{4}}(s) / b_{A_{2}}(s)=(s+1)\left(s+\frac{3}{2}\right), \quad b_{A_{4}}(s) / b_{A_{3}, 13}(s)=(s+1)$ and $b_{4_{4}}(s) / b_{A_{3}, 15}(s)=\left(s+\frac{3}{2}\right)$. Note that these intersections are regular and $G_{0^{-}}$ prehomogeneous.

(7) The isotropy subalgebra $g_{x_{5}}$ at $x_{5}$ is given as follows.

$$
\begin{aligned}
& \mathfrak{g}_{x_{5}}=\left\{\begin{array}{ccccc}
\varepsilon & \gamma_{1} & \gamma_{2} & \gamma_{3} & \gamma_{4} \\
0 & 2 \varepsilon+4 \eta & \gamma_{5} & 0 & \gamma_{6} \\
0 & 0 & \eta & 0 & \gamma_{3}+\gamma_{5} \\
0 & 0 & -\gamma_{1} & -(\varepsilon+3 \eta) & -\gamma_{2} \\
0 & 0 & 0 & 0 & -2(\varepsilon+\eta)
\end{array}\right) \\
& \left.\oplus\left(\begin{array}{ccc}
-(3 \varepsilon+4 \eta) & 0 & 0 \\
\gamma_{3}-\gamma_{5} & -(\varepsilon+\eta) & 0 \\
-\gamma_{6} & -\gamma_{5} & \varepsilon+2 \eta
\end{array}\right)\right\} \cong(\mathfrak{g l}(1) \oplus \operatorname{gr}(1)) \oplus \mathfrak{l t}(6) \text {. }
\end{aligned}
$$

Then $V_{x_{5}}^{*}$ is spanned by $v_{1}=\left(u_{1} \wedge u_{5}-u_{3} \wedge u_{4}, u_{4} \wedge u_{5}, 0\right), v_{2}=\left(u_{2} \wedge u_{3}\right.$, $\left.-u_{2} \wedge u_{5}, u_{3} \wedge u_{5}\right), v_{3}=\left(u_{2} \wedge u_{5},-u_{3} \wedge u_{5}, 0\right), v_{4}=\left(u_{4} \wedge u_{5}, 0,0\right), v_{5}=\left(u_{3}\right.$ $\left.\wedge u_{5}, 0,0\right)$, and

$$
d \rho_{x_{5}}(A)\left(v_{1}, \cdots, v_{5}\right)=\left(v_{1}, \cdots, v_{5}\right)\left(\begin{array}{ccccc}
4 \varepsilon+6 \eta & 0 & 0 & 0 & 0 \\
0 & \varepsilon-\eta & 0 & 0 & 0 \\
-\gamma_{1} & -\gamma_{5} & 3 \varepsilon+2 \eta & 0 & 0 \\
-3 \gamma_{3} & 0 & 0 & 6 \varepsilon+9 \eta & 0 \\
-2 \gamma_{5} & 2 \gamma_{6} & \gamma_{3}-2 \gamma_{5} & \gamma_{1} & 5(\varepsilon+\eta)
\end{array}\right)
$$


i) $\quad V_{x_{5}}^{*}-S_{x_{5}}^{*} \leftrightarrow v_{1}+v_{2}=\left(u_{1} \wedge u_{5}-u_{3} \wedge u_{4}+u_{2} \wedge u_{3}, u_{4} \wedge u_{5}\right.$ $\left.-u_{2} \wedge u_{5}, u_{3} \wedge u_{5}\right) \in S_{8,5}^{*}$

ii) $\left(S_{x_{5}}^{*}\right)_{1} \leftrightarrow d \rho_{1}(A)=4 \varepsilon+6 \eta \leftrightarrow f_{1}^{*}(y)=y_{1} \leftrightarrow v_{2}+v_{4} \in S_{11,4}^{*}$

iii) $\left(S_{x_{5}}^{*}\right)_{2} \leftrightarrow d \rho_{2}(A)=\varepsilon-\eta \leftrightarrow f_{2}^{*}(y)=y_{2} \leftrightarrow v_{1} \in S_{13,3}^{*}$

iv) $-\delta \chi=3 d \rho_{1}+3 d \rho_{2}(=15 \varepsilon+15 \eta), \operatorname{tr}_{V_{x_{5}}^{*}}=4 d \rho_{1}+3 d \rho_{2}(=19 \varepsilon+21 \eta)$.

The conormal bundle $\Lambda_{5}$ is a good holonomic variety with $\operatorname{ord}_{\Lambda_{5}} f^{s}=-6 s$ $-\frac{9}{2}$. We have $b_{A_{5}}(s) / b_{\Lambda_{4}}(s)=\left(s+\frac{4}{3}\right)\left(s+\frac{5}{3}\right)$ and $b_{\Lambda_{5}}(s) / b_{A_{3,13}}(s)=(s+1)\left(s+\frac{4}{3}\right)$ $\left(s+\frac{5}{3}\right)$. Note that the intersection exponent of $\Lambda_{5}$ and $\Lambda_{4}$ is $(2: 1)$. The intersection of $\Lambda_{5}$ and $\Lambda_{3,13}$ is regular and $G_{0}$-prehomogeneous.

(8) The isotropy subalgebra $g_{x_{6}}$ at $x_{6}$ is given as follows.

$$
\begin{aligned}
\mathfrak{g}_{x_{6}}= & \left\{\tilde{A}=\left(\begin{array}{c|c|c}
-4 \varepsilon & \frac{\gamma_{1} \gamma_{2}}{0} & \frac{0}{\varepsilon I_{2}+A} \\
\hline 0 & \frac{0}{0} & \frac{0}{\varepsilon I_{2}+B}
\end{array}\right) \oplus\left(\frac{3 \varepsilon I_{2}-{ }^{t} A}{\gamma_{2}-\gamma_{1}} \mid \frac{0}{-2 \varepsilon}\right) ; A, B \in \mathfrak{g l}(2)\right\} \\
& \cong(\mathfrak{g l}(1) \oplus \operatorname{gl}(2) \oplus \mathfrak{g l}(2)) \oplus V(2) .
\end{aligned}
$$

Then $V_{x_{6}}^{*}$ is spanned by $v_{1}=\left(u_{2} \wedge u_{4},-u_{3} \wedge u_{4}, 0\right), v_{2}=\left(0, u_{2} \wedge u_{4}, 0\right), v_{3}=$ $\left(u_{3} \wedge u_{4}, 0,0\right), v_{4}=\left(u_{2} \wedge u_{5},-u_{3} \wedge u_{5}, 0\right), v_{5}=\left(0, u_{2} \wedge u_{5}, 0\right), v_{6}=\left(u_{3} \wedge u_{5}\right.$, $0,0)$, and $\left(G_{x_{6}}, \rho_{x_{6}}, V_{x_{6}}^{*}\right) \cong\left(G L(1) \times S L(2) \times S L(2), 5 \Lambda_{1} \otimes 2 \Lambda_{1} \otimes \Lambda_{1}, V(1) \otimes V(3)\right.$ $\otimes V(2)) \cong\left(S O(3) \times G L(2), \Lambda_{1} \otimes \Lambda_{1}, V(3) \otimes V(2)\right)$ and hence $\Lambda_{6}$ is a good holonomic variety.

i) $V_{x_{6}}^{*}-S_{x_{6}}^{*} \leftrightarrow v_{2}+v_{6}=\left(u_{3} \wedge u_{5}, u_{2} \wedge u_{4}, 0\right) \in S_{12,6}^{*}$

ii) $\left(S_{x_{6}}^{*}\right)_{1} \leftrightarrow v_{2}+v_{4}=\left(u_{2} \wedge u_{5}, u_{2} \wedge u_{4}-u_{3} \wedge u_{5}, 0\right) \in S_{13,3}^{*}$

iii) $-\delta \chi=d \rho_{1}, \operatorname{tr}_{V_{x_{6}}^{*}}=\frac{3}{2} d \rho_{1}$

We have $\operatorname{ord}_{A_{6}} f^{s}=-4 s-\frac{6}{2}$ and $b_{A_{6}}(s) / b_{A_{3}, 13}(s)=\left(s+\frac{3}{2}\right)$.

(9) The isotropy subalgebra $\mathfrak{g}_{x_{7}}$ at $x_{7}$ is given as follows.

$$
\begin{aligned}
& g_{x_{7}}=\left\{\tilde{A}=\left(\begin{array}{c|c|c}
-2(\varepsilon+\eta) & F & 0 \\
\hline 0 & \frac{\varepsilon I_{2}+A}{0} & 0 \\
\hline 0 & \eta I_{2}+B
\end{array}\right) \oplus\left(\begin{array}{c|c}
(\varepsilon+2 \eta) I_{2}-{ }^{t} A & 0 \\
\hline 0 & -2 \eta
\end{array}\right)\right. \\
& \left.A, B \in \mathfrak{g l}(2),{ }^{t} F \in C^{2}\right\} \cong(\mathfrak{g l}(1) \oplus \operatorname{gr}(1) \oplus \mathfrak{g l}(2) \oplus \mathfrak{g}(2)) \oplus V(2) \text {. }
\end{aligned}
$$

Then $V_{x_{7}}^{*}$ is spanned by $v_{1}=\left(u_{2} \wedge u_{4},-u_{3} \wedge u_{4}, 0\right), v_{2}=\left(0, u_{2} \wedge u_{4}, 0\right), v_{3}$ $=\left(u_{3} \wedge u_{4}, 0,0\right), v_{4}=\left(u_{2} \wedge u_{5},-u_{3} \wedge u_{5}, 0\right), v_{5}=\left(0, u_{2} \wedge u_{5}, 0\right), v_{6}=\left(u_{3} \wedge\right.$ $\left.u_{5}, 0,0\right), \quad v_{7}=\left(0,0, u_{2} \wedge u_{3}\right)$, and $\left(G_{x_{7}}, \rho_{x_{7}}, V_{x_{7}}^{*}\right) \cong(G L(1) \times G L(1) \times S L(2) \times$ $\left.S L(2),\left(2 \Lambda_{1}^{*} \otimes 3 \Lambda_{1}^{*} \otimes 2 \Lambda_{1} \otimes \Lambda_{1}\right) \oplus\left(2 \Lambda_{1}^{*} \otimes 2 \Lambda_{1} \otimes 1 \otimes 1\right), V(6) \oplus V(1)\right)$.

i) $\quad V_{x_{7}}^{*}-S_{x_{7}}^{*} \leftrightarrow v_{2}+v_{6}+v_{7}=\left(u_{3} \wedge u_{5}, u_{2} \wedge u_{4}, u_{2} \wedge u_{3}\right) \in S_{9,7}^{*}$

ii) $\left(S_{x_{7}}^{*}\right)_{1} \leftrightarrow d \rho_{1}(\tilde{A})=-8 \varepsilon-12 \eta \leftrightarrow f_{1}^{*}(y)\left(\operatorname{deg} f_{1}^{*}=4\right) \leftrightarrow v_{2}+v_{4}+v_{7} \in S_{11,4}^{*}$ 
iii) $\left(S_{x_{7}}^{*}\right)_{2} \leftrightarrow d \rho_{2}(\tilde{A})=-2 \varepsilon+2 \eta \leftrightarrow f_{2}^{*}(y)=y_{7} \leftrightarrow v_{2}+v_{6} \in S_{12,6}^{*}$

iv) $-\delta \chi=d \rho_{1}+d \rho_{2}, \operatorname{tr}_{V_{x_{7}}^{*}}=\frac{3}{2} d \rho_{1}+d \rho_{2}$.

Then by Corollary 1-7 conormal bundle $\Lambda_{7,9}$ is a good holonomic variety with $\operatorname{ord}_{\Lambda_{7}, 9} f^{s}=-5 s-\frac{7}{2}$. We have $\operatorname{dim} \Lambda_{7,9} \cap \Lambda_{4}=\operatorname{dim} \Lambda_{7,9} \cap \Lambda_{6}=\operatorname{dim} V$ $-1, b_{A_{7}, 9}(s) / b_{A_{4}}(s)=\left(s+\frac{3}{2}\right)$, and $b_{A_{7}, 9}(s) / b_{A_{6}}(s)=(s+1)$.

(10) The isotropy subalgebra $g_{x_{7}^{\prime}}$ at $x_{7}^{\prime}$ is given as follows.

$$
\begin{aligned}
\mathfrak{g}_{x_{7}^{\prime}}= & \left\{A=\left(\begin{array}{cc|ccc}
3 \varepsilon+\alpha & \beta & \gamma_{1} & \gamma_{2} & \gamma_{3} \\
\gamma & 3 \varepsilon-\alpha & \gamma_{4} & \gamma_{5} & \gamma_{6} \\
\hline & 0 & -2 \varepsilon & -2 \gamma & -2 \beta \\
& 0 & -\beta & -2 \varepsilon+2 \alpha & 0 \\
-\gamma & 0 & -2 \varepsilon-2 \alpha
\end{array}\right]\right. \\
& \left.\oplus\left(\begin{array}{c|c|c}
-6 \varepsilon & 0 & \\
\hline \gamma_{2}-\gamma_{4} & -\varepsilon-\alpha & \gamma \\
\gamma_{1}-\gamma_{6} & \beta & -\varepsilon+\alpha
\end{array}\right)\right\} \cong(\mathfrak{g l}(1) \oplus \operatorname{grl}(2)) \oplus V(6) .
\end{aligned}
$$

Then $V_{x_{7^{\prime}}}^{*}$ is spanned by $v_{1}=\left(u_{1} \wedge u_{4}, 0, u_{3} \wedge u_{4}\right), v_{2}=\left(u_{1} \wedge u_{3}-u_{2} \wedge u_{4}\right.$, $\left.-u_{3} \wedge u_{4},-2 u_{4} \wedge u_{5}\right), \quad v_{3}=\left(u_{1} \wedge u_{5}-u_{2} \wedge u_{3}, 2 u_{4} \wedge u_{5},-u_{3} \wedge u_{5}\right), \quad v_{4}=$ $\left(-u_{2} \wedge u_{5}, u_{3} \wedge u_{5}, 0\right), v_{5}=\left(u_{4} \wedge u_{5}, 0,0\right), v_{6}=\left(u_{3} \wedge u_{4}, 0,0\right), v_{7}=\left(u_{3} \wedge u_{5}\right.$, $0,0)$ and the action $d \rho_{x_{\gamma}^{\prime}}$ of $g_{x_{7}^{\prime}}$ on $V_{x_{7}^{\prime}}^{*}$ is given by

$$
d \rho_{x_{7}}(A)\left(v_{1}, \cdots, v_{7}\right)=\left(v_{1}, \cdots, v_{7}\right)\left(\frac{5 \varepsilon I_{4}+A_{1}}{C} \mid \frac{0}{10 \varepsilon I_{3}+A_{2}}\right)
$$

where

$$
\left(C, A_{2}\right)=\left(\begin{array}{ccccccc}
\gamma_{3} & 2 \gamma_{1}-\gamma_{6} & 3 \gamma_{2}-2 \gamma_{4} & -\gamma_{5} & 0 & -2 \beta & 2 \gamma \\
\gamma_{6}-2 \gamma_{1} & 2 \gamma_{2} & \gamma_{5} & 0 & -\gamma & -2 \alpha & 0 \\
0 & \gamma_{3} & 2 \gamma_{6} & \gamma_{2}-2 \gamma_{4} & \beta & 0 & 2 \alpha
\end{array}\right)
$$

and

$$
A_{1}=\left(\begin{array}{cccc}
-3 \alpha & 3 \gamma & 0 & 0 \\
\beta & -\alpha & -2 \gamma & 0 \\
0 & -2 \beta & \alpha & \gamma \\
0 & 0 & 3 \beta & 3 \alpha
\end{array}\right)
$$

i) $V_{x_{7^{\prime}}}^{*}-S_{x^{\prime}}^{*} \leftrightarrow v_{1}+v_{4}=\left(u_{1} \wedge u_{4}-u_{2} \wedge u_{5}, u_{3} \wedge u_{5}, u_{3} \wedge u_{4}\right) \in S_{7,7}^{(2) *}$

ii) $\left(S_{x_{7}}^{*}\right)_{1} \leftrightarrow d \rho_{1}(A)=20 \varepsilon \leftrightarrow f_{1}^{*}\left(y_{1}, \cdots, y_{4}\right)$ : the discriminant of binary cubic forms $\leftrightarrow v_{2} \in S_{8,5}^{*}$

iii) $-\delta \chi=2 d \rho_{1}, \operatorname{tr}_{V_{x 7^{\prime}}^{*}}=\frac{5}{2} d \rho_{1}$. 
The conormal bundle $\Lambda_{7,7}^{(1)}$ is a good holonomic variety with $\operatorname{ord}_{V_{7,7}^{(1)}} f^{s}=-$ $8 s-\frac{13}{2}$. We have $\operatorname{dim} \Lambda_{5} \cap \Lambda_{7,7}^{(1)}=\operatorname{dim} V-1$ and $b_{\Lambda_{7,7}^{(1)}}(s) / b_{\Lambda_{5}}(s)=\left(s+\frac{5}{4}\right)$ $\left(s+\frac{7}{4}\right)$. The intersection is regular and $G_{0}$-prehomogeneous.

(11) The isotropy subalgebra $g_{x_{7}^{\prime \prime}}$ at $x_{7}^{\prime \prime}$ is given as follows.

$$
\begin{aligned}
& \mathfrak{g}_{x_{i}^{\prime \prime}}=\left\{\tilde{A}=\left(\begin{array}{c|c|c}
-2(\varepsilon+\eta) & 0 & C \\
\hline 0 & \varepsilon I_{2}+A & D \\
\hline 0 & 0 & \eta I_{2}-{ }^{t} A
\end{array}\right) \oplus\left(\begin{array}{c|c}
(\varepsilon+2 \eta) I_{2}-{ }^{t} A & 0 \\
\hline C & -(\varepsilon+\eta)
\end{array}\right) ;\right. \\
& A \in \mathfrak{g l}(2)\} \cong(\mathfrak{g l}(1) \oplus \mathfrak{g l}(1) \oplus \mathfrak{g l}(2)) \oplus \mathfrak{u}(5) \text {. }
\end{aligned}
$$

Then $V_{x_{7}^{\prime \prime}}^{*}$ is spanned by $v_{1}=\left(u_{3} \wedge u_{4}, 0,0\right), v_{2}=\left(u_{2} \wedge u_{4}-u_{3} \wedge u_{5},-u_{3}\right.$ $\left.\wedge u_{4}, 0\right), v_{3}=\left(u_{2} \wedge u_{5}, u_{2} \wedge u_{4}-u_{3} \wedge u_{5}, 0\right), v_{4}=\left(0, u_{2} \wedge u_{5}, 0\right), v_{5}=\left(u_{1} \wedge u_{5}\right.$, $\left.-u_{1} \wedge u_{4}, u_{4} \wedge u_{5}\right), v_{6}=\left(u_{4} \wedge u_{5}, 0,0\right), v_{7}=\left(0, u_{4} \wedge u_{5}, 0\right)$, and $d \rho_{x_{7}^{\prime \prime}}(\tilde{A})\left(v_{1}, \cdots, v_{7}\right)=\left(v_{1}, \cdots, v_{7}\right)\left(\begin{array}{c|c|c}-(2 \varepsilon+3 \eta) I_{4}+3 \Lambda_{1}(A) & 0 & 0 \\ \hline 0 & \varepsilon-\eta & 0 \\ \hline * & * & -(\varepsilon+4 \eta) I_{2}+A\end{array}\right)$.

i) $V_{x_{7^{\prime \prime}}}^{*}-S_{x_{7^{\prime \prime}}}^{*} \leftrightarrow v_{1}+v_{4}+v_{5} \in S_{7,7}^{(1) *}$

ii) $\left(S_{x_{7}^{\prime \prime}}^{*}\right)_{1} \leftrightarrow d \rho_{1}(\tilde{A})=-8 \varepsilon-12 \eta \leftrightarrow f_{1}^{*}\left(y_{1}, \cdots, y_{4}\right)$ : the discriminant of binary cubic forms $\leftrightarrow v_{3}+v_{4}+v_{5} \in S_{8,5}^{*}$

iii) $\quad\left(S_{x^{\prime \prime}}^{*}\right)_{2} \leftrightarrow d \rho_{2}(\tilde{A})=\varepsilon-\eta \leftrightarrow f_{2}^{*}(y)=y_{5} \leftrightarrow v_{1}+v_{4} \in S_{12,6}^{*}$

iv) $-\delta \chi=d \rho_{1}+3 d \rho_{2}, \operatorname{tr}_{V x_{7}^{\prime \prime}}=\frac{3}{2} d \rho_{1}+3 d \rho_{2}$.

The conormal bundle $\Lambda_{7,7}^{(2)}$ is a good holonomic variety with $\operatorname{ord}_{\Lambda_{7}^{(2), 7}} f^{s}=-$ $7 s-\frac{11}{2}$. We have $\operatorname{dim} \Lambda_{5} \cap \Lambda_{7,7}^{(2)}=\operatorname{dim} \Lambda_{6} \cap \Lambda_{7,7}^{(2)}=\operatorname{dim} V-1, b_{\Lambda_{7}^{(2)}(s) / b_{\Lambda_{5}}}(s)=$ $\left(s+\frac{3}{2}\right)$ and $b_{A_{7}^{2}, 7}(s) / b_{A_{6}}(s)=(s+1)\left(s+\frac{4}{3}\right)\left(s+\frac{5}{3}\right)$. The intersections are regular and $G_{0}$-prehomogeneous.

(12) We shall calculate the isotropy subalgebra at $\tilde{x}_{8}=\left(u_{2} \wedge u_{3}+\right.$ $\left.u_{1} \wedge u_{4}, u_{1} \wedge u_{3}+u_{2} \wedge u_{5}, 0\right)$ instead of $x_{8}$.

$$
\begin{aligned}
\mathfrak{g}_{\tilde{x}_{8}}= & \left\{\tilde{A}=\left(\begin{array}{cc|ccc}
3 \varepsilon+\alpha & \alpha_{12} & \gamma_{1} & \gamma_{2} & \gamma_{3} \\
\alpha_{21} & 3 \varepsilon-\alpha & \gamma_{3} & \gamma_{1} & \gamma_{4} \\
\hline & 0 & -2 \varepsilon & -2 \alpha_{12} & -2 \alpha_{21} \\
-\alpha_{21} & -2 \varepsilon-2 \alpha & \\
-\alpha_{12} & & -2 \varepsilon+2 \alpha
\end{array}\right]\right. \\
& \left.\oplus\left(\begin{array}{cc|c}
-\varepsilon+\alpha & \alpha_{12} & \gamma_{5} \\
\frac{\alpha_{21}}{2}-\varepsilon-\alpha & \alpha_{6} \\
\hline 0 & \eta
\end{array}\right)\right\} \cong(\mathfrak{g r}(1) \oplus \mathfrak{g l}(1) \oplus \mathfrak{g l}(2)) \oplus V(6) .
\end{aligned}
$$


Then $V_{\tilde{x}_{8}}^{*}$ is spanned by $v_{1}=\left(0,0, u_{1} \wedge u_{2}\right), \quad v_{2}=\left(0,0, u_{1} \wedge u_{5}\right), v_{3}=(0,0$, $\left.u_{1} \wedge u_{3}-u_{2} \wedge u_{5}\right), v_{4}=\left(0,0, u_{1} \wedge u_{4}-u_{2} \wedge u_{3}\right), v_{5}=\left(0,0, u_{2} \wedge u_{4}\right), v_{6}=(0,0$, $\left.u_{4} \wedge u_{5}\right), v_{7}=\left(0,0, u_{3} \wedge u_{5}\right), v_{8}=\left(0,0, u_{3} \wedge u_{4}\right)$ and the action $d \rho_{\tilde{x}_{3}}$ of $g_{\tilde{x}_{8}}$ on $V_{\tilde{x}_{8}}^{*}$ is given by

$$
d \rho_{\tilde{x}_{8}}(\tilde{A})\left(v_{1}, \cdots, v_{8}\right)=\left(v_{1}, \cdots, v_{8}\right)\left(\begin{array}{c|c|c}
-6 \varepsilon-\eta & 0 & 0 \\
\hline C_{1} & -(\varepsilon+\eta) I_{4}+A_{1} & 0 \\
\hline 0 & C_{2} & \}_{1}\right\}_{3} \\
\}_{4}^{4} \\
\}_{3}
\end{array}\right.
$$

where

$$
\left(C_{2}, A_{2}\right)=\left(\begin{array}{rrrcccc}
-\gamma_{2} & \gamma_{1} & \gamma_{3} & \gamma_{4} & 0 & 2 \alpha_{12} & -2 \alpha_{21} \\
-\gamma_{1} & 2 \gamma_{3} & -\gamma_{4} & 0 & \alpha_{21} & -2 \alpha & 0 \\
0 & \gamma_{2} & -2 \gamma_{1} & -\gamma_{3} & -\alpha_{12} & 0 & 2 \alpha
\end{array}\right)
$$

and

$$
\left(C_{1}, A_{1}\right)=\left(\begin{array}{ccccc}
-\gamma_{4} & -3 \alpha & 3 \alpha_{21} & 0 & 0 \\
-\gamma_{3} & \alpha_{12} & -\alpha & 2 \alpha_{21} & 0 \\
-\gamma_{1} & 0 & 2 \alpha_{12} & \alpha & -\alpha_{21} \\
\gamma_{2} & 0 & 0 & -3 \alpha_{12} & 3 \alpha
\end{array}\right) .
$$

i) $\quad V_{\tilde{x}_{8}}^{*}-S_{\tilde{x}_{8}}^{*} \leftrightarrow v_{1}+v_{6}=\left(0,0, u_{1} \wedge u_{2}+u_{4} \wedge u_{5}\right) \in S_{18,8}^{*}$

ii) $\left(S_{\tilde{x}_{8}}^{*}\right)_{1} \leftrightarrow d \rho_{1}(\tilde{A})=-4 \varepsilon-4 \eta \leftrightarrow f_{1}^{*}\left(y_{2}, \cdots, y_{5}\right)$ : the discriminant of binary cubic forms $\leftrightarrow v_{1}+v_{7}=\left(0,0, u_{1} \wedge u_{2}+u_{3} \wedge u_{5}\right) \in S_{18,8}^{*}$

iii) $\left(S_{\tilde{x}_{8}}^{*}\right)_{2} \leftrightarrow d \rho_{2}(\tilde{A})=-6 \varepsilon-\eta \leftrightarrow f_{2}^{*}(y)=y_{1} \leftrightarrow v_{2}+v_{5}=\left(0,0, u_{1} \wedge u_{5}+u_{2}\right.$ $\left.\wedge u_{4}\right) \in S_{18,8}^{*}$

iv) $-\delta \chi=10 \varepsilon-5 \eta=-3 d \rho_{1}+2 d \rho_{2}, \operatorname{tr}_{V_{\varkappa_{8}}^{*}}=2 \varepsilon-8 \eta=2 d \rho_{1}+\frac{5}{2} d \rho_{2}$.

The conormal bundle $\Lambda_{8,18}$ is a good holonomic variety with $\operatorname{ord}_{A_{8,18}} f^{s}=$ $-5 s-\frac{8}{2}$. The conormal vector space $\left(G_{x_{8}}, \rho_{x_{8}}, V_{x_{8}}^{*}\right)$ is a regular P.V. In fact, for $z=\sum_{i=1}^{8} z_{i} v_{i} \in V_{\tilde{x}_{8}}$, the localization $f_{\tilde{x}_{8}}(z)$ of $f(x)$ is given by $f_{\tilde{x}_{8}}(z)=$ $z_{1} z_{6}^{4}+z_{1} z_{7}^{2} z_{8}^{2}+2 z_{1} z_{6}^{2} z_{7} z_{8}+z_{2}^{2} z_{8}^{3}+z_{3}^{2} z_{6}^{2} z_{8}+2 z_{2} z_{3} z_{6} z_{8}^{2}+z_{2} z_{5} z_{6}^{3}+z_{2} z_{4} z_{6}^{2} z_{8}+z_{3} z_{4} z_{6}^{3}+$ $3 z_{2} z_{5} z_{6} z_{7} z_{8}+z_{3} z_{5} z_{6}^{2} z_{7}-z_{2} z_{4} z_{7} z_{8}^{2}-z_{3} z_{4} z_{6} z_{7} z_{8}-z_{3} z_{5} z_{7}^{2} z_{8}-z_{5}^{2} z_{7}^{3}-z_{4}^{2} z_{6}^{2} z_{7}-2 z_{4} z_{5} z_{6} z_{7}^{2}$, and hence its Hessian is not identically zero. Now we shall show that $\operatorname{dim} \Lambda_{7,7}^{(1)} \cap \Lambda_{8,18}=\operatorname{dim} V-1$. From iii) above, $\left.\Lambda=\overline{G\left(\tilde{x}_{8}, v_{2}+v_{5}\right.}\right)$ is onecodimensional and $\Lambda \subset \Lambda_{8,18}$. It is enough to show $\left(\tilde{x}_{8}, v_{2}+v_{5}\right)=\left\{\left(u_{2} \wedge u_{3}\right.\right.$ $\left.\left.+u_{1} \wedge u_{4}, u_{1} \wedge u_{3}+u_{2} \wedge u_{5}, 0\right),\left(0,0, u_{1} \wedge u_{5}+u_{2} \wedge u_{4}\right)\right\} \in \Lambda_{7,7}^{(1)}$. Put $z=$ $\left\{\left(u_{1} \wedge u_{2}, u_{1} \wedge u_{3}+u_{2} \wedge u_{4}, u_{2} \wedge u_{3}+u_{1} \wedge u_{5}\right),\left(u_{1} \wedge u_{4}-u_{2} \wedge u_{5}, u_{3} \wedge u_{5}\right.\right.$, $\left.\left.u_{3} \wedge u_{4}\right)\right\}$. Then $z \in \Lambda_{7,7}^{(1)}$ (See (10)). Then for $\varepsilon>0$, put

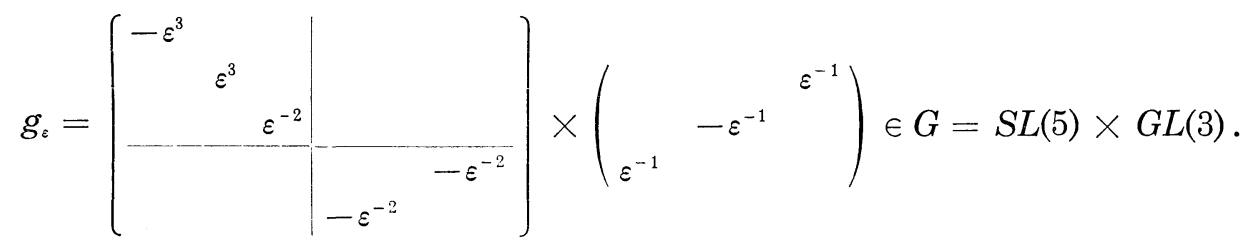


Then $g_{6} \cdot z=\left\{\left(u_{2} \wedge u_{3}+u_{1} \wedge u_{4}, u_{1} \wedge u_{3}+u_{2} \wedge u_{5},-\varepsilon^{5} u_{1} \wedge u_{2}\right),\left(-\varepsilon^{5} u_{3} \wedge u_{5}\right.\right.$, $\left.\left.\varepsilon^{5} u_{3} \wedge u_{4}, u_{1} \wedge u_{5}+u_{2} \wedge u_{4}\right)\right\}$. Since $g_{\varepsilon} \cdot z \in \Lambda_{7,7}^{(1)}$ and $\Lambda_{7,7}^{(1)}$ is closed, we have $\left(\tilde{x}_{8}\right.$, $\left.v_{2}+v_{5}\right)=\lim _{\varepsilon \rightarrow 0} g_{\varepsilon} \cdot z \in \Lambda_{7,7}^{(1)}$ and hence $\operatorname{dim} \Lambda_{7,7}^{(1)} \cap \Lambda_{8,18}=\operatorname{dim} V-1$. One can see easily that their intersection is regular and $G_{0}$-prehomogeneous. We have $b_{\Lambda_{T, 7}^{(1),}}(s) / b_{\Lambda_{8,18}}(s)=(s+1)\left(s+\frac{4}{3}\right)\left(s+\frac{5}{3}\right)$. Next we shall show that $\operatorname{dim} \Lambda_{8,18} \cap$ $\Lambda_{3,13}=\operatorname{dim} V-1$. From ii) above, $\Lambda=\overline{G\left(\tilde{x}_{8}, v_{1}+v_{7}\right)}$ is one-codimensional and $\Lambda \subset \Lambda_{8,18}$, where $\left(\tilde{x}_{8}, v_{1}+v_{7}\right)=\left\{\left(u_{2} \wedge u_{3}+u_{1} \wedge u_{4}, u_{1} \wedge u_{3}+u_{2} \wedge u_{5}, 0\right),(0,0\right.$, $\left.\left.u_{1} \wedge u_{2}+u_{3} \wedge u_{5}\right)\right\}$. Put $w=\left\{\left(u_{1} \wedge u_{2}, u_{1} \wedge u_{3}+u_{2} \wedge u_{4}, u_{2} \wedge u_{3}+u_{4} \wedge u_{5}\right)\right.$, $\left.\left(u_{2} \wedge u_{5}+u_{3} \wedge u_{4},-u_{3} \wedge u_{5}, 0\right)\right\}$. Then $w \in \Lambda_{3,13}$ (See (5)). For $\varepsilon>0$, put

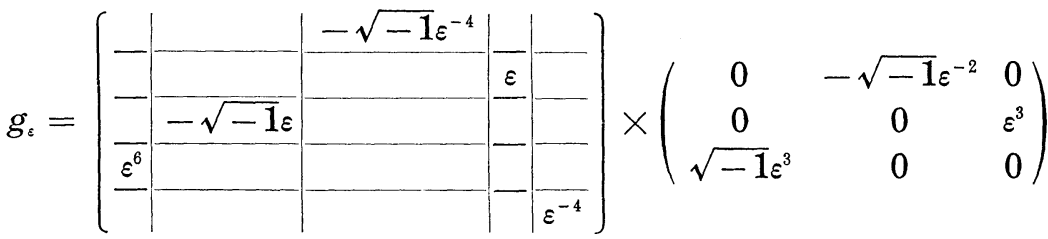

$$
\begin{aligned}
& \in G=S L(5) \times G L(3) .
\end{aligned}
$$

Then $g_{\varepsilon} \cdot w=\left\{\left(u_{2} \wedge u_{3}+u_{1} \wedge u_{4}, u_{1} \wedge u_{3}+u_{2} \wedge u_{5},-\varepsilon^{10} u_{3} \wedge u_{4}\right),\left(\varepsilon^{10} u_{3} \wedge u_{5}\right.\right.$, $\left.\left.0, u_{1} \wedge u_{2}+u_{3} \wedge u_{5}\right)\right\}$. Since $g_{c} \cdot w \in \Lambda_{3,13}$ and $\Lambda_{3,13}$ is closed, we have $\left(\tilde{x}_{8}\right.$, $\left.v_{1}+v_{7}\right)=\lim _{\imath \rightarrow 0} g_{\imath} \cdot w \in \Lambda_{3,13}$, i.e., $\Lambda \subset \Lambda_{3,13} \cap \Lambda_{8,18}$. Hence we have $\operatorname{dim} \Lambda_{3,13}$ $\cap \Lambda_{8,18}=\operatorname{dim} V-1$. The intersection is $G_{0}$-prehomogeneous and regular, and hence we have $b_{A_{3}, 18}(s) / b_{A_{3}, 13}(s)=\left(s+\frac{5}{4}\right)\left(s+\frac{7}{4}\right)$.

(13) The isotropy subalgebra $\mathfrak{g}_{x^{\prime}}$ at $x_{\dot{s}}$ is given as follows.

$$
\begin{aligned}
& \mathfrak{g}_{x^{\prime}}=\left\{\begin{array}{cc|cc|c}
\varepsilon+\alpha+\beta & \alpha_{12} & & \beta_{12} & \gamma_{1} \\
\alpha_{21} & \varepsilon-\alpha+\beta & -\beta_{12} & & \gamma_{2} \\
\hline & \beta_{21} & \varepsilon-\alpha-\beta & & \gamma_{3} \\
-\beta_{21} & & & \varepsilon+\alpha-\beta & \gamma_{4} \\
\hline & 0 & & -4 \varepsilon
\end{array}\right\} \\
& \oplus\left(\begin{array}{c|c|r}
-2 \varepsilon-2 \beta & & -\beta_{21} \\
2 \beta_{12} & -2 \varepsilon+2 \beta & \beta_{12} \\
-2 \beta_{21} & -2 \varepsilon
\end{array}\right) \cong(\mathfrak{g r}(1) \oplus \mathfrak{g r}(2) \oplus \mathfrak{g r}(2)) \oplus V(4) .
\end{aligned}
$$

Then $V_{x_{s^{\prime}}}^{*}$ is spanned by $v_{1}=\left(u_{3} \wedge u_{5}, 0,0\right), v_{2}=\left(u_{2} \wedge u_{5}, 0,-u_{3} \wedge u_{5}\right), v_{3}=$ $\left(0, u_{3} \wedge u_{5},-u_{2} \wedge u_{5}\right), v_{4}=\left(0, u_{2} \wedge u_{5}, 0\right), v_{5}=\left(u_{4} \wedge u_{5}, 0,0\right), v_{6}=\left(u_{1} \wedge u_{5}, 0\right.$, $\left.u_{4} \wedge u_{5}\right), \quad v_{7}=\left(0, u_{4} \wedge u_{5}, u_{1} \wedge u_{5}\right), \quad v_{8}=\left(0, u_{1} \wedge u_{5}, 0\right)$. We have $\left(G_{x^{\prime}}, \rho_{x^{\prime}}\right.$, $\left.V_{x_{8}^{\prime}}^{*}\right) \cong\left(G L(1) \times S L(2) \times S L(2), 5 \Lambda_{1} \otimes 3 \Lambda_{1} \otimes \Lambda_{1}, V(1) \otimes V(4) \otimes V(2)\right)$. Since $\operatorname{dim} \rho_{x_{8}^{\prime}}\left(G_{x^{\prime}}\right)=7<\operatorname{dim} V_{x_{8}^{\prime}}^{*}=8$, this is not a P.V. The dual of the orbit 
$S_{8,14}$ is $S_{14,8}^{*}$ in $V^{*}$, i.e., $\Lambda_{8,14}=\Lambda_{14,8}^{*}$.

(14) The isotropy subalgebra $\mathfrak{g}_{x_{8}^{\prime \prime}}$ at $x_{8}^{\prime \prime}$ is given as follows.

(11.14)

$$
\mathfrak{g}_{x_{\mathrm{s}}^{\prime \prime}}=\left\{\begin{array}{ccccc}
\varepsilon & \gamma_{1} & \gamma_{2} & \gamma_{3} & \gamma_{4} \\
0 & \eta & \gamma_{5} & \gamma_{6} & \gamma_{7} \\
0 & 0 & -2(\eta+\xi) & 0 & \gamma_{8} \\
0 & 0 & 0 & \xi & -\gamma_{1} \\
0 & 0 & 0 & 0 & -\varepsilon+\eta+\xi
\end{array}\right\}
$$

$$
\begin{aligned}
& \oplus\left(\begin{array}{ccc}
-(\varepsilon+\eta) & 0 & 0 \\
-\gamma_{5} & -\varepsilon+2 \eta+2 \xi & 0 \\
\gamma_{3}-\gamma_{7} & -\gamma_{8} & -\eta-\xi
\end{array}\right) \\
& \cong(\mathfrak{g l}(1) \oplus \mathfrak{g l}(1) \oplus \mathfrak{g l}(1)) \oplus \mathfrak{u}(8) .
\end{aligned}
$$

Then $V_{x_{8}^{\prime \prime}}^{*}$ is spanned by $v_{1}=\left(u_{1} \wedge u_{5}-u_{2} \wedge u_{4}, 2 u_{3} \wedge u_{4}, u_{4} \wedge u_{5}\right), v_{2}=(0$, $\left.u_{2} \wedge u_{5}, 0\right), v_{3}=\left(u_{2} \wedge u_{3}, 0, u_{3} \wedge u_{5}\right), v_{4}=\left(u_{2} \wedge u_{5},-u_{3} \wedge u_{5}, 0\right), v_{5}=\left(0, u_{4} \wedge\right.$ $\left.u_{5}, 0\right), v_{6}=\left(u_{3} \wedge u_{4}, 0,0\right), v_{7}=\left(u_{4} \wedge u_{5}, 0,0\right), v_{8}=\left(u_{3} \wedge u_{5}, 0,0\right)$.

The action $d \rho_{x_{8}^{\prime \prime}}$ of $g_{x_{8}^{\prime \prime}}$ on $V_{x_{8}^{\prime \prime}}^{*}$ is given by

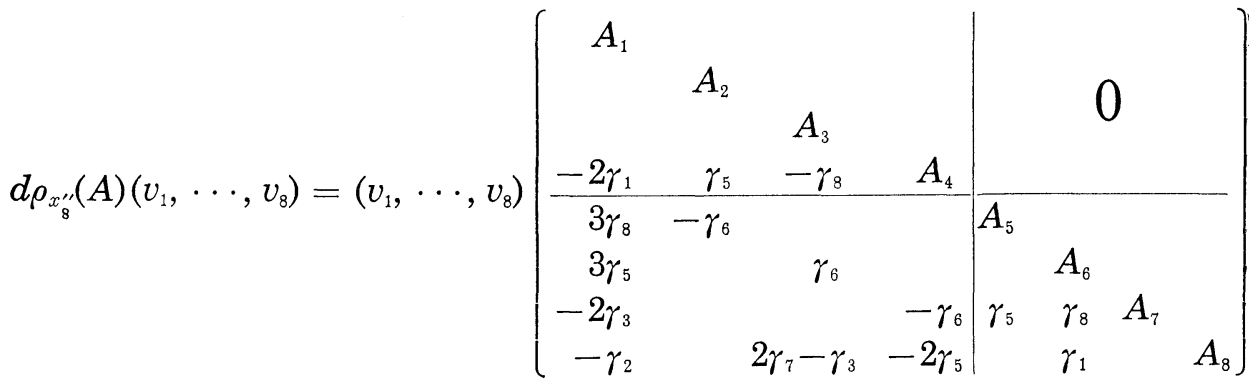

where $A_{1}=\varepsilon-\xi, A_{2}=2 \varepsilon-4 \eta-3 \xi, A_{3}=\varepsilon+2 \eta+2 \xi, A_{4}=2 \varepsilon-\eta-\xi$, $A_{5}=2 \varepsilon-3 \eta-4 \xi, A_{6}=\varepsilon+3 \eta+\xi, A_{7}=2 \varepsilon-2 \xi$ and $A_{8}=2 \varepsilon+2 \eta+\xi$.

i) $V_{x_{8^{\prime \prime}}}^{*}-S_{x^{\prime \prime}}^{*} \leftrightarrow v_{1}+v_{2}+v_{3} \in S_{5,8}^{*}$

ii) $\left(S_{x_{8}^{\prime \prime}}^{*}\right)_{1} \leftrightarrow d \rho_{1}(A)=\varepsilon-\xi \leftrightarrow f_{1}^{*}(y)=y_{1}\left(y=\sum y_{i} v_{i}\right) \leftrightarrow v_{2}+v_{3}+v_{5} \in S_{9,7}^{*}$

iii) $\left(S_{x_{8}^{\prime \prime}}^{*}\right)_{2} \leftrightarrow d \rho_{2}(A)=2 \varepsilon-4 \eta-3 \xi \leftrightarrow f_{2}^{*}(y)=y_{2} \leftrightarrow v_{1}+v_{3} \in S_{7,7}^{(2) *}$

iv) $\left(S_{x_{8}^{\prime \prime}}^{*}\right)_{3} \leftrightarrow d \rho_{3}(A)=\varepsilon+2 \eta+2 \xi \leftrightarrow f_{3}^{*}(y)=y_{3} \leftrightarrow v_{1}+v_{2} \in S_{7,7}^{(1)^{*}}$

v) $-\delta \chi=6 d \rho_{1}+d \rho_{2}+2 d \rho_{3}, \operatorname{tr}_{V x^{\prime \prime}}^{*}=\frac{15}{2} d \rho_{1}+\frac{3}{2} d \rho_{2}+\frac{5}{2} d \rho_{3}$.

Since the intersection of $\Lambda_{8,5}^{(3)}$ and $\Lambda_{7,7}^{(1)}$ is $G_{0}$-prehomogeneous, the conormal bundle $\Lambda_{8,5}^{(3)}$ is a good holonomic variety by Proposition 1-5. The order is given by $\operatorname{ord}_{\Lambda_{8,5}} f^{s}=-9 s-\frac{15}{2}$. We have $\operatorname{dim} \Lambda_{8,5}^{(3)} \cap \Lambda_{7,9}=\operatorname{dim} \Lambda_{8,5}^{(3)}$ $\cap \Lambda_{7,7}^{(i)}=\operatorname{dim} V-1(i=1,2), b_{A_{8,5}^{(3)}}(s) / b_{A_{7}^{(1), 7}}(s)=s+\frac{3}{2}$ and $b_{A_{8,5}^{(3), 5}}(s) / b_{A_{7,7}^{(2),}}(s)=$ 
$\left(s+\frac{5}{4}\right)\left(s+\frac{7}{4}\right) . \quad$ In (15), we shall prove that $\operatorname{dim} \Lambda_{8,5}^{(3)} \cap \Lambda_{7,9}^{(2)} \cap \Lambda_{5,8}^{(2)}=\operatorname{dim} \Lambda_{8,5}^{(3)}$ $\cap \Lambda_{9,7}^{(4)} \cap \Lambda_{5,8}^{(2)}=\operatorname{dim} V-1$.

(15) The isotropy subalgebra $g_{x_{9}}$ at $x_{9}$ is given as follows.

$$
\begin{aligned}
\mathfrak{g}_{x_{9}}= & \left\{A=\left(\begin{array}{ccccc}
\varepsilon_{1} & 0 & \gamma_{1} & \gamma_{2} & \gamma_{3} \\
0 & \varepsilon_{2} & \gamma_{4} & \gamma_{5} & \gamma_{6} \\
0 & 0 & \varepsilon_{3} & 0 & \gamma_{7} \\
0 & 0 & 0 & \varepsilon_{4} & \gamma_{8} \\
0 & 0 & 0 & 0 & -\left(\varepsilon_{1}+\varepsilon_{2}+\varepsilon_{3}+\varepsilon_{4}\right)
\end{array}\right)\right. \\
& \left.\oplus\left(\begin{array}{ccc}
-\varepsilon_{1}-\varepsilon_{2} & 0 & 0 \\
-\gamma_{4} & -\varepsilon_{1}-\varepsilon_{3} & 0 \\
\gamma_{2} & 0 & -\varepsilon_{2}-\varepsilon_{4}
\end{array}\right)\right\} \\
& \cong(\mathfrak{g l}(1) \oplus \mathfrak{g l}(1) \oplus \operatorname{gr}(1) \oplus \operatorname{gr}(1)) \oplus \mathfrak{u}(8) .
\end{aligned}
$$

Then $V_{x_{0}}^{*}$ is spanned by $v_{1}=\left(0, u_{2} \wedge u_{5}, 0\right), v_{2}=\left(0,0, u_{1} \wedge u_{5}\right), v_{3}=\left(u_{2} \wedge u_{5}\right.$, $\left.-u_{3} \wedge u_{5}, 0\right), v_{4}=\left(u_{1} \wedge u_{5}, 0, u_{4} \wedge u_{5}\right), v_{5}=\left(0, u_{4} \wedge u_{5}, 0\right), v_{6}=\left(0,0, u_{3} \wedge u_{5}\right)$, $v_{7}=\left(u_{3} \wedge u_{4}, 0,0\right), v_{8}=\left(u_{3} \wedge u_{5}, 0,0\right), v_{9}=\left(u_{4} \wedge u_{5}, 0,0\right)$.

The action $d \rho_{x_{9}}$ of $g_{x_{9}}$ on $V_{x_{9}}^{*}$ is given by

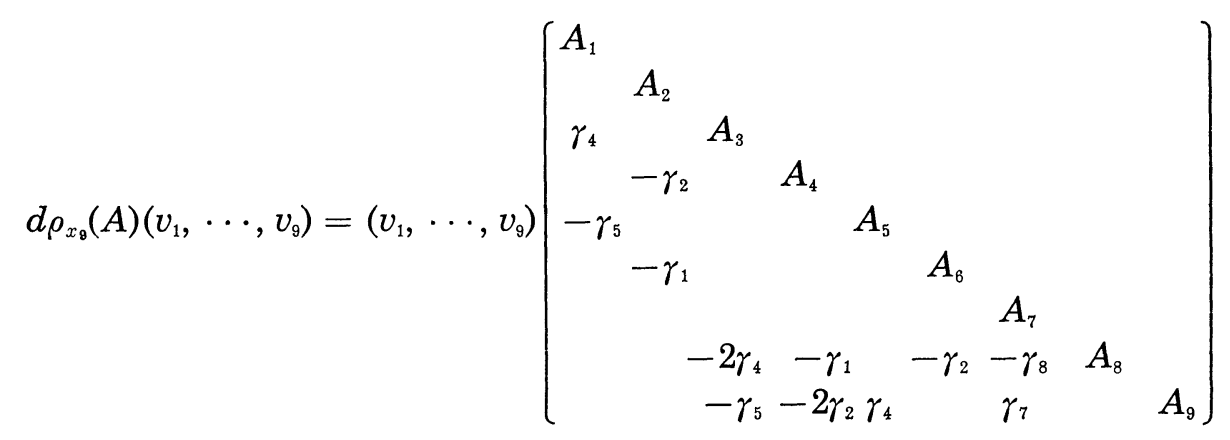

where $A_{1}=2 \varepsilon_{1}+2 \varepsilon_{3}+\varepsilon_{4}, A_{2}=2 \varepsilon_{2}+\varepsilon_{3}+2 \varepsilon_{4}, A_{3}=2 \varepsilon_{1}+\varepsilon_{2}+\varepsilon_{3}+\varepsilon_{4}, A_{4}=$ $\varepsilon_{1}+2 \varepsilon_{2}+\varepsilon_{3}+\varepsilon_{4}, A_{5}=2 \varepsilon_{1}+\varepsilon_{2}+2 \varepsilon_{3}, A_{6}=\varepsilon_{1}+2 \varepsilon_{2}+2 \varepsilon_{4}, A_{7}=\varepsilon_{1}+\varepsilon_{2}-\varepsilon_{3}-$ $\varepsilon_{4}, A_{8}=2 \varepsilon_{1}+2 \varepsilon_{2}+\varepsilon_{4}$ and $A_{9}=2 \varepsilon_{1}+2 \varepsilon_{2}+\varepsilon_{3}$.

i) $\quad V_{x_{9}}^{*}-S_{x_{9}}^{*} \leftrightarrow v_{1}+v_{2}+v_{7}=\left(u_{3} \wedge u_{4}, u_{2} \wedge u_{5}, u_{1} \wedge u_{5}\right) \in S_{7,9}^{*}$

ii) $\quad\left(S_{x_{9}}^{*}\right)_{1} \leftrightarrow d \rho_{1}(A)=2 \varepsilon_{1}+2 \varepsilon_{3}+\varepsilon_{4} \leftrightarrow f_{1}^{*}(y)=y_{1} \leftrightarrow v_{2}+v_{3}+v_{5}+v_{7} \in S_{8,5}^{*}$

iii) $\quad\left(S_{x_{9}}^{*}\right)_{2} \leftrightarrow d \rho_{2}(A)=2 \varepsilon_{2}+\varepsilon_{3}+2 \varepsilon_{4} \leftrightarrow f_{2}^{*}(y)=y_{2} \leftrightarrow v_{1}+v_{4}+v_{6}+v_{7} \in S_{8,5}^{*}$

iv) $\quad\left(S_{x_{9}}^{*}\right)_{3} \leftrightarrow d \rho_{3}(A)=\varepsilon_{1}+\varepsilon_{2}-\varepsilon_{3}-\varepsilon_{4} \leftrightarrow f_{3}^{*}(y)=y_{7} \leftrightarrow v_{1}+v_{2}+v_{8}+v_{9} \in S_{14,8}^{*}$

v) $-\delta \chi=3 d \rho_{1}+3 d \rho_{2}+4 d \rho_{3}, \operatorname{tr}_{V_{x_{9}}}^{*}=4 d \rho_{1}+4 d \rho_{2}+5 d \rho_{3}$.

The conormal bundle $\Lambda_{9,7}$ is a good holonomic variety with $\operatorname{ord}_{\Lambda_{9}, 7} f^{s}=$ $-10 s-\frac{17}{2}$. 
Put $p=\left(u_{1} \wedge u_{2}, u_{1} \wedge u_{3}, u_{2} \wedge u_{4} ; u_{1} \wedge u_{5}+u_{3} \wedge u_{4}, u_{2} \wedge u_{5}, u_{3} \wedge u_{5}+\right.$ $u_{4} \wedge u_{5}$ ). Then by iii), we have $p \in \Lambda_{9,7}^{(4)} \cap \Lambda_{5,8}^{(2)}$ and $\operatorname{dim} G \cdot p=\operatorname{dim} V-1$. We shall prove that $p \in \Lambda_{8,5}^{(3)}$, i.e., $\operatorname{dim} \Lambda_{5,8}^{(2)} \cap \Lambda_{8,5}^{(3)} \cap \Lambda_{9,7}^{(4)}=\operatorname{dim} V-1$. By (14), for any $\varepsilon>0$, we have $\left(u_{1} \wedge u_{2}, u_{1} \wedge u_{3}, u_{1} \wedge u_{5}+u_{2} \wedge u_{4} ; u_{1} \wedge u_{5}-u_{2} \wedge u_{4}\right.$ $\left.+(1 / \varepsilon) u_{2} \wedge u_{3}+\left(1 / \varepsilon^{2}\right) u_{3} \wedge u_{4}, 2 u_{3} \wedge u_{4}+u_{2} \wedge u_{5}, u_{4} \wedge u_{5}+(1 / \varepsilon) u_{3} \wedge u_{5}\right) \epsilon$

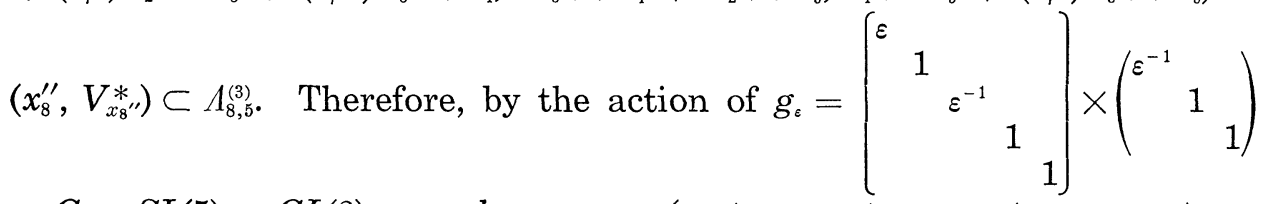
$\epsilon G=S L(5) \times G L(3)$, we have $p_{\varepsilon}=\left(u_{1} \wedge u_{2}, u_{1} \wedge u_{3}, \varepsilon u_{1} \wedge u_{5}+u_{2} \wedge u_{4}\right.$; $\left.u_{1} \wedge u_{5}-\varepsilon u_{2} \wedge u_{4}+\varepsilon u_{2} \wedge u_{3}+u_{3} \wedge u_{4}, 2 \varepsilon u_{3} \wedge u_{4}+u_{2} \wedge u_{5}, u_{3} \wedge u_{5}+u_{4} \wedge u_{5}\right)$ $\in \Lambda_{8,5}^{(3)}$. Hence, we have $p=\lim _{\varepsilon \rightarrow 0} p_{\varepsilon} \in \Lambda_{8,5}^{(3)}$. Since $\Lambda_{5,8}^{(2)}=\Lambda_{8,5}^{(3) *}, \Lambda_{8,5}^{(3)}=\Lambda_{5,8}^{(2) *}$, $\Lambda_{9,7}^{(4)}=\Lambda_{7,9}^{(2) *}$ and $(G, \rho, V) \cong\left(G, \rho^{*}, V^{*}\right)$, we have also $\operatorname{dim} \Lambda_{5,8}^{(2)} \cap \Lambda_{8,5}^{(3)} \cap \Lambda_{7,9}^{(2)}=$ $\operatorname{dim} V-1$.

(16) The isotropy subalgebra $\mathfrak{g}_{x_{10}}$ at $x_{10}$ is given as follows.

$$
\begin{aligned}
& \mathrm{g}_{x_{10}}=\left\{A=\left(\begin{array}{cc|cc|c}
\eta & \gamma_{1} & \gamma_{2} & \gamma_{3} & \gamma_{4} \\
& -4 \varepsilon & 0 & 0 & \gamma_{5} \\
\hline 0 & \varepsilon+\alpha & \alpha_{12} & \gamma_{3} \\
& \alpha_{21} & \varepsilon-\alpha & -\gamma_{2} \\
\hline 0 & \multicolumn{2}{|c|}{0} & 2 \varepsilon-\eta
\end{array}\right) \oplus\left(\begin{array}{cc|c}
4 \varepsilon-\eta & 0 & \gamma_{6} \\
-\gamma_{5} & -2 \varepsilon & \gamma_{7} \\
\hline 0 & & \\
\xi
\end{array}\right)\right\} \\
& \cong(\mathfrak{g l}(1) \oplus \mathfrak{g l}(1) \oplus \operatorname{gr}(1) \oplus \mathfrak{g l}(2)) \oplus \mathfrak{l}(7) \text {. }
\end{aligned}
$$

Then $V_{x_{10}}^{*}$ is spanned by $v_{1}=\left(0,0, u_{1} \wedge u_{3}\right), v_{2}=\left(0,0, u_{1} \wedge u_{4}\right), v_{3}=(0,0$, $\left.u_{1} \wedge u_{5}-u_{3} \wedge u_{4}\right), v_{4}=\left(0,0, u_{2} \wedge u_{3}\right), v_{5}=\left(0,0, u_{2} \wedge u_{4}\right), v_{6}=\left(u_{3} \wedge u_{5}, 0,0\right)$, $v_{7}=\left(u_{4} \wedge u_{5}, 0,0\right), v_{8}=\left(0,0, u_{3} \wedge u_{5}\right), v_{9}=\left(0,0, u_{4} \wedge u_{5}\right), v_{10}=\left(0,0, u_{2} \wedge u_{5}\right)$.

$$
V_{x_{10}}^{*}-S_{x_{10}}^{*} \leftrightarrow v_{1}+v_{5}+v_{7}=\left(u_{4} \wedge u_{5}, 0, u_{1} \wedge u_{3}+u_{2} \wedge u_{4}\right)=y_{10} .
$$

Let $A_{0}$ be an element of $g_{x_{10}}$ with $\alpha=-\frac{1}{3}-5 \varepsilon, \eta=\frac{2}{3}+6 \varepsilon, \xi=-\frac{4}{3}-2 \varepsilon$, all remaining parts zero in (5.16). Then $d \rho\left(A_{0}\right) x_{10}=0$ and $d \rho^{*}\left(A_{0}\right) y_{10}=y_{10}$. Since $-\delta \chi\left(A_{0}\right)=10(1+3 \varepsilon)$ is not definite, the conormal bundle $\Lambda_{10,10}$ is not a good holonomic variety.

(17) The isotropy subalgebra $\mathfrak{g}_{x_{11}}$ at $x_{11}$ is given as follows.

$$
\begin{aligned}
& \mathrm{g}_{x_{11}}=\left\{A=\left(\begin{array}{c|cc|c|c}
\varepsilon+\eta & \gamma_{1} & \gamma_{2} & \gamma_{3} & \gamma_{4} \\
\hline 0 & \varepsilon+\alpha & \alpha_{12} & \gamma_{5} & \gamma_{6} \\
\hline 0 & \alpha_{21} & \varepsilon-\alpha & \gamma_{7} & \gamma_{8} \\
\hline \varepsilon & 0 & \begin{array}{c}
\text { (11.17) } \\
n_{0}
\end{array} & \gamma_{9}
\end{array}\right) \oplus\left(\begin{array}{c|c|c}
-2 \varepsilon-\eta-\alpha & -\alpha_{21} & 0 \\
-\alpha_{12} & -2 \varepsilon-\eta+\alpha & \\
\hline \gamma_{2}-\gamma_{5} & -\gamma_{1}-\gamma_{7} & -2 \varepsilon
\end{array}\right)\right\} \\
& \cong(\mathfrak{g} l(1) \oplus \mathfrak{g} \mathfrak{l}(1) \oplus \mathfrak{g} \mathfrak{l}(2)) \oplus \mathfrak{u}(9) \text {. }
\end{aligned}
$$


The conormal vector space $V_{x_{11}}^{*}$ is spanned by $v_{1}=\left(0, u_{1} \wedge u_{5},-u_{2} \wedge u_{5}\right)$, $v_{2}=\left(u_{1} \wedge u_{5}, 0, u_{3} \wedge u_{5}\right), v_{3}=\left(u_{2} \wedge u_{5}, 0,-u_{4} \wedge u_{5}\right), v_{4}=\left(u_{2} \wedge u_{4},-u_{3} \wedge u_{4}, 0\right)$, $v_{5}=\left(0, u_{2} \wedge u_{4}, 0\right), \quad v_{6}=\left(u_{3} \wedge u_{4}, 0,0\right), \quad v_{7}=\left(u_{2} \wedge u_{5},-u_{3} \wedge u_{5}, 0\right), \quad v_{8}=(0$, $\left.u_{2} \wedge u_{5}, 0\right), v_{9}=\left(u_{3} \wedge u_{5}, 0,0\right), v_{10}=\left(0, u_{4} \wedge u_{5}, 0\right), v_{11}=\left(u_{4} \wedge u_{5}, 0,0\right)$.

The action $d \rho_{x_{11}}$ of $g_{x_{11}}$ on $V_{x_{11}}^{*}$ is given by

$$
d \rho_{x_{11}}(A)\left(v_{1}, \cdots, v_{11}\right)\left(\begin{array}{lllll}
A_{1} & & & & \\
B_{1} & A_{2} & & & \\
& & A_{3} & & \\
B_{2} & A_{6} & B_{3} & A_{4} & \\
\underbrace{B_{4}}_{2} & \underbrace{B_{5}}_{1} & \underbrace{B_{6}}_{3} & \underbrace{B_{7}}_{3} & \underbrace{A_{5}}_{2}\} \\
\}_{3} \\
\}_{3} \\
\}_{2}
\end{array}\right.
$$

where $\left(B_{1}, A_{2}\right)=\left(-\gamma_{5}, \gamma_{7}, 5 \varepsilon+\eta\right), B_{3}=-\gamma_{9} \cdot I_{3}, B_{4}=-\gamma_{3} \cdot I_{2}, A_{1}=5 \varepsilon I_{2}+A^{\prime}$, $A_{5}=(5 \varepsilon+2 \eta) I_{2}+A^{\prime}$ with $A^{\prime}=\left(\begin{array}{cc}-\alpha & \alpha_{21} \\ \alpha_{12} & \alpha\end{array}\right)$,

$$
\begin{gathered}
\left(B_{5}, B_{6}, B_{7}\right)=\left(\begin{array}{c|ccc|ccc}
-\gamma_{1}-\gamma_{7} & -\gamma_{8} & \gamma_{6} & 0 & -\gamma_{7} & -\gamma_{5} & 0 \\
\gamma_{2}-2 \gamma_{5} & \gamma_{6} & 0 & \gamma_{8} & -\gamma_{5} & 0 & -\gamma_{7}
\end{array}\right), \\
A_{3}=2 \eta \cdot I_{3}+A^{\prime \prime}, A_{4}=(5 \varepsilon+\eta) I_{3}+A^{\prime \prime} \\
\text { with } A^{\prime \prime}=\left(\begin{array}{ccc}
0 & \alpha_{12} & -\alpha_{21} \\
2 \alpha_{21} & -2 \alpha & 0 \\
-2 \alpha_{12} & 0 & 2 \alpha
\end{array}\right), \\
\left(B_{2}, A_{6}\right)=\left(\begin{array}{ccc}
-\gamma_{2} & -\gamma_{1}-\gamma_{7} & \\
-2 \gamma_{1}-\gamma_{7} & & \alpha_{21} \\
0 & \gamma_{5}-2 \gamma_{2} & -\alpha_{12}
\end{array}\right) .
\end{gathered}
$$

Note that $A_{6}$ will disappear if we take $v_{3}-\frac{1}{2} v_{7}$ instead of $v_{3}$.

i) $V_{x_{11}}^{*}-S_{x_{11}}^{*} \leftrightarrow v_{1}+v_{2}+v_{4}=\left(u_{1} \wedge u_{5}+u_{2} \wedge u_{4}, u_{1} \wedge u_{5}-u_{3} \wedge u_{4}, u_{3} \wedge u_{5}\right.$ $\left.-u_{2} \wedge u_{5}\right) \in S_{4,11}^{(3) *}$

ii) $\left(S_{x_{11}}^{*}\right)_{1} \leftrightarrow v_{1}+v_{4}+v_{9}=\left(u_{2} \wedge u_{4}+u_{3} \wedge u_{5}, u_{1} \wedge u_{5}-u_{3} \wedge u_{4},-u_{2} \wedge u_{5}\right)$ $\in S_{5,8}^{(2) *} \leftrightarrow d \rho_{1}=10 \varepsilon+2 \eta$

iii) $\left(S_{x_{11}}^{*}\right)_{2} \leftrightarrow v_{2}+v_{5}=\left(u_{1} \wedge u_{5}, u_{2} \wedge u_{4}, u_{3} \wedge u_{5}\right) \in S_{7,9}^{(2) *} \leftrightarrow d \rho_{2}=4 \eta \leftrightarrow f_{2}^{*}(y)$ $=y_{4}^{2}+y_{5} y_{6}$

iv) $-\delta \chi=30 \varepsilon+10 \eta=3 d \rho_{1}+d \rho_{2}, \operatorname{tr}_{V_{x_{11}}^{*}}=40 \varepsilon+14 \eta=4 d \rho_{1}+\frac{3}{2} d \rho_{2}$.

Remark for calculation of $d \rho_{1}$. Let $f_{1}^{*}$ be the relative invariant on $V_{x_{11}}^{*}$ corresponding to $d \rho_{1}$. Since $f_{1}^{*}\left(v_{2}+v_{5}\right) \neq 0$, the restriction of $d \rho_{1}$ to the isotropy subalgebra of $g_{x_{11}}$ at $v_{2}+v_{5}$ should be zero. Hence $d \rho_{1}$ must be of the form $d \rho_{1}=5 \lambda \varepsilon+\lambda \eta$ for some $\lambda$. Take an element $A_{0}$ in $\mathrm{g}_{x_{11}}$ satisfying $d \rho^{*}\left(A_{0}\right) x_{11}^{*}=x_{11}^{*}$ where $x_{11}^{*}=v_{1}+v_{2}+v_{4} \in V_{x_{11}}^{*}-S_{x_{11}}^{*}$. Then, by the Euler's identity, we have $\left(\operatorname{deg} f_{1}^{*}\right) \cdot f_{1}^{*}\left(x_{11}^{*}\right)=\left.\left\langle d \rho^{*}\left(A_{0}\right) y, D_{y}\right\rangle f_{1}^{*}(y)\right|_{y=x_{11}^{*}}=$ 
$d \rho_{1}\left(A_{0}\right) f_{1}^{*}\left(x_{11}\right)$ and hence $\operatorname{deg} f_{1}^{*}=d \rho_{1}\left(A_{0}\right)=\frac{3}{2} \lambda \in N$. Therefore $d \rho_{1}=(10 \varepsilon$ $+2 \eta) \mu$ where $\mu$ is a natural number. Since $-\delta \chi$ is a linear combination of $d \rho_{1}$ and $d \rho_{2}$ with coefficients in $Z$, we have $\mu=1$ or 3 . On the other hand, $2 \operatorname{tr}_{V_{x_{11}^{*}}^{*}}$ is also a linear combination of $d \rho_{1}$ and $d \rho_{2}$ with coefficients in $Z, \mu$ is a divisor of 8 , and hence $\mu=1$, i.e., $d \rho_{1}=10 \varepsilon+2 \eta$.

(18) The isotropy subalgebra $g_{x_{12}}$ at $x_{12}$ is given as follows.

$$
\begin{aligned}
\mathfrak{g}_{x_{12}}= & \left\{A=\left(\begin{array}{cc|c|c|c}
\varepsilon+\alpha & \alpha_{12} & 0 & \gamma_{1} \\
\alpha_{21} & \varepsilon-\alpha & 0 & \gamma_{2} \\
\hline 0 & \eta+\beta & \beta_{12} & \gamma_{3} \\
\beta_{21} & \eta-\beta & \gamma_{4} \\
\hline 0 & 0 & -2(\varepsilon+\eta)
\end{array}\right) \oplus\left(\begin{array}{cc|c}
-2 \varepsilon & 0 & \gamma_{5} \\
0 & -2 \eta & \gamma_{6} \\
\hline & 0 & \xi
\end{array}\right)\right\} \\
& \cong(\mathfrak{g l}(1) \oplus \mathfrak{g l}(1) \oplus \mathfrak{g l}(1) \oplus \mathfrak{g l}(2) \oplus \mathfrak{g l}(2)) \oplus V(6) .
\end{aligned}
$$

The conormal vector space $V_{x_{12}}^{*}$ is spanned by $v_{1}=\left(0,0, u_{1} \wedge u_{5}\right), v_{2}=(0,0$, $\left.u_{2} \wedge u_{5}\right), v_{3}=\left(0,0, u_{3} \wedge u_{5}\right), v_{4}=\left(0,0, u_{4} \wedge u_{5}\right), v_{5}=\left(0, u_{1} \wedge u_{5}, 0\right), v_{6}=(0$, $\left.u_{2} \wedge u_{5}, 0\right), v_{7}=\left(u_{3} \wedge u_{5}, 0,0\right), v_{8}=\left(u_{4} \wedge u_{5}, 0,0\right), v_{9}=\left(0,0, u_{1} \wedge u_{3}\right), v_{10}=$ $\left(0,0, u_{1} \wedge u_{4}\right), v_{11}=\left(0,0, u_{2} \wedge u_{3}\right), v_{12}=\left(0,0, u_{2} \wedge u_{4}\right)$.

The action $d \rho_{x_{12}}$ of $\mathfrak{g}_{x_{12}}$ on $V_{x_{12}}^{*}$ is given by

$$
\left.d \rho_{x_{12}}(A)\left(v_{1}, \cdots, v_{12}\right)=\left(v_{1}, \cdots, v_{12}\right)=\left(\begin{array}{llllll}
A_{1} & & B_{1} & & B_{2} \\
& A_{2} & & B_{3} & B_{4} \\
& & A_{3} & & \\
& & & A_{4} & \\
& & \underbrace{}_{2} & \underbrace{}_{2} & \underbrace{A_{5}}_{4}
\end{array}\right\}_{2}\right\}_{2}
$$

where $B_{1}=-\gamma_{6} I_{2}, B_{3}=-\gamma_{5} I_{2}, A_{1}=(\varepsilon+2 \eta-\xi) I_{2}+A^{\prime}, A_{2}=(2 \varepsilon+\eta-\xi) I_{2}$ $+B^{\prime}, \quad A_{3}=(\varepsilon+4 \eta) I_{2}+A^{\prime}, \quad A_{4}=(4 \varepsilon+\eta) I_{2}+B^{\prime}$ with $A^{\prime}=\left(\begin{array}{cc}-\alpha & -\alpha_{21} \\ -\alpha_{12} & \alpha\end{array}\right)$, $B^{\prime}=\left(\begin{array}{cc}-\beta & -\beta_{21} \\ -\beta_{12} & \beta\end{array}\right), \quad A_{5}=-(\varepsilon+\eta+\xi) I_{4}+\left(\begin{array}{ll}-\alpha I_{2}+B^{\prime} & -\alpha_{21} I_{2} \\ -\alpha_{12} I_{2} & \alpha I_{2}+B^{\prime}\end{array}\right), \quad B_{2}=$ $\left(\begin{array}{cccc}-\gamma_{3} & -\gamma_{4} & 0 & 0 \\ 0 & 0 & -\gamma_{3} & -\gamma_{4}\end{array}\right)$ and $B_{4}=\left(\begin{array}{llll}\gamma_{1} & 0 & \gamma_{2} & 0 \\ 0 & \gamma_{1} & 0 & \gamma_{2}\end{array}\right)$.

i) $V_{x_{12}}^{*}-S_{x_{12}}^{*} \leftrightarrow v_{6}+v_{7}+v_{10}+v_{11}=\left(u_{3} \wedge u_{5}, u_{2} \wedge u_{5}, u_{1} \wedge u_{4}+u_{2} \wedge u_{3}\right)$ $\in S_{6,12}^{(1) *}$

ii) $\left(S_{x_{12}}^{*}\right)_{1} \leftrightarrow v_{6}+v_{8}+v_{9}=\left(u_{4} \wedge u_{5}, u_{2} \wedge u_{5}, u_{1} \wedge u_{3}\right) \in S_{7,9}^{(2) *} \leftrightarrow d \rho_{1}$

$$
=-2(\varepsilon+\eta+\xi) \leftrightarrow f_{1}^{*}(y)=y_{10} y_{11}-y_{9} y_{12}
$$

iii) $\left(S_{x_{12}}^{*}\right)_{2} \leftrightarrow v_{5}+v_{7}+v_{10}+v_{11}=\left(u_{3} \wedge u_{5}, u_{1} \wedge u_{5}, u_{2} \wedge u_{3}+u_{2} \wedge u_{4}\right)$

$$
\in S_{7,7}^{(2) *} \leftrightarrow d \rho_{2}=4(\varepsilon+\eta)-\xi \leftrightarrow \operatorname{deg} f_{2}^{*}(y)=3
$$

iv) $-\delta \chi=10(\varepsilon+\eta)-5 \xi=d \rho_{1}+3 d \rho_{2}, \operatorname{tr}_{V x_{12}}^{*}=12(\varepsilon+\eta)-8 \xi=2 d \rho_{1}+4 d \rho_{2}$.

(19) The isotropy subalgebra $g_{x_{13}}$ at $x_{13}$ is given as follows. 


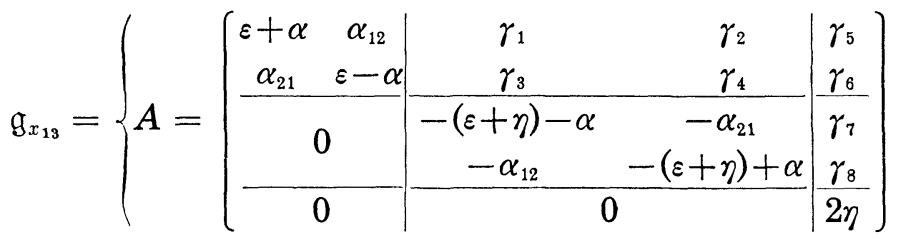

$$
\begin{aligned}
& \left.\oplus\left(\begin{array}{ccc}
-2 \varepsilon & 0 & \delta_{1} \\
\gamma_{2}-\gamma_{3} & \eta & \delta_{2} \\
0 & 0 & \xi
\end{array}\right)\right\}(\mathfrak{g l}(1) \oplus \mathfrak{g} \mathfrak{l}(1) \oplus \mathfrak{g} \mathfrak{l}(1) \oplus \mathfrak{g l}(2)) \oplus \mathfrak{u}(10) .
\end{aligned}
$$

The conormal vector space $V_{x_{13}}^{*}$ is spanned by $v_{1}=\left(0,0,-u_{4} \wedge u_{5}\right), v_{2}=$ $\left(0,0, u_{3} \wedge u_{5}\right), v_{3}=\left(0,0, u_{1} \wedge u_{5}\right), v_{4}=\left(0,0, u_{2} \wedge u_{5}\right), v_{5}=\left(0,0, u_{3} \wedge u_{4}\right), v_{6}$ $=\left(0,0,-u_{1} \wedge u_{4}\right), \quad v_{7}=\left(0,0, u_{1} \wedge u_{3}-u_{2} \wedge u_{4}\right), \quad v_{8}=\left(0,0, u_{2} \wedge u_{3}\right), \quad v_{9}=$ $\left(-u_{4} \wedge u_{5}, 0,0\right), v_{10}=\left(u_{3} \wedge u_{5}, 0,0\right), v_{11}=\left(u_{3} \wedge u_{4}, 0,0\right), v_{12}=\left(u_{1} \wedge u_{5}, u_{4} \wedge\right.$ $\left.u_{5}, 0\right), v_{13}=\left(u_{2} \wedge u_{5},-u_{3} \wedge u_{5}, 0\right)$.

The action $d \rho_{x_{13}}$ of $g_{x_{13}}$ on $V_{x_{13}}^{*}$ is given by

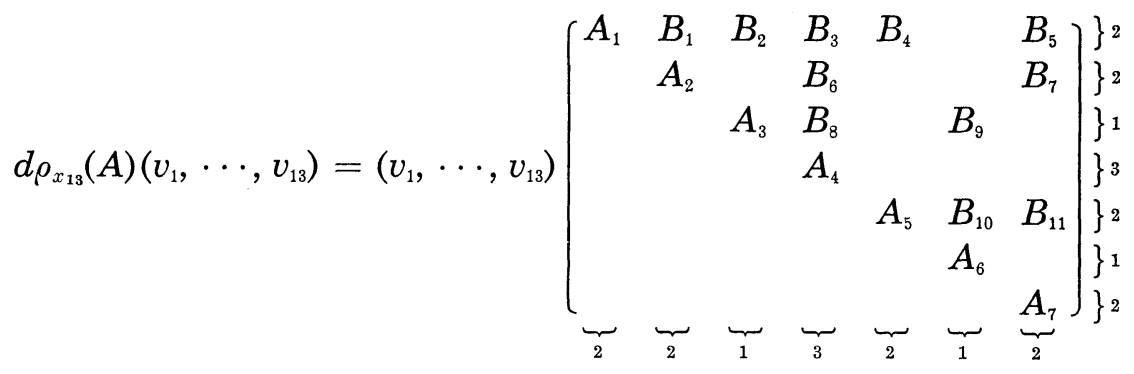

where $A_{3}=2 \varepsilon+2 \eta-\xi, B_{8}=\left(\gamma_{1}, \gamma_{2}+\gamma_{3}, \gamma_{4}\right), B_{9}=-\delta_{1}, A_{6}=4 \varepsilon+2 \eta, B_{4}=$ $B_{7}=-\delta_{1} I_{2}, \quad B_{5}=\delta_{2} I_{2}, \quad A_{1}=(\varepsilon-\eta-\xi) I_{2}+A^{\prime} \quad$ with $\quad A^{\prime}=\left(\begin{array}{cc}-\alpha & -\alpha_{21} \\ -\alpha_{12} & \alpha\end{array}\right)$, $\left(B_{1}, B_{2}, B_{3}\right)=\left(\begin{array}{rrrrrr}\gamma_{2} & \gamma_{4} & -\gamma_{7} & \gamma_{5} & \gamma_{6} & 0 \\ -\gamma_{1} & -\gamma_{3} & -\gamma_{8} & 0 & \gamma_{5} & \gamma_{6}\end{array}\right), B_{6}=\left(\begin{array}{rrr}\gamma_{8} & -\gamma_{7} & \\ & \gamma_{8} & -\gamma_{7}\end{array}\right),\left(B_{10}, B_{11}\right)=$ $\left(\begin{array}{ccc}-\gamma_{7} & 2 \gamma_{2}-\gamma_{3} & \gamma_{4} \\ -\gamma_{8} & -\gamma_{1} & \gamma_{2}-2 \gamma_{3}\end{array}\right), A_{2}=-(\varepsilon+2 \eta+\xi) I_{2}+A^{\prime}, A_{5}=(3 \varepsilon-\eta) I_{2}+A^{\prime}, A_{7}$ $=(\varepsilon-2 \eta) I_{2}+A^{\prime}$ and $A_{4}=(\eta-\xi) I_{3}+\left(\begin{array}{ccc}-2 \alpha & -2 \alpha_{21} & 0 \\ -\alpha_{12} & 0 & -\alpha_{21} \\ 0 & -2 \alpha_{12} & 2 \alpha\end{array}\right)$.

i) $V_{x_{13}}^{*}-S_{x_{13}}^{*} \leftrightarrow v_{7}+v_{11}+v_{12}+v_{13} \in S_{3,13}^{(2) *}$

ii) $\left(S_{x_{13}}^{*}\right)_{1} \leftrightarrow v_{7}+v_{12}+v_{13} \in S_{6,12}^{(1) *} \leftrightarrow d \rho_{1}=4 \varepsilon+2 \eta \leftrightarrow f_{1}^{*}(y)=y_{11}$

iii) $\left(S_{x_{13}}^{*}\right)_{2} \leftrightarrow v_{8}+v_{11}+v_{12} \in S_{4,11}^{(3) *} \leftrightarrow d \rho_{2}=2(\eta-\xi) \leftrightarrow f_{2}^{*}(y)=y_{7}^{2}-y_{6} y_{8}$

iv) $\left(S_{x_{13}}^{*}\right)_{3} \leftrightarrow v_{7}+v_{11}+v_{12} \in S_{5,8}^{(2) *} \leftrightarrow d \rho_{3}=2 \varepsilon-3 \eta-\xi \leftrightarrow \operatorname{deg} f_{2}^{*}(y)=3$

v) $-\delta \chi=d \rho_{1}+d \rho_{2}+3 d \rho_{3}, \operatorname{tr}_{V x_{13}}^{*}=\frac{3}{2} d \rho_{1}+2 d \rho_{2}+4 d \rho_{3}$.

(20) The isotropy subalgebra $\mathfrak{g}_{x_{14}}$ at $x_{14}$ is given as follows. 


$$
\begin{aligned}
& \mathfrak{g}_{x_{14}}=\left\{A=\left(\begin{array}{c|c|c}
\frac{\varepsilon}{0} & \frac{*}{\eta I_{3}+X} & \frac{*}{*} \\
\hline 0 & \frac{*}{-\varepsilon-3 \eta}
\end{array}\right) \oplus\left(-(\varepsilon+\eta) I_{3}-{ }^{t} X\right) ; X \in \mathfrak{g r}(3)\right\} \\
& \cong(\mathfrak{g l}(1) \oplus \mathfrak{g} \mathfrak{l}(1) \oplus \mathfrak{g} \mathfrak{l}(3)) \oplus \mathfrak{u}(7) \text {. }
\end{aligned}
$$

The conormal vector space $V_{x_{14}}^{*}$ is spanned by $v_{1}=\left(u_{3} \wedge u_{4}, 0,0\right), v_{2}=(0$, $\left.u_{2} \wedge u_{4}, 0\right), v_{3}=\left(0,0, u_{2} \wedge u_{3}\right), v_{4}=\left(0, u_{2} \wedge u_{3},-u_{2} \wedge u_{4}\right), v_{5}=\left(u_{2} \wedge u_{3}, 0\right.$, $\left.u_{3} \wedge u_{4}\right), \quad v_{6}=\left(u_{2} \wedge u_{4},-u_{3} \wedge u_{4}, 0\right), \quad v_{7}=\left(u_{2} \wedge u_{5},-u_{3} \wedge u_{5}, 0\right), \quad v_{8}=(0$, $\left.u_{3} \wedge u_{5},-u_{4} \wedge u_{5}\right), v_{9}=\left(0, u_{2} \wedge u_{5}, 0\right), v_{10}=\left(0,0, u_{3} \wedge u_{5}\right), v_{11}=\left(u_{4} \wedge u_{5}, 0,0\right)$, $v_{12}=\left(u_{3} \wedge u_{5}, 0,0\right), v_{13}=\left(0, u_{4} \wedge u_{5}, 0\right), v_{14}=\left(0,0, u_{2} \wedge u_{5}\right)$.

Since $\operatorname{dim} \rho_{x_{14}}\left(G_{x_{14}}\right)=13$ and $\operatorname{dim} V_{x_{14}}^{*}=14$, the conormal vector space $\left(G_{x_{14}}, \rho_{x_{14}}, V_{x_{14}}^{*}\right)$ is not a P.V. Note that it is also obtained from the fact that $\Lambda_{8,14}=\Lambda_{14,8}^{*}$ is not $G$-prehomogeneous (See (13)).

(21) The isotropy subalgebra $\mathfrak{g}_{x_{15}}$ at $x_{15}$ is given as follows.

$$
\begin{aligned}
& \mathfrak{g}_{x_{15}}=\left\{A=\left(\frac{2 \varepsilon I_{3}+X}{0} \mid \frac{Z}{-3 \varepsilon I_{2}+Y}\right) \oplus\left(-4 \varepsilon I_{3}+S^{-1} X S\right) ; X \in \mathfrak{g l}(3)\right. \\
&\left.Y \in \mathfrak{g l}(2), Z \in V(6), S=\left(\begin{array}{c}
1 \\
-1 \\
1
\end{array}\right)\right\} \\
& \cong(\mathfrak{g l}(1) \oplus \mathfrak{g l}(3) \oplus \mathfrak{g l}(2)) \oplus V(6) .
\end{aligned}
$$

The conormal vector space $V_{x_{15}}^{*}$ is spanned by $v_{1}=\left(0,0, u_{1} \wedge u_{4}\right), v_{2}=(0$, $\left.u_{2} \wedge u_{4}, 0\right), \quad v_{3}=\left(u_{3} \wedge u_{4}, 0,0\right), \quad v_{4}=\left(0, u_{1} \wedge u_{4},-u_{2} \wedge u_{4}\right), \quad v_{5}=\left(u_{2} \wedge u_{4}\right.$, $\left.-u_{3} \wedge u_{4}, 0\right), v_{6}=\left(u_{1} \wedge u_{4}, 0, u_{3} \wedge u_{4}\right), v_{7}=\left(0,0, u_{1} \wedge u_{5}\right), v_{8}=\left(0, u_{2} \wedge u_{5}, 0\right)$, $v_{9}=\left(u_{3} \wedge u_{5}, 0,0\right), v_{10}=\left(0, u_{1} \wedge u_{5},-u_{2} \wedge u_{5}\right), v_{11}=\left(u_{2} \wedge u_{5},-u_{3} \wedge u_{5}, 0\right)$, $v_{12}=\left(u_{1} \wedge u_{5}, 0, u_{3} \wedge u_{5}\right), v_{13}=\left(0,0, u_{4} \wedge u_{5}\right), v_{14}=\left(0, u_{4} \wedge u_{5}, 0\right), v_{15}=\left(u_{4} \wedge\right.$ $\left.u_{5}, 0,0\right)$. Then the action $d \rho_{x_{15}}$ of $\mathfrak{g}_{x_{15}}$ on $V_{x_{15}}^{*}$ is given by

$$
\left(v_{1}, \cdots, v_{15}\right) \mapsto\left(v_{1}, \cdots, v_{15}\right)\left(\begin{array}{c|c}
G L(1) \times S L(3) \times S L(2) & 0 \\
5 \Lambda_{1} \otimes 2 \Lambda_{1}^{*} \otimes \Lambda_{1}^{*} & \frac{1}{G L(1) \times S L(3)} \\
\hline * & 10 \Lambda_{1} \otimes \Lambda_{1}^{*}
\end{array}\right)
$$

$$
\begin{gathered}
\text { i) } V_{x_{15}}^{*}-S_{x_{15}}^{*} \leftrightarrow v_{1}+v_{2}+v_{7}+v_{9}=\left(u_{3} \wedge u_{5}, u_{2} \wedge u_{4}, u_{1} \wedge u_{4}+u_{1} \wedge u_{5}\right) \\
\in S_{3,15}^{(3) *} \\
\text { ii) } \quad\left(S_{x_{15}}^{*}\right)_{1} \leftrightarrow v_{4}+v_{11}+v_{12} \in S_{4,11}^{(3) *} \leftrightarrow d \rho_{1}=60 \varepsilon \\
\text { iii) } \quad-\delta \chi=60 \varepsilon=d \rho_{1}, \operatorname{tr}_{V_{x_{15}}^{*}}=90 \varepsilon=\frac{3}{2} d \rho_{1} .
\end{gathered}
$$

(22) The isotropy subalgebra $g_{x_{16}}$ at $x_{16}$ is given as follows. 


$$
\begin{aligned}
& \mathfrak{g}_{x_{16}}=\left\{A=\left(\begin{array}{c|cc|cc}
-2(\varepsilon+\eta) & \gamma_{1} & \gamma_{2} & \gamma_{3} & \gamma_{4} \\
\hline 0 & \varepsilon+\alpha_{1} & \alpha_{12} & \gamma_{5} & \gamma_{6} \\
\alpha_{21} & \varepsilon-\alpha_{1} & \gamma_{7} & \gamma_{8} \\
\hline 0 & 0 & \begin{array}{c}
\eta+\beta_{1} \\
\beta_{21}
\end{array} & \beta_{12} \\
\hline 0-\beta_{1}
\end{array}\right\}\right. \\
& \left.\oplus\left(\begin{array}{cc|c}
\varepsilon+2 \eta-\alpha_{1} & -\alpha_{21} & \gamma_{9} \\
-\alpha_{12} & \varepsilon+2 \eta+\alpha_{1} & \gamma_{10} \\
\hline \xi
\end{array}\right)\right\} \\
& \cong(\mathfrak{g l}(1) \oplus \mathfrak{g l}(1) \oplus \mathfrak{g l}(1) \oplus \mathfrak{g l}(2) \oplus \mathfrak{g l}(2)) \oplus \mathfrak{H}(10) \text {. }
\end{aligned}
$$

The conormal vector space $V_{x_{16}}^{*}$ is spanned by $v_{1}=\left(0,0, u_{4} \wedge u_{5}\right), v_{2}=(0$, $\left.-u_{4} \wedge u_{5}, 0\right), v_{3}=\left(u_{4} \wedge u_{5}, 0,0\right), v_{4}=\left(0,0, u_{2} \wedge u_{4}\right), v_{5}=\left(0,0, u_{2} \wedge u_{5}\right), v_{6}$ $=\left(0,0, u_{3} \wedge u_{4}\right), v_{7}=\left(0,0, u_{3} \wedge u_{5}\right), \quad v_{8}=\left(0,0, u_{2} \wedge u_{3}\right), v_{9}=\left(0,0, u_{1} \wedge u_{4}\right)$, $v_{10}=\left(0,0, u_{1} \wedge u_{5}\right), v_{11}=\left(0,-u_{2} \wedge u_{4}, 0\right), v_{12}=\left(u_{2} \wedge u_{4},-u_{3} \wedge u_{4}, 0\right), v_{13}=$ $\left(u_{3} \wedge u_{4}, 0,0\right), v_{14}=\left(0,-u_{2} \wedge u_{5}, 0\right), v_{15}=\left(u_{2} \wedge u_{5},-u_{3} \wedge u_{5}, 0\right), v_{16}=\left(u_{3} \wedge\right.$ $\left.u_{5}, 0,0\right)$.

The action $d \rho_{x_{16}}$ of $g_{x_{16}}$ on $V_{x_{16}}^{*}$ is given by

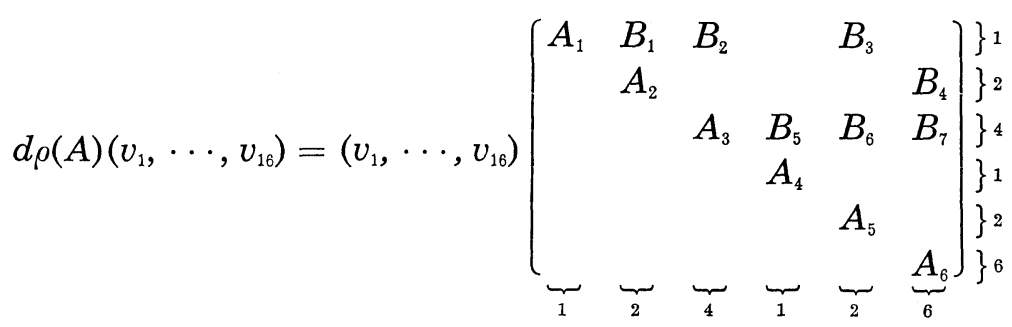

where $\left(A_{1}, B_{1}, B_{2}\right)=\left(-2 \eta-\xi, \gamma_{10},-\gamma_{9}, \gamma_{6},-\gamma_{5}, \gamma_{8},-\gamma_{7}\right), B_{3}=\left(\gamma_{4},-\gamma_{3}\right), A_{4}$

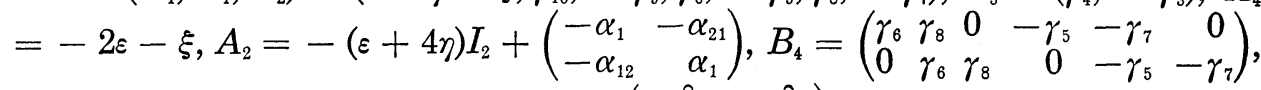
$A_{5}=(2 \varepsilon+\eta-\xi) I_{2}+B$ with $B=\left(\begin{array}{cc}-\beta_{1} & -\beta_{21} \\ -\beta_{12} & \beta_{1}\end{array}\right), \quad A_{3}=-(\varepsilon+\eta+\xi) I_{4}+$ $\left(\frac{-\alpha_{1} I_{2}+B}{-\alpha_{12} I_{2}} \mid \frac{-\alpha_{21} I_{2}}{\alpha_{1} I_{2}+B}\right), A_{6}=-(2 \varepsilon+3 \eta) I_{6}+\left(\frac{-\beta_{1} I_{3}+A^{\prime}}{-\beta_{12} I_{3}} \mid \frac{-\beta_{21} I_{3}}{\beta_{1} I_{3}+A^{\prime}}\right)$ with $A^{\prime}$ $=\left(\begin{array}{ccc}-2 \alpha_{1} & -2 \alpha_{21} & 0 \\ -\alpha_{12} & 0 & -\alpha_{21} \\ 0 & -2 \alpha_{12} & 2 \alpha_{1}\end{array}\right)$ and

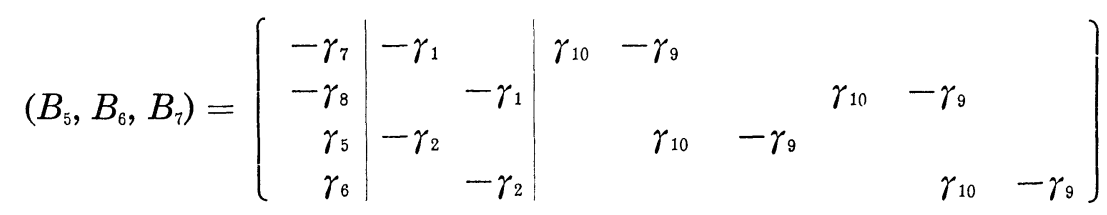


i) $V_{x_{16}}^{*}-S_{x_{16}}^{*} \leftrightarrow v_{8}+v_{9}+v_{11}+v_{15}+v_{16}=\left(u_{2} \wedge u_{5}+u_{3} \wedge u_{5}\right.$,

$$
\left.-u_{2} \wedge u_{4}-u_{3} \wedge u_{5}, u_{2} \wedge u_{3}+u_{1} \wedge u_{4}\right) \in S_{2,16}^{(2) *}
$$

ii) $\left(S_{x_{16}}^{*}\right)_{1} \leftrightarrow v_{9}+v_{10}+v_{11}+v_{16}=\left(u_{3} \wedge u_{5},-u_{2} \wedge u_{4}, u_{1} \wedge u_{4}+u_{1} \wedge u_{5}\right)$

$\in S_{3,15}^{(3) *} \leftrightarrow d \rho_{1}=-2 \varepsilon-\xi \leftrightarrow f_{1}^{*}(y)=y_{8}$

iii) $\left(S_{x_{16}}^{*}\right)_{2} \leftrightarrow v_{8}+v_{9}+v_{10}+v_{11}+v_{15}=\left(u_{2} \wedge u_{5},-u_{2} \wedge u_{4}-u_{3} \wedge u_{5}\right.$,

$\left.u_{2} \wedge u_{3}+u_{1} \wedge u_{4}+u_{1} \wedge u_{5}\right) \in S_{3,13}^{(2) *} \leftrightarrow d \rho_{2}=-8 \varepsilon-12 \eta$

$\leftrightarrow f_{2}^{*}(y)=\operatorname{det}\left(\begin{array}{ll}y_{11} & y_{14} \\ y_{13} & y_{16}\end{array}\right)^{2}-4 \operatorname{det}\left(\begin{array}{ll}y_{12} & y_{15} \\ y_{13} & y_{16}\end{array}\right) \cdot \operatorname{det}\left(\begin{array}{ll}y_{11} & y_{14} \\ y_{12} & y_{15}\end{array}\right)$

iv) $\left(S_{x_{16}}^{*}\right)_{3} \leftrightarrow v_{8}+v_{10}+v_{11}+v_{16}=\left(u_{3} \wedge u_{5},-u_{2} \wedge u_{4}, u_{1} \wedge u_{5}+u_{2} \wedge u_{3}\right)$

$\in S_{4,11}^{(3) *} \leftrightarrow d \rho_{3}=-4 \eta-2 \xi$

$\leftrightarrow f_{3}^{*}(y)=\operatorname{det}\left(\begin{array}{ll}y_{9} & y_{12} \\ y_{10} & y_{15}\end{array}\right)^{2}-\operatorname{det}\left(\begin{array}{ll}y_{9} & y_{11} \\ y_{10} & y_{14}\end{array}\right) \cdot \operatorname{det}\left(\begin{array}{ll}y_{9} & y_{13} \\ y_{10} & y_{16}\end{array}\right)$

v) $-\delta \chi=d \rho_{1}+d \rho_{2}+2 d \rho_{3}, \operatorname{tr}_{V_{x_{16}}^{*}}=2 d \rho_{1}+\frac{3}{2} d \rho_{2}+3 d \rho_{3}$.

(23) The isotropy subalgebra $g_{x_{18}}$ at $x_{18}$ is given as follows.

$$
\begin{aligned}
\mathfrak{g}_{x_{18}}= & \left\{A=\left(\frac{\varepsilon I_{4}+X}{0} \mid \frac{*}{-4 \varepsilon}\right) \oplus\left(\frac{-2 \varepsilon}{0} \mid \frac{*}{Y}\right) ; X \in \mathfrak{g} \mathfrak{p}(2), Y \in \mathfrak{g} \mathfrak{l}(2)\right\} \\
& \cong(\mathfrak{g} \mathfrak{l}(1) \oplus \mathfrak{g} \mathfrak{l}(1) \oplus \mathfrak{g} \mathfrak{F}(2) \oplus \mathfrak{g}(2)) \oplus V(6)
\end{aligned}
$$

The conormal vector space $V_{x_{18}}^{*}$ is spanned by $v_{1}=\left(0, u_{1} \wedge u_{3}-u_{2} \wedge u_{1}, 0\right)$, $v_{2}=\left(0, u_{1} \wedge u_{2}, 0\right), v_{3}=\left(0, u_{1} \wedge u_{4}, 0\right), v_{4}=\left(0, u_{3} \wedge u_{4}, 0\right), v_{5}=\left(0, u_{2} \wedge u_{3}, 0\right)$, $v_{6}=\left(0,0, u_{1} \wedge u_{3}-u_{2} \wedge u_{4}\right), v_{7}=\left(0,0, u_{1} \wedge u_{2}\right), v_{8}=\left(0,0, u_{1} \wedge u_{4}\right), v_{9}=(0$, $\left.0, u_{3} \wedge u_{4}\right), v_{10}=\left(0,0, u_{2} \wedge u_{3}\right), v_{11}=\left(0, u_{1} \wedge u_{5}, 0\right), v_{12}=\left(0, u_{2} \wedge u_{5}, 0\right), v_{13}=$ $\left(0, u_{3} \wedge u_{5}, 0\right), \quad v_{14}=\left(0, u_{4} \wedge u_{5}, 0\right), \quad v_{15}=\left(0,0, u_{1} \wedge u_{5}\right), v_{16}=\left(0,0, u_{2} \wedge u_{5}\right)$, $v_{17}=\left(0,0, u_{3} \wedge u_{5}\right), v_{18}=\left(0,0, u_{4} \wedge u_{5}\right)$. The action $d \rho_{x_{18}}$ of $g_{x_{18}}$ on $V_{x_{18}}^{*}$ is given by

$$
\begin{aligned}
& \left(v_{1}, \cdots, v_{18}\right) \\
& \mapsto\left(v_{1}, \cdots, v_{18}\right)\left(\begin{array}{c|c}
G L(1) \times G L(1) \times S p(2) \times S L(2) & 0 \\
2 \Lambda_{1}^{*} \otimes \Lambda_{1}^{*} \otimes \Lambda_{2} \otimes \Lambda_{1}^{*} & \\
\hline * & \begin{array}{c}
G L(1) \times G L(1) \times S p(2) \times S L(2) \\
3 \Lambda_{1} \otimes \Lambda_{1}^{*} \otimes \Lambda_{1} \otimes \Lambda_{1}^{*}
\end{array}
\end{array}\right)
\end{aligned}
$$

i) $V_{x_{18}}^{*}-S_{x_{18}}^{*} \leftrightarrow v_{2}+v_{4}+v_{8}+v_{10}+v_{15} \in S_{8,18}^{*}$

ii) $\left(S_{x_{18}}^{*}\right)_{1} \leftrightarrow v_{1}+v_{8}+v_{10}+v_{11} \in S_{8,18}^{*} \leftrightarrow d \rho_{1}=-2 \varepsilon-6 \eta \leftrightarrow \operatorname{deg} f_{1}^{*}=6$

iii) $\left(S_{x_{18}}^{*}\right)_{2} \leftrightarrow v_{1}+v_{7}+v_{17}+v_{18} \in S_{8,18}^{*} \leftrightarrow d \rho_{2}=-8 \varepsilon-4 \eta \leftrightarrow \operatorname{deg} f_{2}^{*}=4$

iv) $-\delta \chi=3 d \rho_{1}-2 d \rho_{2}, \operatorname{tr}_{V_{x_{1}}^{*}}=4 d \rho_{1}-\frac{3}{2} d \rho_{2}$.

(24) The isotropy subalgebra $g_{x_{21}}$ at $x_{21}$ is given as follows. 


$$
\begin{aligned}
\mathfrak{g}_{x_{21}}= & \left\{A=\left(\frac{3 \varepsilon I_{3}+X}{0} \mid \frac{*}{-2 \varepsilon I_{3}+Y}\right) \oplus\left(\frac{-6 \varepsilon}{0} \mid \frac{*}{\eta I_{2}+Z}\right)\right. \\
& \left.X, Z \in \mathfrak{g}(2), Y \in \mathfrak{I l}_{(3)}\right\} \\
& \cong\left(\mathfrak{g l}(1) \oplus \mathfrak{g l}(1) \oplus \mathfrak{g l}_{(2)} \oplus \mathfrak{g l}(2) \oplus \mathfrak{g l}(3)\right) \oplus V(8)
\end{aligned}
$$

Then $V_{x_{21}}^{*}$ is spanned by $v_{1}=\left(0, u_{4} \wedge u_{5}, 0\right), v_{2}=\left(0, u_{3} \wedge u_{5}, 0\right), v_{3}=\left(0, u_{3} \wedge\right.$ $\left.u_{4}, 0\right), v_{4}=\left(0,0, u_{4} \wedge u_{5}\right), v_{5}=\left(0,0, u_{3} \wedge u_{5}\right), v_{6}=\left(0,0, u_{3} \wedge u_{4}\right), v_{7}=\left(u_{4} \wedge\right.$ $\left.u_{5}, 0,0\right), v_{8}=\left(u_{3} \wedge u_{5}, 0,0\right), v_{9}=\left(u_{3} \wedge u_{4}, 0,0\right), v_{10}=\left(0, u_{1} \wedge u_{3}, 0\right), v_{11}=(0$, $\left.u_{1} \wedge u_{4}, 0\right), v_{12}=\left(0, u_{1} \wedge u_{5}, 0\right), v_{13}=\left(0, u_{2} \wedge u_{3}, 0\right), v_{14}=\left(0, u_{2} \wedge u_{4}, 0\right), v_{15}=$ $\left(0, u_{2} \wedge u_{5}, 0\right), \quad v_{16}=\left(0,0, u_{1} \wedge u_{3}\right), \quad v_{17}=\left(0,0, u_{1} \wedge u_{4}\right), \quad v_{18}=\left(0,0, u_{1} \wedge u_{5}\right)$, $v_{19}=\left(0,0, u_{2} \wedge u_{3}\right), v_{20}=\left(0,0, u_{2} \wedge u_{4}\right), v_{21}=\left(0,0, u_{2} \wedge u_{5}\right)$. The action $d \rho_{x_{21}}$ of $\mathfrak{g}_{x_{21}}$ on $V_{x_{21}}^{*}$ is given by $\left(v_{1}, \cdots, v_{21}\right)$

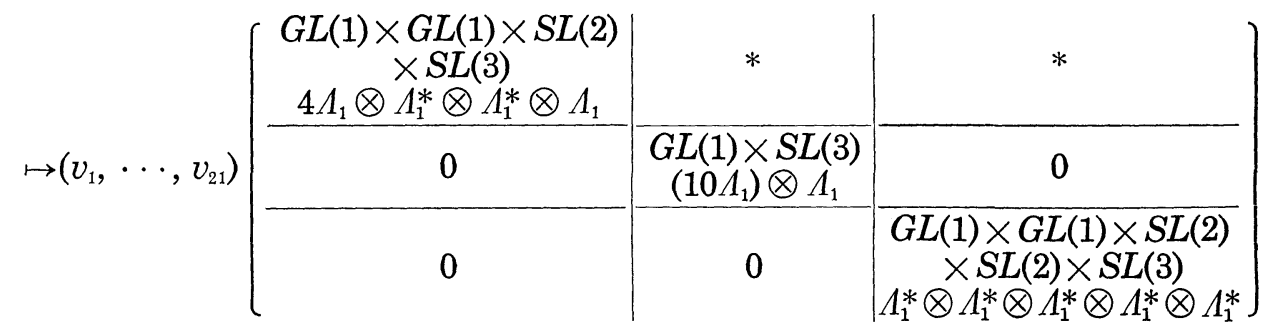

i) $V_{x_{21}}^{*}-S_{x_{21}}^{*} \leftrightarrow v_{7}+v_{10}+v_{14}+v_{18}+v_{19}=\left(u_{4} \wedge u_{5}, u_{1} \wedge u_{3}+u_{2} \wedge u_{4}\right.$, $\left.u_{1} \wedge u_{5}+u_{2} \wedge u_{3}\right) \in S_{1,21}^{(2) *}$

ii) $\left(S_{x_{21}}^{*}\right)_{1} \leftrightarrow v_{8}+v_{10}+v_{14}+v_{18}+v_{19}=\left(u_{3} \wedge u_{5}, u_{1} \wedge u_{3}+u_{2} \wedge u_{4}\right.$, $\left.u_{1} \wedge u_{5}+u_{2} \wedge u_{3}\right) \in S_{3,13}^{(2)} \leftrightarrow d \rho_{1}=18 \varepsilon-2 \eta \leftrightarrow \operatorname{deg} f_{1}^{*}=4$

iii) $\left(S_{x_{21}}^{*}\right)_{2} \leftrightarrow v_{8}+v_{9}+v_{10}+v_{14}+v_{18}=\left(u_{3} \wedge u_{4}+u_{3} \wedge u_{5}, u_{1} \wedge u_{3}\right.$ $\left.+u_{2} \wedge u_{4}, u_{1} \wedge u_{5}\right) \in S_{2,16}^{(2) *} \leftrightarrow d \rho_{2}=-6 \varepsilon-6 \eta \leftrightarrow \operatorname{deg} f_{2}^{*}=6$

iv) $-\delta \chi=2 d \rho_{1}+d \rho_{2}, \operatorname{tr}_{V_{x_{21}}^{*}}=3 d \rho_{1}+2 d \rho_{2}$.

(25) The isotropy subalgebra $g_{x_{30}}$ at $x_{30}=0$ is $\mathrm{g}$ itself.

This is a good holonomic variety and $\operatorname{ord}_{A} f^{s}=-15 s-\frac{30}{2}$. Thus we obtain the holonomy diagram (Figure 11-1). From this diagram, we obtain the $b$-function $b(s)=\left((s+1)\left(s+\frac{3}{2}\right)(s+2)\right)^{3} \cdot\left(\left(s+\frac{4}{3}\right)\left(s+\frac{5}{3}\right)\right)^{2} \cdot\left(s+\frac{5}{4}\right)\left(s+\frac{7}{4}\right)$.

Remark. Let $\Lambda_{0}=\overline{G\left(x_{0}, y_{0}\right)}$ and $\Lambda_{1}=\overline{G\left(x_{1}, y_{1}\right)}$ be good holonomic varieties satisfying $\left(x_{0}, y_{1}\right) \in \Lambda_{0} \cap \Lambda_{1}$ and $\operatorname{dim} G\left(x_{0}, y_{1}\right)=\operatorname{dim} V-1$. Then we can calculate $\beta$ by Proposition 1-4. It is known that if $\beta$ depends on the choice of $A_{1}$, then $\left(x_{0}, y_{1}\right)$ is not contained in other $\Lambda_{i}(i \neq 0,1)$, i.e., there are no three $\Lambda_{i}$ 's which intersect at $\left(x_{0}, y_{1}\right)$ with codimension one. (If more 


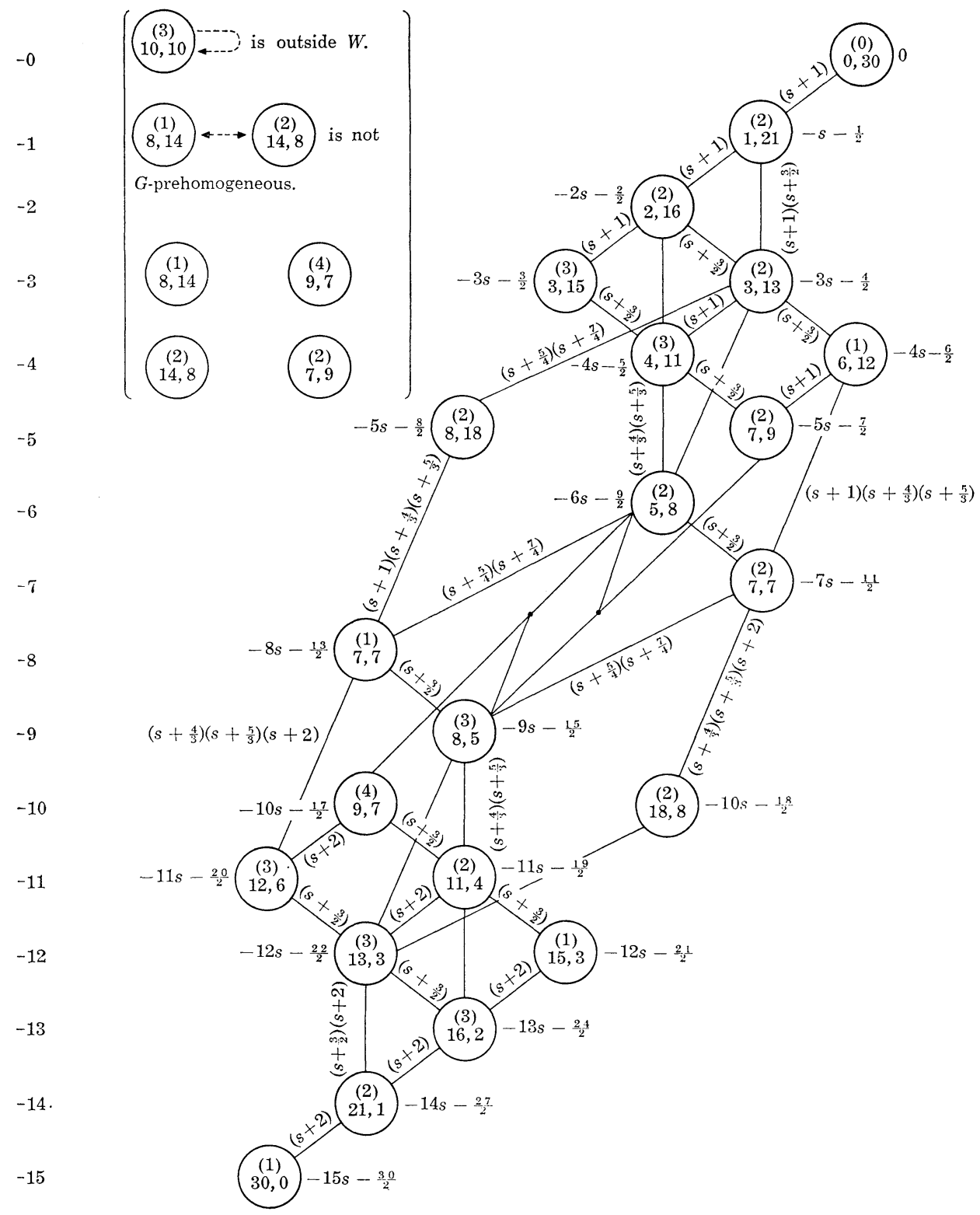

Fig. 11-1. Holonomy diagram of $\left(S L(5) \times G L(3), \Lambda_{2} \otimes \Lambda_{1}, V(10) \otimes V(3)\right)$ where $\left(\begin{array}{c}(1) \\ i j\end{array}\right.$ denotes the conormal bundles of the orbit $S_{i j}^{(k)}$ in Proposition 6-1. 
than two $\Lambda_{i}$ 's intersect with codimension one, then $\beta=1$ and it does not depend on $A_{1}$.) All one-codimensional intersections obtained from (1) (14) satisfy this condition except $\Lambda_{5,8}^{(2)} \cap \Lambda_{4,11}^{(3)}$ and $\Lambda_{8,5}^{(3)} \cap \Lambda_{7,9}^{(2)}$. In general, if $\left(x_{0}, y_{1}\right)$ $\in \overline{G\left(x_{2}, y_{2}\right)}$, then we have $\operatorname{codim}_{V} \rho(G) x_{0} \geqq \operatorname{codim}_{V} \rho(G) x_{1}$ and $\operatorname{codim}_{V^{*}}{ }^{*}(G) y_{1}$ $\geqq \operatorname{codim}_{V^{*}} \rho^{*}(G) y_{2}$. From this, there are no other $\Lambda^{\prime}$ 's satisfying $\operatorname{dim} \Lambda \cap \Lambda_{\delta, 8}^{(2)} \cap$ $\Lambda_{4,11}^{(3)}=\operatorname{dim} V-1$. For $\Lambda_{8,5}^{(3)} \cap \Lambda_{7,9}^{(2)}$, it is enough to check $\Lambda_{7,7}^{(1)}, \Lambda_{7,7}^{(2)}$ and $\Lambda_{5,8}^{(2)}$. By using the duality, i.e., $(G, \rho, V) \cong\left(G, \rho^{*}, V^{*}\right)$, we get all one-codimensional intersections of three good holonomic varieties.

\section{\$12. Table of the $b$-functions of irreducible reduced regular P.V.'s}

(1) $\left(G \times G L(m), \rho \otimes \Lambda_{1}, V(m) \otimes V(m)\right)$ where $\rho: G \rightarrow G L(V(m))$ is an $m$ dimensional irreducible representation of a connected semi-simple algebraic group $G$ (or $G=\{1\}$ and $m=1$ ).

$b(s)=(s+1)(s+2) \cdots(s+m)$ (See Figure 2-1 and 2-4).

(2) $\left(G L(n), 2 \Lambda_{1}, V\left(\frac{1}{2} n(n+1)\right)\right)(n \geqq 2)$

$b(s)=\prod_{\nu=1}^{n}\left(s+\frac{\nu+1}{2}\right)=(s+1)\left(s+\frac{3}{2}\right) \cdots\left(s+\frac{n+1}{2}\right)$

(See Figure 2-2 and 2-4).

(3) $\left(G L(2 m), \Lambda_{2}, V(m(2 m-1))\right)(m \geqq 3)$

$b(s)=\prod_{k=1}^{m}(s+2 k-1)=(s+1)(s+3) \cdots(s+2 m-1)$

(See Figure 2-3 and 2-4).

(4) $\left(G L(2), 3 \Lambda_{1}, V(4)\right)$

$b(s)=(s+1)^{2}\left(s+\frac{5}{6}\right)\left(s+\frac{7}{6}\right)($ See $[2])$.

(5) $\left(G L(6), \Lambda_{3}, V(20)\right)$

$b(s)=(s+1)\left(s+\frac{5}{2}\right)\left(s+\frac{7}{2}\right)(s+5)$ (See Figure 8-1).

(6) $\left(G L(7), \Lambda_{3}, V(35)\right)$

$b(s)=(s+1)(s+2)\left(s+\frac{5}{2}\right)\left(s+\frac{7}{2}\right)(s+3)(s+4)(s+5)$

(See Figure 10-1).

( 7) $\left(G L(8), \Lambda_{3}, V(56)\right)$

$b(s)=(s+1)\left(s+\frac{3}{2}\right)^{2}\left(s+\frac{11}{6}\right)(s+2)^{3}\left(s+\frac{13}{6}\right)\left(s+\frac{7}{3}\right)\left(s+\frac{5}{2}\right)^{3}\left(s+\frac{8}{5}\right)(s+3)^{2}\left(s+\frac{7}{2}\right)$

(See [10]).

( 8) $\quad\left(S L(3) \times G L(2), 2 \Lambda_{1} \otimes \Lambda_{1}, V(6) \otimes V(2)\right)$

$b(s)=\left\{(s+1)^{2}\left(s+\frac{5}{6}\right)\left(s+\frac{7}{6}\right)\left(s+\frac{3}{4}\right)\left(s+\frac{5}{4}\right)\right\}^{2}$ (See [12]).

( 9$) \quad\left(S L(6) \times G L(2), \Lambda_{2} \otimes \Lambda_{1}, V(15) \otimes V(2)\right)$

$b(s)=(s+1)^{2}\left(s+\frac{5}{6}\right)\left(s+\frac{7}{6}\right)\left(s+\frac{3}{2}\right)^{2}(s+2)^{2}\left(s+\frac{5}{2}\right)^{2}\left(s+\frac{7}{3}\right)\left(s+\frac{8}{3}\right)$

(See [12]). 
(10) $\quad\left(S L(5) \times G L(3), \Lambda_{2} \otimes \Lambda_{1}, V(10) \otimes V(3)\right)$

$b(s)=\left((s+1)\left(s+\frac{3}{2}\right)(s+2)\right)^{3} \cdot\left(\left(s+\frac{4}{3}\right)\left(s+\frac{5}{3}\right)\right)^{2} \cdot\left(s+\frac{5}{4}\right)\left(s+\frac{7}{4}\right)$

(See Figure 11-1).

(11) $\quad\left(S L(5) \times G L(4), \Lambda_{2} \otimes A_{1}, V(10) \otimes V(4)\right)($ See [11]).

(12) $\quad\left(S L(3) \times S L(3) \times G L(2), \Lambda_{1} \otimes \Lambda_{1} \otimes \Lambda_{1}, V(3) \otimes V(3) \otimes V(2)\right)$

$b(s)=(s+1)^{4}\left(s+\frac{3}{2}\right)^{4}\left(s+\frac{4}{3}\right)\left(s+\frac{5}{3}\right)\left(s+\frac{5}{6}\right)\left(s+\frac{7}{6}\right)($ See [12]).

(13) $\quad\left(S p(n) \times G L(2 m), \Lambda_{1} \otimes \Lambda_{1}, V(2 n) \otimes V(2 m)\right)(n \geqq 2 m \geqq 2)$

$$
\begin{aligned}
b(s)= & \prod_{k=1}^{m}(s+2 k-1) \prod_{\ell=0}^{m-1}(s+2 n-2 \ell) \\
= & (s+1)(s+3) \cdots(s+2 m-1)(s+2 n)(s+2 n-2) \cdots \\
& (s+2 n-2 m+2) \text { (See Figure } 3-1 \text { and } 3-2) .
\end{aligned}
$$

(14) $\quad\left(G L(1) \times S p(3), \square \otimes \Lambda_{3}, V(1) \otimes V(14)\right)$

$b(s)=(s+1)(s+2)\left(s+\frac{5}{2}\right)\left(s+\frac{7}{2}\right)$ (See Figure 9-1).

(15)

$$
\begin{aligned}
&\left(S O(n) \times G L(m), \Lambda_{1} \otimes \Lambda_{1}, V(n) \otimes V(m)\right) \quad\left(n \geq 3, \frac{n}{2} \geqq m \geqq 1\right) \\
& b(s)= \prod_{k=1}^{m}\left(s+\frac{k+1}{2}\right) \prod_{\ell=1}^{m}\left(s+\frac{n-\ell+1}{2}\right) \\
&=(s+1)\left(s+\frac{3}{2}\right) \cdots\left(s+\frac{m+1}{2}\right)\left(s+\frac{n}{2}\right)\left(s+\frac{n-1}{2}\right) \cdots \\
&\left(s+\frac{n-m+1}{2}\right) \quad \text { (See [2]). }
\end{aligned}
$$

(16) $\quad(G L(1) \times \operatorname{Spin}(7), \square \otimes$ spin rep., $V(1) \otimes V(8))$

$b(s)=(s+1)(s+4) \quad($ See Remark in $\S 5)$.

(17) $\quad \operatorname{Spin}(7) \times G L(2)$, spin rep. $\left.\otimes \Lambda_{1}, V(8) \otimes V(2)\right)$

$b(s)=(s+1)\left(s+\frac{3}{2}\right)(s+4)\left(s+\frac{7}{2}\right) \quad$ (See Remark in $\left.\S 5\right)$.

(18) $\quad\left(\operatorname{Spin}(7) \times G L(3)\right.$, spin rep. $\left.\otimes \Lambda_{1}, V(8) \otimes V(3)\right)$

$b(s)=(s+1)\left(s+\frac{3}{2}\right)(s+2)(s+4)\left(s+\frac{7}{2}\right)(s+3)$ (See Remark in $\left.\S 5\right)$.

(19) $\quad(G L(1) \times \operatorname{Spin}(9), \square \otimes$ spin rep., $V(1) \otimes V(16))$

$b(s)=(s+1)(s+8) \quad($ See Remark in $\S 5)$.

(20) $\quad\left(\right.$ Spin $(10) \times G L(2)$, half-spin rep. $\left.\otimes \Lambda_{1}, V(16) \otimes V(2)\right)$

$b(s)=(s+1)(s+4)(s+5)(s+8) \quad$ (See Figure 4-1).

(21) $\quad\left(\right.$ Spin $(10) \times G L(3)$, half-spin rep. $\left.\otimes A_{1}, V(16) \otimes V(3)\right)$

$b(s)=(s+1)\left(s+\frac{3}{2}\right)(s+2)(s+3)\left(s+\frac{7}{2}\right)(s+4)\left(s+\frac{5}{3}\right)\left(s+\frac{6}{3}\right)\left(s+\frac{7}{3}\right) \times$ $\times\left(s+\frac{8}{3}\right)\left(s+\frac{9}{3}\right)\left(s+\frac{10}{3}\right) \quad$ (See [15]).

(22) $\quad(G L(1) \times \operatorname{Spin}(11), \square \otimes$ spin rep., $V(1) \otimes V(32))$

$b(s)=(s+1)\left(s+\frac{7}{2}\right)\left(s+\frac{11}{2}\right)(s+8) \quad$ (See Remark in $\left.\S 5\right)$.

(23) $\quad(G L(1) \times \operatorname{Spin}(12), \square \otimes$ half-spin rep., $V(1) \otimes V(32))$

$b(s)=(s+1)\left(s+\frac{7}{2}\right)\left(s+\frac{11}{2}\right)(s+8) \quad$ (See Figure 5-1). 
(24) $\quad(G L(1) \times$ Spin (14), $\square \otimes$ half-spin rep., $V(1) \otimes V(64))$

$b(s)=(s+1)\left(s+\frac{5}{2}\right)\left(s+\frac{7}{2}\right)(s+4)(s+5)\left(s+\frac{11}{2}\right)\left(s+\frac{13}{2}\right)(s+8)$

(See Appendix).

(25) $\quad\left(G L(1) \times\left(G_{2}\right), \square \otimes A_{2}, V(1) \otimes V(7)\right)$

$b(s)=(s+1)\left(s+\frac{7}{2}\right) \quad($ See Remark in $\S 5)$.

(26) $\quad\left(\left(G_{2}\right) \times G L(2), \Lambda_{2} \otimes \Lambda_{1}, V(7) \otimes V(2)\right)$

$b(s)=(s+1)\left(s+\frac{3}{2}\right)\left(s+\frac{7}{2}\right)(s+3) \quad$ (See Remark in $\left.\S 5\right)$.

(27) $\quad\left(G L(1) \times E_{6}, \square \otimes \Lambda_{1}, V(1) \otimes V(27)\right)$

$b(s)=(s+1)(s+5)(s+9) \quad$ (See Figure 6-1).

(28) $\quad\left(E_{6} \times G L(2), \Lambda_{1} \otimes \Lambda_{1}, V(27) \otimes V(2)\right)$

$b(s)=(s+1)^{2}\left(s+\frac{5}{6}\right)\left(s+{ }_{6}^{7}\right)\left(s+\frac{5}{2}\right)^{2}(s+3)^{2}\left(s+\frac{9}{2}\right)^{2}\left(s+\frac{13}{3}\right)\left(s+\frac{14}{3}\right)$

(See [12]).

(29) $\quad\left(G L(1) \times E_{7}, \square \otimes \Lambda_{6}, V(1) \otimes V(56)\right)$

$b(s)=(s+1)\left(s+\frac{11}{2}\right)\left(s+\frac{19}{2}\right)(s+14) \quad$ (See Figure 7-1).

We can obtain the $b$-functions of all irreducible regular P.V.'s, except for those in the castling class of (11), from the Table above and the following theorem due to T. Shintani.

Theorem (T. Shintani). Let $\left(G^{\prime}, \rho^{\prime}, V^{\prime}\right)$ be a castling transform of an irreducible regular P.V. $(G, \rho, V)$, i.e., there exists a triplet $(\tilde{G}, \tilde{\rho}, V(m))$ and a positive number $n$ with $m>n \geqq 1$ such that

$$
\begin{aligned}
& (G, \rho, V) \cong\left(\tilde{G} \times G L(n), \tilde{\rho} \otimes \Lambda_{1}, V(m) \otimes V(n)\right) \\
& \left(G^{\prime}, \rho^{\prime}, V^{\prime}\right) \cong\left(\tilde{G} \times G L(m-n), \tilde{\rho}^{*} \otimes \Lambda_{1}, V(m)^{*} \otimes V(m-n)\right) .
\end{aligned}
$$

Then the b-functions $b(s)$ and $b^{\prime}(s)$ of them satisfy

$$
\begin{aligned}
& b(s) \prod_{i=1}^{d}(d s-i)(d s-i+1) \cdots(d s-i+m-n-1) \\
& \quad=b^{\prime}(s) \prod_{i=1}^{d}(d s-i)(d s-i+1) \cdots(d s-i+n-1)
\end{aligned}
$$

where $\operatorname{deg} f=d m$ and $\operatorname{deg} f^{\prime}=d(m-n)$. Here $f$ and $f^{\prime}$ are the basic relative invariants of $(G, \rho, V)$ and $\left(G^{\prime}, \rho^{\prime}, V^{\prime}\right)$ respectively.

\section{Appendix with I. Ozeki}

Here we consider the regular irreducible P.V. $(G L(1) \times \operatorname{Spin}(14)$, $\square \otimes$ half-spin rep., $V(1) \otimes V(64))$. The orbital decomposition of this space has been done by the author and I. Ozeki ([7]), by Popov ([9]), by V. Gatti and E. Viniberghi ([10]). There exist ten orbits, and the conormal bundle 
of each orbit is a good Lagrangian variety. The relative invariant of this space is of degree eight ([1]), and its $b$-function is given by $b(s)=(s+1)$ $\left(s+\frac{5}{2}\right)\left(s+\frac{7}{2}\right)(s+4)(s+5)\left(s+\frac{11}{2}\right)\left(s+\frac{13}{2}\right)(s+8)$. Its holonomy diagram is given by Figure A, where we denote by $m$ the conormal bundle $A$ of the $m$-codimensional orbit.

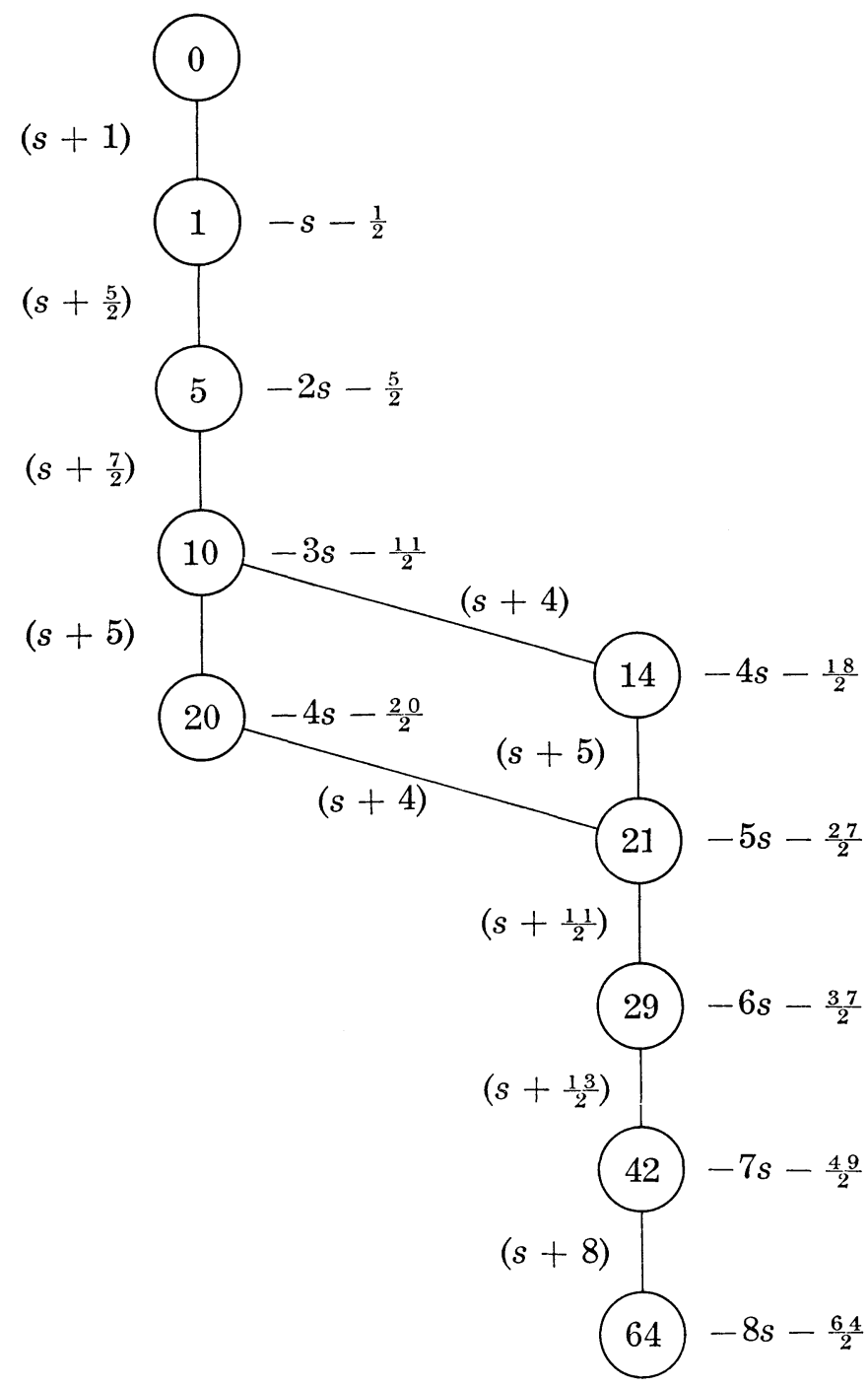

Figure A. Holonomy“-diagram of $(G L(1) \times \operatorname{Spin}(14)$, $\square \otimes$ half-spin rep., $V(1) \otimes V(64))$ 


\section{REFERENCES}

[1] M. Sato and T. Kimura, A classification of irreducible prehomogeneous vector spaces and their relative invariants, Nagoya Math. J., 65 (1977), 1-155.

[2] M. Sato, M. Kashiwara, T. Kimura and T. Oshima, Micro-local analysis of prehomogeneous vector spaces, Inv. math., 62 (1980), 117-179.

[ 3 ] J-I. Igusa, A classification of spinors up to dimension twelve, Amer. J. of Math., 92, No. 4 (1970), 997-1028.

[4] C. Chevalley, The Algebraic Theory of Spinors, Columbia University Press, 1954.

[5] S. J. Haris, Some irreducible representation of exceptional algebraic groups, Amer. J. of Math., 93 (1971).

[6] G. B. Gurevich, Foundations of the Theory of Algebraic Invariants, P. Noordhoff LTD-Groningen, 1964.

[7] T. Kimura, Study of irreducible prehomogeneous vector spaces, Master Thesis in Japanese, University of Tokyo, 1973, $373 \mathrm{pp}$.

[8] H. Kawahara, Prehomogeneous vector spaces related with the spin group, Master Thesis in Japanese, University of Tokyo, 1974.

[9] V. L. Popov, A classification of spinors of dimension fourteen, Uspechi Math. Nauk, 32, (1977).

[10] V. Gatti and E. Viniberghi, Spinors of 13-dimensional space, Adv. Math., 30, No. 2 (1978).

[11] I. Ozeki, On the micro-local structure of the regular prehomogeneous vector space associated with $S L(5) \times G L(4)$, I, Proc. Japan Acad., 55, Ser. A, (1979).

[12] T. Kimura and M. Muro, On some series of regular irreducible prehomogeneous vector spaces, Proc. Japan Acad., 55, Ser A, No. 10 (1979), 384-389.

[13] I. Ozeki, On the micro-local structure of a regular prehomogeneous vector space associated with $G L(8)$, Proc. Japan Acad., 56, Ser. A, No. 1 (1980), 18-21.

[14] T. Kimura, Remark on some combinatorial construction of relative invariants, Tsukuba J. of Math., 5, No.1 (1981).

[15] T. Kimura and I. Ozeki, On the micro-local structure of regular prehomogenous vector space associated with Spin $(10) \times G L(3)$. (in preparation).

Institute of Mathematics

University of Tsukuba 